$S L A C-294$

(E)

\section{MEASUREMENTS OF CHARGED TWO-PARTICLE EXCLUSIVE STATES IN PHOTON-PHOTON INTERACTIONS *}

Robert Prestan Johnson

Stanford Linear Accelerator Center Stanford University

Stanford, California 94305

March 1986

Prepared for the Department of Energy under contract number DE-AC03-76SF00515

Printed in the United States of America. Available from the National Technical Information Service, U.S. Department of Commerce, 5285 Port Royal Road, Springfield, Virginia 22161. Price: Printed Copy A 12, Microfiche A01.

* Ph.D. Dissertation
A description is given of an experiment performed at the PEP electronpositron storage ring, using the DELCO detector, to measure the formation of charged particle pairs from interactions of pairs of virtual photons radiated from the colliding electron beafns. The final states which are measured are electronpositron pairs, charged pion pairs, charged kaon puirs, and proton pairs.

Electron-positron pairs are separated from other data by use of gas Cerenkoy counters. The shapes of all kinematic distributions are found to agree with predictions of quantum electrodynamics. These data also are used as an accurate normalization for subtraction of the muon-pair background and for measurement of the cross sections of the three hadronic channels.

Pion pairs are measured in the mass range from 0.6 to $2.0 \mathrm{GeV}$, where production of the $f(1270)$ resonance is observed to interfere with significant continuum production. The continuum is well described by single-pion exchange, allowing a measurement of the $f$ two-photon partial width of $3.47 \pm 0.37 \mathrm{keV}$. No a priori assumption is made about the ratio of helicity amplitudes, and the phenomenological model used in fitting the data is constrained to satisfy elastic unitarity. If unitarity is not required, then the isted partial width is a factor of 0.83 lower than the quoted value. The $Q^{2}$ dependence of the cross section is found to be consistent with predictions of the Generalized Vector Dominance Model.

Kaon pairs and proton pairs are identified by time-of-fight measurements. Kaon pairs are measured in the mass range from 1.3 to $2.0 \mathrm{GeV}$, where production of the $f^{\prime}(1520)$ resonance is observed. The continuum background is estimated by extrapolation, allowing a men ...ent of the $f^{\prime}$ two-photon partial width of $0.07 \pm 0.04 \mathrm{keV}$. The it.uo of the $f$ and $f^{\prime}$ two-photon partial widths is found to 1 4t:lstent with $S U(3)$ quark model predictions with a mixing angle of $28 \pm 4$ degrees. Twenty-three proton pairs are observed, and the average cross section for their production from photon-photon collisions in the mass range from 2.2 to $2.9 \mathrm{GeV}$ is measured over the angular range $-0.6<\cos \theta<0.6$ to be $1.2 \pm 0.5 \mathrm{nb}$. 


\section{Acknowledgement}

The experiment described in this thesis has been made possible by the combined efforts of many people. The DELCO collaboration consists of 38 physicists in addition to myself: W.I. Atwood, P.H. Baillon, B.C. Barish, G.R. Bonneaud, A. Courau, H. DeStaebler, G.J. Donaldson, R. Dubois, M.M. Duro, E.E. Elsen, S.G. Gao, Y.Z. Huang, G.M. Irwin, H. Kichimi, J. Kirkby, D.E. Klem, D.E. Koop, J Ludwig, G.B. Mills, A. Ogawa, T. Pal, D. Peret-Galix, R. Pitthan, D.L. Pollard, C.Y. Prescott, L.Z. Rivkin, L.S. Rochester, W. Ruckstuhl, M. Sakuda, S.S. Sherman, E.J. Siskind, R. Stroynowski, R.E. Taylor, S.Q. Wang, S.G. Wojcicki, H. Yamamoto, W.G. Yan, and C.C. Young. I thank all of these individuals for their efforts and devotion to the experiment, for helping me with many problems that 1 have encountered, and in general for the pleasant experience I have had working with them at SLAC. I would like to give special thanks to Bill Atwood for his unfailing en thusiasm for the experiment and associated physics analyses and for the special efforts he has made to assist me; to Hobje DeStaebler for mary helpfu] discussions at points of impasse in my work; and to Eckhard Elsen for all the help and encouragement he has given me and for a very special friendship. I am especially grateful to my thesis advisor, Prof. Charles Prescott, for steering me toward this thesis topic, for his encouragement and guidance during difficult times, and for seeing my graduate work through to completion.

I would like to acknowledge the support for DELCO by the technical staffs of SLAC, groups A and G, and CalTech: B. Bricaud, A. Johnson, O. Saxton, S. Sund, J. Zingheim, D. Chambers, D. Ouimette, C. Pierce, D. Porat, M Susskind, A. Tillman, R. Cooper, L. Cronk, R. Decker, H. Grau, J. Hanson, L. Mossbarger, D. Sell, S. Sondergaard, and J. Ungerer. Without their work, this experiment would not have been possible. 1 am equally indebted to the PEP Storage Ring Division for providing the hundreds of hours of stable colliding beams necessary for successful data taking.

The DELCO experiment is supported through CalTech and SLAC by the
U.S. Department of Energy and the National Science Foundation. The data have been analyzed with the IBM computers of SLAC Computing Services. Most of the figures were made with the program Top Drawer by R.B. Cnaffee, and the typesetting was accomplished with the TEX typesetting program of Donald $\mathrm{E}$. Knuth, using the $\left.P_{s} i_{2}\right]$ macros of Arthur Ogawa for the format.

This work would not have been possible without the initiative and experience of André Courau, who as a visitor from Laboratoire de l'Accelerateur Lineaire of France launched the DELCO analysis of two-photon channels and guided me in my work up through the time of publication of our first results. I also give special thanks to Gerard Bonneaud and Steve Sherman, who contributed directly to the analysis of two-photon channels and assisted me in many ways, to Prof. Frederick Gilman for several useful discussions about theoretical aspects of two photon interactions, and to René Ong for helping to proofread the manuscript.

Finally, I would like to thank all of my friends who have made my time at Stanford a most pleasant experience. I especially thank my parents for their continual support and encouragement and my wife, Mayumi, and son, Kent, for the love and joy that they have given to me during the course of this work.

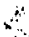

\section{DISCLAIMER}

of work sponsored by an agency of the United States This report was prepared as an account of work sponoter by any of their Government. Neither the United States Government nor any ageney leal liability or responsiGover makes any wartunty, express or implied, or assumes any lrgal homilys product or employecs, makes any completeness, of usefulness of any information, apparatus, product or bility for the accuracy, completeness, or use would not infringe privalely owned rights. Referprocess disclosed, or represents that its use would not in, or service by trade name, trademark. ence herein to any specilic commercial product, process, or sectite or imply its endorsement, recommanufacturer, or otherwise does not necessarily constitute or imply ils en thereof. The views mendation, or favoring by the United States Governmensarily state or reflect those of the

and opinions of authors expressed herein do 
Table of Contents

Abstract + . . . . . . . . . . . . . . .

Acknowledgernents . . . . . . . . . . . . iii

Table of Contents . . . . . . . . . . . . . . v

List of Tables . . . . . . . . . . . . . . . ix

List of Figures . . . . . . . . . . . . . $\mathrm{x}$

1. The Two-Photon Particle Production Process . . . . . I

1.1 Introduction . . . . . . . . . . . . 1

1.2 Hadron Production from Photon-Photon Interactions . . . 4

1.3 Resonance Formation . . . . . . . . . . 6

1.4 Angular Distributions of Tensor Meson Decays . . . . 8

1.5 Unitary Symmetry and the Tensor Meson Nonet - . . . 11

2. Two-Photon Interactions at $e^{+} e^{-}$Storage Ringa . . . . . 17

2.1 Kinematics . . . . . . . . . . . , 17

2.2 Cross Sections . . . . . . . , . . . . 20

2.3 The Equivalent Photon Approximation . . . . . . 24

2.4 Luminosity Function for Tagged Events . . . . . . 25

2.5 Extrapolations of Cross Sections to Large $Q^{2}$. . . . . . 28

3. Description of the Apparatus . . . . . . . . . 30

3.1 Tracking System . . . . . . . . . . . 30

3.2 Cerenkov Counter System . . . . . . . . . 39

3.3 Barrel Shower Counters . . . . . . . . . 40

3.4 Poletip Shower Counters . . . . . . . . . 43

3.5 Tirne of Flight Counters . . . . . . . . . 44

3.6 Luminosity Monitor . . . . . . - . . , 44

3.7 Event Trigger . . . . . . . . . . . . 45

3.8 Data Processing and Reduction . . . . . . . . . 46

3.8.1 Pass-1 Filter . . . . . . . . . . . . 46

3.8.2 Event Classification . . . . . . . . . 48
3.8.3 Track Fitting . . . . . . . . . . . 49

4. The QED Channels . . . . . . . . . . . 51

4.1 Complete Calculations of the Cross Sections . . . . . 51

4.2 The DELCO Experimental Acceptance for $e^{+} e^{-} \rightarrow e^{+} e^{-} e^{+} e^{-} \quad$. 53

4.3 Separation of Electrons from More Massive Particles - . . 54

4.4 Electron Identification Efficiency . . . . . . . . 58

4.5 Trigger Efficiency for Electron Pairs , . . . . . . 61

4.6 Summary of Cuts Made on Untagged Electron Pairs . . . 69

4.7 The Mronte Carlo Simulation . . . . . . . . 72

4.8 Unfolding Detector Effects - . . . . . . . . 74

4.9 Measurements of Tagged $e^{+} e^{-} \rightarrow e^{+} e^{-} e^{+} e^{-}$Events , . . 79

4.9.1 Analysis of the Luminosity-Counter Energy . . . 80

4.9.2 Measured Kinernatic Distributions . . . . . 82

4.10 Measurement of the Integrated Luminosity . . . . 85

4.11 Integrated Luminosity from Tagged Events . . . . . . 93

5. Measuring the Pion-Pair Spectrum . . . . . 95

5.1 Rejection of Four-Electron Events + . . . . . . . 97

5.2 Analysis Cuts for the Untagged $\pi^{+} \pi^{-}$Signal . . . . . . 99

5.3 Subtraction of the $\mu^{+} \mu^{-}$Background . . . . . . 105

5.4 Consideration of Other Backgrounds to $\gamma \gamma \rightarrow \pi^{+} \pi^{-}$. . . 113

5.5 The Tagged Pion-Pair Spectrum . . . . . . . . 118

6. Mcasurement of the $\pi^{+} \pi^{-}$Trigger Efficiency , . , , , , 124

6.1 Shower Counter Latch Efficiency from Tagged Data . . . 124

6.2 Cajculation of the Shower Counter Latch Efficiency . . . 129

6.3 Trigger Efficiency for Pion Pairs . . . . . . . . . 131

7. Theoretical Models for $\gamma \gamma \rightarrow \pi^{+} \pi^{-}$. . . . . . . . . 133

7.1 Description of the Incoming Two-Photon State . . . . 135

7.2 The Scattering Cross Section . . . . . . . . 138

7.3 Description of $\pi \pi$ Elastic Scattering . . . . . . . . . 140 
7.4 Unitarity, Analyticity and the Model for $\gamma \gamma \rightarrow \pi^{+} \pi^{-} \quad$. . . 141

i.4.1 The Born Term and Fixed-t Dispersion Relations . . . 142

7.4.2 Resonant Partial Waves . . . . . . . . . 145

7.4.3 Estimate of Contributions from the Left Hand Cut . . . 148

7.4.4 Corrections Required by Unitarity . . . . . . 150

7.4.5 Justification of the Born Approximation . . . . . 152

7.4.6 Constraints on the Coupling of $\gamma \gamma$ to the $f$ Resonance . . 153

7.4.7 Further Consideration of the Unitarity Condition . . . 155

7.5 The Model of Mennessier . . . . . . . . 157

7.6 Comparison of the Two Models . . . . . . . . . . 159

8. Fitting the $\pi^{+} \pi^{-}$Spectra , . , , . , , , , 168

8.1 Summary of the $\gamma \gamma \rightarrow \pi^{+} \pi^{-}$Model . . . . . . . 168

8.2 The Fitting Method . . . . . . . . . . . 169

8.3 Fit Results for the Untagged Analysis _. . . . . . . 173

8.3.1 Fit to the $\pi^{+} \pi^{-}$Model Without Unitarization . . . . 173

8.3.2 Effect of Requiring Unitarity . . . . . . . 179

8.3.3 Comparison with the Mennessier Model . . . . . 180

8.3.4 Including the Angular Distribution . . . . . . 182

8. 3.5 Extrapolating $\Gamma_{\gamma \gamma}$ to $Q^{2}=0 \quad$, . . . . . . 186

8.4 Fit Results for the Tagged Analysis . . . . . . . . 186

8.5 Remarks on the Fitted $f$ Mass . . . . . , , , , 190

9. The Time-of-Flight Analysis . . . . . . . . . 191

9.1 Calibration of the Time-of-Flight System . . . . . . . 192

9.2 Monte Carlo Simulation . . . . . . . . . 193

9.3 Time Consistency and the Timing Resolution . . . . . 196

9.4 Mass Determination and Event Weights . . . . . . 200

9.5 Sielecting Kaon and Proton Pairs . . . . . , . 205

9.6 Background Estimates . . . . . . . . . . 206

9.7 Analysis of the $K^{+} K^{-}$Mass Spectrum . . . . . . 207
9.7.1 Data Reduction and Normalization . . , . . , 207

9.7.2 Model for $\gamma \gamma \rightarrow K^{+} K^{-}$. . . . . . . . . . 209

9.7.3 Fitting the Invariant-Mass Spectrum . . . . . . 213

9.8 Measurement of $\gamma \gamma \rightarrow p \bar{p} \quad$. . . . . . . . . . . . 216

10. Conclusions . . . . . . . . . . . . . 218

10.1 The QED Channel - . . . . . . . . . 218

10.2 Cross Section for $\gamma \gamma \rightarrow \pi^{+} \pi^{-} \quad$. . . . . . . . . . 218

10.2.1 Measurements from Untagged Data $\left(Q^{2} \approx 0\right)$. . . . 218

10.2.2 Measurements from Tagged Data $\left(\overline{Q^{2}}=0.44 \mathrm{GeV}^{2}\right)$. . 225

10.3 Measurement of the $f^{\prime}$ Two-Photon Width . . . . . 226

10.4 Cross Section for $\gamma \gamma \rightarrow p \bar{p}$. . . . . . . . . . . . 229

Appendix A. Integration of the EPA spectrum . . . . . . 231

Appendix B. Monte Carlo Integration and Event Generation . . . 235

B.1 The Monte Carlo Method . . . . . . . . . 235

B.2 Monte Carlo Integration of the EPA Spectrum . . . . 238

B.3 Including the Cross Section for $\gamma \gamma \rightarrow 1^{+t^{-}}$. . . . . 240

B.4 Monte Carlo for the Single-Tag Luminosity Function . , . 241

B.5 Calculations Without EPA . . . . . . . . . . 242

Appendix C. Unfolding Methods for Experimental Distributions . $\quad 247$ REFERENCES . . . . . . . . . . . . . 254 


\section{List oi Trbles}

1.1 Particle properties for the tensor misons. - . . . . . . 6

3.1 Monte Carlo predictions for the momentum resolution . . . 37

4.1 A parameterization of the electron identification efficiency. . . 60

4.2 A parameterization of the Cerenkov latch effieieney. . . . 62

4.3 A parameterization ot the shower counter latch efficiency. . . 69

4.4 List of the final cuts for $e^{+} e^{-} \rightarrow e^{+} e^{-} e^{+} e^{-} . \quad . \quad . \quad . \quad . \quad 71$

4.5 List of the sources of error in the luminosit; . . . . . . 92

4.6 List of the sources of error in the luminosity . . . . . . . . 94

5.1 List of some of the cuts for the $\pi^{+} \pi^{-}$anslysis. , , . . 105

5.2 Sources of error in the normalization . . . . . . . 112

5.3 Sources of error in the normalization . . . . . . . . 121

8.1 Resulte of fitting the $\pi^{+} \pi^{-}$model . . . . . . . . . 176

8.2 Results of fitting the Mennessier model . . . . . . 181

8.3 Fit of the complete model . . . . . . . . . 184

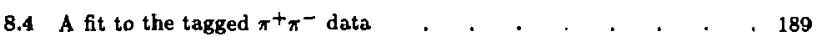

9.1 A fit of the model for $\gamma \gamma \rightarrow K^{+} K^{-} \quad$. . . . . . . . 215

9.2 Contributions to the error on $\mathrm{BR}\left(f^{\prime} \rightarrow K \bar{K}\right) \cdot \Gamma_{f^{\prime} \rightarrow 77} \quad$. , 216

10.1 Summary of published measurements of $\Gamma_{/ \rightarrow \gamma \gamma}$. . . , 224

10.2 Comparison of measurements and limits on $\Gamma_{f^{\prime} \rightarrow \gamma \gamma}$. . . 228

\section{List of Figures}

1.1 Production of non-leptonic final states in $e^{+} e^{-}$collisions. . . 2

1.2 Angular distributions for the decay of a tensor meson . . . 9

$1.3 S U(3)$ representations formed by the $u, d, s$ quarks . . . . 13

2.1 A collision of two bremsstrahlung photons. . . . . . 18

2.2 An electron-photon vertex with external electron lines. . . . 21

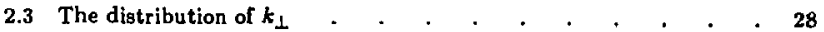

3.1 Longitudinal cross section of the DELCO detector. . . . 31

3.2 Transverse cross section of the DELCO detector. . . . . 32

3.3 Drift chamber cell confrgurations. . . . . . . . 34

3.4 The measured $K_{\mathrm{s}}$ mass distribution . . . . . . . . 38

3.5 The measured distribution of $k_{\perp}$. . . . . . . . . 39

3.6 The optical system of a Cerenkov counter cell. . . . . 41

3.7 The efficiency of the pattern recognition . . . . . . 48

4.1 The types of diagrams contributing to $e^{+} e^{-} \rightarrow e^{+} e^{-} l^{+} l^{-}$. . . . 52

4.2 The angular acceptance of the barrel shower counters , , , 54

4.3 The distribution of Cerenkov time residuals from electrong. . . $\quad 57$

4.4 The distribution of Cerenkov pulse height for electrons. . . 58

4.5 The efficiency for electron identification . . . . . . 60

4.6 The Cerenkov latch efficiency a . . . . . . . . 62

4.7 The shower counter layers-1,2 biased latch efficiency . . . 64

4.8 The shower counter layer-3 biased latch eificiency . . . . 65

4.9 The shower counter latch efficiency . . . . . . . . . 66

4.10 The measured trigger-biased shower-counter latch efficiency $\quad 67$

4.11 The biased shower counter latch efficiency a . . . . . 68

4.12 The electron-pair invariant mass distribution . . . . . 73

4.13 The electron-pair angular distributions . . . . . . 74

4.14 The lectron-pair invariant-mass distribution . . . . . 77

4.15 The velocity, $\bar{\beta}$, of the $\gamma \gamma$ electron pair . . . . . . . 79 
4.16 The center-of-mass electron-pair angular distribution . . . 80

4.17 The luminosity counter energy deposit. . . . . . . . . 82

4.18 The $k_{\perp}$ distribution for tagged events. . . . . . . 84

4.19 Electron-pair invariant mass for tagged events . . . . 85

4.20 Angular distribution for e-pairs from tagged events. . . . 800

4.21 The fractional energy loss of electrons . . . . . . . . 90

5.1 The Cerenkov in-time raw pulse height distribution . . . 99

5.2 The Cerenkov out-of-time raw pulse height distribution . . . 100

5.3 Cosmic-ray rejection. . . . . . . . , . , $10 z$

5.4 The $z$ position of the interaction point. . . . . . . . 103

5.5 The Monte Carlo shower counter latch efficiency . . . . 107

5.6 The resolution of the shower-counter edge for electrons. . . 108

5.7 The pion-pair and muon-pair invariant mass. . . . . . 113

5.8 The $\eta^{\prime} \rightarrow p^{0} \gamma$ background $\pi^{+} \pi^{-}$mass spectrum . . . . 117

5.9 The $K^{+} K^{-}$plus pp background . . . . . . . . . 118

5.10 Transverse momentum cut for tagged dats. . . . . . . 119

5.11 The invariant mass spectrum for tagged non-electron pairs. . 123

6.1 Resolution of the shower counter edge . . . . . . . 126

6.2 The shower counter latch efficiency for non-electrons . . . 128

6.3 HETC calculation of the shower counter latch efficiency . . 130

6.4 The pion-pair trigger efficiency . . . . . . . . . 132

7.1 Leading-order QCD diagrams . . . . . . . . . . 134

7.2 The complex $s$ plane $\quad . \quad$ - . . . . . . . . . . . 143

7.3 The Feynman diagrams for the Born term . . . . . 144

7.4 The $I=0, J=2 \pi \pi$ phase shift . . . . . . . . . 147

7.5 The effect of $\rho$ exchange on the $\pi^{+} \pi^{-}$continuum . . . . 150

7.6 The Mennessier model at $\theta=\pi / 2 \quad$. . . . . . . . . . . 159

7.7 Prediction for the $\gamma \gamma \rightarrow \pi^{+} \pi^{-}$cross section , . . . . 160

7.8 Effect of the energy dependence of the $f$ resonance . . . . . 162
7.9 The cfice of tequiring unitarity for the $D$-wave . . . . 164

8.1 The background-subtracled $\pi^{+} \pi^{-}$invariant-mass spectrum . . 177

8.2 Best simultaneous fit to the $W$ and $\cos \theta_{\mathrm{cms}}$ distributions , , 185

8.3 Complete fit of the tagged $\pi^{+} \pi^{-}$data . . . . . . . 190

9.1 Energy deposit of kaons . . . . . . . . . 194

9.2 The pulse-neight dependence of the resolution . . . . . 195

9.3 The time-of-flight phototube time resolution . . . , . 196

9.4 Histogram of the time consistency . . . . . . . . 198

9.5 The time-of-flight resolution. . . . . . . . . 199

9.6 The momentum versus the inverse of the measured velocity . . 200

9.7 The time-of-flight $m^{2}$ for non-eleciron pairs. . . . . . 202

9.8 Scatter plot of time-of-fight $m_{+}^{2}$ us $m_{-}^{2}$. . . . . . . . 204

9.9 The $p \bar{p}$ and $K^{+} K^{-}$event weights . . . . . . . 205

9.10 The detection efficiency for $K^{+} K^{-}$. . . . . . . 208

9.11 The measured $K^{+} K^{-}$invariant-mass spectrum. . . . . 210

9.12 Predictions for the $K^{+} K^{+}$mass spectra. , , , , . 212

10.1 The measured differential cross section . . . . . . 220

10.2 The measured differential cross section for $\cos \theta_{\mathrm{cms}},{ }, 221$

10.3 The cross section for $\gamma \gamma \rightarrow \pi^{+} \pi^{-} \quad$. . . . . . . . 222

10.4 The measured $Q^{2}$ dependence of $\Gamma_{f \rightarrow \tau \gamma}$. . . . . , 226

10.5 Predictions for the resonant contribution to $\sigma_{\gamma \gamma \rightarrow K+K^{-}} \quad$. 227

10.6 $S U(3)$ quark model predictions for the ratios of two-photon widths 229

A.1 The invariant-mass differential cross section for $e^{+} e^{-}$pairs $\quad . \quad 234$

B.1 Six diagrams for the process $e^{+} e^{-} \rightarrow e^{+} e^{-1+l^{-}}$. . . . . 243

B.2 Two calculations of the $e^{+} e^{-}$invariant-mass distribution. . . 245

B.3 Two calculations of the $e^{+} e^{-} k_{\perp}$ and $\theta_{\text {cms }}$ distributions. . . 245

C.I A sequence of 20 cubic B-splines. . . . . . . . . 248 


\section{The Two-Photon Particle Production Procese}

\subsection{INTHODUCTION}

The quantum theory of electrodynamics (QED) predicts that photons, the quanta of the electromagnetic field, will interact with each other through the exchange of virtual electron paira-a phenomenon which necessarily is absent in the classical field theory of Maxwell. However, such processes are not observed from usual sources of electromagnetic radiation because the cross sections are prohibitively small except at photon energies well above the electron mass. But in the energy realm of modern accelerators, interest in nhoton-photon interactions goes well beyond the relatively simple QED processes. Photons interact with many particles other than electrons, so at high energies many possibilities are experted for two-photon interactions, with hadrons as well as leptons in the final state.

Unfortunately, there are no sources of free, massless photons in the energy ranges of interest to high energy particle physics which are intense enough to produce observable photon-photon collisions. Instead, such collisions always have been studied indirectly as interactions of virtual photons. The first methods to be used, such as the Primakov effect for two-photon production of $\pi^{0}$ and $\eta$, involve the interaction of high energy photons with stationary atoms. An incident photon interacts with a virtual photon from the electromagnetic field around the atomic nucleus to produce a $\pi^{0}$, for example. A more recent method, and the one most widely used, involves the use of the high intensity electron-positron colliding beams available in present-day storage rings.

The most simple process by which two oppositely charged electrons can interact to produce a hadronic final state is through annihilation into a single virtual photon with an energy equal to twice the beam energy. That is contrasted with the process of interest to two-photon physics, in which a photon is radiated by each beam. Sehematic diagrams for both processes are shown in Fig. 1.1. The second is of higher order in the small electron-photon coupling than the first, but the cross sections for two-photon processes can be very large in spite of that.

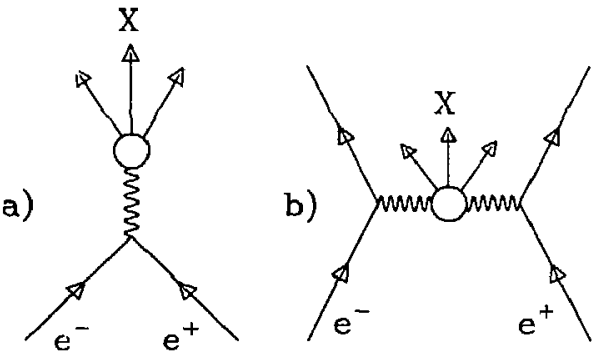

Figure 1.1. Production of non-leptonic final states in $e^{+} e^{-}$collisions. Two possibilities: (a) single-photon production and (b) two-photon production.

The reason is that single-photon production requires a spacelike photon with a mass equal to twice the beam energy, while the two-photon process proceeds with timelike photons of generally lower energy and very low mass. Thus the two-photon processes can have relatively large cross sections in certain kinesuatic regions. In particular, the two-photon process favors the production of statog $X$ which have much lower energies than are avaiable in single-photon annihilation. Also, the two processes produce ztates with differing quantum numbers, so th.ey are in many respects complimentary. For example, the two-pnoton states have positive $C$ parity, while for single-photon production the $C$-parity is negative. Also, a aingle photon always has spin-one and parity negative, while two photons can couple to resonances with a variety of spin-parity combinations.

Since two-photon collisions at $e^{+} e^{-}$storage rings are dom nated by interactions of photons of low invariant mass (small $Q^{2}$ ), they resemble the interaction of two real photons. The electron beams act essentially as intense sources of bremsstrahlung radiation, which interact as would two beams of high energy photons. This line of thought is developed mathematically in Chapter 2, where we 
calculate the spectrum of colliding photon pairs expected from an $e^{+} e^{-}$storage ring and thereby justify the statements made here regarding the scales of $Q^{2}$ and energy involved in the photon-photon collisions.

DELCO is an experiment which has taken data at the PEP electron-positron siorage ring of the Stanford Linear Accelerator Center (SLAC). The emphasis of the detector is on tracking and identification of charged particles. In the momentum range pertinent to two-photon physics, electrons are identified by a threshold gas Cerenkov counter, and charged kaons and protons are identified by time-of-flight measurements. For both identification methods, a measurement of the particle momentum is essential, and that is made by drift chambers positioned within a magnetic fleld. A mote complete description of the detector and its performance is given in Chapter 3.

The detector components used for particle tracking and identification cover about $60 \%$ ot the solid angle, in a region centered about a plane perpendicular to the beam line. That is not sufficient for otudies of inclusive particle production, especially since the two-photon state is preferentially boosted along the direction of the beam, causing most of the particles to escape out the ends of the detector. The detector is suitable for studies of exclusive states of low multiplicity, where all of the particles produced from the two-photon interaction are detected and identified. In particular, the ability of the detector to identify low momentum electrons gives special advantages in the atudy of two-photon interactions which produce only two stable particles in the final state. In this thesis, the reaction channels which are studied are

$$
\begin{aligned}
e^{+} e^{-} & \rightarrow e^{+} e^{-} e^{+} e^{-}, \\
e^{+} e^{-} & \rightarrow e^{+} e^{-} \pi^{+} \pi^{-}, \\
e^{+} e^{-} & \rightarrow e^{+} e^{-} K^{+} K^{-}, \\
\text {and } & e^{+} e^{-} \rightarrow e^{+} e^{-} p \bar{p} .
\end{aligned}
$$

For the QED channel, the kinematic distributions measured from data are compared in shape with theoretical calculations. Also, the QED channel is used as an accurate normalization for subtraction of the $e^{+} e^{-} \rightarrow e^{+} e^{-} \mu^{+} \mu^{-}$background from the remaining data and for measurements of the cross sections of the hadronic channels. In the $\pi^{+} \pi^{-}$channel the two-photon partial width of the $f(1270)$ resonance is measured, and in the $\mathrm{K}^{+} \mathrm{K}^{-}$channel the two-photon partial width of the $f^{\prime}(1525)$ is measured. As the measurements of these partial widths is a principle object of this research, this chapter concentrates on discussing the meaning of the two-photon partial width and its theoretical significance. Chapter 7 elaborates at length on the specific theoretical models used in the analysis of the $\pi^{+} \pi^{-}$channel.

\subsection{Hadron Production from Photon-Photon INTERACtIONS}

Since hadrons are believed to be composed of point-like charged quarks, the interactions of photors with hadrons should ultimately be described by a QED type of coupling of photons to spin- $\frac{1}{2}$ point fermions. The interactions of the quarks among themselves should then be described by Quantum Chromodynamics (QCD), in which the strong interactions are mediated by vector bosons called gluons, with additional small contributions from electromagnetic and weak effects. However, we know that hadrons are tightly bound within regions with dimensions smaller than 1 fermi $=5 \mathrm{GeV}^{-1}$. Therefore, it is clear than a photon must have an energy much greater titan $0.2 \mathrm{GeV}$ before it could effectively resolve any of the detailed structure of a hadron. $\dagger$ Otherwise, it may be Sest to consider a physical description of photons coupling to point hadrons, with the addition of some form factor to describe deviations from point-like behavior. In principle, the form factor might be calculable from QCD; however, all that is possible at this point in time is to calculate perturbative expansions which are valid only at very short distances in space-time. Thus it is consistent to calculate scattering amplitudes in which photons couple to quarks and quarks interact through gluon exchange, but only if

4 That is not to oay that the badron atructure cannot havo a large effect on the atrength of the coupling to photons. In fact, it is important, especially when the hadron is neutral, as we will see in Section 1.5 . 
all of that takes place on a very small time and distance scale. What that means is that the wavelengths of the photons must be small on the scale of a hadron, and there must be a large momentum transfer between photons and quarks.

Even then, after such a hard-scattering interaction occurs, the quarks remaining in the final state continue to interact over a long time interval, and the interaction, in fact, grows stronger as they separate from the point of collision with the photons. So one can not get away from the fact that the low-energy non-perturbative strong interaction effects must be modeled phenomenologically. The best that one can hope for is a factorization of the hard-scattering effects from the non-perturbative part, so that they may be dealt with separately, and even measured separately to some extent. That has been done for the production of hadron pairs by two real photons by Brodsky and Lepage ${ }^{1}$ and extended to include virtual photons by Gunion, Millers, and Sparks? If the hadron pairs are observed at sarge angles with respect to the incoming beams, which is experimentally necessary for most present experiments, anyway, then for sufficiently large twophoton invariant mass, the photon energies and the momentum transfer are large enough that the perturbation expansion and factorization make sense. The first available experimental results indicate agreement, of low statistical precision, with the theory for pion pairs ${ }^{3,4}$ and kaon pairs, for pair masses as low as $1.5 \mathrm{GeV}$ for kaons and $2.0 \mathrm{GeV}$ for pions.

In any case, DELCO is restricted by its methods of particle identification to pair masses below $2 \mathrm{GeV}$ (or $3 \mathrm{GeV}$ for proton pairs). Therefore, in the DELCO data neither do the photons have enough energy to finely resolve hadronic structure nor is QCD perturbation theory valid. Tristead, the hadrons and hadron resonances must be considered to be the elementary particles, and their couplings to photons and to other hadrons are described according to phenomenology whirh is well known from low-energy (by today's standards) hadron physics. For the $\pi^{+} \pi^{-}$ final state, the relevant phenom anology is presented in Chapter 7.

\subsection{Resonance Formation}

The energy range accessible to DELCO ig, in fact, dominated by resonant effects from the $f(1270)$ in the $\pi^{+} \pi^{-}$channel and the $f^{\prime}(1525)$ in the $K^{+} K^{-}$ channel. These resonances both represent tensor mesons of zero isospin and have been studied extensively in purely hadronic interactions. They are closely related to an isovector tensor meson called the $A_{2}(\mathbf{1 3 2 0})$. Some of the information already known about the three is shown in Table 1.1.

Table 1.1. Particle properties for the tensor mesons. The data are taken from Ref. 6 and represent averages of many experimental results, except for the $f^{\prime}$ branching ratio to $\gamma \gamma$, which is from Ref. 7 , the only published measurement to date. No DELCO results are included.

\begin{tabular}{|c|c|c|c|c|c|}
\hline Particle & $J^{G}\left(J^{P}\right) C$ & $\begin{array}{l}\text { Mass } \\
(\mathrm{MeV})\end{array}$ & $\begin{array}{l}\text { Width } \\
\text { (MeV) }\end{array}$ & $\begin{array}{l}\text { Decay } \\
\text { mode }\end{array}$ & $\begin{array}{c}\text { Fraction } \\
\%\end{array}$ \\
\hline$f$ & $\mathbf{0}^{+}\left(\mathbf{2}^{+}\right)+$ & $\begin{array}{r}1274 \\
\pm 5\end{array}$ & $\begin{array}{r}178 \\
\pm 20\end{array}$ & $\begin{array}{c}\pi \pi \\
\pi^{+} \pi^{-} \pi^{+} \pi^{-} \\
k \bar{K} \\
\gamma \gamma\end{array}$ & $\begin{array}{c}84.3 \pm 1.2 \\
2.9 \pm 0.4 \\
2.9 \pm 0.2 \\
0.0015 \pm 0.0002\end{array}$ \\
\hline$A_{2}$ & $1^{-}\left(2^{+}\right)+$ & $\begin{array}{r}1318 \\
\pm 5\end{array}$ & $\begin{array}{r}110 \\
\pm 5\end{array}$ & $\begin{array}{c}\rho \pi \\
\eta \pi \\
\omega \pi \pi \\
K \bar{K} \\
\pi \gamma \\
\gamma \gamma\end{array}$ & $\begin{array}{c}70.1 \pm 2.2 \\
14.5 \pm 1.2 \\
10.6 \pm 2.5 \\
4.9 \pm 0.8 \\
0.27 \pm 0.06 \\
0.00075 \pm 0.00016\end{array}$ \\
\hline$f^{\prime}$ & $0^{+}\left(2^{+}\right)+$ & $\begin{array}{r}1525 \\
\pm 5\end{array}$ & $\begin{array}{r}70 \\
\pm 10\end{array}$ & $\begin{array}{l}K \bar{K} \\
\gamma \gamma\end{array}$ & $\begin{array}{c}\text { dominant } \\
0.00016 \pm 0.00006\end{array}$ \\
\hline
\end{tabular}

The cross section for producing a resonance $X$ from two photons is proportional to its two-photon partial width $\Gamma_{\boldsymbol{\gamma}}$ - Also, the probability for a resonance to decay into two photons is proportional to $\Gamma_{\gamma \gamma}$. So if the total width of the 
resonance is represented by $\Gamma$, then the two-photon partial width is related to the branching ratio to two photons by $\Gamma_{\eta\urcorner}=\mathrm{BR}(X \rightarrow \gamma \gamma) \cdot \Gamma$. Thus the two-photon partial widths of the tensor mesons are, according to Table 1.1,

$$
\begin{aligned}
\because \quad \Gamma_{f-\gamma \gamma} & =2.7 \pm 0.3 \mathrm{keV} \\
\Gamma_{\Lambda_{2} \rightarrow \gamma \gamma} & =0.82 \pm 0.18 \mathrm{keV} \\
\Gamma_{f^{\prime} \rightarrow \gamma \gamma} & =0.11 \pm 0.0^{\circ}
\end{aligned}
$$

Tl e:le values all have been measured by two-phois.

-Lion at $e^{+} e^{-}$storage rings, so they all suffer from the same kinds of systematic effects as do the DELCO measurements. For example,

- The measuremente are made with virtual photons, so an extrapolation must be made to $Q^{2}=0$. We will see that for the usual experimental situation this effect is small.

- Generally the resonance is not prorluced in isolation. Usually there is substantial continuum production which interferes with the resonant effects, and it must be modeled roperly in order to extract the resonant cross section.

- The tensor mesons have widths which are large on the Bcale of the typical experimental resolution. Therefore, the energy dependence of the cross section must be appropriately modeled, leading to partial widths which are not constant with energy. One must be careful to understand how the final number ziven for the partial width is defined. This is closely related to the previous point-interference with a background would be no problem if the width were negligibly small.

- A tensor meson may be produced from photon pairs with a total helicity of either zero or two with respect to the axis along which the photons travel in the center-of-mess system. Therefore, the angular distribution of the decay products is not known a p:iori but depends on dynamics of the production. This problem is discussed at length in the following section.
- Moat experiments have not been optimized for studying the relatively low energy two-photon production processes. This complicates the always difficult problem of understanding the detection efficiency.

\subsection{Angular Distributions of Tensor Meson Decays}

Consider a spin-2 meson in its rest frame with spin component $\lambda=0, \pm 1, \pm 2$ in the $z$ direction. When it decays into two spin- 0 mesons, the angular distributions are given by the squares of $d$-functions, $d_{\lambda 0}^{J}(\theta)$, where $\theta$ is the angle between the momentum of one of the decay products and the $z$ axis. Figure 1.2 shows plots of the three angular distributions, using

$$
\begin{aligned}
& d_{00}^{2}(\theta)=\frac{3}{2} \cos ^{2} \theta-\frac{1}{2} \\
& d_{10}^{2}(\theta)=-\sqrt{\frac{3}{2}} \sin \theta \cos \theta \\
& d_{20}^{2}(\theta)=\frac{\sqrt{6}}{4} \sin ^{2} \theta .
\end{aligned}
$$

Also shown is the shape of the experimental acceptance for pairs produced at the angle $\theta$ in the meson rest system, assuming that the mesons are produced by $\gamma \gamma$ collisions in an $e^{+} e^{-}$storage ring and observed by a detector with a laboratory acceptance of $\cos \theta_{\text {leb }}<0.6$ (appropriate for DELCO).

If the experiment is done with photons which are almost real, as is usually the case, ithen tensor mesons can be produced only with helicity $\lambda=0$ or 2 , assuming an axis of quantization parallel to the photon momenta in the center-ofmass system. Unfortusately, the angular distributions for these two possibilities are rather similar in shape in the xperimental region of greatest acceptance, so the experiment is severely limited in determining the fraction of each component in the data. If that fraction is incorrect, then it is clear that an extrapolation to the fu'l solid angle, which is necessary to determine the two-photon partial width of the meson, can be greatly in errer, since the two angular distributions are not at all similar near $\cos \theta= \pm 1$. Therefore, it is useful to have some knowledge of 


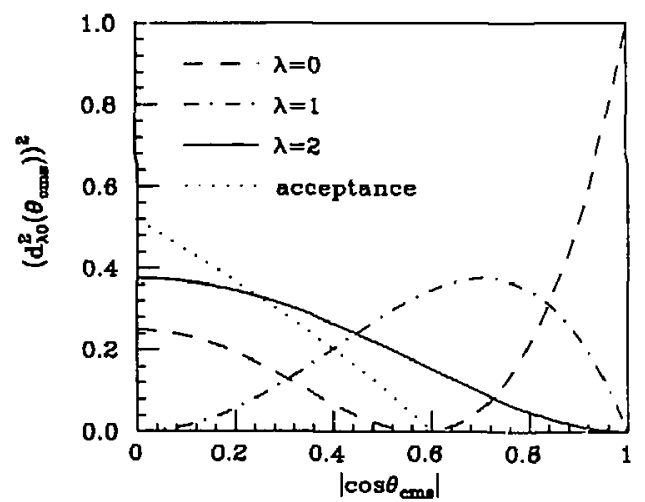

Figure 1.2. Angular distributions for the decay of a tensor meson into two spin-zero mesons for helicities $\lambda=0,1,2$. Also shown is the shape of the acceptance of the DELCO detector.

the ratio of the helicity amplitudes either from theory of from other experiments with greater angular acceptance.

The Crystal Ball collaboration, using a detector with a maximum acceptance of $98 \%$ of the solid angle, measured the angular distribution for $\gamma \gamma \rightarrow f \rightarrow \pi^{0} \pi^{0}$ within the limit $|\cos \theta|<0.9$ and determined that ${ }^{\circ}$

$$
\frac{\Gamma_{77}^{\lambda=0}}{\Gamma_{77}^{\lambda=2}}=0.12 \pm 0.39
$$

Theoretica: limits are even more stringent. In Ref, 10, elastic $\gamma \gamma$ scaltering is considered, and a sum rule is derived from fixed $t$ dispersion relations by invoking crossing symmetry. When partial wave expansions are inserted into the sum rule, with the partial wave amplitudes approximated by a series of narrow resonance contributions, then the following inequality is obtained:

$$
\sum_{T} \frac{g_{T \rightarrow 7 \gamma}^{2}(\lambda=2)}{m_{T}^{8}} \geq 6 \sum_{T} \frac{g_{T}^{2} \rightarrow \gamma_{\gamma}(\lambda=0)}{m_{T}^{8}}+\text { positive } J^{P}=4^{+} \text {contributions, }
$$

where the sums are over all of the tensor mesons. Becsuse of the factors of $1 / \mathrm{m}_{T}^{8}$, only the $f, f^{\prime}$, and the $A_{2}^{0}$ contribute significantly, and from quark-parton-model predictions, one expects the main contribution to be from the $f$. Therefore, the $f$ partial width into $\gamma \gamma$ with kelicity-zero is expected to be suppresned by at least a factor of six relative to the helicity-two partial width.

The authors of Ref. 8 and Ref. 5 claim that a six-tu-one : 1ppression of the helicity-zero amplitude may be $c$ iculated from Clebsch-Gor: an vector coupling coefficients. They show that if the fry aystem is presumed o have an angular momentum given by only the sum of the photon spins, thet: the $J=2, M=0$ component of the ry system is eix times smaller than the $J=2, M=2$ component. But that says nothing about how strongly the meson couples to the two helicity amplitudes. The two-photon width is a dynamical quantity related to the censor meson structure.

We describe the photon pair and pseudoacalar-meson pair by helicity states because that is a proper and convenient description of a pair of relativistic particles. For $J=2$ there are five such states, but symmetry requires that the coupling be the same for $+\lambda$ as for $-\lambda$, leaving only three independent amplitudes for an interaction. In a nonrelativistic, case, one might find it convenient to make an $L S$ decomposition and describe the two-particle state by the total apin a and the orbital angular momentum $l$, such that $\vec{j}=\vec{s}+\vec{l}$. One exsily can see that for $J=2$ there are five posoible combinations of $a$ and $l$. Symmetry must again reduce the number of independent couplings to three, but it no longer is so obvious. Hence we talk in this case of some sort of orbital angulas momestum amplitudes rather than helicity amplitudes. One could make the dynamical assumption that only $l=0$ contributes, but some sort of justification must be given. Then the 
number of amplitudes would be reduced to one. But when the relativistic regime is approached, it does not seem to be of an" greater advantage to make an ad hoe assumption that $l=0$ than to assume that $\lambda=2$. Furthermore, it is a fact that an LS decomposition cannot be made for a pair of massless particles such as photons (see Section 7.1).

Finally, let us consider what modifications of these ideas are necessary for an experiment done with virtual photons. We wish to measure the two-photon widths of tensor mesons for real photons, which requires a small extrapolation of the result obtained from seattering of photons generated by collicing electron beams. For real photons, the helicity-one amplitude is exactly zero, but it is possible for experiments done with quasi-real photons to see some contribution from helicity-one in addition to the helicity-zero and helicity-two contributions. In making the extrapolation to real photons, one would want to isolate the helicityone contribution and drop it. We will not be concerned about this problem, though. First of all, the $\gamma \gamma$ collisions observed by DELCO are from photons which are sufficiently close to being real that the helicity-one component must already be negligibly small. $\uparrow$ Second, it is clear from Fig. 1.2 that the acceptance of DELCO is amall for the helicity-one component, relative to the others, so not much of that component would be observed even if it were produced.

\subsection{UNTARY SYMMETRY AND THE TENSOR MESON NONET}

The theoretical importance of the two-photon width of a meson stems from the general utility of electromagnetic probes. At a fundamental level, photons are known to couple only to charged particles, and the nature of the coupling is known in full detail from QED. Therefore, the only missing information about how the meson is produced from photons is the detailed structure of charged currents within the meson. The structure depends upon the composition of the meson and the strong interactions of its constituents, and it is what is of theoretical interest.

i That is, for the untagged experiment (ace Chapter 2).
The most basic theoretical model is the quark-parton model, which assumes that each meson is composed of two spin- $\frac{1}{2}$ quarks of flavor $u$, d, or s. The only dynamical assumption made is that of (slightly broken) $S U(3)$ symmetry under transformations of isospin and hypercharge. The $u, d$, and quarks form a 3 representation of $S U(3)$, and their antiparticles form a $\overline{\mathbf{s}}$ representation, as shown in the weight diagrams of Fig. 1.3. The mesons are formed from combinations of quark and antiquark. Superimposing the two weight diagrams immediately generates the nine possible combinations of the $I$ and $Y$ quantum numbers, but there is an additional quantum number, spin, which must be considered. Spin singlet states are formed when the quark and antiquark have their spins anti-parallel, yielding the pseudoscalar nonet $\left(\eta, \eta^{\prime}, \pi, K\right)$, while the vector nonet $\left(\phi, \omega, \rho, K^{\bullet}\right)$ is formed when the spins are parallel. The intrinsic apin of the quarks should not be the only source of angular momentum, so we expect higher apin states. The $J=2$ nonet has been observed, and the particles of which it is composed are shown in Fig. 1.s. We can consider these mesons to be companed of massive spin- $\frac{1}{2}$ quarks in a $P$-wave atate of relative angular momentum.

The $3 \otimes \overline{3}$ representation of Fig. $1.3 \mathrm{may}$ be reduced to 8 combination of octet and singlet irreducible representations, $\theta \oplus 1$. The charge neutral $A_{2}$ obviously is a member of the octet. It is represented in terms of the direct product quark-pair states by

$$
\left|A_{2}, Q=0\right\rangle=\frac{1}{\sqrt{2}}(|d \bar{d}\rangle-\mid u(u)) .
$$

One of the other neutral states is in the octet, while the last forms the singlet. They are respectively

$$
\begin{aligned}
& \left|f_{8}\right\rangle=\frac{1}{\sqrt{6}}(|u \bar{u}\rangle+|d \bar{d}\rangle-2|s \bar{s}\rangle) \\
& \left|f_{1}\right\rangle=\frac{1}{\sqrt{3}}(|u \bar{u}\rangle+|d \bar{d}\rangle+|s \bar{s}\rangle) .
\end{aligned}
$$

The fact that the mesons of the octet in Fig. 1.3 do not all have the same mass is a clear indication that $S U(3)$ symmetry cannot be exact. The strange mesons 

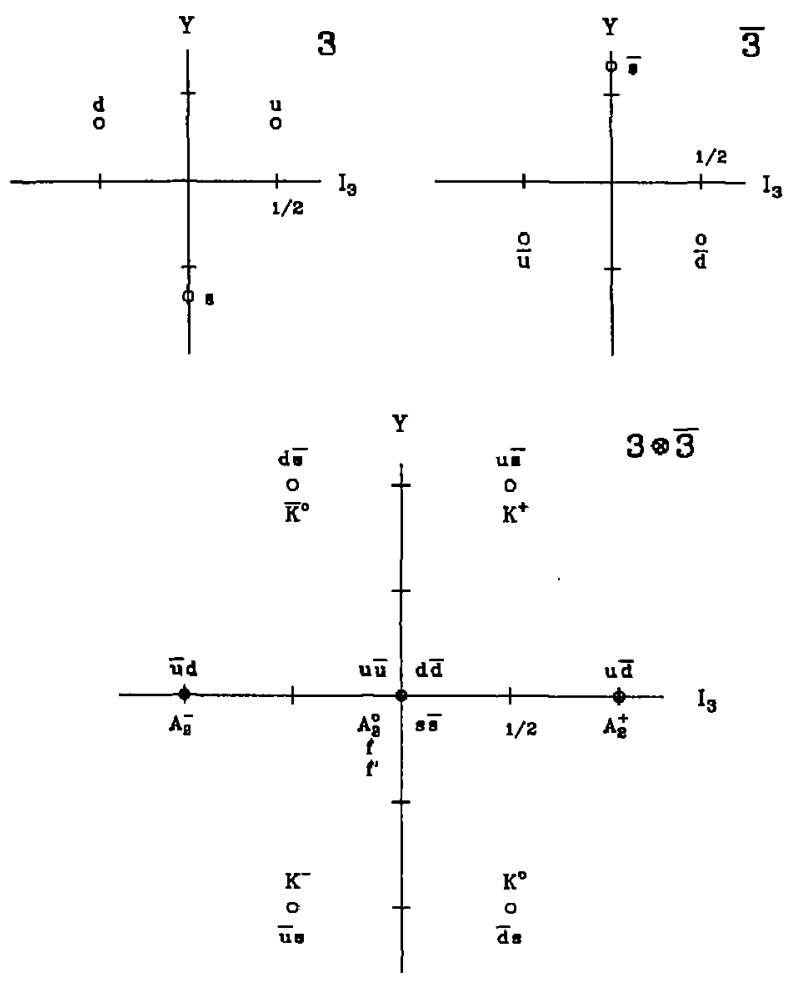

Figure 1.3. $S U(3)$ representations formed by the $u, d, s$ quarks and their antiparticles. $J_{3}$ is the third component of isospin, and $Y$ is the hypercharge. The electric charge is given by $Q=I_{3}+Y / 2$. The $K$ states shown in the octet representation are the $K^{\bullet}(1430)$. generally have larger masses than the others, which suggests that the $s$ quark is more massive than the other two. In that case, the symmetric combinations of Eqn. 1.6 are unlikely to be realized in nature. In fact, from the branching ratios to $K \bar{K}$ of the observed mesons $f$ and $f^{\prime}$, one might guess that the $f$ has almost no $s$-quark content, while the $f^{\prime}$ is almost completely composed of $s$ quarks. Let us define these physical states in terms of the $f_{s}$ and $f_{1}$ through a mixing angle $\theta$ :

$$
\begin{gathered}
|f\rangle=\cos \theta\left|f_{1}\right\rangle+\sin \theta\left|f_{B}\right\rangle \\
\left|f^{\prime}\right\rangle=-\sin \theta\left|f_{1}\right\rangle+\cos \theta\left|f_{8}\right\rangle .
\end{gathered}
$$

Then the situation called ideal mixing, in which the $f^{\prime}$ B purely $s 3$, occurs for $\theta=35.29^{\circ}$.

The coupling strength $g$ of $\gamma \gamma$ to a $q \gamma$ pair is proportional to the aquare of the quark charge: ${ }^{8}$

$$
\begin{array}{ll}
(q \bar{q} \mid \gamma \gamma) \propto e_{q}^{2} \cdot \Psi_{q}(0) & (S-\text { wave }) \\
\left(q q|\gamma \gamma\rangle \propto e_{q}^{2} \cdot \Psi_{q}^{\prime}(0)\right. & (P \text {-wave }),
\end{array}
$$

where $\Psi_{q}(0)$ and $\Psi_{q}^{\prime}(0)$ are the radial quark ivave function and its derivative at the origin. Assuming that the $\Psi_{q}^{r}(0)$ are independent of the $q \bar{q}$ favor, the $r 7$ couplings of the tensor mesons then are proportional to coherent sums, given by equations 1.5 and 1.6, of the squares of the quark charges:

$$
\begin{aligned}
& g\left(A_{2} \rightarrow \gamma \gamma\right) \propto \frac{1}{\sqrt{2}}\left(e_{d}^{2}-e_{u}^{2}\right)=-1 /(3 \sqrt{2}) \\
& g\left(f_{8} \rightarrow \gamma \gamma\right) \propto \frac{1}{\sqrt{6}}\left(e_{u}^{2}+e_{d}^{2}-2 e_{a}^{2}\right)=1 /(3 \sqrt{6}) \\
& g\left(f_{1} \rightarrow \gamma \gamma\right) \propto \frac{1}{\sqrt{3}}\left(e_{u}^{2}+e_{d}^{2}+e_{g}^{2}\right)=2 /(3 \sqrt{3}) .
\end{aligned}
$$

$S U(3)$ requires that the constant of proportionality in Eqn. 1.9 be the same for the $A_{2}$ and $f_{8}$. The singlet state is not necessarily related that simply to the actet states, however. If ouc does assume that the wave functions of the 
singlet and octet states are approximately equid, then that implies approximately equal binding energies." That in turn implies, when $S U(3)$ symmetry is broken by the $s$ quark mass, nearly ideal mixing of the $f_{B}$ and $f_{1}$. That is consistent with the observed tensor meson nonet (but not the pseudoscalar nonet), so it is reasonable to use Eqn. 1.9 along with Eqn. 1.7 to relate the ry widths of all three of the physical mesons. With the widths related to the coupling constants by $\Gamma_{\gamma \gamma} \propto m^{3} g^{2}$, we havet

$$
\begin{aligned}
& \frac{\Gamma\left(f^{\prime} \rightarrow \gamma \gamma\right)}{\Gamma(f \rightarrow \gamma \gamma)}=\left(\frac{m_{f^{\prime}}}{m_{f}}\right)^{3}\left(\frac{\cos \Theta-2 \sqrt{2} \sin \theta}{\sin \Theta+2 \sqrt{2} \cos \theta}\right)^{2} \\
& \frac{\Gamma(f \rightarrow \gamma \gamma)}{\Gamma\left(A_{2} \rightarrow \gamma \gamma\right)}=\frac{1}{3}\left(\frac{m_{f}}{m_{A_{2}}}\right)^{3}(\sin \theta+2 \sqrt{2} \cos \Theta)^{2}
\end{aligned}
$$

These relations give a reasonable fit to the data summarized in Eqn. 1.1, but we postpone any more consideration of them until the results of the DELCO experiment have been presented.

Finally, the importance of the $y y$ width goes well beyond these simple predictions of the quark model. First, one would not expect Eqn. 1.10 to be satisfled to a high degree of accuracy, but any clear deviations from it could provide useful experimental input into the problem of meson structure. Second, the $\gamma \gamma$ width has a better potential of being understood theoretieally than most nonperturbative phenomena associated with mesons. In fact, in the case of the $\pi^{0}$, the $\gamma \gamma$ width has been rigorously caleulated, ${ }^{12}$ due to the fortunate occurrence that it proceeds through a fermion loop, the axial anomaly, which is dominated by large loop momenta. Similar success has not yet been achieved for the tensor mesonsthe theoretical predictions for $\Gamma_{f \rightarrow 7 \gamma}$ range from about 1 to $20 \mathrm{keV} .{ }^{13}$ Nonetheless, the strength of the $\gamma \gamma$ coupling is an important fundamental parameter of any

\footnotetext{
† Kolanorki points out in Ref. 8 that there does not appear to be a rigorous proof that this expreavion for the phase space factor in valid-in some publications the width is taken to be proportional to meson nonet.
}

resonance, and except for the $\pi^{0}$, the best way to measure it is by $\gamma \gamma$ scattering at $e^{+} e^{-}$storage rings. 


\section{Two-Photon Interactions at $e^{+} e^{-}$Storage Ringe}

\subsection{KINEMATICS}

The object of this research is the study of cross sections for particle production from two-photon collisions. But the photons are produced over a range of momenta by electron beams, so the cross sections of interest always are convolved with a two-photon flux having a continuous energy spectrum. Also, the center of mass of the two-photon state generally is not the laboratory system but is boosted with a range of velocities relative to the laboratory. Thus the kinematics and the cross sections both are complicated, so it is essential to understand fully the scattering of the beam particles and production of the two photons before dealing with the interactions of the photons themselves.

First, some convertions must be established for the notation used to describe the kinematics. Figure 2.1 shows a sthematic diagram of the coliision with some of the variables to be used, which are listed in greater detail below: $\uparrow$

$\begin{array}{ll}p_{i} & \text { four-momenta of the elcetron beams }(i=1,2) . \\ E & \text { the storage ring beam energy. } \\ p_{i}^{\prime} & \text { four-momenta of the scattered electrons. } \\ E_{i}^{\prime}=p_{i}^{\prime 0} & \text { energies of the scattered electrons. } \\ m_{e}=p_{i}^{2} & \text { the electron rest mass. } \\ \varphi & \text { angle between the scattering planes. } \\ & \cos \varphi=\left(\vec{p}_{1} \times \vec{p}_{1}^{\prime}\right) \cdot\left(\vec{p}_{2} \times \vec{p}_{2}^{\prime}\right) \\ \theta_{i} & \text { polar angles with respect to the beam axis. } \\ \phi_{i} & \text { azimuthal angles. } \\ q_{i}=p_{i}-p_{i}^{\prime} & \text { momenta of the virtual photons. } \\ x_{i}=q_{i}^{0} / E & \text { energies of photons relative to the beam energy }\end{array}$

$\dagger$ Factors of $h$ and $c$ are omitted from formulas and kinematic expreasions, and the electron volt is used as a unit of mass, momentum, energy, and, often, of inverse length. Aloo, the term electron is uned to refer to both the electron and its antiparticle, in the asme way that the terms mion and pion commonly are used. Positron, or $\mathrm{e}^{+}$, is uned for the antiparticle when the distinction is important.

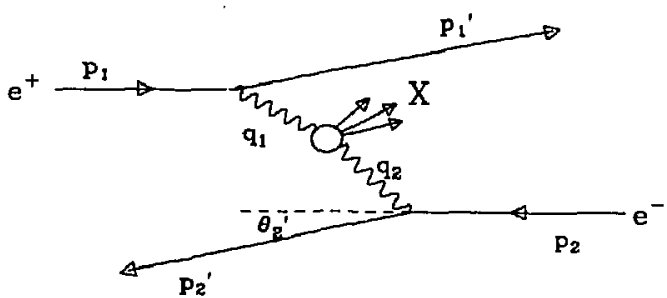

Figure 2.1. A collision of two bremsstrahlung photons. This schematic diagram shows the notation to be used for a description of the kinematics.
$Q_{i}^{2} \equiv-q_{i}^{2}$

$\varepsilon_{i}$

$k_{j}$

$k_{\perp}=\left|\vec{k}_{1 \perp}+\vec{k}_{2 \perp}\right|$

$E_{X}=\Sigma_{j} k_{j}^{0}$

$W=\sqrt{s}=\sqrt{\left(g_{1}+q_{2}\right)}$

$Z \equiv W / 2 E$

$y=\tanh ^{-1} \beta$

$u_{j}=\cos \bar{\theta}_{j}=\cos \theta_{\mathrm{cm}}$ is always positive.

polarization of the photons.

momenta of the $j$ th particle from the $\gamma \gamma$ system. total transverse momentum of the $\gamma \gamma$ system. total energy of the $\gamma \gamma$ system.

invariant mass of the $\gamma \gamma$ system.

rapidity of the $\gamma \gamma$ system in the lab frame.

$\tilde{\theta}_{j}$ is the polar angle of particle $j$ in the ry c.m.s.
The kinematics of the production of the two photons is determined by the four-momenta of the incoming and outgoing electrons. The $z$ axis is defined in the laboratory such that the two incoming beams travel along it with equal and opposite momenta and with energy $E(14.5 \mathrm{GeV}$ at PEP). The beams at PEP are unpolarized, so there can be no overall azimuthal dependence. Also, the polarization of the outgoing electrons is not measured, so the tatal number of variables to be used to describe the kinematics of the srattered electrons is only 
five. These are commonly defined in the laboratory system to be

- two energies, $E_{1}^{\prime}$ and $E_{2}^{\prime}$, of the scattered electrons,

- two angles, $\theta_{1}^{r}$ and $\theta_{2}^{\prime}$, that the momenta of the scattered beam electrons make with the beam axis,

- and one angle, $\varphi$, between the two scattering planes of the beam electrons.

From these variables, the kinematics of the $\gamma \gamma$ system are readily calculated.

The energies of the virtual photons are

$$
E_{\Upsilon_{i}}=E x_{i}=E-E_{1}^{\prime}
$$

and their invariant masses are

$$
\begin{aligned}
q_{i}^{2} & =\left(p_{i}-p_{i}^{\prime}\right)^{2} \\
& =2 m_{e}^{2}-2 E E_{i}^{\prime}\left(1-\sqrt{1-\left(m_{e} / E\right)^{2}} \sqrt{1-\left(m_{e} / E_{i}^{\prime}\right)^{2}} \cos \theta_{i}^{\prime}\right) .
\end{aligned}
$$

For all angles $\theta_{i}^{\prime}, q_{i}^{2}$ is less than zero, so the photons have spacelike momenta. Hence it is customary to define the positive quantities $Q_{i}^{2} \equiv-q_{i}^{2}$. Since $m_{e} / E \ll 1$ (3.5 $10^{-5}$ at $\left.P E P\right)$, most kinematic formulas can be simplifed by neglecting terms with it as a factor. One must be careful, though, in the region of very small-angle scattering, where the cross sections are the largest. In that case, $1-\cos \theta_{i}^{\prime} \approx \frac{1}{2} \theta_{i}^{\prime 2}$ is the order of $m_{e}^{2} / E^{2}$, so the electron mass cannot be neglected. In fact, substituting $\theta_{i}^{\prime}=0$ into Eqn. 2.2 gives the minimum possible value for $Q^{2}$ :

$$
Q_{i \min }^{2}=m_{e}^{2} \frac{x_{i}^{2}}{1-x_{i}}+O\left(m_{e}^{4} / E^{4}\right)
$$

On the other hand, if the ith scattered electron is detected at large angle (tagged), then it is possible to use the simple formula

$$
Q_{i}^{2}=4 E^{2}\left(1-x_{i}\right) \sin ^{2}\left(\frac{1}{2} \theta_{i}^{\prime}\right) \quad \theta_{i}^{\prime} \gg m_{e} / E
$$

For both tagged and untagged events, the invariant mass of the $\gamma \gamma$ system can be calculated accurately by neglecting all factors of $m_{e} / E$. Using $p_{i}^{\prime}=$ $E_{i}^{\prime}+O\left(m_{e}^{2} / E^{2}\right)$, we find

$$
\begin{aligned}
W_{\gamma \gamma}^{2} & =\left(q_{1}+q_{2}\right)^{2}=E^{2}\left(x_{1}+x_{2}\right)^{2}-\left(\vec{p}_{1}^{\prime}+\vec{p}_{2}^{\prime}\right)^{2} \\
& =4 E^{2}\left[x_{1} x_{2}-\frac{1}{2}\left(1-x_{1}\right)\left(1-x_{2}\right)\left(1+\hat{p}_{1}^{\prime} \cdot \hat{p}_{2}^{\prime}\right)\right],
\end{aligned}
$$

where $\hat{p}_{1}^{\prime} \cdot \tilde{p}_{2}^{\prime}=\cos \varphi \sin \theta_{1}^{\prime} \sin \theta_{2}^{\prime}+\cos \theta_{1}^{\prime} \cos \theta_{2}^{\prime}$ is the $\operatorname{cosine}$ of the angle between the two scattered electrons. Since in most cases the electrons scatter through small angles, yiclding photons with small $Q^{2}$, a good first approximation for untagged events is to use the simple formula $Z^{2} \equiv\left(W_{\uparrow y} / 2 E\right)^{2} \approx x_{1} x_{2}$.

\subsection{CROSS SECTIONS}

The diagram of Fig. 1.1-b divides naturally into three parts, two of which are electron-photon vertices and are understood completely. The well understood factors can be written out explicitly and reduced. Figure 2.2 shows a single electron-photon vertex with the QED Feynman rules included. $\dagger$ It contributes the factor $u(p, s)\left(-i e \gamma_{s}\right) \bar{u}\left(p^{\prime}, s^{\prime}\right)$ to the scattering amplitude, where $u(p, s)$ is the momentum-spact spinor wave function for a free electron with momentum $p$ and spin s. When the amplitude is squared in order to calculate the cross section, and the spins of the unpolarized electrons are summed over, a factor like

$$
\rho^{\mu \nu}=\frac{1}{-q^{2}} \sum_{s s^{\prime}} \bar{u}\left(p^{\prime}, s^{\prime}\right) \gamma^{\mu} u(p, s) \bar{u}(p, s) \gamma^{\nu} u\left(p^{\prime}, s^{\prime}\right)
$$

results, where $1 /\left(-q^{2}\right)$ is a normalization introduced for later convenience. Using twice the formula

$$
\sum_{a} u_{\alpha}(p, s) z_{\beta}(p, s)=\left(\frac{\phi+m_{e}}{2 m_{c}}\right)_{\alpha \beta},
$$

† The rules may be found in Appendix B of Ref. 14. The conventions used for the metric and other notation associated with field theory are those of Bjorken and Drell. 


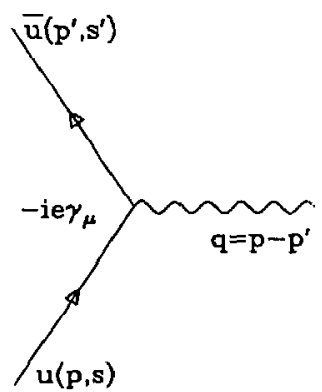

Figure 2.2. An electron-photon vertex with external electron lines.

where $\$ \gamma_{\mu} p^{\mu}$, gives the spin density matrix for the virtual photon;

$$
\begin{aligned}
\rho^{\mu \nu} & =\frac{1}{-2 q^{2}} \operatorname{Tr}\left[\left(p+m_{e}\right) \gamma^{\mu}\left(p^{\prime}+m_{e}\right) \gamma^{\nu} \mid\right. \\
& \left.=\left(g^{\mu \nu}-\frac{q^{\mu} q^{\nu}}{q^{2}}\right)-\frac{(2 p-q)^{\mu}\left(2 p^{\prime}\right.}{q^{2}}-q\right)^{\nu}
\end{aligned}
$$

where $g^{\mu \nu}$ is the metric tensor for fat space-time wity trace -2 . The density matrix is not diagonal, so the photons are polarized aven though the electron beams are not.

Now, cunsider the full amplitude for the reaction of Fig. 1.1-b. The components are the two electron-phoion vertices, the fropagators of the viriual photons, and the amplitude for the coupling of two photons to the final state $X$. The latter is not specified at this point and simply is denoted by $M^{\mu \nu}$, so the expression for the full amplitude becomes

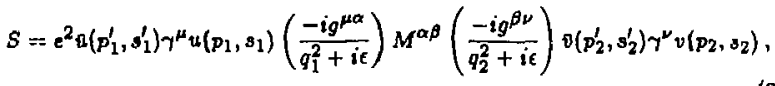

where the limit $\epsilon \rightarrow 0$ is to be taken. To form the cross section, this amplitude must be squared and sumn ₹d over all final states relevant to the experiment, averaged over all initial states, and multiplied by the incident fux. The various initial states are the spins of the unpolarized beam electrons, while the final state consists of a number of free particles, including the scattered beam electrons and the particles resulting from the $\gamma \gamma$ reaction. The latter heve continuous spectra of momenta, so the sum over final states becomes an integration, weighted by the density of final states, as well as a discrete sum over the spins. For the normalization we assume for plane-wave particle states, the density of final states for a single particle is $E_{i}^{-1}$, and the incident $\theta u x$ is $4\left\{\left(p_{1} p_{2}\right)^{2}-m_{1}^{2} m_{2}^{2}\right\}^{1 / 2} /(2 \pi)^{6}$, or to sufficient accuracy for an electron storage ring, simply $\mathcal{F}=4 E^{2} /(2 \pi)^{\circ}$. The factor of 4 comes from a sum over all possible electron polarization states. Derine $S_{f i}=\delta_{f i}+i \delta^{j}\left(P_{f}-P_{i}\right) T_{f i}$. Then the cross section for two-photon pariicle production at an $e^{+} e^{-}$storage ring is given by

$$
\begin{aligned}
\sigma & =\frac{1}{\xi} \frac{1}{V T} \sum_{f \neq i} s_{i j}^{\dagger} s_{f i} \\
& =\int \frac{(4 \pi \alpha)^{2}}{q_{1}^{2} q_{2}^{2}} \rho_{1}^{\mu \nu} \rho_{2}^{\alpha \beta} M^{\alpha \nu \beta} M^{\mu \alpha} \frac{(2 \pi)^{2} \delta\left(q_{1}+q_{2}-\sum_{i} k_{i}\right)}{4 E^{2}} \frac{\mathrm{d}^{3} p_{1}^{\prime} \mathrm{d}^{2} p_{2}^{\prime}}{E_{1}^{\prime} E_{2}^{\prime}} \prod_{j} \frac{\mathrm{d}^{3} k_{j}}{E_{j}},
\end{aligned}
$$

where $4 \pi \alpha \equiv e^{2}$

As is shown above, the invariant mass and momentum of the $\gamma \mathcal{\gamma}$ final state are determined by the momenta of the scattered beam electrons. Therefore, it is useful to differentiate Eqn. 2.10 in order to obtain the distribution of momenta of the scattered electrons. The resulting expression contains the tensor

$$
W^{\alpha \beta_{1} \mu \nu}=\frac{1}{2}(2 \pi)^{2} \int M^{* \alpha \beta} M^{\prime \nu} \delta\left(q_{1}+q_{2}-\sum_{i} k_{i}\right) \prod_{j} \frac{d^{3} k_{j}}{E_{j}}
$$

It must be true that $W^{\alpha \beta, \mu \nu}$ can be expanded into a sum of the various products of the available vectors, $q_{1}$ and $q_{2}$, and the metric tensor, $g^{\mu \nu}$. Budnev et oi. ${ }^{15}$ carry out the expansion in such a way that the result is explicitly Lorentz invariant, invariant under time reversal $(\alpha \beta+\mu \nu)$, and gauge in ariant $\left(g_{1}^{\mu} W^{\alpha \beta, \mu \nu}=\right.$ 
$q_{1}^{\alpha} W^{\alpha \beta, \mu \nu}=q_{2}^{\nu} W^{\alpha \beta, \mu \nu}=q_{2}^{\beta} W^{\alpha \beta, \mu \nu}=0$ ). When multiplied by the density matrices for the two virtual photons and summed over $\alpha \beta \mu \nu$, the differential cross section becomes ${ }^{16}$

$$
\begin{aligned}
\mathrm{d} \sigma=\frac{\alpha^{2}}{16 \pi^{4} q_{1}^{2} q_{2}^{2}} & \frac{\left[\left(q_{1} \cdot q_{2}\right)^{2}-q_{1}^{2} q_{2}^{2}\right]^{\frac{1}{2}}}{E^{2}}\left[4 \rho_{1}^{++} \rho_{2}^{++} \sigma_{T T}\right. \\
& +2\left|\rho_{1}^{+-} \rho_{2}^{+-}\right| \tau_{T T} \cos 2 \tilde{\varphi}+2 \rho_{1}^{++} \rho_{2}^{00} \sigma_{T L}+2 \rho_{1}^{00} \rho_{2}^{++} \sigma_{L T} \\
& \left.\quad+\rho_{1}^{00} \rho_{2}^{00} \sigma_{L L}-8\left|\rho_{1}^{+0} \rho_{2}^{+0}\right| \tau_{T L} \cos \tilde{\varphi}\right] \frac{\mathrm{d}^{3} p_{1}^{\prime} \mathrm{d}^{3} p_{2}^{\prime}}{E_{\mathrm{I}} E_{2}}
\end{aligned}
$$

where $\tilde{\varphi}$ is the angle between the scattering planes of the two beam electrons in the $\gamma \gamma$ center-of-mass system, and the $\sigma_{a b}$ are the cross sections for inteructions of longitudinal photons $(a, b \equiv L)$ and transverse photons $(a, b \equiv T) . \dagger$ The terms containing $\tau_{a b}$ are interference terms arising from polarization of the virtual photons. The quantities $\rho_{i}^{a b}$, with superscripts referring to the photon helicities, can be expressed as functions of only the scattered electron momenta, and they are given explicitly in equation 5.13 of Ref. 16. The one which is of special significance for untagged experiments is

$$
\rho_{1}^{++}=\frac{1}{2}\left[\frac{\left(2 p_{1} \cdot q_{2}-q_{1} \cdot q_{2}\right)^{2}}{\left(q_{1} \cdot q_{2}\right)^{2}-q_{1}^{2} q_{2}^{2}}+1+\frac{4 m_{e}^{2}}{q_{1}^{2}}\right] .
$$

Equation 2.12 shows that the $e^{+} e^{-} \rightarrow e^{+} e^{-} X$ cross section consists of the cross sections for $\gamma \gamma \rightarrow X$ convolved with known luminosity functions describing the production of the virtual photons. In the next section, we will see that under certain experimental conditions only $\sigma_{T T}$ is significant and the luminosity function factors into two parts-one describing each photon.

\footnotetext{
The term longitudinal photon refers to the component of the virtual-photon field with sero helicity (longitudinal polarisation of the electromagnetic field), while trangverse photon refero to the component with helicity $\pm I$ (trangverse polarization).
}

\subsection{The Equivalent Photon APPRoximation}

Because of the factor of $1 / q_{1}^{2} q_{2}^{2}$ in Eqn. 2.12, it is evident that the cross section is dominated by quasi-real (low $Q^{2}$ ) photons, which are associated with very small scattering angles of the beam electrons. If no requirement is made to tag the scaiterer electrons at finite angles, then the observed spectra must be dominated by,$\downarrow u s i-r e a l$ photons. In such a case it is advantageous to use an approximate form of the cross section.

Near mass shell, the cross sections for scattering of longitudinal photons varish, ${ }^{17}$ leaving only two terms in Eqn. 2.12. Since the scattered electrons are not tagged in this case, then the aximuthal angles are not known, resulting in an averaging over $\bar{\varphi}$. Therefore, the interference terms average to zero, and all polarization information is lost. Finalk, the approximation $q_{i}^{2} \ll W^{2}$ simplifies the density-matrix elements, resulting in a factorization of the cross section such that there is a separate factor for esch photon:

$$
\mathrm{d} \sigma=\sigma_{\gamma \gamma} \mathrm{d}^{2} n_{1} \mathrm{~d}^{2} n_{2},
$$

where $\sigma_{\gamma \gamma}$ is the cross section for scattering of two real, unpolarized shotons. This is equivalent to an expression for scattering of two beams of real phcitons with the flux of each given by

$$
\mathrm{d}^{2} n_{i}=\frac{\alpha}{\pi} \frac{\mathrm{d} x_{i}}{x_{i}} \frac{\mathrm{d} Q_{i}^{2}}{Q_{i}^{2}}\left[\left(1-x_{i}+\frac{1}{2} x_{i}^{2}\right)-\left(1-x_{i}\right) \frac{Q_{i \min }^{2}}{Q_{i}^{2}}\right]
$$

What we have arrived at is an equivalent photon approximation (EPA) for twophoton production, in which the electron beams have been replaced by equivalent fluxes of real photons.

Equations 2.14 and 2.15 are intuitively meaningful and are especially useful for doing simple calculations. However, it is important to keep in mind the approximations involved. First, all terms of order $Q^{2} / W^{2}$ in the expressions 
describing the kinematics have been neglected. Second, contributions frorn longitudinal photons have been neglected. For the case of lepton production, $\gamma \gamma \rightarrow l^{+} l^{-}$, the cross sections are known, and one finds that the ratios $\sigma_{L T} / \sigma_{T T}$ and $\sigma_{L L} / \sigma_{T T}$ are the order of $Q^{2} / W^{2}$. The third approximation is the neglect of the $Q^{2}$ dependence of the $\gamma 7$ cross sections. Again, for leplonic fial states the variation from the cross section at zero $Q^{2}$ is only the order of $Q^{2} / W^{2}$. The relevant cross sections are not known for the case of hadronic final states, but experience with hadronic form factors and $\gamma^{*} p$ collisions suggests that longitudinalphoton contributions and $Q^{2}$ variations become important only wher. $Q^{2}$ becomes the order of the square of the $\rho$ mass! 18

Therefore, EPA should be good as long as $Q^{2} \ll W^{2}$ and, for hadronic final states, $Q^{2} \ll m_{\rho}^{2}$. If the events are not tagged, then the vast majority of the observed events will satisfy these criteria. If effective antitagging is not available to restrict severely the accepted range of $Q^{2}$, then part of the observed cross section may not satisfy the criteria sufficiently well. This part will be small enough in most cases, relative to the peak at minimum $Q^{2}$, that distributions which are integrated over the range of $Q^{2}$ still will not be stro. .gly affected by the approximation.

In Appendix $A$ some techniques and examples are given on how to numerically integrate $\gamma \gamma$ cross sections with the EPA spectrum over a typical detector acceptance. And in Appendix B there is a discussion of how to do such integrations by Monte Carlo methods over an arbitrarily complicated detector acceptance. There the results from using the EPA spectrum are compared with a Monte Carlo integration of the first-order QED crose section made without any ' pproximations. One finds that for untagged events the results of the two calculations cannot be distinguished within the statistical precision of the DELCO data.

\subsection{LUMINOSITY FUNCTION FOR TAGGED EVENTS}

The equivalent photon approximation of Section 2.3 is expected to be of limited usefulness for tagged events because of the neglect of terms of order
$Q^{2} / W^{2}$. In DELCO, tagged events must have at least one photon with $Q^{2}$ greater than about $0.13 \mathrm{GeV}^{2}$, so with $W$ as low as $0.6 \mathrm{GeV}$, factors of $Q^{2} / W^{2}$ are not necessarily amall. Therefore, let us consider again the exact differential cross section of Eqn. 2.12. We are not concerned with double-tag events, in which both scattered electrons are observed, because the cross sections are too small to be useful in the present experiment. Therefore, the terms containing ${ }^{{ }^{T} T T} \cos 2 \dot{\varphi}$ or ${ }_{T} L \cos \bar{\varphi}$ both average to zero. AB for the rest of the terms, the $\rho_{i}^{a h}$ are known functions, but that is not so useful unless one also has detailed knowledge of $\sigma_{L T}$ and $\sigma_{L L}$ as well as $\sigma_{T T}$. Except for puraly QED processes, such knowledge is very limited.

One expects that for small angle tags, the $\sigma_{T T}$ term still dominates. Certainly, if one photon is antitagged, as in the DELCO experiment, then at least terms with $\sigma_{L L}$ still are heavily suppressed. Thus there are only two relevant luminosity functions, which can be related by $\mathcal{L}_{L T}=\varepsilon \mathcal{L}_{T T}$. Thi polarization parameter $\varepsilon$ may be expressed, for a single-tag experiment, as ${ }^{10}$

$$
\begin{aligned}
& \varepsilon=\frac{\rho_{2}^{00}}{2 \rho_{2}^{++}} \approx \frac{2(1-y)}{2-2 y+y^{2}}, \\
& y=\frac{q_{1} q_{2}}{p_{2} q_{1}}=1-\left(1-x_{2}\right) \cos ^{2}\left(\theta_{2} / 2\right)
\end{aligned}
$$

where electron number 2 is tagged at an angle $\theta_{2}$. For DELCO, $\cos ^{2}\left(\theta_{2} / 2\right) \sim 1$ for al! tagging angles, so $\varepsilon \approx 2\left(1-x_{2}\right) /\left(1-\left(1-x_{2}\right)^{2}\right]$, which is within experimental errors almost constant and unity over the kinematic region typically of interest. In practice, $\sigma_{L T}$ cannot usually be distinguished from $\sigma_{T T}$, so what one measures is $\sigma_{\text {exF }}=\sigma_{T T}+\varepsilon \sigma_{L T}$, for which the luminosity function simply is $\mathcal{L}_{T T}$. Even then, one russt have some way of parameterizing the $Q^{2}$ dependence of $\sigma_{\text {exp }}$, but that problem is defersed to the next section. Here we diseuss only how to calculate $\mathcal{L}_{T T}$ without making the assumption used in EPA that $Q^{2} / W^{2}$ is small for both photons. 
In Ref. 20 the caiculations of Bonneau et al. ${ }^{21}$ are used to derive a singletag luminowity function in which the $Q^{2}$ dependence of the untagged electron has been integrated out analytically, from the minimum given by Eqn. 2.3 up to the maximum of $4 E^{2}(1-x) \sin ^{2}(\hat{\theta} / 2)$, where $\hat{\theta}$ is the minimum tagging angle. As a result, Eqn. 2.12 is approximated by

$$
\frac{d \sigma}{\mathrm{d} x_{1} \mathrm{~d} x_{2} \mathrm{~d} \theta_{2}}=\frac{\mathrm{d} \mathcal{L}_{T T}}{\mathrm{~d} x_{1} \mathrm{~d} x_{2} \mathrm{~d} \theta_{2}} \sigma_{\exp }\left(k_{i}, Q_{2}^{2}\right)
$$

$\mathcal{L}_{T T}$ is the desired tagged luminosity function:

$$
\begin{aligned}
& \frac{\mathrm{d} \mathcal{L}_{T T}}{\mathrm{~d} x_{1} \mathrm{~d} x_{2} \mathrm{~d} \theta_{2}}=\frac{\alpha}{8 \pi^{2}} K \cot \frac{1}{2} \theta_{2}\left[\frac{\left(K-2 x_{1}\right)^{2}}{K^{2}}+1\right] \\
& \quad \times\left[\left(\frac{\left|K-2\left(x_{2}+\left(Q_{2} / 2 E\right)^{2}\right)\right|^{2}}{K^{2}}+1\right) \ln \left(\frac{2 E}{m} \frac{\left(1-x_{1}\right)}{x_{2}} \sin \frac{1}{2} \hat{\theta}\right)-2 \frac{1-x_{1}}{x_{1}^{2}}\right] .
\end{aligned}
$$

where $K \cong\left(W^{2}+Q_{2}^{2}\right) / 4 E^{2}$. Electron number 2 is arsumed $t=$ ho the one which is tagged.

It is difficult to estimate the effects of the approximations leading to Eqn, 2.18, but some idea of its validity can be obtained by comparing with the Vermaseren ${ }^{22}$ Monte Carlot calculation of $e^{+} e^{-} \rightarrow e^{+} e^{-} e^{+} e^{-}$. The Vermaseren program does an exact calculation of the matrix element for the two QED Feynman diagrams corresponding to Eqn. 2.12 and also includes four more diagrams which contribute to the same final state in leading order QED. Those four additional diagrams, however, play a minor role within the phase space covered by DELCO's acceptance.

This comparison has been made by using in Eqn. 2.17 a formula for $\sigma_{\gamma \uparrow \rightarrow e^{+} e^{-}}$ which really is completely valid only for $Q^{2}=0$. The acceptance is defined such that one of the final-state electrons scatters within $27 \mathrm{mrad}$ of the beam, another is in the tagging range $27<\theta<93 \mathrm{mrad}$, and the remaining two are within the central acceptance $-0.6<\cos \theta<0.6$. Also, the two electrons in the

Refer to Appendix B for a discuesion of how the Monte Carlo calculationa are done.

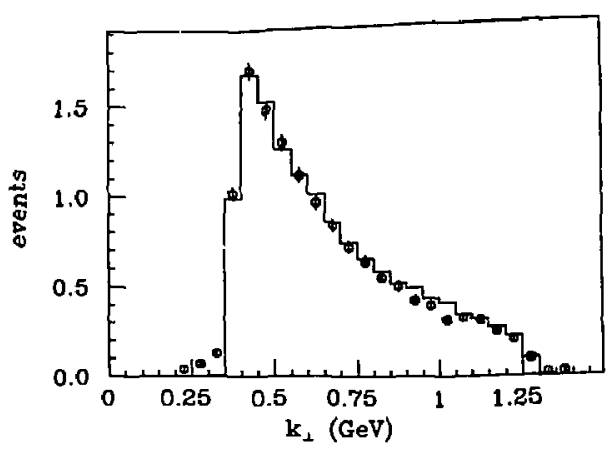

Figure 2.3. The distribution of $\boldsymbol{k}_{\perp}$ for tagged Monte Carlo events, comparing the simplified calculation (solid line) with that of the Vermaseren Monte Carlo. The two distributions are normalized to contain equal numbers of events total.

central acceptance must have an invariant mass in the range $0.6<W<2.6 \mathrm{GeV}$. Obviously, the approximation used for $\sigma_{T T}$ is not good since it contains no $Q^{2}$ dependence. Nonetheless, the shapes of the invariant-mass and angular distributions for the two electrons in the central acceptance are indistinguishable within the statistical precision available to the experiment. Even the distribution of the total trassverse momentum of the two electrons in the central acceptance agrees quite well, as seen in Fig. 2.3. However, the integrated cross section is about $10 \%$ higher thas that calculated by the Vermaseren program. Such a discrepancy is to be expected, since the $Q^{2}$ dependence of $\sigma_{T T}$ is neglected.

\subsection{Extrapolations of Cross Sections to LaRge $Q^{2}$}

Some simple models are available to predict the $Q^{2}$ behavior of cross sections in $\gamma \gamma \rightarrow$ hadrons. We consider just the case in which only one of the two photons has non-negligible $q^{2}$, so for example, $Q^{2}=-q_{2}^{2}$ and $q_{1}^{2} \approx 0$. The vector dominance 
model (VDM) predicts that for small $Q^{2}$, relative to $m_{\rho}^{2}$, for example, such cross sections are proportional to a $\rho$-pole form factor ${ }^{23}$

$$
F_{p}\left(Q^{2}\right)=\left(1+\frac{Q^{2}}{m_{p}^{2}}\right)^{-2}
$$

This model is extended in Ref. 24 to be valid for somewhat larger $Q^{2}$ by including contributions from the $\omega$ and $\phi$ vector mesons plus a term designed to approximate the higher resonances and the continuum. Also, for large $Q^{2}$ it is important to include effects from longitudinally polarized photons. That is done by assuming terms for $\sigma_{L T}$ identical to those for $\sigma_{T T}$, only suppressed by a factor of $Q^{2} / 4 m_{V}^{2}$, where $m_{V}$ is the relevant vector meson mass. Overall, the prediction for the measured cross section is given by a generalized vector dominance model (GVDM)

$$
\begin{aligned}
\sigma_{\text {exp }} & =\sigma_{T T}+E \sigma_{L T} \\
& =\left[\sum_{V=\rho, \omega, \phi} r V\left(1+\frac{Q^{2}}{4 m_{V}^{2}}\right)\left(1+\frac{Q^{2}}{m_{V}^{2}}\right)^{-2}+r_{c}\left(1+\frac{Q^{2}}{m_{0}^{2}}\right)^{-1}\right] \cdot \sigma_{\gamma \gamma}\left(Q^{2}=0\right) .
\end{aligned}
$$

The five parameters are determined by fitting to lepton-nucleon scattering data, for which this model does give a good fit. The numbers given in Ref, 24 are $r_{p}=0.65, r_{\omega}=0.08, r_{\phi}=0.05, r_{c}=0.22$, and $m_{0}=1.4 \mathrm{GeV}$.

The GVDM prediction may be used to extrapolate the measured two-photon partial widths of the $f$ and $f^{\prime}$ to $Q^{2}=0$. However, a Monte Carlo calculation of the model for $\gamma \gamma \rightarrow \pi^{+} \pi^{-}$described in Chapter 7 reveals that the cross section integrated over the acceptance for an untagged experiment decreases by only $1.4 \%$ when the form factor of Eqn. 2.19 is introduced, which almost certainly is an overestimate of the effect. Such a correction is less than the typical experimental uncertainty and thus is not critical. It is of interest, however, to compare the prediction of Eqn. 2.20 with the ratio of results from untagged and tagged experiments.

\section{Description of the Apparatus}

The DELCO experiment is a collaborative effort of groups from California Institute of Technology, Stanford University, and Stanford Linear Accelerator Center (SLAC). The DELCO detector accumulated data in one of the six interaction regions of the $2.5 \mathrm{~km}$ circumference $P E P$ electron-positron colliding beam storage ring for a period of threc years. $\dagger$ The beam energy was $14.5 \mathrm{GeV}$, and the luminosity ranged from about $10^{30}$ to $10^{31} \mathrm{~cm}^{-2} \mathrm{~s}^{-1}$, giving a total luminosity integrated over the running time of about $170 \mathrm{pb}^{-1}$.

Figure 3.1 and Fig. 3.2 show longitudinal and transverse cross sections of the detector. The most important detector components are designed to intercept particlea which acatter at large angles $\left(>45^{\circ}\right)$ from the beam line. Travelling outward from the interaction point, such a particle first passes through the wall of the vacuum pipe and into the inner tracking chambers. Just outside of those are Cerenkov counters, followed by additional tracking chambers, a system of time-offlight counters, and a shower counter system.

The reference coordinates used to describe the experiment assume that the positive $z$ axis is along the beam line in the direction of the positrons (southward). The positive $y$ axis points upward, and the positive $x$ axis points toward the center of the storage ring. The origin is at the detector center, where the beams collide.

\subsection{TRACKING SYSTEM}

The inner tracking system consists of two concentric cylindrical drift chambers placed between the poles of a dipole magnet. The magnet produces a nonumiform field in the tracking volume. Its coil are operated with a 3000 ampere current, which saturates the steel pole pieces at a level of about 20 kilogauss. The resulting field at the interaction point is 3.3 kilogauss, and the integral $\int \vec{B} \times d \vec{l}$ for a particle produced at the center and traveling outward to infinity is 1.8 kilogauss-meters.

$\dagger$ The running period was divided into three time blocks, which we denote by 1982, 1989, and 198. Aetually, each time block includes the Autumn running period of the previous calendar year. 


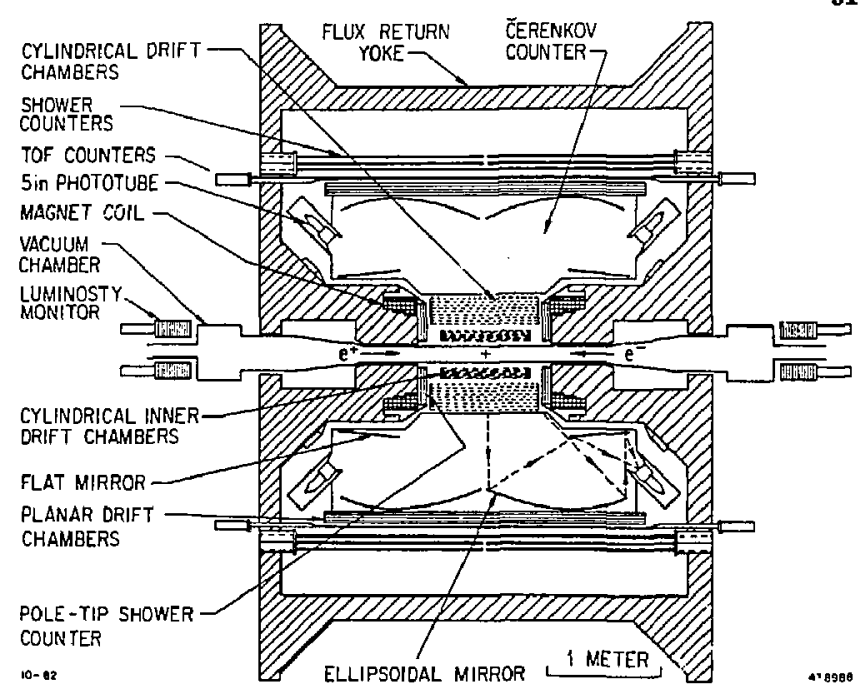

Figure 3.1. Longitudinal cross section of the DELCO detector.

The drift chambers produce a maximum of 16 two-dimensional position measurements for each track. Eight are from layers of cells, called $Z$ layers, arranged parallel to the beam axis, which measure only the coordinates in the transverse $x-y$ plane. Eight more layers consist of stereo cells, arranged with a transverse angle of $\pm 3^{\circ}$ with respect to the $Z$ cells. They are called $U$ or $V$ layers, depending on the sign of the stereo angle. When used in conjunction with the $Z$ layers, they measure the angle out of the transverse plane (the dip angle $\lambda$ ), as well as giving additional information on the $x-y$ coordinates. The arrangement of the 16 layers is UUZZVVZ ZUUZZVVZZ. The total number of cells is 1214.

Each cell is rectangular in cross section, with boundaries defined by $120 \mu$

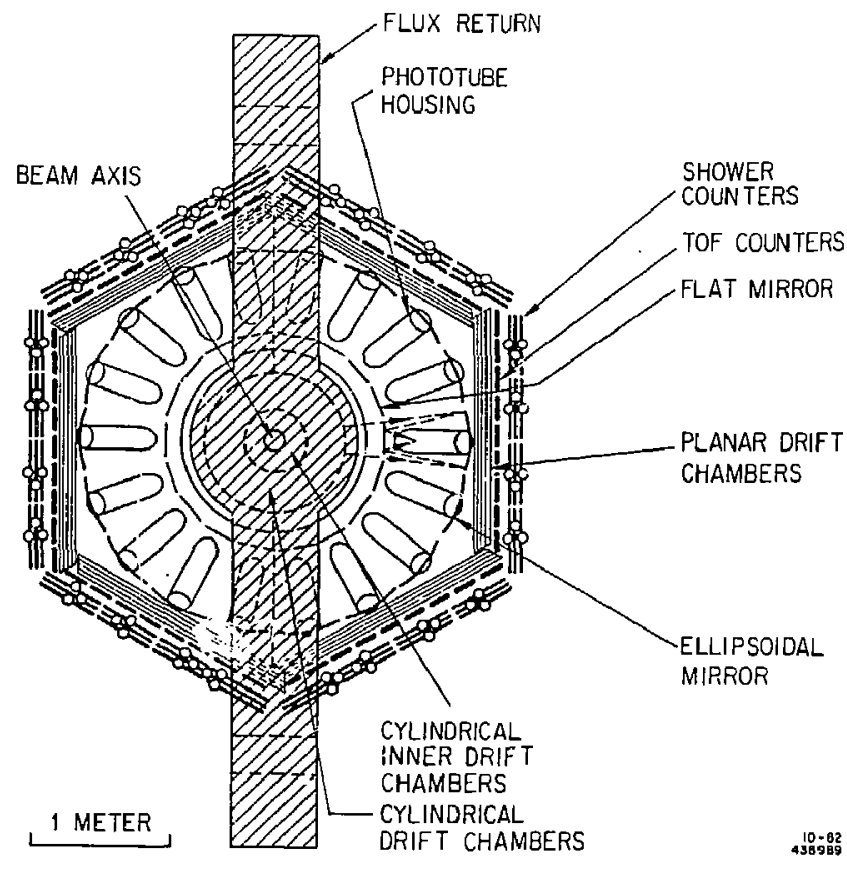

Figure 3.2. Transverse cross section of the DELCO detector. 
diameter field wires and guard wires at high voltage. At the center is a thin (30 or $38 \mu \mathrm{m}$ ) gold plated sense wire at ground potential. Figure 3.3a shows a crosa section of a typical cell. The drift chambers are filled with gas mixtures only slightly above atmospheric pressure. For the first year of running, the mixture was $55 \%$ argon and $45 \%$ ethane, and thereafter a mixture of $89 \%$ argon, $10 \%$ carbon dioxide, and $1 \%$ methane was used.

A particle travelling radially outward leaves clusters of ionization in the cells along ite path. Those which are not too close to the cell boundary drift toward the sense wire along the electric field with a constant velocity of about $5 \mathrm{~cm} / \mu \mathrm{sec}$. The field intensity increases as the wire nears, so close to the thin sense wire an avalanche occurs, giving a gas gain of the order of $10^{7}$. The time of the resulting signal is measured with respect to the beam crossing time to give the drift time. That is converted to a distance by a nonlinear relation determined by an iterative fit to actual data. Whether the track is to the right or the left of the sense wire by that distance must be determined from a global fit of all the individual measurements of a single track. The resulting single-cell resolution varies from layer to layer between $150 \mu \mathrm{m}$ and $250 \mu \mathrm{m}$.

The outer tracking system consists of six planes of six layers each of drift chambers arranged in a hexagon about the Cerenkov counters. Four of the plenes have cells parallel to the beam line, and two have large-angle $\left(30^{\circ}\right)$ stereo cells. Each cell is rectangular, as shown in Fig. 3.36 , with a solid aluminum perimeter. Typical operating voltages are indicated in the figure. The maximum drift distance of $4 \mathrm{~cm}$ is much longer than that of the inner chambers. The single-cell resolution is on average about $500 \mu \mathrm{m}$.

Tracking measurements in the $x-y$ plane give the curvature $x$ of a track, and that combined with the dip angle $\lambda$ gives the momentum. The full set of parameters used to describe a track are $\kappa$ and $\tan \lambda$, along with $\phi_{0}, z_{0}$, and $x_{t}$; respectively the $\phi$ direction, $z$ position, and distance from the beam line at the point of closest approach of the track and beam. A pattern recognition program
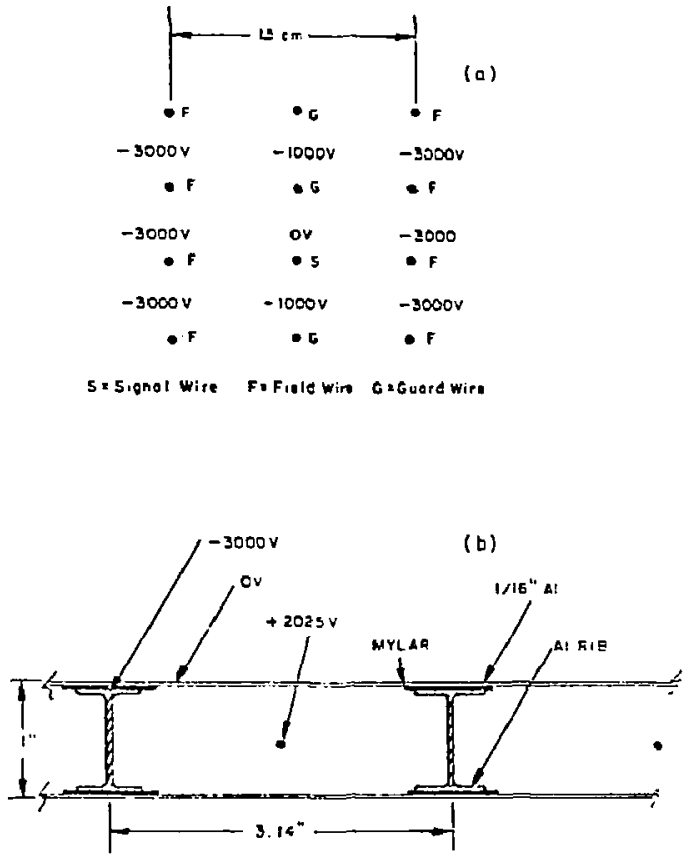

Figure 3.3. Drift chamber cell configurations. (a) A typical cell of the inner cylindrical drift chambers. (b) A cell of the outer planar drift chambers. 
associates the individual measurements, called hits, to form a track and does a preliminary fit of these parameters. A track fitting program then does a detailed fit of each track using all the information known about the nonuniform magnetic field. For the events considered in this thesis, this procedure is relatively uncomplicated and error free, because the tracks, being opposite each other, never interfere with one another.

For electrons from Bhabla scattering, the tracking system messures the momenta with a resolution of $\sigma_{p}, p=0.02 \cdot p$, where $p$ is in units of GeV. For measuring such high momentum ( $p=14.5 \mathrm{GeV}$ ) tracks, the outer drift chambers are essential, since even at that radius the total track deflection from the magnetic field is only about $2 \mathrm{~mm}$. The errors come from single-cell time measurements and from survey measurements of the positions of the outer drift chambers. The opposite is true for two-photon events, where $0.5 \mathrm{GeV}$ is a typical momentum. For them, the measurement error is dominated by multiple scattering of particles in the detector material. Due to the relatively large amount of material preceding and within the outer drift chambers, those chambers are not useful for momentum measurement in two-photon events. Consequently, their measurements are heavily deweighted in the tracking fit. In fact, no significant change in resolution is observed when they are removed from the fit entirely. They are useful for projecting the track into the time-of-fight and shower counter systems, which lie immediately beyond them.

Since the outer drift chambers do not contribute to momentum measurements, the material which causes the multiple scattering limitation to the momentum resolution is that located between the beampipe vacuum and the drift chamber gas, plus the thin wall between the two inner drift chambers. Additional small contributions come from the gas and wires within the tracking volume. The thickness of the beam pipe plus the adjacent inner drift chamber wall was 0.029 radiation lengths of aluminum for the $1982 / 1989$ running period. Before the 1984 run the beampipe was replaced, reducing the thickness to 0.013 radjation lengths?
The wall between the two inner drift ehambers was 0.005 radiation lengths thick.

The detector simulation program calculates multiple scattering of individual particies according to a simple gaussian distribution. The angle of deflection in three dimensional space is distributed according to

$$
P(\phi, \theta) \mathrm{d} \theta \mathrm{d} \phi=\frac{1}{2 \pi \theta_{0}^{2}} e^{-\theta^{2} / 2 \theta_{0}^{2}} \sin \theta \mathrm{d} \theta \mathrm{d} \phi
$$

where $\theta_{0}$ depends on the momentum and the material according to ${ }^{26}$

$$
\theta_{0}=\frac{14.1 \mathrm{MeV}}{{ }^{\beta} \beta} Z_{\mathrm{inc} .} \sqrt{L / L_{R}}\left[1+\frac{1}{9} \log _{10}\left(L / L_{R}\right)\right] \text { (radians) }
$$

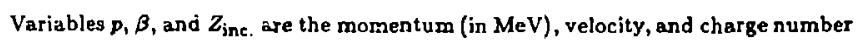
of the incident particie, and $L / L_{R}$ is the thickness in radiation lengths of the layer of material through which the particle passes. Equation 3.1 is an accurate approximation to the body of the true distribution, but it larks the small and slowly falling tails which are caused by single hard scattering effects. In the DELCO programs, the material itself is represented by simple concentric cylinders with thin walls. The gas and wires are approximated by the equivalent average amount of material and are treated in the same way as detector boundaries.

Measurement errors for single cells are approximated by smearing the expected drift times according to gaussion distributions of the appropriate width. Bhabha scattering events are used to determine the widths, which works well because the $14.5 \mathrm{GeV}$ tracks suffer very little from multiple scattering.

Such a Monte Carlu jimulation of the process $e^{+} e^{-} \rightarrow e^{+} e^{-} \mu^{+} \mu^{-}$predicts the resolutions given in Table 3.1 for the momentum measurement of the muons. These predictions are checked against the data by considering the distribution of the quantity $k_{\perp}=\left|\vec{p}_{\perp}^{\mu_{1}}+\vec{p}_{\perp}^{\mu_{2}}\right|$, which is sharply peaked toward $k_{\perp}=0$ and thus highly sensitive to measurement errors. One finds poor agreement; the Monte Carlo prediction is more sharply peaked (smeared less) than the data. If instead 
of uning the detailed detector simulation, the Monte Carlo generated variables $\phi$, $\tan \lambda$, and $\kappa$ simply are smeared by gaussian distributions of the widths given in Table 3.1, then the same discrepsicy is found. In fact, one finds that, for the $1988 / 1989$ data, it is necessary to increase $\sigma_{\kappa} / \kappa$ to 0.08 , with $\sigma_{\phi}$ and $\sigma_{\tan \lambda}$ fixed, in order to achieve good agreement with the data. Various factors besides the momentum resolution, such as radiative effects and the intrinsic $p_{\perp}$ of the focussed colliding electron beams, have been cheiked to be sure that they cannot account for the discrepancy, so the problem does seem to lie with the momentum resolution.

Table 3.1. Monte Carlo predictions for the momentum resolution of muons from the process $e^{+} e^{-} \rightarrow e^{+} e^{-} \mu^{+} \mu^{-}$

\begin{tabular}{|c|l|l|}
\hline & \multicolumn{1}{|c|}{$1989 / 1989$} & \multicolumn{1}{|c|}{1984} \\
\hline$\sigma_{\phi}$ & 0.0058 radian & 0.0043 radian \\
$\sigma_{\tan \lambda}$ & 0.0077 & 0.0060 \\
$\sigma_{\kappa} / \kappa$ & 0.056 & 0.053 \\
\hline
\end{tabular}

A useful process for checking the momentum resolution is $K_{*} \rightarrow \pi^{+} \pi^{-}$. A clean sample of 188 such decays, in which the two pion tracks are well separated from any others in the event, were found in the $1982 / 1989$ data by searching for the characteristic decay vertex. The resulting $\pi^{+} \pi^{-}$mass peak. along with a gaussian fit, is shown by Fig. 3.4. The width of the fitted gaussian is $\sigma_{m}=19.6 \pm 0.9 \mathrm{MeV}$. Generating similar decays by Monte Carlo, including the detailed detector simulation, yields a peak with $\sigma_{m}=18.2 \pm 0.5 \mathrm{MeV}$. And when the Monte Carlo resolution $\sigma_{\kappa}$ is increased enough to give good agreement with the $k_{\perp}$ distribution from two-photon pair production, then the $K_{\mathrm{s}}$ width increases to $21.3 \pm 0.7 \mathrm{MeV}$. Thus the width from data falls about talfway hetween the two Monte Carlo assumptions. Unfortunately, there are not enough $K$, to make the result highly significant. We conclude that, for low-momentum tracks.

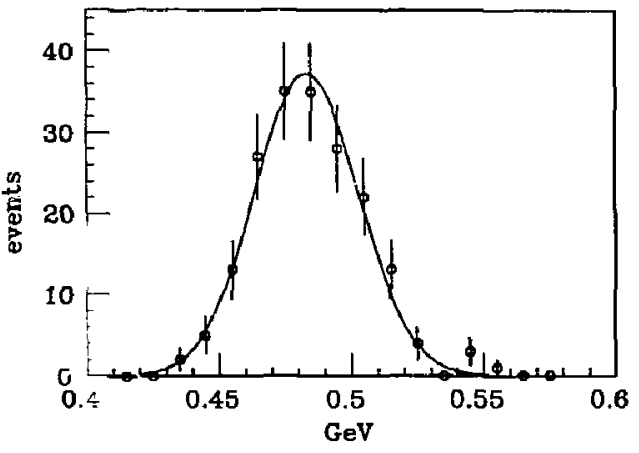

Figure 3.4. The mieasured $K$, mass distribution for $1888 / 1989$ data. The solid curve is a geissian fit.

$\sigma_{\kappa} / \kappa=0.07 \pm 0.01$. This result also is adequate for the 1981 data. Figure 3.5 compares the $k_{\perp}$ distribution from two-photon produced electron pairs with Monte Carlo predictions for a zaussian resolution of $a_{\kappa} / \kappa=0.07$. The angular resolutions are fixed as specified in Table 3.1. It is clear that a gaussian resolution function does not reproduce the shape of the observed $k_{\perp}$ distribution very well. However, none of the other kinematic distributions of interest are nearly as sensitive to the momentum resolutior The uncertainty of $\pm 1 \%$ in the momentum resolution is taken into account $w$ t $n$ determining the gystematic errors in the analyses which follow. With the assumption $\sigma_{\kappa} / \kappa=0.07$, the resolution for the invariant mass $W$ of a pion pair in the $f$ iesonance region $(0.9<W<1.4 \mathrm{G} \mathrm{VV})$ is $\sigma_{W}=0.053 \mathrm{GeV}$.

Finally, one can see from Fig. 3.4 that the $K_{a}$ peak is as much as $2 \%$ low. Measurements of the DELCO magnetic field, however, indicate that the energy scale of the experiment should be accurate to within $\pm 1 \%$. In any case, the analysis in this thesis is done in such a way that it is insensitive to any such systematic shifts of the overall energy scale. 


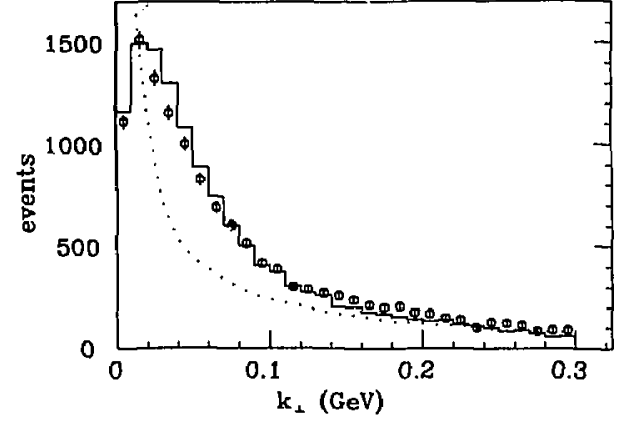

Figure 3.5. The measured distribution of $k_{\perp}$. The points show 1982/1989 data, which is compared with a Monte Carlo prediction assuming a momentum resolution of $\sigma_{\kappa} / \kappa=0.07$. The smooth dotted curve shows the Monte Carlo distribution before adding resolution effects.

\section{2 ČERENKOV COUNTER SYSTEM}

The DELCO detector was designed to accommodate a large system of highly efficient gas threshold Cerenkov counters with a minimal amount of material between the beam-beam interaction point and the Cerenkov radiator. This dictates, for example, the config'ıration of the magnetic field, with the coils placed

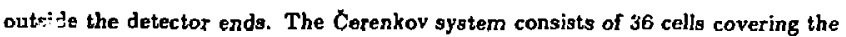
regicn $\pi / 4<\theta<3 \pi / 4$ and $0<\phi<2 \pi$, which is about $70 \%$ of the full solid angle. There are 18 divisions of the azimuthal angle, and the polar acceptance is divided about the $z=0$ midplane of the detector.

Two separate data sets vere accumulated by the DELCO experiment. In one, the Cerenkov system was filled with atmospheric pressure isobutane. In the other, it was filled with atmospheric pressure nitrogen, which is less dense and therefore gives higher particle thresholds. The radiator path lengths range from 55 to $110 \mathrm{~cm}$. For the isobutane radiator and an incident high momentum electron, this results in an average of 30 photoelectrons produced at the light detecting photomultiplier tubes. Consequently the efficiency is almost $100 \%$ throughout the active volume. Section 4.4 discusses in some detail this question of efficiency as related to the analysis at hand.

The counter optical system is designed for efficient collection of light produced by straight tracks from the origin. That means that if the particle is only slightly above threshold, so that the light is emitted in a cone of large angle about the track, or if the track is at such a low momentum that its curvature in the magnetic ficld is significant, then the light collection efficiency suffers somewhat. Figure 3.6 is a drawing of the optical system. Large glass elliptical mirrors, one for each cell, are aituated such that one focus is at the interaction point and the other is at the face of the phototube. Therefore, all pathe from the interaction point to a phototube are of the same length. Winston cones ${ }^{27}$ placed before the phototubes aid in the collection of light which is not focussed directly ort to the photocathode. Each phototube is protected from the fringe field of the magnetic spectrometer by a small compensating coil and four concentric cylinders of ferromagnetic material.

Both the pulse heights and times of signals from the phototubes are digitized. The aystem is calibrated by using electrons from the processes $e^{+} e^{-} \rightarrow e^{+} e^{-}$and $e^{+} e^{-} \rightarrow e^{+} e^{-} e^{+} e^{-}$. The path length of each track through the counter is known from tracking measurements. The calibration procedure is designed to correct the observed pulse height associated with a given track for the track's path length and the collection efficiency of the particular cell. The resulting corrected pulse heights are normalized to a path length of $100 \mathrm{~cm}$. The time corrections are adjusted suc' that the mean time is zero. See Ref. 28 for a complete description of the counter design, construction, calibration, and performance.

\subsection{Barret SHower COUNTERS}

The barsel shower counters consist of six sextants arranged in a hexagon surrounding the other detector components. Each sextant contains four longitudinal 


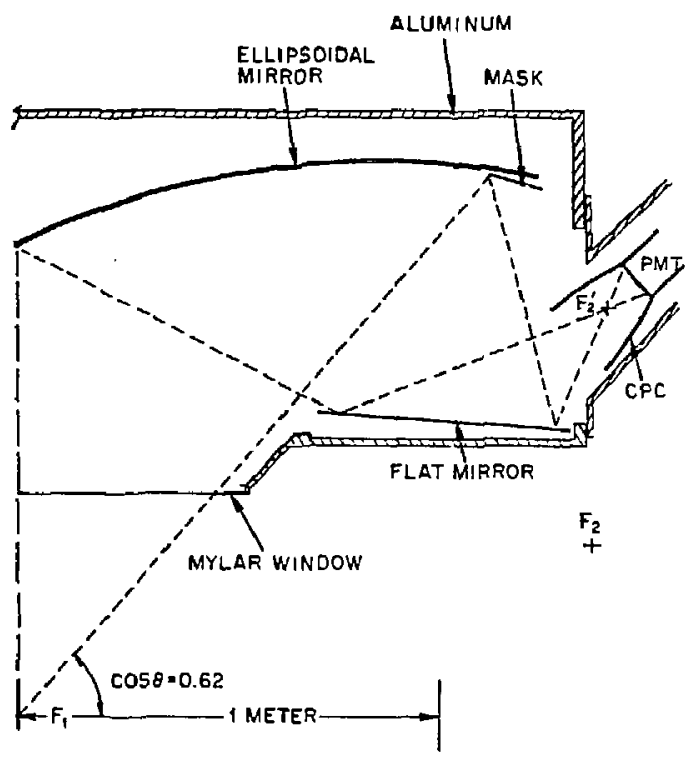

Figure 3.6. The optical aystem of a Cerenkov counter cell.

courter modules, each with three $1 / 2$ inch thick layers of lead interleaved with three $1 / 4$ inch layers of acrylic scin tillator. An aluminum structure supports the four modules with a $1 / 4$ inch thick plane in fronk facing the interaction point, a 1 inch plane in back, and $1 / 4$ inch thick planes on the sides and between adjacent modules. The first scintillator of each module is read out by phototubes at both ends, while each of the second and third layers are separated by mirrors at $z=0$ into two counters which are read out at opposite ends of the detector. The total amount of material preceding the last layer of scintillator is 6.9 radiation lengths.

The counter system may be used to distinguish electrons from minimum ionizing particles, especially at high momenta. However, they are not used for that purpose in the analysis of two-photon events. Such events are low in energy and have widely spaced tracks, which allows identification of electrons by the Cerenkov systern. The only purpose of the shower counters in this analysis is to reject background from two-track events with additional particles not detected by the traciking system. Layers 7 through 16 of the inner tracking system have a small dead region at the $y-z$ (vertical) plane which can allow some untracked charged particles into the barrel shower counter region. But for the most part the tracking coverage is more complete than the barrel shower counter coverage, so the main objective of the shower counters is to veto events with photons in the final state.

The barrel shower counters cover the range in pols: agle from bout $\cos \theta=-0.6$ to $\cos \theta=0.6$ (the exact cutoff depends on $\phi$, aince the counters are planar). The aximuthal coverage is broken by $3 / 4$ inch gaps between counters and larger gaps between sextants, so the total solid angle covered is only $0.52 \cdot 4 \pi$ steradians. Thus it is possible for many photons to escape detection. However, such background can be estimated, and as we will see, it is not a serious problem.

The only other use of the barrel shower counters which is relevant to this analysis is in the detector trigger. In fact, all of the useful triggers have a shower counter component. That is unfortunate, for such a trigger is inefficient in the detection of low energy, low multiplicity events, and there is little trigger redundancy to sploit in understanding the trigger efficiency. Consequently, the most difficult and most important part of the analysis is to understand the efficiency of the shower counter system.

The individual counters are said to latch when the phototube pulse height is above a low threshold near the beam crossing time. The threshold is a small fraction of the signal expected from a single ionizing particle, so one finds no noticeable inefficiency of the scintillation counters themselves. However, to produce a shower counter latch, the trigger logic requires two out of three of the layers in a single module to latch. Therefore, particles must pass through all 
of the detector material between the interaction point and the second layer of scintillator before a shower counter latch can occur. That is a total of about 5 radiation lengths or 0.3 interaction lengths.

In later chapters the shower counter latch efficiency is determined for electrons and pions from the data by exploiting what trigger redundancy there is. However, it is important to check the results by calculation. That is done by using the Electron-Garama-Shower (EGS) Monte Carlo ${ }^{20}$ and the High Energy Transport Code (HETC) Monte Carlo ${ }^{30}$ to predict the fractions of electrons and pions which will penetrate, in some form, through the material. Special code has been written for this analysis to interface these programs with the detailed geometry of the DELCO barrel shower counters, plus the layers of aluminum, scintillator and glass which precede the shower counters. That in turn is interfaced with the rest of the detector aimulation code for the tracking chambers and the Cerenkov counters.

\subsection{POLETIP SHOWER COUNTERS}

The magnet pole tips are covered by another set of lead-scintillator shower counters consisting of $0.53 \mathrm{~cm}$ thick layers of lead interleaved with $1 / 4$ inch thick acrylic scintillator. The total thickness is 5 radiation lengths. Each poletip counter is divided into 18 arimuthal wedge-shaped segments, each of which is read out by BBQ wavelength shifter and a single phototube. There is no radial segmentation. The counters cover the angular range $0.81<|\cos \theta|<0.98$, which is $17 \%$ of the total solid angle.

In this analysis, these counters are used only as veto counters. No events are accepted which deposit energy above noise level in the poletip counters, whether the energy is from charged particles or photons. Combining the poletip counters with the barrel shower counters gives a total coverage of $0.69 \cdot 4 \pi$ steradians for vetoing events with photons and extraneous charged particles.

\subsection{Time of Flight Counters}

A set of 521 -inch thick by 8 inches wide acrylic scintillation counters are mounted on the face of the barrel shower counters with 8 or 9 per sextant. Each is read out at both ends by phototubes. They are used in this analysis to reject cosmic ray background (see Section 5.2 ) and to identify kaons and protons by time-of-flight measurement. The average time resolution for minimum ionizing particles is roughly $330 \mathrm{ps}$. Refer to Chajer 9 for a detailed discussion of their use and performance in particle identification.

\subsection{LUMINOSITY MONITOR}

The luminosity monitor is made of two sets of gix lead-scintillator shower counters, with one at each end of the detector about $280 \mathrm{~cm}$ from the interaction point. Each set forms a hexagon about the bearn pipe and is segmented into sextants. Each sextant is composed of 16 sheets of $0.56 \mathrm{~cm}$ thick lead interleaved with $1 / 4$ inch thick scintillator. All sixteen layers of a sextant are read out by a single phototube by way of a BBQ wavelength shifter bar. In addition there is a scintillator on the front of each sextant which is read out separately. The angular range covered by the counters is roughly $25-90$ milliradians with respect to the beam axis.

The counters are designed to monitor beam luminosity by measuring the rate of Bhabha scattering. In this analysis they are used to determine the relative integrated luminosity of one run with respect to another, but no attempt is made to obtain from them an absolute normalization. The Bhabha scattering cross section varies like $1 / \theta^{4}$ at these small angles, which necessitates a very accurate knowledge of the counter acceptance in order to predict the integral of the cross suction over the acceptance with sufficient accuracy to be used as an absolute normalization. It turns out that it is easier to normalize to measurements of QED processes in the central detector. 
An important use of the luminosity counters in this analysis is to tag (or antitag) two-photon events by detecting (or vetoing) the scattered beam electrons. They are not used to help veto events with photons in the final state because of the relatively large background of synchrotron radiation from tise beams at those small angles. Beam electrons scattered with energies around $14 \mathrm{GeV}$, however, are well separated from the noise and are relatively easy to detect. $\dagger$ We defer further discussion of the tag analysis to Section 4.9.

\subsection{EVENT TRIGGER}

Signals from the various detector systems are processed by on-line hardware immediately after each beam crossing in order to determine whether anything interesting occurred and the data should be written to tape. This trigger process occurs in two stages. The first stage considers only information fr 3 m the various counters and is fast enough to make the necessary decision between beam crossings (every $2.4 \mu \mathrm{sec}$ ). There are several logical combinations of counters which can produce auch a level-1 trigger, and for a high-energy, high-multiplicity event most of them are redundant. For detecting pair production by two photons, however, only three triggers are relevant, and there is not always a redundancy.

Each of the three triggers, which we denote by $K S, 2 S$, and $L S$, includes a barrel shower counter requirement. The KS trigger requires at least one detector sextant to have one or more srenkov counters fired in coincidence with one or more shower counter latches. Recall that a shower counter latch is defined to be a coincidence of at least two layers of a single module. The $2 \mathrm{~S}$ trigger requires at least two different sextants to have one or more shower counter latches, and the LS trigger requires that at least one of the two luminosity counters fire in coincidence with one or more shower counter latches. For all of these triggers, the coincidence time window is centered about the beam crossing time and is sufficiently large to accept all beam related events.

$\uparrow$ Such tagging cannot bo done with the polatip counters, however, which are too thin to distinguish reliably between such high momentum electrons and photons at much lower energies.
The second stage of trigger processing considers the tracking information from the inner drift chambers and commences only if an event aatisfies the level-1 trigger. There also is a level-2 trigger which depends only upon "neutraln energy, but it is not relevant for this analysis. We do not discuss in detail how the hardware defines a "track" but only mention that the definition can accommodate true tracks with momenta as low as $0.1 \mathrm{GeV}$. The level-2 requirement is for at least one "track" to be found in the inner drift chambers. For the 1981 data, there is also the additional requirement that it must point roughly toward a fired shower counter. If an event satisfies the level-2 requirement, then it is read into the computer and written to tape. The rate of events satisfying the level-2 tracking requirement was typically 1-2 hertz.

The level-2 trigger introduces no loss of efficiency for event types of interest in this analyais-any event leaving two tracks roughly back-to-back in $\phi$, through enough cells such that they could be found by the full pattern recognition program, certainly will fulfil this part of the trigger requirement. That is verified by the fact that all such two-track events have two or more "tracks" found by the hardware processor.

The level-1 trigger is more of a problem. Aside from the obvious geometric inefficiencies of the various trigger components, the only significant ineficiency which has been found is caused by actual absorption of particles by the material of the detector. That of course depends on the type of particle involved, so we defer all further discussion of trigger efficiency to the sections in which the reaction channels of interest are studied one by one. For a more complete discussion of the hardware of the detector trigger and data acquisition system, one may refer to Ref. 31 . 
3.8 Data Processing and Reduction

\subsubsection{Pass-1 Filter}

The first step in off-line processing of data is to analyze all events with a set of programs called the Pass-1 filter. This filter analyzes all the detector systems, starting with the most simple, and rejects after as little computation as possible those events which cannot possibly be of any use for physics. The cuts made generally cause no inefficiency for physics data beyond what is inherent in the trigger and the detector systems themselves. In this section, only those processing steps which are important to an understanding of the analysis in this thesis are considered.

The first stage of processing considers the information from the shower counters and time-of-fight counters. There must be at least one counter of either system measuring a time-of-fight of less than $11 \mathrm{~ns}$ and greater than 3 ns. Such a measurement requires a counter with valid time measurements at both ends, but that much is almost certain for events satisfying one or more of the triggers, all of which require shower counter latches. Furthermore, in order to be so identified, kaon pairs and proton pairs must have good timing information from the timeof-fight counters for both tracks. The only potential inefficiency, then, is from the 11 ns cut itself. However, it turns out that kaon pairs and proton pairs with enough energy to satisfy the $2 \mathrm{~S}$ trigger have times of flight to the counter systems which are short enough not to be affected by this cut.

The next stage of processing considers the hits in the tracking chambers in a simple manner similar to what already has been done by the hardware processor of the trigger. The objective is to eliminate quickly events with not enough drift chamber hits to form at least two separate tracks and to eliminate events caused by large bursts of noise. These cuts introduce no inefficiency.

The following stage involves the detailed pattern recognition necessary to associate drift chamber hits into complete tracks, each with measured curvature and direction. It is important that the preceding stages eliminate as many junk

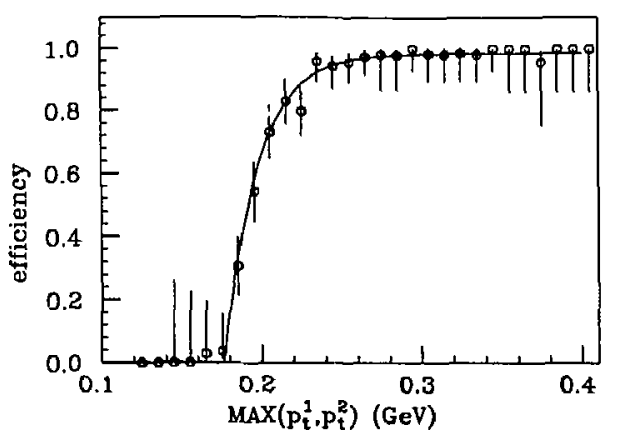

Figure 3.7. The efficiency of the pattern secognition early abort feature used in the Pass-1 filter for two-track events.

events as possible, because the pattern recognition is relatively time consuming. For the same reason, the pattern recognition programs are design to abort early those events which do not look promising. The programs look first for relatively straight tracks coming from close to the origin and having associated hits from both the inner and outer tracking systems. If no such tracks are found in the first pass, then the event is rejected. The result is that events in which no trar': sas a transverse momentum preater than about $0.2 \mathrm{GeV}$ get rejected. Actually, the cutoff is somewhat soft, as shown by Fig. 3.7.

The Pass-1 filter requires that each event have at least two tracks found by pattern recognition. Details of the pattern recognition are not discussed here, because although the procedure is complicated in general, for events with only two tracks it is relatively simple and not problematic. The only problem which occasionally ariaes is when a large amount of noise overlaps a track or mimics a track.

After pattern recognition is complete, the resulting tracks are extrapolated in order to associate individual Cerenkov, time-of-fight, and shower counters with 
each track. At this point, the Pass-1 filter requires that at least one track have in in-time time-of-flight or barrel shower counter associated with it in this manner.

\subsubsection{Event Classification}

After the Pass-1 filter has been applied, the results are passed through several filters designed to classify and separate the events into several physics and calibration categories. The calegories are not exclusive, and the cuts applied generally are very loose. By far the largest physics data set is generated by the two-photon filter, which is the only one of interest here.

All of the cuts of the two-photon filter are superseded by analysis cuts discussed in the following chapters. First, very obvious cosmic rays aro rejected, but the cosmic-ray cut discussed in Section 5.2 is more severe. Second, all events with greater than $4 \mathrm{GeV}$ of energy detected in the luminosity counters are accepted as tagged event candidates. The remaining events are rubjected to four additional loose cuts, designed to accept charged exclusive events: The total event charge must be no more than 2 in absolute value; the average of the $z$ coordinates of the track origine must be less than $8 \mathrm{~cm}$ in absolute value; the sum of the track momenta must be less than $5.5 \mathrm{GeV}$; and the total transverse momen tum $k_{\perp}$ must be less than $0.6 \mathrm{GeV}$,

\subsubsection{Track Fitting}

After classification, events are passed through track fitting programs, which are designed to improved upon the fit obtained in pattern recognition by doing a more complete integration of each track through the non-uniform magnetic field. $\boldsymbol{A}$ track is described by the five parameters $\kappa, \tan \lambda, \phi_{0}, z_{0}$, and $x_{t}$ at the point nearest the beam. These parameters describe a helix, but because of the nonuniformity of the field, the overall track is not helical. However, esch step of the integration covers a small region in which the field is sufficiently uniform that the track segment is a helix to a good approximation.
After the track has been integrated through the drift ehumber regions, for each cell through which it passes the distance of the track from the measured hit position may be calculated. A $x^{2}$ value is formed by the sum of squares of such residuals, with each term divided by the square of the expected error. Correlations between layers are not included, though they can be caused by multiple scattering. For low momentum events, the expected errors for the outer drift chambers are expended to large enough values that those chambers da not contribute very much to the momentum measurement. That is appropriate for two-photon events, since there is a relatively large amount of material before and within the outer drift chambers. The derivatives of $x^{2}$ with respect to the five parameters are approximated analytically, which allows $\chi^{2}$ to be minimized in a linear approximation. That gives a new set of five parameters to be used as a starting point of the next iteration. Also, in each iteration the program has some freedom to choose new hits and to change hits from one side of the sense wire to the other, in case such a solution is found which gives a better fit than the one determined by the pattern recognition programs. However, time does not allow all possible combinations of hits to be tried.

When the track fitting results are complete, they are used as a basis for repeating the procedure of associating tracks :. th counters. That is done because the fit results are more accurate than the pattern recognition results alone. Finally, separate summary tapes are made for the tagged and untagged analyses which include only events with exactly two tracks. The resulting data tapes then are ready for physics analysis. 


\section{The QED Channels}

4.1 Complete Calculations of the Cross sections

There are two common processes in two-photon physics which invalve only electromagnetic interactions. They are

$$
\begin{aligned}
e^{+} e^{-} & \rightarrow e^{+} e^{-} e^{+} e^{-} \\
\text {and } e^{+} e^{-} & \rightarrow e^{+} e^{-} \mu^{+} \mu^{-} .
\end{aligned}
$$

Figure 4.1 shows examples of the leading order $\left(\alpha^{4}\right)$ diagrams which contribute to these processes. When all possible permutations are made, there are a total of thirty-six amplitudes for the four-electron final state and twelve for the $e^{+} e^{-} \mu^{+} \mu^{-}$ final state, all of which must be summed together in each case before taking the square to calculate the cross section. It is prohibitively difficult to square the amplitude and reduce the result analytically to a manageable expression, so in practical calculations the amplitudes are summed numerically before squaring. That makes the algebra more straightforward, but care must be taken to avoid numerical instabilitics.

The full leading-order calculation for both processes has been done with Monte Carlo integration by Berends, Daverveldt, and Kleiss. ${ }^{32}$ The method which they use is described in Appendix B. Their calculations demonstrate that, for the experimental situation where only two of the leptons pass through the central detector, the first two diagrams of Fig. 4.1 (commonly called the multiperipheral diagrams) completely dominate the cross section. In Appendix B, another calculation is made which includes the complete multiperipheral amplitudes, except for interference of the beam electrons with those produced by the two photons, plus four $t$-channel radiative Bhabha scattering diagrams. That calculation is done to a level of statistical significance comparable to that of our data and shows not only that the multiperipheral diagrams alone can fully describe the cross section, but also that for untagged events the equivalent photon approximation can calculate them with sufficient accuracy.
1)

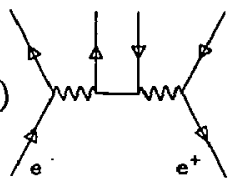

3)

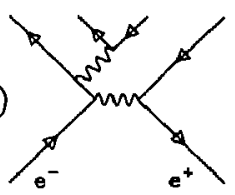

5)

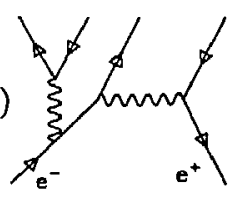

2)

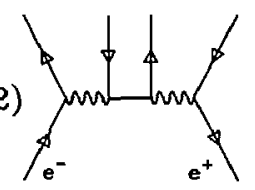

4)

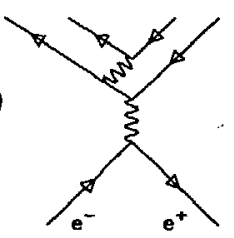

6)

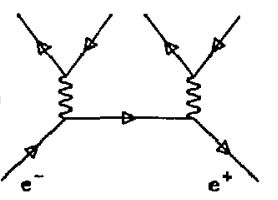

Figure 4.1. The types of diagrams contributing to $e^{+} e^{-} \rightarrow e^{+} e^{-l^{+} l^{-}}$. There are a total of 36 diagrams if $l=e$ and 12 if $l=\mu$.

Thus it is clear that an untagged experiment is sensitive to only two of the thirty-six diagrams for the process $e^{+} e^{-} \rightarrow e^{+} e^{-} e^{+} e^{-}$. That remains largely true even for an experiment with small-angle $(\approx 25 \mathrm{mrad})$ tagging. These two important diagrams are the type which are important to two-photon physics because they represent the interaction of two photons after each has been radiated by opposing electron beams. Therefore we expect the electron-pairs which are observed in the central detector to have kinematic properties which are similar to those of hadron pairs observed in the same manner. That makes the QED measurement especially powerful for nirmalization and calibration of the 
experiment.

\subsection{The DELCO Experimental ACCeptance for $e^{+} e^{-} \rightarrow e^{+} e^{-} e^{+} e^{-}$}

In the untagged analysis, only two of the electrons are observed in the central detector. For such a configuration, the geometric acceptance is determined by the limits of the barrel shower counters, which are necessary for the trigger, and the Cerenkov counters, which are necessary for identifying the electrons. Actually, any track from the interaction point which hits a barrel shower counter will have passed through a Cerenkov cell, so the shower counters always give a more severe limitation. Their coverage of the polar angle is almost the same as that of the Cerenkov system, but they have many azimuthal gaps. The inner tracking chambers, which also are essential to the measurement, have two azimuthal gaps as well, eince layers 7 through 16 separate into two halves along the $y-z$ plane. However, all of the azimutlial gaps have no effect except to cause an inefficiency which is uniform with respect to all of the relevart kinematic variables.

The coverage of the Cerenkov counters is complete within the range $-0.6 \leq$ $\cos \theta \leq 0.6$, which is used in the analysis to define sharply the fiducial volume. The barrel shower counters do not extend quite as far and, in fact, will not intercept all tracks in the interval $0.55<\cos \theta<0.60$. Figure 4.2 shows the loss in efficiency incurred by requiring each particle to be tracked into a shower counter. Whether counter is associated with a track is determined by using the fitted track parameters to provide a starting point for an integration through the magnetic field out to the shower counter module. The exact edge of the counter with respect to $\cos \theta$ will vary from track to track partly because of the spread of several centimeters in $z$ of the beam-beam interaction point, but mostly because the counters are planar-the edge is not at a constant radius from the beam.

Only one electron is necessary for the KS trigger, which requires a Cerenkov cell to fire in coincidence with a shower counter in the same sextant of the detector. Also, it is only necessary to identify one electron before assuming the other particle

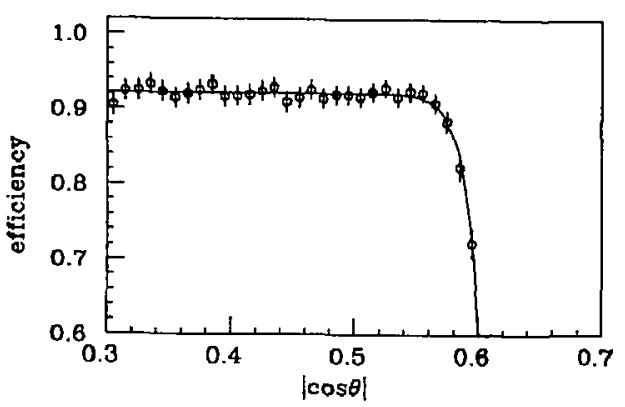

Figure 4.2. The angular acceptance of the barrel shower counters integrated over $\phi$. The points shows the efficiency for a track produced near the interaction point at a polar angle $\theta$ to strike a shower counter.

also to be an electron, so it would be possible to do the experiment within a fiducial volume which requires only one track to fall within the shower counter and Cerenkov acceptance. However, the trigger is easier to understand if both electrons strik: a shower counter module, and the rejection of non-electrons is better if both particles pass through a Cerenkov cell. Since the number of events is so large that the measurement is dominated by systematic rather than statistical uncertainties, we choose to be conservative and require that both tracks fall within the range $-0.6 \leq \cos \theta \leq 0.6$ and that both hit a shower counter.

\subsection{Separation of Electrons from More Massive Particles}

Electron pairs are selected from the two-track data get by using only the Cerenkov counter information. Separation of electrons from more massive particles is much better for datia where isobutane was used as a radiator than for data taken with a nitrogen radiator. That is because the denser gas :adiates more light and thus gives a better efficiency for the low-momentum electrons. It does not matter that nitrogen has a higher Cerenkov threshold for muons, because 
the isobutane muon threshold is above the momentum range relevant to this analysis. Since the isobutane data itself has a high enough statistical weight that the errors are dominated by systematic effects, there is nothing to be gained from including the nitrogen data, which would only introduce larger systematic errors from background and efficiency corrections. Therefore, the analyses of electron pairs and pion pairs are done with only the isobutane radiator.

With the isobutane radiator, the threshold for muons is about $p=1.8 \mathrm{GeV}$. The eross section for any of the particles produced from two photons to have a momentum greater than that threshold within a Čerenkov cell is not large enough to be significant to any of our measurcments. Therefore, only electrons will fire the Cerenkov counters, which are used only for separating electrons from all of the heavier particles.

As we will see, the separation of electrons from muons, pions, kaons, and protons is especially good for the events of interest, which consist of a small number of tracks spaced well apart in $\phi$. However, the small statistical uncertainty in the experiment requires that the efficiency and rejection be understood at the level of about one percent. To do so, the cuts must be chosen to give optimal efficiency and rejection, and an accurate method of determining the efficiency from the data itself must be found.

The firat step of the analysis is to plot the Cerenkov pulse heights and time residuals for both electrons and muons. $\dagger$ Since two-electron events have no ambiguities resulting from multiple particles traversing the same or nearby cells, then it makes sense to include the pulse heights of all cells which the track passes through or nearby. That is especially helpfol near the $z=0$ midplane of the detector. There the efficiency is relatively poor for a combination of reasons. The path length through the radiator is shortest there, the light falls on the mirror edges and is divided between at least two cells, and there is a small dead

$\uparrow$ To aimplify the discuwion, the heavier particles as a group are referred to as muona whenever the distinction between muon, pions, kaona, and protons is not relevant. region between the mirrors. It also is helpful between cells adjacent in $\phi$, where the light tends to be shared between two cells, especially if the track is highly curved. Another important point is that near the edges one cannot be sure from the tracking which cell the particle actually went through. That problem is most severe at the $z=0$ midplane because the dip-angle measurement is not as good as that of the aximuthal angle. A negative result of adding all associated cells together is that the level of random noise is increased, but the gain in efficiency outweighs the loss in rejection caused by noise.

The events which are used to analyze the identification cuts consist of either two electrons or two muons. The QED process $e^{+} e^{-} \rightarrow e^{+} e^{-} \mu^{+} \mu^{-}$can produce a muon and an electron within the central detestor, but the cross section is relatively minute $\dagger$ and is further suppressed by the requirement of balanced transverse momentum $\left(k_{\perp} / W<0.2\right)$. Another possible background is from $e^{+} e^{-} \rightarrow e^{+} e^{-} \tau^{+} \tau^{-}$, but it is heavily suppressed by the kinematic cuts. we assume that in ail cases the two particles are of the same type. That allows a strategy to be used which makes the histograms easier to understand. One of the two particles, chosen at random, is classified as an electron or muon (or neither if the result is ambiguous) by the use of cuts which are designed to give high rejection of background at the expense of good efficiency. Subsequent results verify that cuts can easily be found which result in essentially $100 \%$ rejection of background. If the particie is in one of the two categories, then the other is assumed to be of the same type, and it is entered into one of two plots, depending on whether it is an electron or a muon.

The Cerenkov time residual is used to reject background noise. Fast charged particles coming directly to the phototube from the interaction point produce times centered about zero, whereas the noise is distributed uniformly in time. Figure 4.3 shows a histogram of the best time of all cells associated wit 1 an electron. The

$\dagger$ See Ref. 32 for come example calculations. Fer an acceptance cut of $25^{\circ}<\theta<155^{\circ}$, for example, they calculate $\sigma_{\mu \mathrm{me}} / \sigma_{\mu \mu}=5 \cdot 10^{-4}$.

$\$$ The background from szu-pain is eatimated in detail in Section 4.6. 


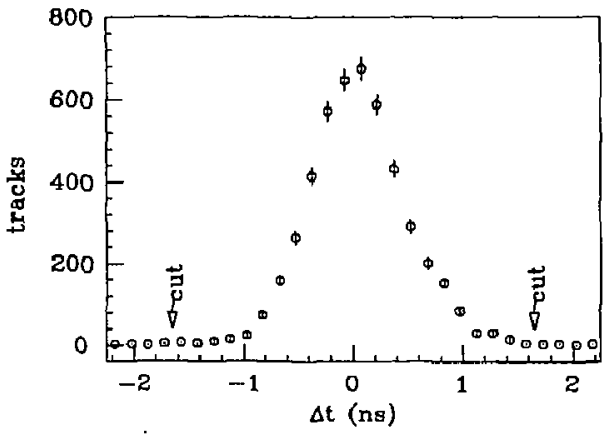

Figure 4.3. The distribution of Čerenkov time tesiduals from electrons.

cuts for the remainder of the analysis are placed at $\pm 1.65 \mathrm{~ns}$ and reject less than $1.4 \%$ of actual electrons.

When the Cerenkov counter is used to select particles with velocities above threshold, it is important for uniformity of the cut to consicier the path length of the track through the radiator and the efficjeлcy of the cell in question. Oл the other hand, if it is used to reject tracks with associated light, then the raw pulse height should be used because the noise level does not depend on the path length. Therefore, when selecting electrons one considers the path-length-corrected pulse height. Figure 4.4 shows the lower end of the distribution for particles which have been assumed to be electrons. About $1 \%$ of the particles give essestially zero pulse height. A small number of these may actually be muons, but we will see that the number of zeroes is consistent with the inefficiency near the detector $z=0$ midplane. That fraction would be rejected by any useful cut, so the best possible efficiency is obtained with a cut requiring the pulse height to be greater than about 1.5 photoelectrons.

When a single track in each event is identified, there is a contamination

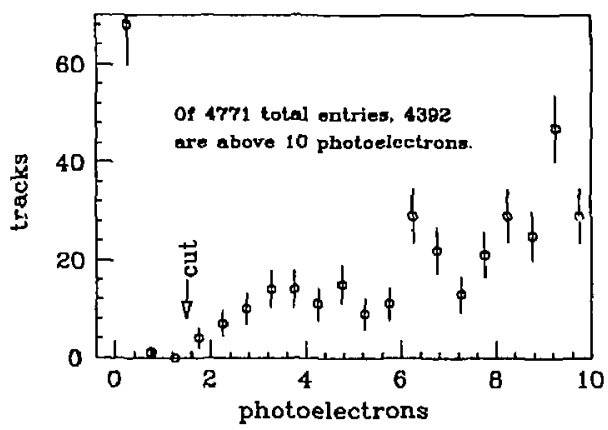

Figure 4.4. The distribution of Cerenkov pulse height for electrons. The pulse heights have been corrected to cancel differences resulting from differing track lengths and different cells.

of muons at the level of one half to one percent due to random noise which forms a narrow peak around a pulse height of one photoelectron. However, when muons with out-of-time raw Cerenkov signals at the level of one photoelectron are used to make a histogram of the path-length-corrected pulse height, the result is that the one-photoelectron noise peak is pushed up to the level of two to three photoelectrons corrected. A cut placed high enough to give complete rejection of muons will seriously compromise the electron efficiency. Furthermore, the analysis requires that both tracks in each event pasa the identification cuts. That increases the rejection at the expense of lowering the efficiency. Therefore, the final decision is to require all electrons to have a corrected Cerenkov pulse height, summed over all associated counters with time residuals between $-1.65 \mathrm{~ns}$ and $1.65 \mathrm{~ns}$, which is greater than 1.5 photoelectrons. Then the contamination of muon pairs is less than $0.01^{2}$, which is completely negligible. 


\subsection{ELBCTRON IDENTIFICATION EFFICIENCY}

The efficiency of the cuts chosen for selecting electrons can be measured in a unbiased way directly from data. The method relies on the fact that a cut on the Cerenkov pulse height placed well above three photoelectrons corrected, which is beyond the limit of the background noise, will reject $100 \%$ of muons. The analysis proceeds by choosing a random track from each event and rejecting the event if that track has an in-time $(|\Delta t|<1.5 \mathrm{~ns})$ pulse height of less than six photoelectrons corrected. The remaining events have an insignificant contamination of muon pairs, so the opposite track in each event can be used to study the efficiency of the analysis cuts. The six-photoelectron cut rejerts only $3 \%$ of electrons overall and introduces no significant bias for the opposite track.

In the following efficiency plots, the points represent for each bin the ratio of the number of electrons which are identified correctly, by the cuts detailed in the previous section, to the total number of electrons. The error bar for a single bin is the statistical uncertainty of the data in that bin only and represents $95 \%$ confidence level limits on the ratio. Figure 4.5 shows the $\cos \theta$ dependence of the electron identification efficiency integrated over all momenta within the acceptance. For the isobutane radiator it is about $99.5 \%$ in all regions except near the $z=0$ midplane. The same plot also is shown for the data sample with the nitrogen radiator, so one can see the serious loss of efficiency caused by the less dense gas. There also is some momentum dependence, with most of the inefficiency resulting from low momentum particles. That is a result of the large curvature of the tracks lowering the light gathering efficiency of the optical system, which was designed to focus light coming straight from the interaction point.

Rather an use a smooth two-dimensional parameterization of the efficiency, the angular range is divided into $\cos \theta$ bins and the momentum dependence is parameterized for the central bins by an exponential:

$$
\varepsilon(p)=A \cdot\left[1-0.6 \exp \left(-\frac{p-p_{0}}{\tau}\right)\right] .
$$

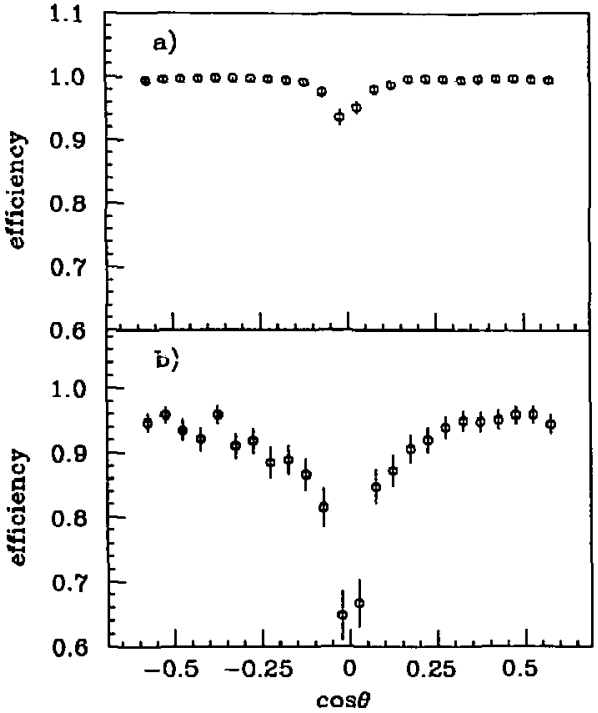

Figure 4.5. The efficiency for electron identification as a function of $\cos \theta$ and in tegrated over all momenta. (a) with the isobutane radiator. (b) with the nitrogen radiator.

The fitted parameter values are listed in Table 4.1. Note that these parameterizations represent the efficiency for a single track, so the efficiency for both tracks to be identified, as required in the analysis, is lower.

\subsection{Trigger EFficiency for Electron PaIRS}

To determine the efficiency of the KS trigger, one somehow must measure the latch efficiencies of the Cerenkov and shower counters. Judging from the measured electron identification efficiency of the previous section, we expect the Cerenkov 
Table 4.1. A parameterization of the electron identification efficiency. The symbols $A, p_{0}$, and $\tau$ refer to variables in Eqn. 4.2.

\begin{tabular}{|c|c|c|c|c|}
\hline$|\cos \theta|_{\min }$ & $|\cos \theta|_{\max }$ & $A$ & $p_{0}$ & $r$ \\
\hline 0.0 & 0.05 & 0.975 & -0.092 & 0.15 \\
0.05 & 0.10 & 0.890 & -0.280 & 0.15 \\
0.10 & 0.60 & 0.995 & - & - \\
\hline
\end{tabular}

lateh efficiency to be close to unity except at the $z=0$ midplane, since the lateh threshold is lower than the cuts used for electron identification. However, the shower counter latch, which requires two of three leyere to letch, will not fin unleas the electron penetrates through at least fivo radiation lengthe of lead. The probubility for that is significantly leas than unity in the lower end of the momentum range of interest.

The Cerenkov latch efficiency is easily menured from date without any bias from the trigger itself. Only events with $a 2 S$ trigger are used. From these, electron pairs are chooen by identifying one particle, chowen at random from the pair, with tight cuts on the Cerenkov pulse height, just an was done for the messurement of the identification efficiency. In this case, though, the identified electron is required to be well away from the $z=0$ midplane in order to avoid any bias from the identification cut. There is no prowibility of bia in the region away ftom the midplane, because for $|\cos \theta|>0.10$ the effieiency is ensentially $100 \%$.

The particle which is not ident:fied lo used to accumulate histograms of the latch efficiency. Figure 4.6 shows the $\cos \theta$ dependence with the full momentum range included. It verifies that the efficiency is essentially $100 \%$ when away from the region near $\cos \theta=0$. Fitting the momentum dependence of the central $\cos \theta$ bins to the parameterization given by Eqn. 4.2 yields the set of parameters presented in Table 4.2.

Whilo we have seen that the Cerenkov latch has only a small and almoet

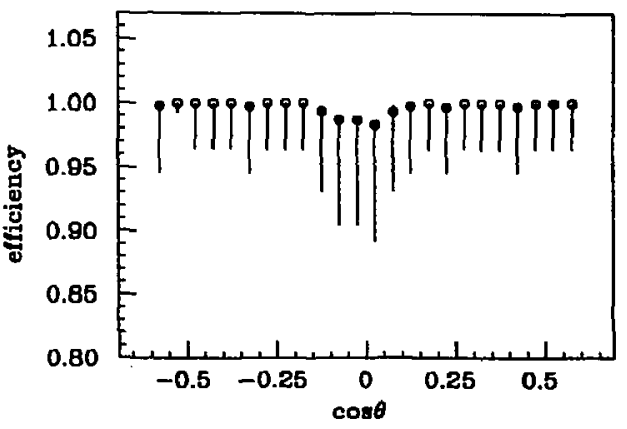

Figure 4.6. The Cerentov latch efficiency is \& function of $\cos \ell$. The full momentum range is included.

Table 4.2. A parameterization of the Cerenkov lateh efficiency. The aymbols A, Po, and $r$ refer to variables in Eqn. 1.2

\begin{tabular}{|l|c|c|c|c|}
\hline$|\cos \theta|_{\min }$ & $|\cos \theta|_{\max }$ & $A$ & $p_{0}$ & $T$ \\
\hline 0.0 & 0.025 & 1 & 0.131 & 0.072 \\
0.025 & 0.050 & 1 & 0.030 & 0.072 \\
0.050 & 0.600 & 1 & - & - \\
\hline
\end{tabular}

negligible inefficiency, that is not the case for the shower counter latch. It is especially important to understand the shower counter efficiency because every trigger requires at least one shower counter to lateh. It can be meanured from the data becsuse the trigger is Jedundant; only one of the two electron track mut fire a shower counter. Therefore, the effieiency can be seen directly by colectins electron pairs in which both particles hit a shower counter and simply accumulating efficiency histograms using the latch information of both hit counters. The renult is, however, biased by the trigger, because it is not possible to include in the 
histograms any events in which both electrons have failed to latch a shower counter. That bias must be corrected by Monte Carlo calculations.

For the moment wo concern ourselves only with inefficiency caused by lack of penetration of electromagnetic showers. Geometric ineficiency is more simple to understand and does not have any momentum dependence. Therefore, histograms are accumulated using only events in which both tracks are projected to hit well within ( $\geq 3 \mathrm{~cm}$ ) the boundaries of a shower counter module.

Figure 4.7 and Fig. 4.8 show the trigger-biased latch efficiencies as functions of the mesured electron momenta for the three individual shower counter layers, where only data taken in $198 \mathrm{are}$ ased. $\dagger$ Figure $4.9 \mathrm{a}$ shows the reault for the shower counter latch, which requires two of the three layers to fire. Note that the trigger biag is such that the true latch efficiency is lower than that which is observed. The measured efficiencies are compared with calculations of the same quantities using the EPA Monte Carlo for $e^{+} e^{-} \rightarrow e^{+} e^{-} e^{+} e^{-}$and a full simulation of detector effects. The shower counter response has been simulated by the EGS Monte Carlo? 20 y using EGS one can do a reasonable job of simulating the propagation and development of the electromagnetic shower through the aluminum, lead, and scintillator. However, when dealing with a sandwich of thin layers of scintillator between sheets of lead it still is difficult to predict with high accuracy how much of the deposited energy actually is collected by the scintillator. Furthermore, there are a number of additional effects which are not handled in much detail. For example, fluctuatio.ss in light transmission and phototube response are not simulated at all, and the response of the electronics is treated in a simplistic manner. Therefore, one cannot expect perfect agreement of Monte Carlo with data, and it is the measured efficiency which is used in the ensuing anslysis. Nonetheless, the extent of the agreement shown in figures 4.7

† During the summer of 198s, the gain of the phototubes was lowered to reduce saturation from large pule heights. The change was compenated by installing amplifiers in front of the lateh olectronich, but the latch efficiency changed alightly nonetheless.

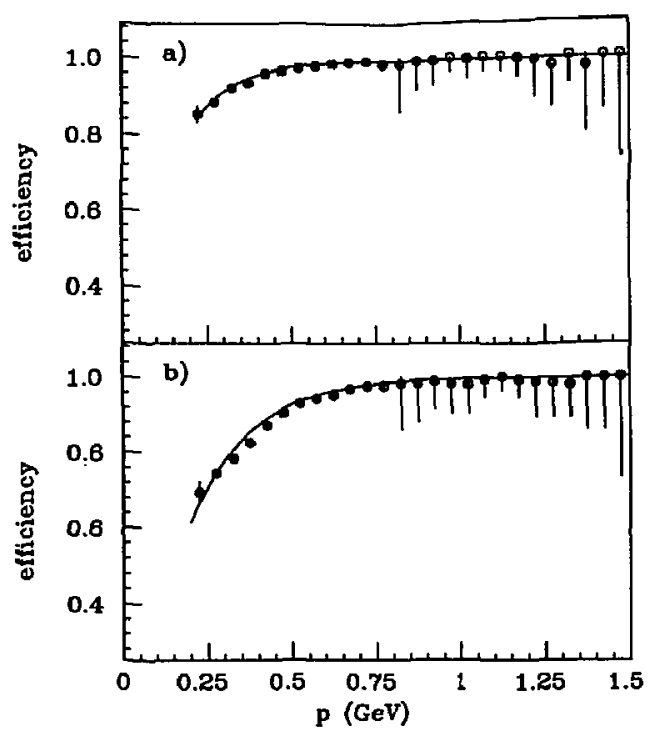

Figure 4.7. The shower counter layers-1,2 biased latch efficiency as a function of the measured momentum for electrons hitting well within the counter boundaries. Figure $(a)$ is for layer-1 and $(b)$ is for layer-2. .The smooth curves represent the EGS Monte Carlo calculations.

through 4.9 is remaskable and indicates that the reason for the inefficiency is well understood and the mensurements are correct.

One expects some dependence of the efficiency on the angle of incidence $\lambda$ of an electron on the counter, because the amount of material that the shower must traverse increases with $\lambda$. It is not obvious how much of an effect to expect, however, because once the electron enters the lead, the shower does not propagate only in the original direction of the electron. Further complications are that 


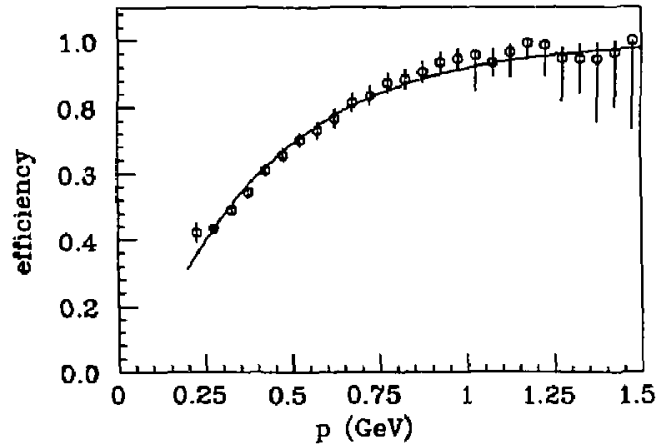

Figure 4.8. The shower counter layer-3 biased latch efficiency as a function of the measured momentum for electrons hitting well within the counter boundaries. The smooth curve represents the EGS Monte Carlo calculation.

the effert may be compensated to some extent by light attenuation and that $\lambda$ depends on the momentum through the curvature produced by the magnetic field. Figure 4.10 shows the measured efficiency as a function of the angle of incidence. There is only a small deviation from fatness at the largest angles, and in that region the number of events is relatively small. Variations in efficiency with the angle of incidence come from three sources. It varies directly with $\cos \theta$, and it also varies with $\phi$ because of the hexagonal shape of the shower counter. Then there is some additional variation due to the curvature of the tracks. The latter effect ss included in the momentum dependence and must not be double counted. It is not important to include the $\phi$ dependence of the angle of incidence in detail, but only as an effect uniform with respect to the relevant kinematic variables. That leaves only the dependence on $\cos \theta$ to be concerned with, and in fact no such dependence can be seen in the data or Monte Carlo with the available statistical weight. There is, however, an additional inefficiency of a few percent in the $\cos \theta$

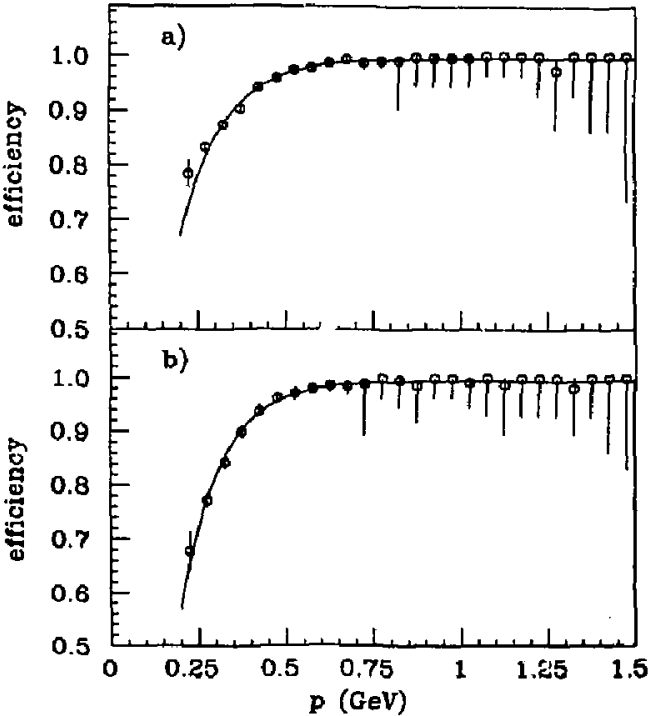

Figure 4.9. The shower counter latch efficiency as a function of the measured rinomentum for electrons hitting well within the counter boundaries. (a) The measured trigger-liased efficiency compared with a smooth curve representing the EGS Monte Carlo calculation. (b) The unbiased efficiency as calculated by EGS and fitted to an analytic function.

bins nearest the center of the detector. That is understood to be primarily a geometric ineficiency resulting from the small gap at $z=0$ in layers two and three between the scintillators covering the $+z$ half of the detector and those covering the $-z$ half. It is accounted for by treating separately the regions $|\cos \theta| \leq 0.05$ and $|\cos \theta|>0.05$.

The Munte Carlo must be used to unfold the true lateh efficiency from what has been observed. The goal is to arrive at a parameterization which tescribes 


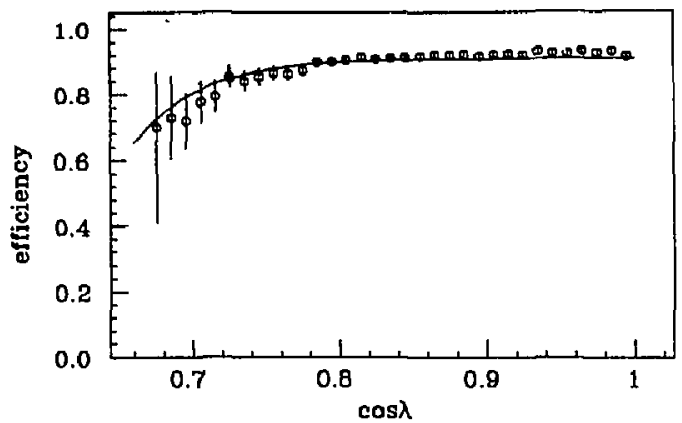

Figure 4.10. The measured tor: ${ }^{2}$ er-bisis ${ }^{3}$ ahower-counter latch efficiency as a function of the angle of iuence $c f t$, electron on the counter. The smooth curve represents the $1, a^{-}, \ldots$ Carlo calculation.

the momentum dependence of the unbiased latch efficiency with an accuracy as good as the statistical precision of the data. That is done in two steps. First, the EGS Mante Carlo is used to calculate the unbiased latch efficiency as shown in Fig. 4.9b, and fitting it to the form of Eqn. 4.2 yields the results $A=0.995$, $p_{0}=0.161$, and $r=0.116$.

To achieve the required precision, the parameterization must be adjusted to give the best possible agreement with the histogram of the biased latch efficiency measured from data. As already mentioned, the efficiency varies slightly in the data over time, so what is done is to adjust the parameterization until agreement is reached with a histogram accumulated using all of the data. Thus the result represents an average efficiency.

The trigger bias in the latch efficiency depends on the momenta of both particles, so it is not trivial to calculate the biased efficiency when given the parameterization of the actual efficiency. What is done is to ure the EPA Monte Carlo event generator to produce events with the same momentum and angular distributions as are observed in the data. For each Monte Carlo electron track, the efficiency parameterization is used along with a uniform random number to decide whether the shower counter will latch. Then a histogram of the biased lateh effieiency can be accumulated, just as is done for the data, as a function of the clectron momentum. That is done for each of 100 pairs of values for $p_{0}$ and $\tau$ forming a $10 \times 10$ grid about the values obtained from the EGS shower simulation, and the pair is chosen which gives the best fit. Figure 4.11 shows the result, where the Monte Garlo distributions giving the best fits have been plotted over the data. The final values for the parameterization of the shower counter latch efficiency are given in Table 4.3 .

\subsection{SUMmary of GUTS MADE ON UNTAgged Electron PAIRS}

Many cuts were made on the data, but only a few are very significant in the sense that they reject a sizable fraction of the events. No background of any sort can be seen in the sample of electron pairs from data, and it is difficult to conceive of any source of background beyond the negligible contamination of muon pairs which sneak in due to noise in the Cerenkov counters. For example, let us consider radiative Bhabha acattaring. For the two final-atate electrons to be within the angular acceptance, the boost of the final $e^{+} e^{-}$syatem must have $-0.6 \leq \beta \leq 0.6$. That limit is reached if one of the incoming beam electrons radiates $75 \%$ of its energy, and the resulting minimum invariant mase of the final $c^{+} e^{-}$pair is $14.5 \mathrm{GeV}-$ par enough above the $2.6 \mathrm{GeV}$ cut used in this analysis to give absolutely no background. If both beams radiate photons of equal energy, then the final $e^{+} e^{-}$pair could have a mass below the cut. That process is the same order in $\alpha$ as the four-electron final state, but in order for both electrons to be scattered into the DELCO acceptance, the two radiated photons would have to have large and almost equal energies. Although a complete calculation has not been done, the cross section into the DELCO acceptance is believed to be negligible. 


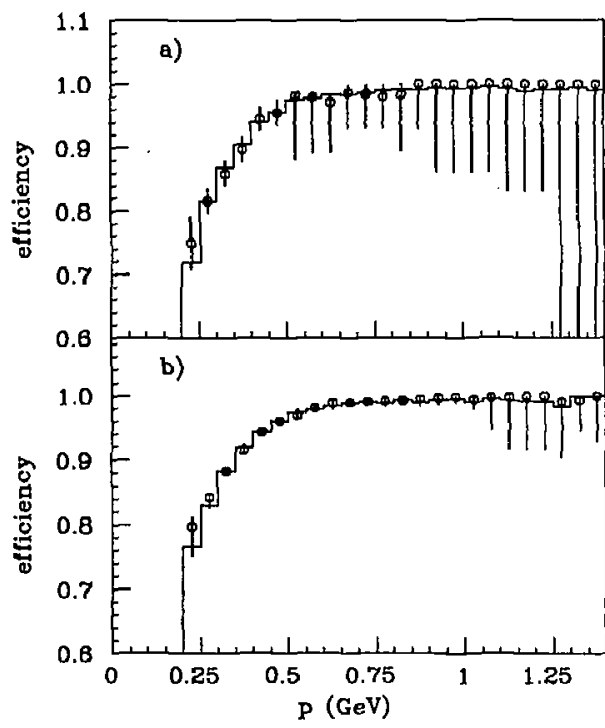

Figure 4.11. The biased shower counter latch efficiency as a function of the electron momentum, including all available data. The solid histograms are from a Monte Carlo calculation including a simulation of the trigger, where the input unbiased latch efficiency is taken to be the parameterization which yields the best fit $(a)$ for $|\cos \theta| \leq 0.05$ and (b) for $|\cos \theta|>0.05$

A nother possible source of background is tau-pair production. The MonteCarlo generator used to estimate it includes QED radiative corrections, ${ }^{33}$ weak interactions, and all known decay channels for the tau ${ }^{34}$ of 4508 tau-pairs generated and simulated in the detector, only one produces an electron pair which passes all of the analysis cuts. When normalized to the integrated luminosity of the data, this gives an estimate of only four background events from tau-pair
Table 4.3. A parameterization of the shower counter latch efficiency. The symbols $A, p_{0}$, and $\tau$ refer to variables in Eqn. 4.2.

\begin{tabular}{|c|c|c|c|c|}
\hline$|\cos \theta|_{\min }$ & $|\cos \theta|_{\max }$ & $A$ & $p_{0}$ & $\tau$ \\
\hline 0.0 & 0.05 & 0.995 & 0.160 & 0.116 \\
0.05 & 0.60 & 0.995 & 0.154 & 0.110 \\
\hline
\end{tabular}

production, which clearly is negligible.

Because there is no background, the cuts made after separation of electrons from muons have only two purposes. Most serve to define the kinematic region to be observed and the usable fiducial region of the detector. Then there are some which only discard long, low tails in the distributions of some measured variables in order to avoid events with large, non-gaussian measurement errors.

The last category is comprised of several cuts on the tracking of charged particles. Each track is required to have associated with its fit at least 12 total hits in the inner cylindrical tracking chambers, and track fitting must be completed without any catastrophic errors. Each track must project back to within $0.5 \mathrm{~cm}$ of the bearnline, and the average of the $z$-positions of the points on the tracks closest to the beamline must be within $4.0 \mathrm{~cm}$ of the interaction point. The cuts on the total transverse momentum, $k_{\perp}<0.3 \mathrm{GeV}$ and $k_{\perp}<0.2 W$, also reject poorly measured events.

The fiducial volume is defined by requiring each track to have $|\cos \theta|<0.6$ and to intersect a shower counter module. The cuts on $k_{\perp}$ along with the requirement that there be no more than $4 \mathrm{GeV}$ of energy measured in the luminosity monitors restrict the measurement to events with quasi-real photons. The Pass-1 filter cut which requires at least one track with a rough $p l$ measurement, from the first stage of pattern recognition, greater than about $0.20 \mathrm{GeV}$ is superseded for simplicity by a hard cut requiring at least one track to have a fitted transverse momentum greater than $0.25 \mathrm{GeV}$. Finally, the kinematic range which is accessible without 
unreasonably large trigger effects is $W \geq 0.6 \mathrm{GeV}$. The upper limit on $W$ is roughly determined by the decrease in statistics with higher energies and by the muon Cerenkov threshold and is set at $2.6 \mathrm{GeV}$. Table 4.4 lists all of the cuts along with the number of events rejected by each. The cuts listed in Table 4.4 have been preceded by those of the $P$ ass- 1 filter and the two-photon classification-filter, but in all cases the Pass-1 and classification cuts are superseded by the final analysis cuts.

\subsection{The Monte Carlo stmulation}

After making the cuts on the data as listed in the previous section, it is possible to simulate adequately all asperts of the detector response relevant to the kinematic distributions without making a detailcd simulation of all of the detector apparatus. That has the advantage of requiring far less computer time, which can be significant when so many events are involved, but more important is the fact that no program exists which can properly reproduce, from first principles, the Cerenkov and barrel-shower efficiencies to the accuracy required. Instead, those have been measured from data, and it is a simple matter to put the resulting parameterizations directly into the Monte-Carlo integration.

To proceed, the EPA Monte-Carlo program is used to generate evento of equal weight, using the cross section for production of relativistic electron pairs from pairs of real photons:

$$
\frac{\mathrm{d} \sigma}{\mathrm{d} u \mathrm{~d} W}=\frac{2 \pi \alpha^{2}}{W^{2}} \cdot \frac{1+u^{2}}{1-u^{2}}, \quad u \equiv \cos \theta_{\mathrm{cma}}
$$

The momenta are smeared by gaussian random numbers, using the widths determined in Section 3.1. The trigger is simulated by using the parameterizations of tables 4.2 and 4.3 for the Cerenkov and barrel-shower latch efficiencies. In each case, a uniform random number is generated between 0 and 1 , and the event is rejected if it is greater than the efficiency calculated from the parameterization. Similarly, the electron identification efficiency is simulated according to the
Table 4.4. List of the final cuts for $e^{+} e^{-} \rightarrow e^{+} e^{-} e^{+} e^{-}$. A total of 398,936 events were read from the tape of skimmed two-particle events, and 41,328 pass all of the cuts. Excepting the first and the last two cuts, the fourth and fifth columns give respectively how many events would be killed by the cut before all other cuts and how many would be killed after all others. "BSC acceptance" refera to the requirement that both tracks pass through barrel shower counter modules, as determined by the drift chamber tracking.

\begin{tabular}{|c|c|c|r|r|r|}
\hline Quantity & $\begin{array}{c}\text { Lower } \\
\text { limit }\end{array}$ & $\begin{array}{c}\text { Upper } \\
\text { limit }\end{array}$ & $\begin{array}{c}\text { Failures } \\
\text { when first }\end{array}$ & $\begin{array}{c}\text { Failures } \\
\text { when last }\end{array}$ & $\begin{array}{c}\text { Failures } \\
\text { in order }\end{array}$ \\
\hline Cerenkov ph. & $1.5 \mathrm{pe}$ & $\infty$ & & & 217405 \\
\hline $\bar{z}$ & $-4.0 \mathrm{~cm}$ & $4.0 \mathrm{~cm}$ & 408 & 86 & 408 \\
\hline $\begin{array}{c}\text { impact } \\
\text { parameter }\end{array}$ & 0 & $0.5 \mathrm{~cm}$ & 5823 & 234 & 5773 \\
\hline fit errors & 0 & 0 & 220 & 22 & 150 \\
\hline inner DC hits & 12 & 16 & 3950 & 883 & 3129 \\
\hline MAX $\left(p_{\ell}^{1}, p_{\ell}^{2}\right)$ & $0.25 \mathrm{GeV}$ & $\infty$ & 50120 & 1 & 47304 \\
\hline$k_{\perp}$ & 0 & $0.3 \mathrm{GeV}$ & 7541 & 257 & 6714 \\
\hline$k_{\perp} / W_{e c}$ & 0 & 0.2 & 50184 & 7273 & 29346 \\
\hline cos $\theta$ & -0.6 & 0.6 & 29011 & 11598 & 15398 \\
\hline$W$ & $0.6 \mathrm{GeV}$ & $2.6 \mathrm{GeV}$ & 105743 & 23155 & 23695 \\
\hline$p$ & 0 & $1.7 \mathrm{GeV}$ & 815 & 3 & 3 \\
\hline BSC acceptance & & & & & 8253 \\
\hline$\sum_{i} Q_{i}$ & 0 & 0 & & & 2 \\
\hline
\end{tabular}


parameterization of Table 4.1, and the effect of requiring each track to intercept a shower counter is reproduced by the parameterization

$$
\varepsilon(p)=0.092 \cdot\left[1-0.6 \exp \left(-\frac{(0.65-\cos \theta)-0.043}{0.012}\right)\right],
$$

which is obtained from a fit to Fig. 4.2 .

Finally, kinenatic cuts are made just as they were for the data. In summary, both particles must have $-0.6 \leq \cos \theta \leq 0.6$, and at least one particle must have a transverse momentum greater than $0.3 \mathrm{GeV}$. The vector sum of the transverse momenta must satisfy $k_{\perp}<0.3 \mathrm{GeV}$ and $k_{\perp} / W<0.2$, and the electron-pair invariant mass is restricted to the range $0.6 \leq W \leq 2.6 \mathrm{GeV}$. The histograms resulting from simulated events may be compared directly with those from data. As examples, Fig. 4.12 shows the electron-pait invariant mass distribution, and Fig. 4.13 showg the angular distribution of the clectrons in the laboratory and y c center-of-mass systems. Also, in Fig. 4.13, $\cos \theta_{\text {lab }}$ for each entry is multiplied by the mensured charge to demonstrate the charge aymmetry. There are 41,328 events from the data sample and twice that from Monte Carlo. To compare the distributions, the Monte Carlo result simply is renormalized to the same number of events as in data, because there is no process by which the integrated bearn luminosity can be measured accurately enough to allow an absolute normalization. In fact, it is this QED process which is used to normalize all other measurements in this study.

Figures 4.12 and 4.13 indicate that the data do agree well with the theory. However, it is desirable to present the final results in a way which is independent of the many small details of the acceptance which are peculiar to this expetiment.

\subsection{UNFOLDING DETECTOR EFFECTS}

It is useful to present the measured spectra with corrections made for systematic detector effects. However, we do not wish to go to extremes and

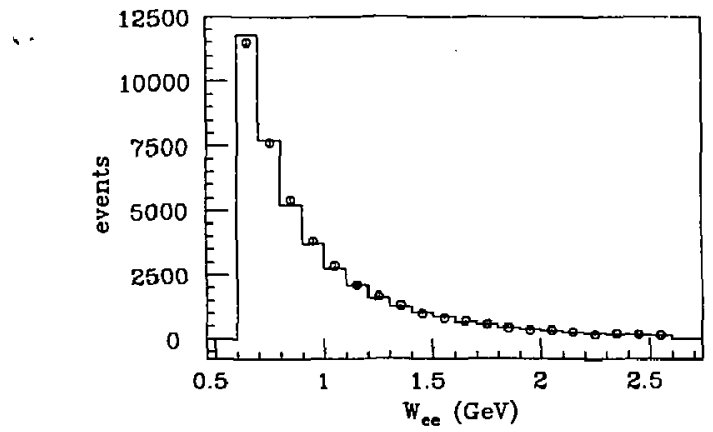

Figure 4.12. The electron-pair invariant mass distribution with al detector effects included. The points are from data, while the line represents the Monte Carlo simulation.

try to extrapolate the data to an acceptance of $4 \pi$ steradians or down to the minimum invariant mass, for what has been measured is only a very small part of the total cross section of $10^{7} \mathrm{nb}$. Instead, it is appropriate to specify only a minimal number of acceptance cuts within which the theoretical distributions are to be calculated. Then by correcting for all detector effects except for those acceptance cuts, the desired distributions are unfolded from the data. Since there is no absolute normalization which can be used, the results simply are normalized to the areas under the distributions. Therefore, the absolute scale of cross section displayed on a plot does not represent a measurement but only the theoretical calculation. Only the shapes of the distributions have been measured to high precision.

For untagged electron-pair production, there are only five independent kinematic variables, which are taken to be the set $Q_{i}^{2}, W, y$, and $\cos \theta_{\mathrm{cms}}$. The $Q_{i}^{2}$ contribute significantly only to the distribution of $k_{\perp}$, but that one is very sensitive to resolution effects which are difficult to understand and dominate over 


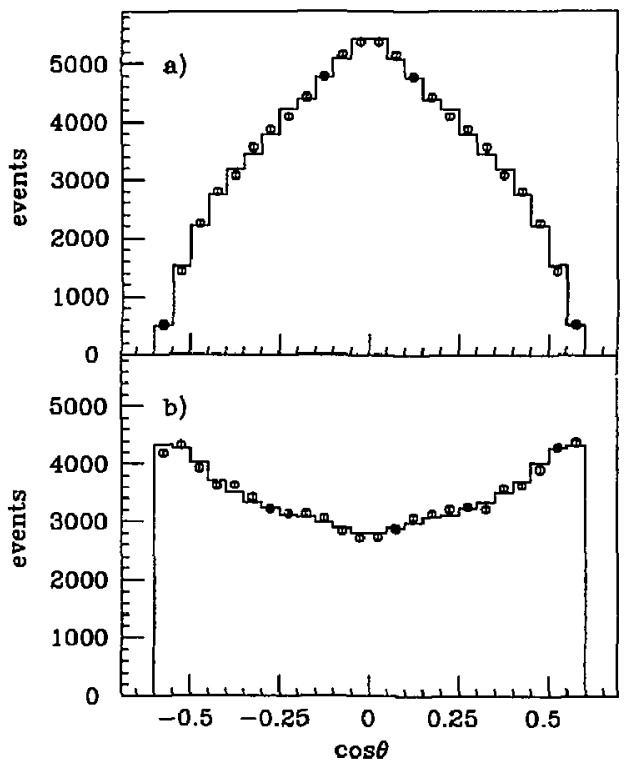

Figure 4.13. The electron-pair angular distributions in (a) the $\gamma \gamma$ centerof-mass system and (b) the laboratory system, with all detector effects included. The points are from data, while the lines represent the Monte Carlo simulation.

the $Q^{2}$ contribution.t Therefore, no attempt is made to unfold the $Q^{2}$ dependence from the $k_{\perp}$ distribution.

It is convenient to use instead of the rapidity, $y$, a related variable defined by

$$
\tilde{\beta}=\frac{\cos \theta_{1} \sin \theta_{2}+\sin \theta_{1} \cos \theta_{2}}{\sin \theta_{1}+\sin \theta_{2}} \text {. }
$$

† See section Section 3.1 for a complete discusuion of this problem.
For the case of minimal $Q^{2}$ and ultra-relativistic clectrons, $\tilde{\beta}$ is the velocity of the $\gamma \gamma$ system, and $y=\tanh ^{-1} \dot{\beta}$. It has the advartage of being defined an;; in terms of angles, which DELCO can measure much better than momenta.

The $W$ and $\ddot{\beta}$ distributions are unfolded into the acceptance defined by

$$
\begin{gathered}
0.6 \leq W \leq 2.6 \mathrm{GeV} \\
-0.6 \leq \cos \theta_{\text {lab }} \leq 0.6 \\
k_{\perp}<0.3 \mathrm{GeV} \\
k_{\perp} / W<0.2 .
\end{gathered}
$$

The EPA Monte Carlo and sirnulation discussed in the preceding section are used to generate an unfolding matrix for each kinematic variable. Each column of such a matrix describes the effects of the experimental resolution and acceptance on a single cubic basis spline. The unfolding program uses the matrix and the corresponding experimental histogram as input and produces the unfolded distribution from those. $f$ The results are compared with QED predictions obtained by running the EPA Monte Carlo (identical results are obtained from the Vermaseren Monte Carlo ${ }^{22}$ ) with on!y the cuts defined by Eqn. 4.6. The data points which result from the unfolding procedure do not directly correspond to the bins used to accumulate the histograms of Monte Carlo or datz and are not even equally spaced. So to compare with data, the Monte Carlo histogram first is interpolated by cubic splines and then integrated over each of the regions defined by the spacing of the points of the unfolded data. That produces Monte Carlo points which directly correspond to those in the data, and from these the $\chi^{2}$ is calculated to give a measure of the agreement. The difference of the data and Monte Carlo is plotted to give a visual display of the agreement, and the data points also are shown with an overlay of the smoothed Monte Carlo distribution.

I See Appendix $\mathrm{C}$ for a detailed description of the unfolding procedure. 
Figure 4.14 shows the final results for the electron-pair invariant-mass distribution. Note that for all such plots, the horizontal bar associated with a point represents only the region spanned by the point and is not an error bar. The vertical bars give estimates of statistical errors, which vary from about $1 \%$ at $W=0.6 \mathrm{GeV}$ to $10 \%$ at $W=2.6 \mathrm{GeV}$. For this case, $\chi^{2}$ is 19.4 for 14 degrees of freedom (one degree of freedom is lost because of the normalization), which is at the $85 \%$ level of the cumulative $\chi^{2}$ distribution. If the QED calculation is assumed to give the correct description of the data and the errors truly have gaussian distributions with widths given by the error bars, then there is a $15 \%$ probability for the $x^{2}$ value to be larger than what has been measured.

For the shape of the invariant mass distribution, the dominant systematic error comes from the correction necessitated by the trigger inefficiency. That error is limited by the statistical accuracy to which the trigger efficiency can be measured by using all of the data and is about $1 \%$ for the first invariant-mass bin. That is, not surprisingly, the same as the statistical ersor on the first bin of Fig. 4.14. In fact, it is a good approximation to obtain the total error for each invariant-mass bin by adding in quadrature with each statistical error an equal systematic error. The agreement between Monte Carlo and data is fairly good even with only statistical errors included, so it is clear that after adding systematic errors there is no evidence for any discrepancy between the data and QED.

Figure 4.15 shows the final results for $\bar{\beta}$, the velocity of the electron-pair Bystem along the beam axis. This quantity is, to an excellent approximation uncorrelated with the invariant mass, so it makes sense to quote a value of $x^{2}$ in addition to and independent of that already given for the invariant mass. For 14 degrees of freedom, the $\chi^{2}$ is 10.4 , which is at the $27 \%$ level of the cumulative distribution.

For the $\cos \theta$ distribution in the laboratory system, there are some systematic errors in the shape near the center and the two ends due to corrections for the

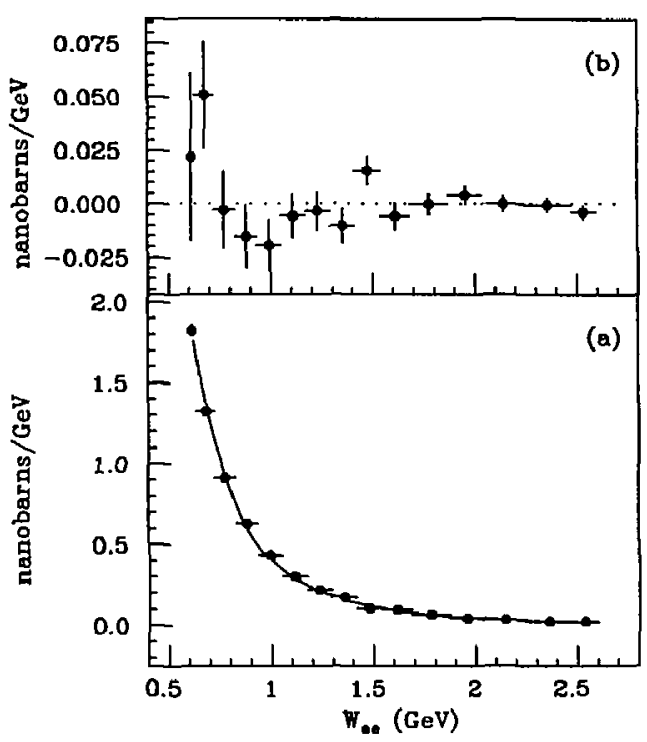

Figure 4.14. The electron-pajr invariant-mass distribution with most detector effects unfolded. (a) The difference of the data and the QED prediction calculated by the EPA Monte Carlo. (b) The data points compared with a smooth curve representing the QED prediction.

detections efficiency, which has significant variations in those regions. The errors in the corrections are only about $2 \%$ in the center $(|\cos \theta| \leq 0.05)$ and $1 \%$ at the ends $(|\cos \theta|>0.55)$. Such errors have no observable effect on the shapes of the angular distribution in the center-of-mass sysiem and the distribution of $\tilde{\beta}$, since the limited region of error in $\cos \theta$ gets mapped over a large region of the other variables. Therefore, we expect that the $\bar{\beta}$ distribution should agree well with QED after considering only statistical errors, and that is indeed the case. Again 


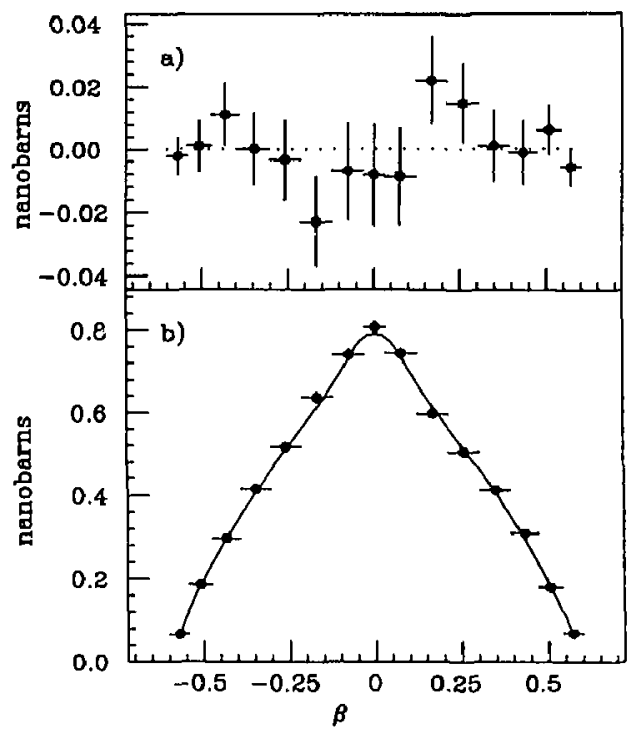

Figure 4.15. The velocity, $\tilde{\beta}$, of the $\gamma \gamma$ electron pair with most detector effects unfolded. (a) The difference of the data and the QED prediction calculated by the EPA Monte Carlo. (b) The data points compared with a smooth curve representing the QED prediction.

there is no evidence for any discrepancy between the data and QED.

The last result to present is for the center-of-mass angular distribution. For this case it is desirable to see the results free from the effect of the acceptance cut made on the laboratory angle. Therefore the results are unfolded into an acceptance the same as defined in Eqn. 4.6, except that the cut on the laboratory angle is replaced by a cut on the center-of-mass angle: $-0.6 \leq \cos \theta_{\mathrm{cms}} \leq 0.6$. Figure 4.16 shows the result, which is compared with the theoretical shape given

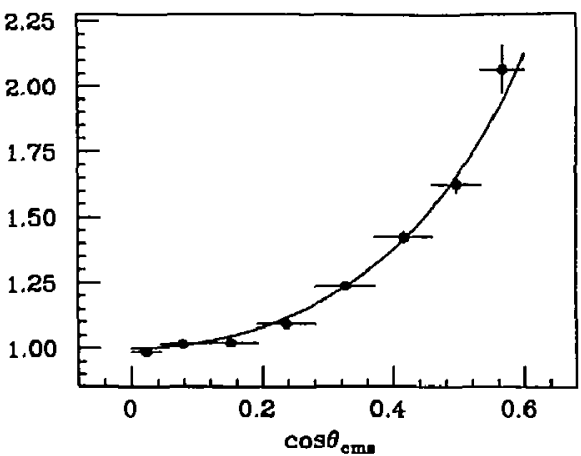

Figure 4.16. The center-of-mass electron-pair angular distribution with most detector effects unfolded from the data. The smooth curve is given by $\left(1+\cos ^{2} \theta\right) /\left(1-\cos ^{2} \theta\right)$.

by Eqn. 4.3. It is clear that even with a se:ere acceptance cut made in the Jaboratory frame, the high statistics available allows us to see quite clearly the center-of-mass angular distribution, which is in excellent agreement with QED.

\subsection{Measurements of TAgged $e^{+} e^{-} \rightarrow e^{+} e^{--} e^{+} e^{-}$Events}

The sample of untagged events is dominated by collisions of quasi-real phatans with $Q^{2}$ not much larger than the square of the electron mass. In order to study collisions of more virtual photons without any contribution from pairs of quasi-real photons, it is necessary to be able to detect at least one of the scattered beam electrons. Lecause of the factor of $1 / q_{1}^{2} q_{2}^{2}$ in the cross section given by Eqn. 2.12, to get a reasonable level of statistical precision, the analysis is restricted to events with a single luminosity counter tag. 
4.9.1 Analysis of the Luminosity-Counter Energy

In DELCO, the only devices able to detect particles at very small angles with respect to the beam are the counters of the Iuminosity monitor. The inner and outer counter aurfaces parallel to the beamline form hexagons about the beam pipe, and the counter surface into which the scattered electrons are incident is perpendicular to the beamline and subtends a range of polar angles, with respect to the interaction point, from $27 \mathrm{mrad}$ to $94 \mathrm{mrad}$. $\dagger$ That corresponds to a range of $Q^{2}$ from $0.13 \mathrm{GeV}^{2}$ to $1.9 \mathrm{GeV}^{2}$. However, each counter is a lead-scintillator sandwich segmented into six azimuthal segments with no radial segmentation, and there is no tracking information in front of the counters. Therefore, it is not possible to measure from the luminosity-counter tag what is the $Q^{2}$ of the radiated virtual photon, except to say that it is between the above limits.

There is some noise background in the luminosity counters, primarily from synchrotron radiation, but even without any charged-particle tracking, the signal is well separated from the noise. The algorithm uscd to analyse the luminosity counter energy deposit is simple. For each counter, one at each end of the detector a scarch is made over the six sextants to find the one with the largest energy deposit. The energy then is summed over that sextant plus the two adjacent sextants to give the quantity used in the analysis. Figure 4.17 shows a histogram of the luminosity counter energy deposit for four-electron events. The counters have been calibrated such that the position of the peak remains constant with time, but the absolute energy scale is somewhat arbitrary. In fact, the Vermaseren QED Monte Carlo ${ }^{22}$ predicts that the median energy of the scaitered electrons, for tagged events within the DELCO analysis acceptance, is $13.7 \mathrm{GeV}$, with $95 \%$ of the energy deposits within $0.6 \mathrm{GeV}$ of that value. A Monte Carlo calculation for Bhabha scattering, including radiative effects, ${ }^{33}$ predicts that the median energy deposit for Bhabha electrons is $14.45 \mathrm{GeV}$, with $95 \%$ of the energy deposits above

† These limits refer to the inscribed and circumacribed radii of the scintillation counter covering the entivo shower counter face into which the telestrons are incident.

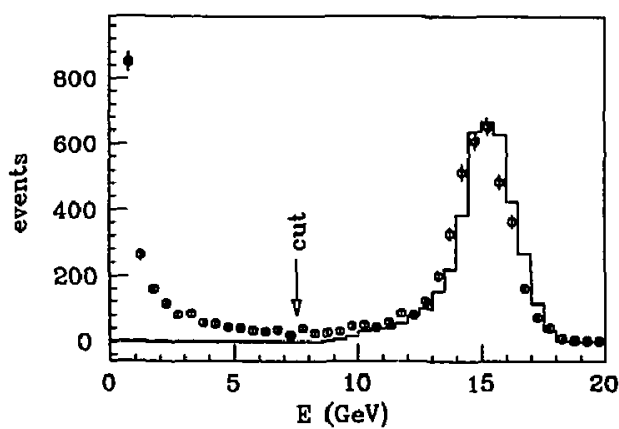

Figure 4.17. The luminosity counter energy deposit. The points are four-electron events, while the solid line is from Bhabha scattering.

13.45 GeV. Bhabha events do not suffer from much contamination of synchrotron radiation because they all have two back-to-back luminosity counter hits, while only a small fraction of the iuur-electron events have even one luminosity-counter hit. Therefore, the Bhabha events give some idea of what the low-energy side of the tag peak should look like. In Figure 4.17, a histogram of the messured Bhabhaelectron energy, multiplied by 0.95 , is plotted as a solid line over the points from the four-electron data.

Tagged events arc defined as those with one luminosity counter with energy greater than $7.5 \mathrm{GeV}$. Judging from the histogram of Fig. 4.17, we estimate that there is a maximur background from the tail due to noise of about 60 events and a maximum loss of about 50 events from the signal. There are 4051 entries above the cut (the histogram includes only about half of the data), so the conclusion is that the cut causes an uncertainty in the absolute normalization of no more than $\pm \mathbf{1 . 5 \%}$. However, the relative normalization of pions, muons, and electrons is not affected, because all of the tagged events are analyzed with the same cut. 


\subsubsection{Measured Kinematic Distributions}

Once the cut on luminosity counter energy has been chosen, the remainder of the analysis of tagged four-electron events proceeds almost the same as the untagged analysis. All other analysis cuts are the same, except that there is no cut on the total transverse momentum, $k_{\perp}$, for the tagged events. In this section we compase only the shapes of the measured distributions with those generated by the Vermaseren Monte Carlo program.

The acceptance of the luminosity counters is not well known, both because of inaccurate positioning and edge effects of electromagnetic showers, so there is some error in the Monte Carlo program due to integrating over an incorrect range of $Q^{2}$. In fact, the shapes of the invariant-mass and angular distributions for the two electrons observed in the central detector are not sensitive to that effect within the available statistical resolution. One distribution for the two electrons which is very sensitive to $Q^{2}$ is that of the total transverse momentum $k_{\perp}$. In fact, $k_{\perp}^{2}$ is approximately proportional to $Q^{2}$ for single-tag data. For untagged data, the $k_{\perp}$ distribution is dominated by the detector resolution, but that is not the case for the single-tag data, where most events have $k_{\perp}$ greater than $0.4 \mathrm{GeV}$.

Figure 4.18 shows the measured $k_{\perp}$ distribution compared with QED predictions for two assumptions about the luminosity-counter acceptance: (a) using the measured size and location of the face of the counter, and $(b)$ using the same geometry, but with the inner and outer edges moved radially inward by $0.9 \mathrm{~cm}$. One can see that the effective inner edge is almost a full centimeter inside the nominal edge of the counter face. This acceptance, which gives the best reproduction of the observed $k_{\perp}$ distribution, is used for the following analysis. There is a change of $14 \%$ in the cross section when going from the acceptance of Fig. $4.18 a$ to Fig. $4.18 b$, but the shapes of all distributions other than that of $k_{\perp}$ are not significantly affected.

To reproduce the lower edge of the $k_{\perp}$ distribution in detail would require a complete modeling of the luminosity-iounter response, which has not been

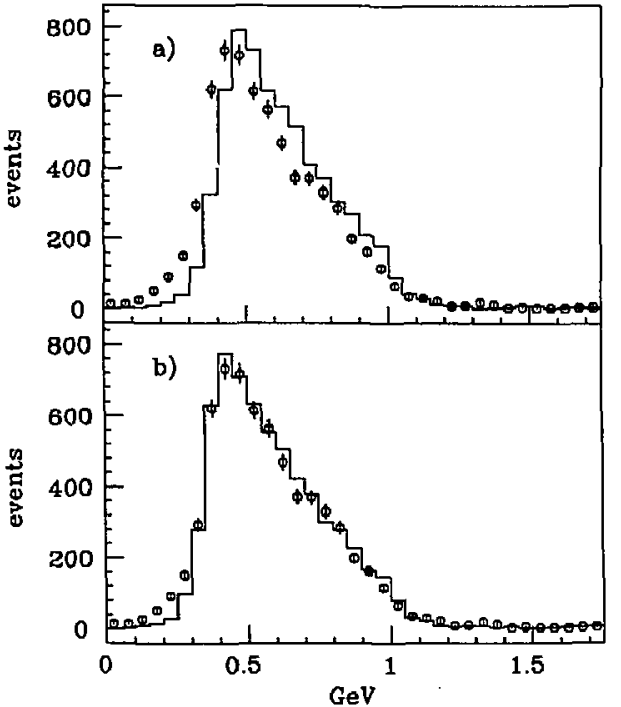

Figure 4.18. The $k_{\perp}$ distribution for tagged events. The points show the measured distribution of $k_{\perp}$ of two electrons in the central detector for tagged four-electron events. No corrections are made for detector effects. The solid lines in (a) and (b) show the QED prediction for two assumptions about the luminasity counter acceptance.

done. Also significant are measurement errors on the counter locations and QED radiative effects. It is important to emphasize that such a detailed analysis is not necessary for anything except to reproduce in detail the $k_{\perp}$ distribution. In particular, the relative normalization between events with electron pairs in the central detector and those with muon or hadron pairs in the central detector is not at all affected by the lack of detail in modeling the luminosity counters.

Figure 4.19 shows a comparison of the measured electron-pair invariant-mas 
distribution with the QED prediction from the Vermaseren Monte Carlo. The points are unfolded from the measured distribution, into an acceptance defined by $0.6 \leq W \leq 2.6 \mathrm{GeV}$ and $-0.6 \leq \cos \theta_{\mathrm{lab}} \leq 0.6$ for the pair of electrons in the central detector. Also, one of the scattered beam electrons must pass through a plane perpendicular to the beam, $282 \mathrm{~cm}$ from the interaction point, within the region between two hexagons concentric with the beam, where the inner hexagon has an inscribed radius of $6.82 \mathrm{~cm}$, and the outer hexagon has a circumscribed radius of $23.08 \mathrm{~cm}$. Figure 4.20 shows the comparison for the laboratory-system angular distribution of the two electrons observed in the central detector. Both distributions are in complete agreement with the QED prediction within the statistical error bars. The systematic errors are the same as for the untagged analysis, and in this case they are completely negligible compared with the statistical uncertainty.

\subsection{MEASUREMENT OF THE INTEgRated Luminosity}

$U_{p}$ to this point, the discussion has been limited to comparing the measured shapes of various distributions with QED predictions. It also is possible to measure absolute cross sections as long as there is an independent measurement available of the colliding-beam time-integrated luminosity (hereafter often referred to simply as luminosity or represented by the symbol $\mathcal{L}$ ). For DELCO, the best independent measurement of the luminosity comes from the process $e^{+} e^{-} \rightarrow \mu^{+} \mu^{-}$. In this section, the luminosity is measured using untagged events from the process $e^{+} e^{-} \rightarrow e^{+} e^{-} e^{+} e^{-}$and compared with the result from a measurement of $e^{+} e^{-} \rightarrow \mu^{+} \mu^{-}$.

The error in such a measurement is completely dominated by systematic effects, so it is of no advantage to choose cuts which allow in the maximum number of events. Nor is there any advantage even to use all of the data, so this discussion is restricted to the data accumulated during 1989 . Some systematic effects diffir slightly for other data, so it is more confusing to consider all of the

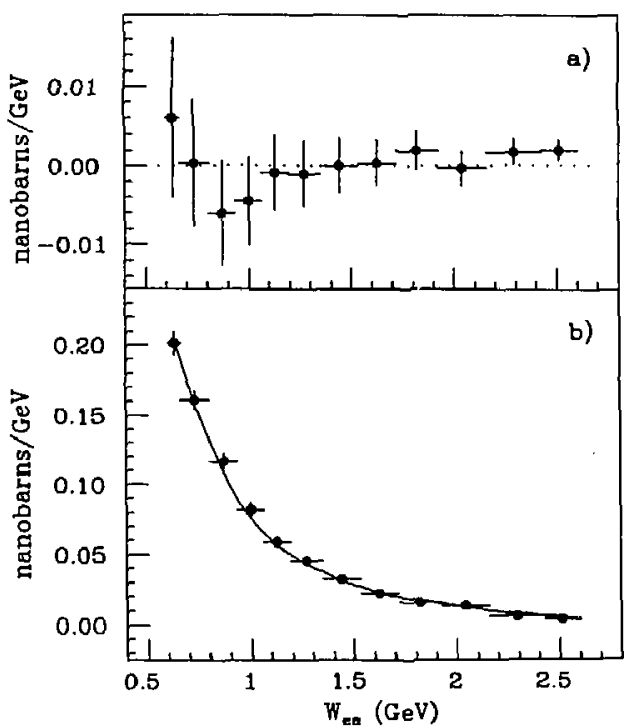

Figure 4.19. Electron-pair invariant mass for tagged events The data points are corrected for most detector effects. (a) The difference of the data and the QED prediction. (b) The QED prediction plotted as a smooth curve over the data.

data simultancously. The other data have been checked separately, and the results are consistent with those presented here. The 1989 data are specifically chosen because for them the most complete information is available on the $e^{+} e^{-} \rightarrow \mu^{+} \mu^{-}$ measurement.

Most of the cuts used are identical to those of Section 4.6. The most important exception is that here only those events are kept in which both tracks extrapolate to a point at least $1 \mathrm{~cm}$ inside of the edge of a shower counter, rather than just 


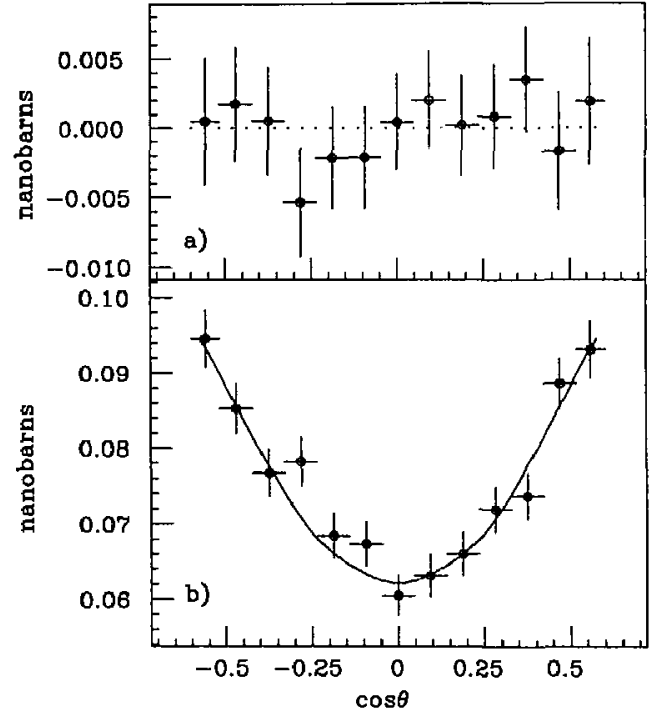

Figure 4.20. Angular distribution for e-pairs from tagged events. The data points are corrected for most detector effects. (a) The difference of the data and the QED prediction. (b) The QED prediction plotted as a smooth curve over the data.

inside. That is done in order to eliminate errors due to the detector survey or to the simulation by EGS of the edge effects. Also, the lower limit for $W$ is taken to be $0.7 \mathrm{GeV}$, rather than $0.6 \mathrm{GeV}$, in order to avoid the region of the worst trigger problems, and events with either track within the region $|\cos \theta|<0.05$ are discarded to avoid large corrections for the electron identification efficiency. The latter two changes are not essential, since the known corrections are adequate, but they are safe and do not harm the statistical accuracy.
After removing seven short runs for which no information is available from the Juminosity monitors, the number of events from data which pass all cuts is 10,380 . Several Monte Carlo tapes have been generated using the EPA Monte Carlo and a full simulation of the detector, including the use of EGS for the beam-pipe and drift chamber material and for the shower counter. The total generated luminosity is $26.5 \mathrm{pb}^{-1}$, which yields 4621 events after all analysis cuts are made.

Before quoting the measured luminosity, a number of small corrections must be made. They are due to various effects which are seen in the data but are not simulated in the Monte Carlo. The first concerns the requirement that exactly two tracks be seen in the inner drift chambers. Occasionally, extra tracks are produced either by noise hits in the drift chambers or by low momentum electrons from electromagnetic interactions in the beam pipe and drift chamber material. Such events can be found by considering all those with greater than two tracks and making pictures of those in which a subset of two tracks passes all of the analysis cuts. When that is done, $90 \%$ of the resulting events clearly are due to the hypothesized effects, and the remainder are consistent, but not certain. One finds that $0.7 \pm 0.2 \%$ of the signal is lost due to extra tracks being produced in the bearn pipe, compared with $0.65 \%$ in the Monte Carlo. Another $0.7 \%$ is lost due to tracks being produced from noise hits. That effect is not simulated at all, so a correction to the luminosity of $+0.7 \pm 0.3 \%$ is necessary.

The electron identification has not been simulated in the Monte Carlo. Excluding the bins for $-0.05<\cos \theta<0.05$, which are not included in this analysis, the efficiency found from Fig. 4.5 is 0.994 . Since both tracks in each event in the data must be identified, then that efficiency must be squared. To account for this effect, the luminosity is corrected by $+1.2 \pm 0.5 \%$.

The noise in the luminosity counters is not simulated in the Monte Carlo, while there is in the data a small loss of events due to noise greater than the $4 \mathrm{GeV}$ cut imposed to reject tagged events. That loss has been estimated by extrapolating the observed distribution, which yields a correction to the luminosity of $+1.0 \pm 1.0 \%$. 
As discussed in Section 3.1, the Monte Carlo appears to give a better momentum resolution than seen in data. That can cause less events to be cut in Monte Carlo by the $k_{\perp}$ cuts than in data and also may affect the lower cut on the invariant mass. To reproduce the $k_{\perp}$ distribution seen in data, a gaussian resolution function of width $0.04 \kappa$, where $\kappa$ is the measured track curvature, must be convolved with the curvature measured after the usual drift chamber simulation. When that is done, the number of Monte Carlo events passing all cuts drops to 4576, resulting in a correction to the luminosity of $+1.0 \pm 1.0 \%$

The cuts on the impact parameter and the average 2-position each reduce the data by only about $1 \%$, but even fewer events are cut from the Monte Carlo. There is no known background which can be contributing to the tails, so one must assume that the lost events are really part of the signal. These effects are accounted for by applying a correction of $+0.6 \pm 3.6 \%$ to the luminosity.

In addition to these correctiuns, there are a number of other sources of systematic error. The largest is due to uncertainty about the absolute momentum scale of the experiment, which is determined from measurements of the magnetic field. The scale is believed to be accurate to within $1 \%$. Since the invariant-mass spectrum falls like $1 / W^{3}$, and since the luminosity is proportional to the area under the curve and above the lower cut, then the resulting uncertainty in the luminosity is $\pm 2 \%$

A related uncertainty is duc to the EGS simulation of the beam pipe and inner drift ehamber material. One finds that the calculated effect of bremsstrahlung and other radiative processes in the bearn pipe and other material between the interaction point and the tracking chamber volume in which the momentum is measured is to lower the detection efficiency by $13 \%$. Only an insignificant part of that loss is due to such things as the cut on the track impact parameter. About $10 \%$ of the loss is due to worsening of the momentum resolution, resulting in more events being cut by the $k_{\perp}$ cuts. But the major effect is just $\approx$ downward shift of the average momentum, causing more events to fail the lower cut on

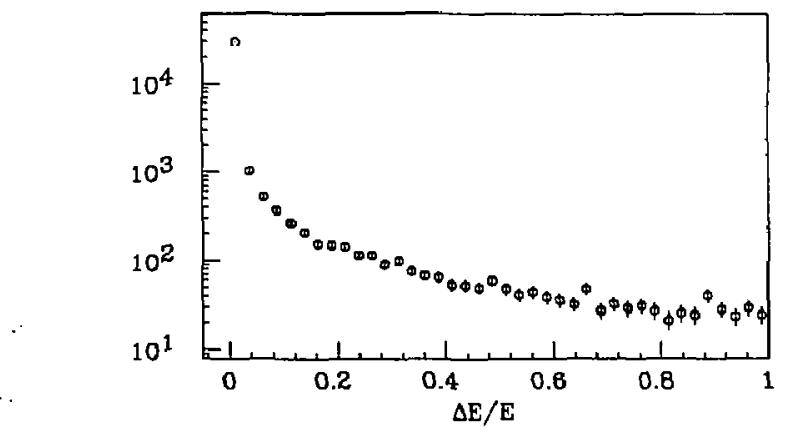

Figure 4.21. The fractional energy loss of electrons passing through 0.029 radiation lengths of aluminum between the beampipe vacuum and the innermost drift chamber gas volume, as calculated by the EGS Monte Carlo simulating 1083 data.

invariant mass. Figure 4.21 gives an idea of how that is possible. It shows the fraction of energy lost by electrons in the Monte Carlo when passing through the 0.0294 radiation lengths of aluminum between the beampipe vacuum and the gas volume of the inner drift chamber. Seven percent of the electrons lose greater than ten percent of their energy, and that is not including the additional 0.0054 radiation lengths of aluminum between the two inner drift chambers. We make a conservative assumption that the EGS calculation is good to at least $15 \%$. Since the correction is $13 \%$, then the systematic contribution to the uncertainty in the luminosity is only $\pm 2.0 \%$.

There is some uncertainty in the simulation of the drift chamber wire efficiency. After all of the other cuts have been made, the distributions of the numbers of bits look closely alike for data and Monte Carlo. In fact, the cut requiring at least 12 hits in the innes chambers reduces the data by $5.2 \%$ and the Monte Carlo by $6.3 \%$. It seems safe to assume that the uncertainty from this cut 
is no greater than $t 1 \%$.

There is an additional sourı: ol systematic en ror due to theoretical considerations. All calculations have been done only to leading order $\left(\alpha^{4}\right)$ in QED perturbation theory. The two multiperipheral graphs by far dominate the calculations, so it is important to consider possible radiative corrections to those graphs. Unfortunately, no calculations or Monte Carlo programs are available for the entire set of graphs for the order $\alpha^{5}$ corrections. Those corrections include radiation of real photons from any of the seven electron propagators of the leading order graphs, which gives amplitudes of order $e^{5}$, and all of the vertex corrections, electron self-energy corrections, and vacuum polarization corrections, which interfere with the leading order graphs to give $\alpha^{5}$ contributions in the cross section. Also, there are some entirely new graphs, with five-point functions, which should be included to be completely consistent, but those have been shown to contribute very little to the correction. ${ }^{35}$

The most thorough calculation of these corrections has been done for the related process $e^{+} e^{-} \rightarrow e^{+} e^{-} \mu^{+} \mu^{-} .^{36}$ Even that calculation neglects the graphs with five-point functions and those with three vertices on the muon line. $\dagger$ As long as no cuts are maule which depend heavily on the energies of the scattered beam electrons or on the total neutral energy, which is the case for this analysis, then the differential cross section at each value of $W_{\mu^{+} \mu^{-}}$is found to increase by less than $1 \%$ when the corrections are introduced. It is not surprising that the corrections are small, because the $Q^{2}$ of the photons are very small, and the momentum transfer is only the order of one GeV. Due to the impracticality of doing the complete calculation, we do not apply any radiative correction, but simply assume that the resulting error is $\pm 1 \%$.

Table 4.5 summarizes all of the luminosity corrections and systematic errors. After adding the systematic errors in quadrature with each other and the $\pm 1.8 \%$

\footnotetext{
† Twenty-aix diagrams are included, so even with the approximations which are made, the calculation is far from simple.
}

statistical uncertainty, we arrive at the result:

$$
\mathcal{L}^{18^{8} 3}=62.2 \pm 2.5 \mathrm{pb}^{-1}
$$

Carrying through the exercise for the full data set taken with the isobutane Cerenkov radiator gives a total integrated luminosity of $122.4 \pm 4.5 \mathrm{pb}^{-1}$.

Table 4.5- List of the sources of error in the luminosity measurement from untagged four-electron data and the associated corrections.

\begin{tabular}{|c|c|}
\hline Source of Error & Percent Correction \\
\hline two-track cut & $+0.7 \pm 0.3$ \\
\hline electron identification & $+1.2 \pm 0.5$ \\
\hline luminosity-counter cut & $+1.0 \pm 1.0$ \\
\hline momentum resolution & $+1.0 \pm 1.0$ \\
\hline impact parameter and $z$ & $+0.6 \pm 0.6$ \\
\hline momentum scale & \pm 2.0 \\
\hline EGS simulation & \pm 2.0 \\
\hline wire efficiency & \pm 1.0 \\
\hline radiative effects & \pm 1.0 \\
\hline statistical uncertainty & \pm 1.8 \\
\hline
\end{tabular}

For each of the runs which have been analyzed, there is a luminosity measurement from the small-angle Juminosity monitors. Those measu rements are not absolute in themseives because of difficulty with understanding the luminosity monitor acceptance. But that acceptance is constant over each run-block, so the measurements $d 0$ give the relative luminosity for each run. Using them ard applying a correction ditermined from the measurement of $e^{+} e^{-} \rightarrow \mu^{+} \mu^{-}, 37$ one find that the luminosity to compare with Eqn. 4.7 is $\mathfrak{L}_{e^{+} e^{--} \rightarrow \mu^{\top} \mu^{-}}^{18_{3}}=$ 
$69.8 \pm 3.5 \mathrm{pb}^{-1}$. If the error estimates for these two results are added in quadrature, then it appears that they disagree by 1.8 standard deviations. However, so far all the contributions to the error estimates have been added in quadrature, and that almost certainly gives an underestimate of the error, even though the individual contributions listed in Table 4.5 are conservative estimates. Adding all the errors in Table 4.5 linearly $(\approx 11 \%)$ is certain to give $\alpha \Omega$ overestimate of the error but would bring the two results into good agreement. It also is interesting that if it is true that the experimental momentum scale is in error by $-2 \%$, as indicated by the measurement of $K_{0} \rightarrow \pi^{+} \pi^{-m}$ (see Section 3.1), then the result in Eqn. 4.7 must change by $+4 \%$, which would bring it intc reasonable agreement with the muonpair measurement. These difficulties only underscore the important ract that when using electron pairs to normalize the pion-pair sample, most of the errore listed in Table 4.5 no longer contribute. In particular, any error in the momentum scale cancels. Obviously, none of the errors inherent in the measurement of $e^{+} e^{-} \rightarrow \mu^{+} \mu^{-}$contribute in that case either.

\subsection{INTEGRATED LUMINOSITY FROM TAGGED EVENTS}

The measurement of the luminosity can be repeated with tagged events, although then the error is dominated by the lack cf knowledge of the luminosity counter acceptance. The analysis of tagged four-electron events is repeated with the same changes that were made to the untagged analysis: the barrel shower counter acceptance is restricted, the center of the detector in $\cos \theta$ is cut out, and the lower limit on the $e^{+} e^{-}$invariant mass is moved up to $0.7 \mathrm{GeV}$. For the 1989 data, 1826 events pass all the cuts.

Most of the corrections which apply to the untagged analysis apply here as well. One exception is that because no cut is made on $k_{\perp}$, the uncertainty in the momentum resolution has very little effect. Also, the cut on luminosity counter energy obviously is changed, and it is appropriate to use the systematic error estimated in Section 4.9.1. Table 4.6 gives a list of the necessary corrections and errors. Note that the error due to the luminosity counter acceptance is not included.

Table 4.6. List of the sources of error in the luminosity mesaurement from tagged four-electron data and the associated corrections.

\begin{tabular}{|c|c|}
\hline Source of Error & Percent Correction \\
\hline two-track cut & $+0.7 \pm 0.3$ \\
\hline electron idenification & $+1.2 \pm 0.5$ \\
\hline momentum resolution & $-0.4 \pm 0.4$ \\
\hline impact parameter and $z$ & $+0.6 \pm 0.6$ \\
\hline luminosity-counter cut & \pm 1.5 \\
\hline momentum scale & \pm 2.0 \\
\hline EGS simulation & \pm 2.0 \\
\hline wire efficiency & \pm 1.0 \\
\hline radiative effects & \pm 1.0 \\
\hline statistical uncertainty & \pm 3.5 \\
\hline
\end{tabular}

When the measured location and size of the face of the luminosity counters is assumed for the acceptance, the measured luminosity is $76.3 \pm 3.7 \mathrm{pt}^{-1}$. However, we have seen that it does not serm reasonable to assume that acceptance. When, instead, the acceptance is used which gives the best fit to the measured distribution of $k_{\perp}$ of the two electrons in the central detector, then the measured luminosity changes to $\mathcal{L}_{\text {tag }}=65.4 \pm 3.5 \mathrm{pb}^{-1}$. The latter result agrees with the untagged measurement. In spite of the uncertainty about the luminosity counter aceeptance, we have seen that everything is in agreement if a reasonable assumption is made about that acceptance. The matter will not be puraned any further, because it is not relevant to the use that will be made of togged events in the remainder of the analysis. 


\section{Measuring the Pion-Pair Spoctrum}

After electron pairs have been removed from the two-track data sample, most of the remaining events are muon pairs and pion pairs produced by $\gamma \gamma$ interactions. DELCO has no capability of distinguishing between pions and muons, but the majority of the events are in fact muon pairs. It is essential to be able to predict their contribution to all of the distributions in order to subtract it. The QED Monte Carlo programs are used with complete confidence to generate the theoretical spectrum of muon pairs. The only remaining problems are to simulate the detector response and normalize the theoretical calculation.

Fortunately, the detector response is easy to understand for muons, compared with electrons or hadrons. In particular, the only trigger effects which must be understood are the geometric limits of the shower counters and the effect of clectromagnetic energy loss. The latter is easy to prediet for massive particles and simply results in a sharp momentum cutoff, at about $180 \mathrm{MeV}$ for muons.

The normalization requires a knowledge of the Iuminosity of the colliding beams. That can be predicted from knowledge of the storage ring optics and the measured beam intensity, but not to sufficient accuracy to be of any use to most physics measurements. What generally is done instead is to measure a physics process, resulting from the beam collision, which can be predicted theoretically to the desired accuracy. The obvious candidates are simple QED processes. For otudies of $e^{+} e^{-}$annihilation, Bhabha scattering and muon-pair production commonly are used. Those processes, however, result in electrons or muons with energies close to $14.5 \mathrm{GeV}$ at $P E P$, compared with energies of less than $0.5 \mathrm{GeV}$ for most particles resulting from two-photon production. As a result, the systematic effects involved in measuring the two-photon events are completely different from those encountered with Bhabha scattering or muon-pair production, and one can expect uncertainties when extrapolating from high to low energy which will greatly dominate all other errors involved in the pion-pair measurement.
DELCO has the unique ability to use the $e^{+} e^{-} \rightarrow e^{+} e^{-} e^{+} e^{-}$process for normalization. The results of Chapter 4 demonstrate complete agreement of measurements of several kinematic distributions from this process with QED predictions. One general advantage gained is the similarity of the energy and angular distributions of the electron final state to those of the processes which require normalization. That is especially true for normalization of the process $e^{+} e^{-} \rightarrow e^{+} e^{-} \mu^{+} \mu^{-}$, since when well above the $\mu^{+} \mu^{-}$threshold, the cross section $i$, alrnost ident $k .+1$ eo that of the four-electron process. Another important advantage is that the initial state is the anme for all proceases considered.t

As a result, there are many specific sources of systematic error in the normalization which cancel. If the process $e^{+} e^{-} \rightarrow \mu^{+} \mu^{-}$were used for normalization, there would be all of the systematic errors associated with the measurement of the high energy muon pairs plus all of the systematic errors listed in Section 4.10 for the measurement of the luminosity from untagged electron pairs. Instead, one findo that it is not necessary to understand completely many effects. For example, radiative effects related to the $e^{+} e^{-} y$ wertices largely cancel. Plus, for normalization of the muon-pair channel, any final-state radiative effects differ only by small mass-dependent terms. Any errors in the knowledge of the detector acceptance cancel, and the efficiencies of the various noise cuts and tracking cuts need not be well known because they are almost the same for all channels. There is no need to worry about the momentum scale being slightly wrong, either, because the energy spectra for all of the processes behavi almost the same and thus would be affected by the same amount. In summary, using a two-photon channel for normalization greatly reduces the error estimate and decreases the likelihood of additional errors from effects not considered.

of course, not all systematic effects cancel, but those which remain are small and relatively easy to pinpoint and understand. They result from differing

T That is true to the extent that only the multiperipheral diagrame contribute to the QED crove sections. Refer to Section 4.5 for a more complete discussion of this point. 
responses to electrons and muons of some parts of the detector. The most important problems involve the shower counterg, and hence the trigger. First of all, the latch efficiency depends on the particle type. It has been measured $\dagger$ for electrons and is easy to predict for muons, so the necessary corrections can be made. Second, the geometric acceptance of the trigger is larger for electrons than muons because of the lateral spread of electromagnetic showers. That problem can be avoided by doing the normalization with only those events far from the shower-counter edge and then extrapolating to the full acceptance. Also, there is a small amount of material between the beam-beam interaction point and the drift chambers where the momenta are measured. As a result, electrons can lose a significant amount of energy through bremsstrahlung before being measured, while that is not possible for muons and pions. That effect can be corrected for by use of the EGS Monte Carlo. The only other significant problem is with the different efficiencies for particle identification by the Cerenkov counter. Those are measured from the data and may be corrected for with minimal error. The remaino - of this chapter concentrates on understanding these corrections and applying them to obtain the subtracted pion spectrum.

\section{1 Rejection of Four-Elegthon Events}

Events with no electrons in the central detector are easily selected by requiring that there be essentially no signal from any Cerenkov counter associated with a track. Since both of the muons or pions are required to pass through the acceptance region of the Cerenkov system, then the rejection of electrons is close to $100 \%$. In fact, a simple calculation using the efficiencies in Table 4.1 quickly shows that the electron background is completely negligible. There is, however, a small loss of efficiency due to random noise in the Cerenkov ccunters at the one-photoelectron level. Such signals must be rejected as possibly being from electrons if they have time residuals close to zero.

f Refer to Section 4.5 for a diseussion of the electron showar counter latch efficiency
Figure 5.1 shows distributions of the total in-time raw Cerenkov pulse height associated with each track, where in-time means those cells with time residuals less than $1.65 \mathrm{~ns}$. The first histogram includes all particles, while the second includes only particles for which the opposing one has been unambiguously identified as a muon or pion. $\dagger$ Such identification is accomplished by accepting only particles which pass through the highly efficient regions of the Cerenkov system with momenta greater than $0.3 \mathrm{GeV}$ and have an associated raw pulse height of less than 0.1 phatoelectrons. The noise peak in the pion-muon data overlaps with the electron data, so a cut at $\mathbf{0 . 2}$ photoelectrons is chosen such that it is rejected. Figure 5.2 shows distributions of the total out-of-time raw pulse height associated with each track. Note that a single track may have entries in both figures 5.1 and 5.2, so the efficiencies cannot be simply read from the histograms, though they do give a reasonable first entimate. The cut on the out-of-time pulse height is placed above the noise peak at 1.5 photoelectrons.

There are a few "muona" with quite large pulse heights. They are not likely actually to be electrons, but probably are pions passing through Cerenkov cells which have otherwise unseen photon conversions within. In that case they would have to be considered as background events, not as contributions to the identification inefficiency. To do this analysis, all possible cutst have been included to reject background from processes with multiplicities greater than two, except for those which could bias the identification itself. The very low incidence of overlap with photon convergions is evidence in itaelf of the resulting cleanliness of the data, and, in fact, there are few enough ouch events that one need not be concerned about them giving a significant error to the measured identification efficiency. With that in mind, the efficiency, as determined from those particles whose partner has been unambiguously identified as a muon or pion, is $99.5 \%$ for each track and has no angular or momentum dependence.

t Thero are, of course, sto a few kaons and protons, but the distinction is of no relevence here. $\$$ The cuts are listed in Section $\mathbf{5 . 2}$. 


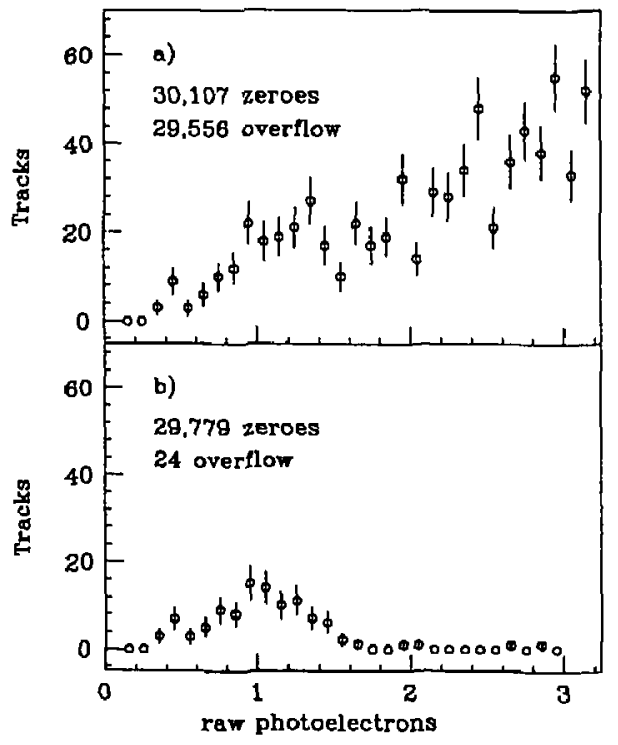

Figure 5.1. The Cerenkov in-time raw pulse height distribution for (a) electrons and muons and (b) for muons only.

5.2 ANalysis CUTS For THE UNTAgged $\pi^{+} \pi^{-}$Signal

The analyais cuts used on the untagged sample of pion and muon pairs are much the same as those used on electron pairs, with the addition of several cuts necessary for reducing background in the hadronic channel. The kinematic cuts are unchanged except that pion, rather than electron, masses are used for computing the two-particle invariant mass. There is only a slight change in the definition of the angular acceptance. Recall that for electrons, the $\phi$ acceptance is defined by requiring the measured tracks to pass through where the shower-counter modules

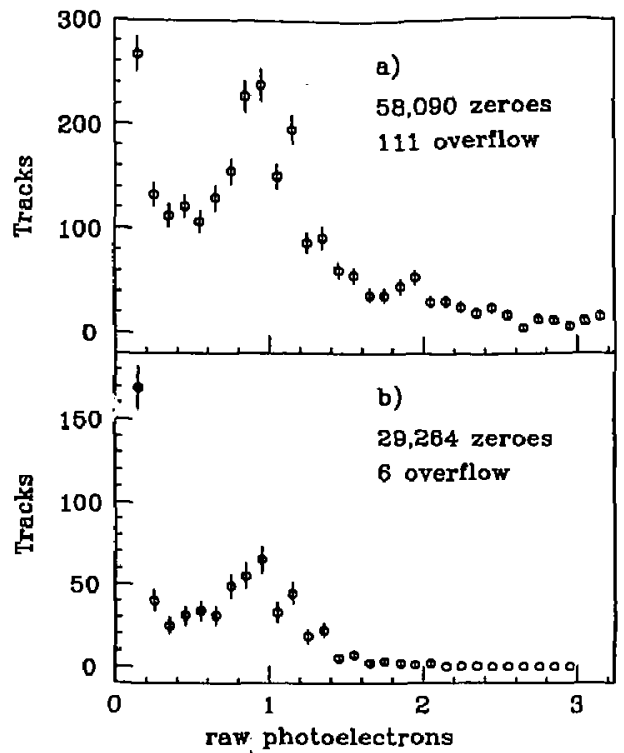

Figure 5.2. The Cerenkov out-of-time saw pulse height distribution for (a) electrons and muons and (b) for muons only.

are believed to be. However, the trigger for muon and pion pairs already requires that both tracks latch a shower counter, a requirement which is enforced in the analysis by ensuring that no other incidental triggers (from noise, for example) are allowed to contribute events in which one of the tracks has failed to latch a shower counter. An additional cut on the tracking and survey information is not made because it would only confuse the understanding of the pion detection efficiency, due to lack of understanding of the effect of nuclear scattering on the extrapolation of the measured track. This point is discussed further in Chapter 6. 
The cuts which are used in the electron analysis for selecting events with well measured tracks are used here without change. However, many of them also serve to reject background processes, none of which are present in the electron-pair sample. All of the remaining cuts serve only to reject background.

The first background to consider comes from cosmic-ray muons. If they happen to pass near the detector center within about $20 \mathrm{~ns}$ of the beam crossing, then they can mimic colinear pairs of muons produced by the beams. Simply rejecting pairs which are almost colinear is not a desirable solution because pairs from untagged events already are nearly colinear in the transverse plane, and such a cut would be applied just where the detection acceptance is a maximum. The $0.5 \mathrm{~cm}$ impact-parameter cut and the cut on the average $z$ of the tracks near the origin immediately remove a large fraction of the cosmic-ray events present on the data tapes. The rest can be tagged effectively by measuring with the time-of-fight counters and shower counters the time required for the muon to pass from one side of the detector to the other.

The timing information is considered only if the angle between the two tracks is greater than 168.5 degrees. A time-of-fight counter is used on a given side if one is hit and has times at both ends which are consistent within three standard deviations. $\dagger$ Otherwise, a shower counter is used if one is found which passes the Bame requirements. Only $0.5 \%$ of the events considered have no usable timing information; they merely are assumed not to be cosmic rays. Figure 5.3 shows the distribution of the time difference, made before most of the analysis cuts, including vertex cuts, are applied. The separation of cosmic rays from the signal is clear; the cut is chosen to reject all events with a time difference greater than $8.0 \mathrm{~ns}$.

Another background source is scattering of beam electrons on residual gas inside the beam pipe. Such events have a flat distribution of the $z$ position of their origin, while the beam-beam interactions occur within about $3 \mathrm{~cm}$ of the detector center. Figure 5.4 shows the distribution of the average $z$ of the track

† Refer to Chapter 9 for a detailed discusaion of the time-of-fight analysis.

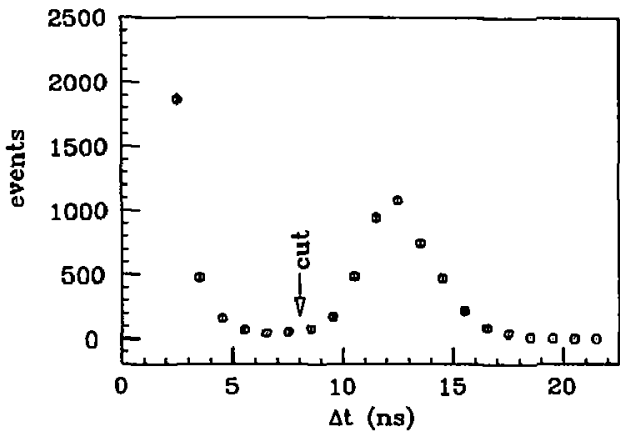

Figure 5.3. Cosmic-ray rejection. $\Delta t$ is the difference in time of a timeof-light or shower counter associated with one track and that associated with the second track. The total number of events entered is 58,940 . 51912 fall in the bins from zero to two nanoseconds. Events which have an angle between tracks less than 168.5 degrees are entered with a zero time difference.

points closest to the beam line in each event. All other analysls cuts have been made-in particular, cosmic rays have been rejected. A cut rejecting evento with $|z|>4.0 \mathrm{~cm}$ eliminates the flat tail without cutting appreciably into the signal. The tail itself gives an upper limit on the contamination from beam-gas scattering in the signal region, because the detection efficiency for tracks a few centimeters outside of that region is approximately what it is within.

The remaining background is from genuine beam related events, either by $\gamma \gamma$ production or $e^{+} e^{-}$annihilation. Many hadronic final states can mimic a pair of pions if all other particles in the final state escape detection. The analysis must rely on the cut on $k_{\perp}$ to reduce such background to a low level. The tracking resulution does not allow a cut on $k_{\perp}$ any tighter than what has been used for the electron analysis without causing an unacceptable loss of the signal. Therefore, some background remains, and its level must be estimated. 


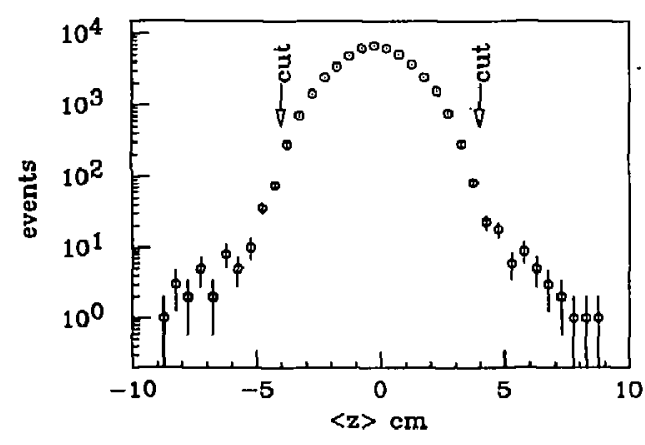

Figure 5.4. The $z$ position of the interaction point. It is calculated by averaging the $z$ coordinates of the points of closest approach of the tracks to the beam-line. Only events with two non-electron tracks which have passed all of the $\pi^{+} \pi^{-}$analysis cuts, except for the cut on $\bar{z}$, are used.

It is important that if any extra particles are detected by any means that the event be rejected. Therefore, cuta are made on the pulse heights of shower counters and Cerenkov counters which are not associated with either of the two tracks. The total pulse height of the pole-tip counters must be less that $0.1 \mathrm{GeV}$, and the pulse height of the barrel shower counters, summed over all counters not hit by a track and not adjacent to one hit by a track, must be less that $0.15 \mathrm{GeV}$. Any event with a Cerenkov counter which has a time residual less than $1.65 \mathrm{~ns}$ and a ravy pulse height greater than 3.0 photoelectrons is rejected. Finally, a search is made for track stubs in the planar drift chambers. If a stub is found with at least one stereo wire, two axial wires, and four total wires and has a $\chi^{2}$ per degree of freedom less than 4.0 from fitting a straight line to the hits, then the event is rejected, unless the stub is found to be sharing wires with one of the two tracks or using any wires adjacent to one used by a track.
These noise cuts are designed primarily to detect photons, which may leave a signal in a shower counter or convert in material in the Cerenkov counters or planar drift chambers. They also serve to reject events in which a charged particle has been missed by the tracking system but still hits a shower counter. The cut values have been tuned and tested by looking at single-event displays as well ao histograms. Their effect on the actual signal can be checked by applying the same cuts, except for cuts on Cerenkov pulse height, to clectron pairs, since there is no significant background to the four-electron channel. After all other analysis cuts have been made, the noise cuts reduce the $e^{+} e^{-}$sample by only $1.2 \%$.

The analysis commences with summary tapes including two-track events with no cuts on particle ty pe, since the electron-pairs must also be present to provide the normalization. A total of 398,936 cvents are input, and the trigger requirement eliminates 2,969. Before separating the particle types, most of the other cuts are made. Those cuts and their effects are listed in Table 5.1. Of the 138,590 remaining events, 61,798 are positively identified as electron pairs and 75,094 as pairs of nonelectrons. The 1698 events which fail the cuts for both categories are rejected.

Considering now only the category of non-electron events, the requirement that each track at least pass near to a shower counter rejects 1180 events. The cuts on planar drift chamber stubs and barrel-shower and Cerenkov noise reject 1413 events, and requiring both tracks to latch a shower counter rejects 1670 events. The latter cut is made in order to ensure that both tracks are vithin the barrel-show ar acceptance and that the trigger for the event is not from some random source. That is necessary if the acceptance and the trigger response are to be understood. Finally, 72 events are rejected because the two tracks are of the same charge. The result is a sample of 70,759 events, including muon and pion pairs with a small fraction of background from kaon and proton pair production and other hadronic processes. To produce the p on-pair spectra, the corresponding muon-pair spectra must be calculated and subtracted, and the level of the other backgrounds must be estimated. 
Table 5.1. List of some of the cuts for the $\pi^{+} \pi^{-}$analysis. The remainder are listed in the text. The fourth and fifth columns give respectively how many events would be rejected by the cut before all other cuts and how many would be rejected after all others.

\begin{tabular}{|c|c|c|r|r|r|}
\hline Quantity & $\begin{array}{c}\text { Lower } \\
\text { limit }\end{array}$ & $\begin{array}{c}\text { Upper } \\
\text { limit }\end{array}$ & $\begin{array}{c}\text { Failures } \\
\text { when first }\end{array}$ & $\begin{array}{c}\text { Failures } \\
\text { when last }\end{array}$ & $\begin{array}{c}\text { Failures } \\
\text { in order }\end{array}$ \\
\hline poletip $E$ & 0 & $0.1 \mathrm{GeV}$ & 18389 & 3112 & 18389 \\
\hline inner DC hits & 12 & 16 & 21938 & 3779 & 18892 \\
\hline fit errors & 0 & 0 & 3537 & 92 & 822 \\
\hline $\bar{z}$ & $-4.0 \mathrm{~cm}$ & $4.0 \mathrm{~cm}$ & 6149 & 427 & 2996 \\
\hline impact & & & & & \\
\hline parameter & 0 & $0.5 \mathrm{~cm}$ & 24786 & 1370 & 11327 \\
\hline cos $\theta$ & -0.6 & 0.6 & 74593 & 33699 & 62128 \\
\hline$W_{\pi \pi}$ & $0.6 \mathrm{GeV}$ & $2.0 \mathrm{GeV}$ & 156867 & 24206 & 114480 \\
\hline$k_{\perp} / W_{\pi \pi}$ & 0 & 0.2 & 96288 & 27134 & 27140 \\
\hline$k_{\perp}$ & 0 & $0.3 \mathrm{GeV}$ & 23080 & 241 & 250 \\
\hline MAX $\left(p_{t}^{1}, p_{t}^{2}\right)$ & $0.25 \mathrm{GeV}$ & $\infty$ & 85606 & 782 & 783 \\
\hline $\operatorname{cosmic}$ & 0 & 0 & 4626 & 170 & 170 \\
\hline
\end{tabular}

\subsection{Subtraction of THE $\mu^{+} \mu^{-}$Background}

Once the proper normalization has been determined, it is straightforward to calculate how many muons are present in each bin of any distribution. QED is used to calculate the physical distributions, by means of the Vermaseren Monte Carlo, or in the untagged case, equally well by the EPA Monte Carlo. $\dagger$ We continue here with the untagged sample, but the methods also apply to the tagged sample.

f For the case of muon-pair production, well as the four-electron final state, the EPA program has been checked to give reaulta identical to those of the Vermaseren program when an untagged analyais is made.
Detector effects are especiaily simple to simulate for muons. The identification efficiency has no kinematic dependence within the fiducial volume, and the shower counter latch efficiency is affected only by electromagnetic range-out. The amount of material traversed by muons going in various directions does not vary greatly, and range fluctuations are small (they actually are neglected in the Monte Carlo), so the result of the electromagnetic effects is simply a rather sharp cutoff where the efficiency drops to zero below a particular value of the initial momentum. This cutoff can be predicted by using the full simulation of the detector, including the program for detailed yimulation of the shower counter geometry and materials.

To check the protram, the calculation is made for a combination of tagged Monte Carlo muon pairs and pion pairs in approximately the froportion found in the data. Only tracks which pass through a counter well away from the edge are used, so the histogram shows the probability of the particle actually stopping in the material before the second layer of scintillator. Tagged events are used because their LS trigget, which requires a coincidence of a luminosity counter and a barrel shower counter, allows events to be seen in which one particle fails to latch the shower counter. Therefore, the Monte Carlo can be compared directly with the data as long as the trigger is simulated. Figure 5.5 shows that the sharp edge seen in the Monte Carlo agrees well with that seen in the data, although, as expected, the data show an additional and more slowly varying inefficiency due to nuclear interactions of pions.

Electron pairs are used to provide the normalization, but care must be exercised to correct for differences in acceptance of the trigger for electrons and muons. It is of no help simply to require the same trigger for the two types, because the $2 \mathrm{~S}$ trigger has a dramatically different response for electrone as compared to muons. Instead, electron events with only a KS trigger still are accepted, and the inefficiency caused by ranging out of electromagnetic showers is corrected for by using the measurements of Chapter 4. Likewise, the effect of range-out on the muons is corrected for by using the curve in Fig. 5.5. There is the additional 


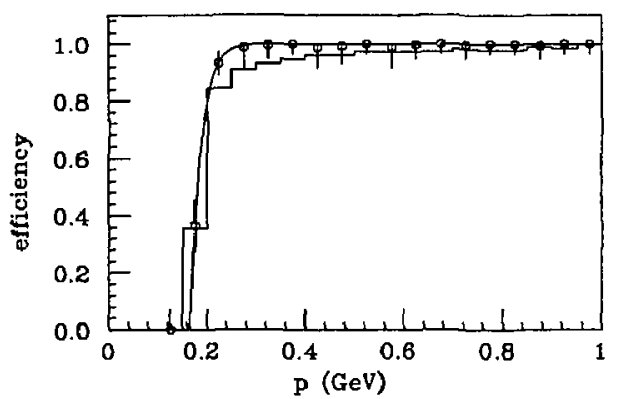

Figure 5.5. The Monte Carlo shower counter latch efficiency for a mixture of tagged pion and muon pairs (points) is compared with that measured from tagged data (solid histogram). The Monte Carlo does not include nuclear interactions. The smooth curve represents Eqn. 4.2 with the parameters $A=1, p_{0}=0.177$, and $r=0.0219$.

problem of edge effects, however. Electrons have a larger acceptance in the shower counters than do muons, because showers in the aluminum surrounding the counters cin spread into the scintillator. To some extent, that is simulated by EGS, but it is best not to rely on a complicated Monte Carlo calculation. It also is not a good idea to try to use the tracking information to specify when a particle is within the shower counter edge, because of inherent tracking and survey errors. The approach that is taken is to define a muon or pion as being within the acceptance if it latches a shower counter. That means that the few events must be discarded which rely on triggers from some source besides the two shower counters actually hit by the muons or pions. Electron pairs, on the other hand, are kept only if both tracks are determined by tracking to pass through a shower counter at least $\mathbf{4} \mathrm{cm}$ from the edge. That gives a large enough margin for error that the survey and tracking errors do not affect the acceptance (see Fig. 5.6). The only remaining problem is to determine the difference in geometric acceptance for muon

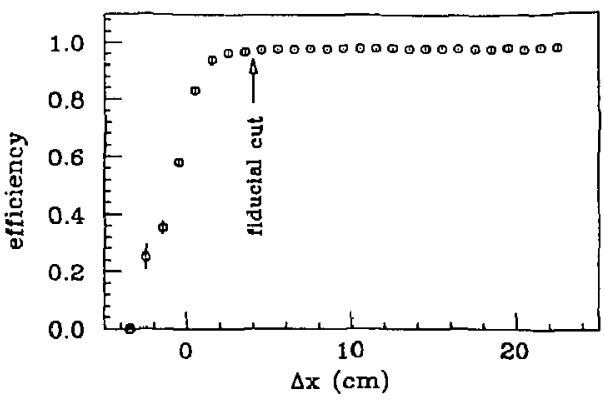

Figure 5.6. The resolution of the shower-counter edge for electrons, The efficiency for the first layer of the shower counter to latch is plotted as a function of the distance from the nearest edge of the counter.

pairs between the case where the 4-cm cut is made as for the electron pairs and the case where only two latches are required. After making all of the analysis cuts, the muon-pair Monte Carlo with full detector simulation gives an acceptance ratio of $1.469 \pm 0.020$, while the non-electron data gives $1.450 \pm 0.007$. The fact that the two agree shows that the width of the counters is correct in the Monte Carlo and that there is not much of an edge effect from pion-nucleus interactions; the more precise value is used for the analysis. Note that the corresponding ratio for electron pairs is found to be $1.493 \pm 0.010$. Thus the edge effect from electromagnetic showers could cause as much as a $3 \%$ increase in acceptance

Of the 61,798 elcctron pairs, 6455 are rejected because one track does not have an associated shower counter. The cuts on planar drift chamber stubs and barrelshower and Ćerenkov counter noise reject 678 events, and 20,366 are rejected in order to satisfy the $4-\mathrm{cm}$ fiducial cut in the shower counters for each track. Of the remaining events, 14 hase two electrons of the same charge and are rejected. Since the final $\pi^{+} \pi^{-}$invariant-mass spectrum must be calculated by assuming pion masses for both particles, then the cuts should be made for all particle types 
by assuming pion masses. However, the cut of $W_{\pi \pi} \geq 0.6 \mathrm{GeV}$ is lower than the invariant-mass cut made in the analysis of Chapter 4 . For that reason, the region between $W_{\pi \pi}=0.6 \mathrm{GeV}$ and $W_{\pi \pi}=0.7 \mathrm{GeV}$ cannot reliably be corrected for systematic effects. Only the 23,645 events in the range from $0.7 \mathrm{GeV}$ to $2.0 \mathrm{GeV}$ are used for normalization.

To proceed, the same number of Monte Carlo events are generated, and all detector effects are included as in Chapter 4, including the trigger and identification efficiencies. One finds that the data represent an effective luminosity of $64.07 \pm 0.42 \mathrm{pb}^{-1}$. It is not the true beam lurrinosity because not all inefficiencies of the detector have been included in the calculation. In particular, many with only a $\phi$ dependence are neglected. Effects which differ between electrons and muons, such as the electron identification efficiency and the electron shower counter latch efficiency, have been included. The one exception is the effect of the beam-pipe and drift-chamber material.

We have seen that the material in the shower counters and in front of them affects the normalization by reducing the trigger efficiency for electrons relative to muons. In that regard, the material in the beam pipe and the inner drift chambers is insignificant. However, the innermost material that the particles must pass through is unjque in that it affects the particles before their momenta are measured. There are two layers of aluminum which are important in this respect: The first is the material of the beam pipe and the inner wall of the innermost drift chamber, comprising 0.029 radiation lengths for $1982 / 1989$ data and 0.013 radiation lengthe for $198 \mathrm{~d}$ data, and the second is the material between the two inner drift chambers, comprising 0.0054 radiation lengths. The material between the inner and outer drift chambers is not relevant because the outer drift chambers play little part in the momentum measurement for low-momentum tracks.

Electromagnetic interactions of electrons in the aluminum cause several losses in addition to those incurred by muons. In about $0.3 \%$ (in 1981) to $0.6 \%$ (in $1988 / 1989$ ) of the events, extra electron tracks are produced with high enough . momentum to be found by pattern recognition. Energy loss from bremsstrahlung causes several other effects. The impact parameter distribution is broadened slightly, as is the distribution of $k_{\perp}$, resulting in the loss of an additional couple of percent of the events. But by far the biggest effect is simply due to a slight shift in ine energy acale caused by the average energy loss incurred by electrong before reaching the region where their momenta are measured. Of all of the cuts made, most are placed well out in the tails of distributions and hence are not very sensitive to any effects. The exceptions are the cuts defining the angular acceptance, which are not affected by the energy loss, and the cut on invariant mass, which is very sensitive to any shift in the energy scale.

The EGS Monte Carlo is used to determine the effect of the material on electrons. Figure 4.21 shows the fractional energy loss of electrons in the firat layer of aluminum for the 1983 data. The average loss is $3.3 \%$, and $7 \%$ of the electrons lose greater than $10 \%$ of their energy. Since the invariant-mass spectrum falls as $1 / W^{3}$, a downward shift by $x \%$ in the energy scale will decrease by $2 x \%$ the number of events above any given cut, so one can see that the bremsstrahlung must have a drastic effect on the normalization.

The bremsstrahlung is found not to affect noticeably the shape of the invariant-mass distribution, so all that is necessary is to calculate a constant correction factor for each of the data sets. That is done by taking the ratio of the efficiency found from the Monte Carlo with full detector simulation, including EGS where necessary, for muon pairs with respect to electron pairs. The results are $0.889 \pm 0.008$ for $1982 / 1989$ data and $0.946 \pm 0.009$ for the 1981 data, or $0.908 \pm 0.008$ overall.

There also is some unknown systematic error from the EGS calculation. EGS, along with the rest of the detector simulation software, does predict correctly the number of extra tracks produced by electromagnetic interactions, which is $0.7 \pm 0.2 \%$ in the $1982 / 1989$ data. It also has been shown to predict correctly the latch efficiency in the shower counters, but of these two checks, the first 
is not very significant statistically and the second is not directly related to the beampipe. However, EGS has generally been found to be reliable, and calculating the energy loss of an electron passing through a thin sheet of aluminum is a relatively simple application of the program. Therefore, it is reasonable to assume that it gets the correction right to at least $15 \%$, and since the correction itself is about $10 \%$, then the resulting error in the normalization is about $1.5 \%$. Including this correction, along with systematic errors from the trigger and electron-identification corrections and the statistical error from the sample of fourelectron events, results in a corrected effective luminosity for the four-electron sample of $\mathcal{L}_{\text {eff. }}^{e^{+}} e^{-}=\varepsilon_{e} \mathcal{L}=70.5 \pm 1.6 \mathrm{pb}^{-1}$. Here, $\varepsilon_{\mathrm{e}}$ represents the part of the detection efficiency, such as gaps in $\phi$, which are not included in the Monte Carlo calculation.

The analysis of muon pairs does not require the 4-cm fiducial cut, so including the corresponding correction factor leads to $\varepsilon_{\mu}=\varepsilon_{\pi}=1.450 \cdot \varepsilon_{e}$ and an effective luminosity for the non-electron sample of

$$
\mathcal{L}_{\mathrm{eft}}^{\pi^{+} \pi^{-}}=\mathcal{L}_{\text {eff. }}^{\mu^{+} \mu^{-}}=\varepsilon_{\mu} \mathcal{L}=102.3 \pm 2.3 \mathrm{pb}^{-1}
$$

The quoted error if the sum, in quadrature, of the errors listed in Table 5.2.

Another conceivable source of error is from radiative corrections to the leading-order QED graphs. One may suspect that the corrections could be different for the $e^{+} e^{-} \mu^{+} \mu^{-}$final state compared with the $e^{+} e^{-} e^{+} e^{-}$final state. It has been shown that the only significant contribution for either final state in leading order is from the two multiperipheral graphs, so we need only consider corrections to those two graphs. Therefore, the only difference in the calculations for the two final states comes from the muon-electron mass difference. The matrix elements are otherwise exactly the same. Now, the part that the mass plays in the result depends on kinematics; at high enough energy it should have very little effect. In fact, for the leading order cross sections, in the energy range and geometric
Table 5.2. Sourceg of error in the normalization derived from the electronpair sample.

\begin{tabular}{|c|c|}
\hline Source of Error & Percent Error \\
\hline trigger efficiency & \pm 0.8 (syst.) \\
\hline electron identification & \pm 0.5 (syst.) \\
\hline pre-tracking radiator & \pm 0.9 (stat.) \\
& \pm 1.5 (syst.) \\
\hline barrel shower counter & \pm 0.5 (etat.) \\
\hline acceptance correction & \pm 0.7 (stat.) \\
\hline size of $e^{+} e^{-}$sample & \pm 0.5 (stat.) \\
\hline Monte Carlo &
\end{tabular}

acceptance considered in this analysis, the difference of the two cross sections is oniy $3.7 \%$. Since the mass effect is so small for the leading order, and since the overall radiative corrections are known to be only of the order of $1 \%, t$ then it is reasonable to expect that the difference of the radiative effects for the two processes is much less that a percent and can be neglected.

Monte Carlo muon-pair events are analyzed just as electron pairs are, except that the effect of the Cerenkov identification is simply an overall loss of $1 \%$ of the events, and the shower counter latch efficiency is given by Fig. 5.5. The effective luminosity of Eqn. 5.1 yields 49,775 Monte Carlo muon pairs within the analysis cuts. Subtracting those from the data sample of muons and pions leaves a total of 20,984 pion pairs, with some remaining background, as seen in Fig. 5.7. The prominent peak around $1.2 \mathrm{GeV}$ is due to the $\int(1270)$, and one can sec that there also is a significant number of pion pairs produced in the continuum. In Chapter 8 this syectrum is fit to the theoretical model developed in Chapter 7 .

† Refer ta Section 4.10 for more discussion of the radiative corrections. 


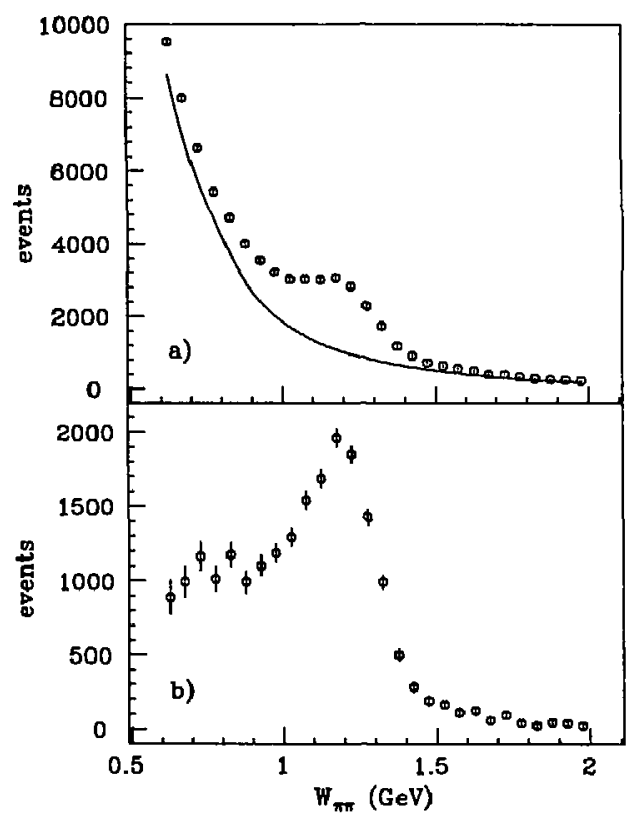

Figure 5.7. The pion-pair ard muon-pair invariant mass. (a) The data sample of pairs of non-electrons is represented by the points, while the smooth curve shows the predicted muon-pair spectrum. (b) The pion-pair spectrum which results after subtracting the muon-pair prediction.

\section{E.4 CONSIDERATION OF OTHER BACKGROUNDS TO $\gamma \gamma \rightarrow \pi^{+} \pi^{-}$}

Muon pairs are by far the largest background to the selection of pion pairs, but their are a few other minor sources of background which must be considered. First let us consider cosmic-ray muons. Most of them have been removed by cuts on the timing, but due to the resolution of the time-of-flight counters, there is a small tail toward short times which contributes some background. An upper limit on such background is estimated by roughly extrapolating the lower edge of the cosmic-ray peak below the cut at $8.0 \mathrm{ng}$. For that purpose, Fig. 5.3 is not useful because it showg the time distribution as it is before other cuts have been made, of which many preferentially reject cosmic rays. To study the background, the same histogram is reproduced, but the data are entered only after making all analysis cuts not related to the timing. The result shows that only $0.31 \%$ of the events have times above the $8.0 \mathrm{~ns}$ cut, and an upper limit on the cosmic-ray background not removed by the time cut is 15 events. That is an insignificant background, even when considering the distribution of $\widetilde{\beta}$, where all the cosmic rays fall in the one bin about $\tilde{\beta}=0$.

Next, we consider the background from beam-gas scattering. It should be characterized by a roughly uniform distribution of the z-position of the interaction. Figure 5.4 shows a histogram of the $z$-posilion of the estimated interaction point for events passing all analysis cuts except for the $z$ cut. Note the long, low tails outside the cut at $\pm 4.0 \mathrm{~cm}$. They are partly due to tails in the distribution of the colliding beams and tails in the tracking resolution, but they can be used to give an upper limit on the beam-gas contribution. It is reanonable to ansume no more than five events per bin coming from beam-gas scattering, which is a background of no more than $0.6 \%$ of the pion pairs.

The background from $e^{+} e^{-} \rightarrow$ hadrons is estimated by use of the Lund atringmodel Monte Carlo38 with radiative corrections included for the QED vertices.3 The cutoff energies for photons radiated from the incoming beams are set from $0.8561 E_{b}$ to $0.9801 E_{b}$, depending on the quark flavor produced, and the total cross section is $1.276 \cdot 3 \cdot \frac{11}{9} \cdot \sigma_{e}+e^{--\mu^{+}+\mu^{-}}=0.4817 \mathrm{nb}$. Of 46,378 events generated and simulated in the detector, only three pass all of the analysis cuts for $\gamma \gamma \rightarrow \pi^{+} \pi^{-}$. When normalized to the integrated luminosity of the data, that means that the background from this source is only about five events total, which is negligible.

For the same reason that Bhabha scattering does not contaminate the 
electron-pair sample, the process $e^{+} e^{-} \rightarrow \mu^{+} \mu^{-}$does not contaminate the pionpair sample. The contamination from tau-pairs is estimated in the same way as for the electron-pair analysis, and it is found to be at only the level of eight events. Again, such a level of contamination is small enorgh just to ignore.

Probably a larger source of contamination than $e^{+} e^{-} \rightarrow$ hadrons is inclusive hadron production, not including exclusive pion pairs, from $\gamma \gamma$ interactions. The $\gamma \gamma$ interactions produce events of low energy and multiplicity, so such events can more easily mimic pion pairs. Unfortunately, there is no simple model which can be used to estimate the background, and the cross section for $\eta y \rightarrow$ hadrons is not even known in detail empirically. However, one can estimate the background from the cumbination of all processes which produce at least four charged pions (plus beam-gas scattering) by observing the number of like-sign pion pairs in the data sample after all cuts other than the charge cut have been made. There are 70 such events, so one might expect approximately 70 more from the same source, but with a pair of oppositely charged pione detected. However, there are an estimated 116 beam-gas events in the data sample, which tend to have non-zero charge and might alone account for the 70 like-sign events. In fact, there are more events with charge +2 than -2 , as one would expect from beam-gas collisions. On the other hand, we have not yet accounted for background events with two charged pions and several neutral pions. There should be fewer of them than events with several charged pions, so it is reasonable to set an upper limit on the total background, including beam-gas events and $\gamma \gamma \rightarrow$ hadrons, of $150 \pm 50$ events, which is only $0.7 \%$ of the sample of pion pairs.

Not yet accounted for are two specific backgrounds, both of which can be accurately estimated and subtracted. The first is resonance production from two photons, where the resonance decays into two pions plus one-or-more photons. The only such resonance which can in that way contaminate the pion-pair data is the $\eta^{\prime}$. In particular, the decay mode $\eta^{\prime} \rightarrow p^{0} \gamma$ often produces two pions which pass all of the cuts, and the photon usually is of too low energy to be detected, or else it escapes through cracks in the detector.

The background from $\eta^{\prime}$ production is estimated by Monte Carlo, using as input the measurements of $\gamma \gamma \rightarrow \eta^{\prime}$ by the PLUTO collaboration. ${ }^{30}$ The $\eta^{\prime}$ state is generated as a Breit-Wigner resonance according to

$$
\sigma_{\tau \gamma \rightarrow \eta^{\prime}}=\frac{8 \pi W_{7 \gamma}}{m_{\eta^{\prime}}} \cdot \frac{\Gamma_{7 \eta^{\prime}} \Gamma_{\eta^{\prime}}}{\left(W_{7 \gamma}-m_{\eta^{\prime}}^{2}\right)^{2}-\Gamma_{\eta^{\prime}}^{2} m_{\eta^{\prime}}^{2}}
$$

with $m_{\eta^{\prime}}=0.257 \mathrm{GeV}, \Gamma_{\gamma \gamma}=3.80 \pm 0.26 \pm 0.43 \mathrm{keV}$, and $\Gamma_{\eta^{\prime}}=200 \pm 34 \mathrm{keV}$. When it is allowed to decay through any of its possible modes by using the decay programs of the Lund Monte Carlo,3 then one finds that only the $\rho^{0} y$ channel contributes any background.

The Lund programs do not take into account the large width of the $\rho^{0}$ or it: polarization, so it is necessary to study that channel in more detail. The $\rho^{0}$ has such a large width that a completely accurate description of the $\eta^{\prime}$ decay should properly include the three-body phase space. An adequate approximation for this purpose is instead to model the decay as a two-step process. After selecting the $\rho^{0}$ mass according to a Breit-Wigner distribution, the $\rho^{0}$ and $\gamma$ are produced in a uniform two-body phase space. The Breit-Wigner simply is cut off at the $\boldsymbol{\eta}^{\prime}$ mass, which is another simplifying assumption, but is not a large effeet. The $\rho^{0}$, which must, like the $\gamma$, have helicity \pm 1 , then is decayed into two pions with a center-of-mass angular distribution of $\sin ^{2} v$.

Of 6251 such events generated, corresponding to an integrated luminosity of $89 \mathrm{pb}^{-1}$, a total of 340 pass all of the analysis cuts for pion pairs, giving the mass spectrum shown in Fig. 5.8. The reason that the peak is not centered about the $\rho^{0}$ mass is because the efficiency for such events is rising sharply in that energy range. Including the uncertainties of the PLUTO measurement, of the measurement of the pion efficiency, and of the DELCO luminosity (122.5 $\left.\pm 4.5 \mathrm{pb}^{-1}\right)$, gives a total of $468 \pm 87$ events to be subtracted from the measured $\pi^{+} \pi^{-}$spectrum. 


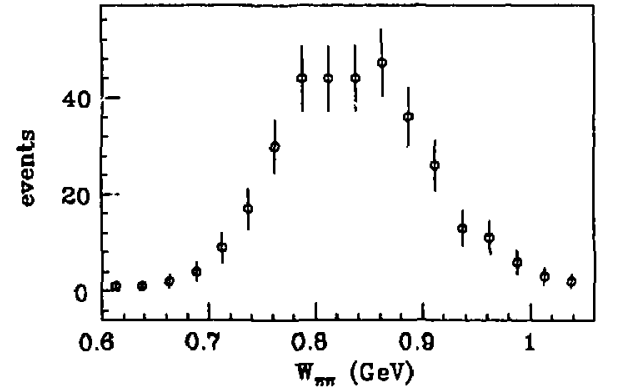

Figute 5.8. The $\eta^{\prime} \rightarrow \rho^{0} \gamma$ beckground $\pi^{+} \pi^{-}$mess spectrum corresponding to an integrated luminosity of $86 \mathrm{pb}^{-1}$.

The last background to consider is that due to the processes $\gamma \gamma \rightarrow K^{+} K^{-}$ and $\gamma \gamma \rightarrow p \bar{p}$. Such events often can be identified by time-of-flight measurements. However, only a fraction of the events in the data have usable timing information for each track. The approach used here is to consider only those events which do have good timing information available for both tracks and measure the fraction of kaon pairs plus proton pairs for each mass bin. $\dagger$ The number found of pairs of heavy particles is corrected for background and ineficiency induced by the timing cut3, and the result is scaled for each bin, by the ratio of the number of all pairs to the number with good timing, to give the background of the whole data set. Figure 5.9 shows the final result for the $K^{+} K^{-}, p \bar{p}$ background. Both it and the $\eta^{\prime}$ background are subtracted from the data in Chepter 8 when fitting the apectrum to theoretical models. However, these backgrounds are small enough that the change in the speetrum from what is seen in Fig. $5.7 b$ is hardly visible.

† Refer to Chapter 9 for a complete discumion of the time-ol-8ight analyais procedures.

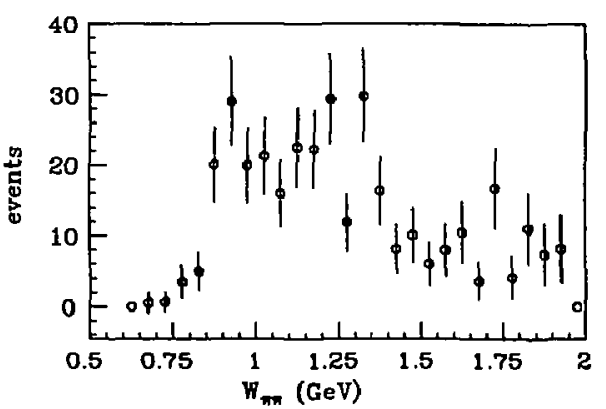

Figure 5.9. The $K^{+} K^{-}$plus $\mathrm{pp}$ bockground to the $\gamma \boldsymbol{\gamma} \rightarrow \pi^{+} \pi^{-}$ measurement, as measured from the data and corrected for the timeof-fight acceptance and efficiency.

\subsection{The Tagged Pion-Pair SPegtrum}

The tagged analysis proceeds much the same as the untagged, and the analyais of the luminosity counter tags is done exactly the same as for the four-electron channel. The cuts are the same as for the untagged analysis, except for the cut on the luminasity counter energy and the cuts on the total transverae momentum $k_{\perp}$ of the two tracks observed in the central detector. Because a strict cut on $k_{\perp}$ cannot be made, the rejection of background is a more serious problem than for the untagged analysis. Therefore, the cuts on noise in varjous countera and in the outer drift chambers become more important. They reduce the final sampie of non-electron pairs by $5.5 \%$. Using the fact that $2.0 \%$ of the final gample of electron pairs is eliminated by the same cuts and assuming the same effect on muon pairs, we estimate that the cuts reduce the sample of pion pairs by about $15 \%$.

An attempt is made to use the measurement of $k_{\perp}$ to reduce the background a little further. Assuming that only one of the beam electrons scatters at a lurge angle, then the trangverse momentum of the tagged electron should balance that of the two particles observed in the central detector. Unfortunately, the luminowity 


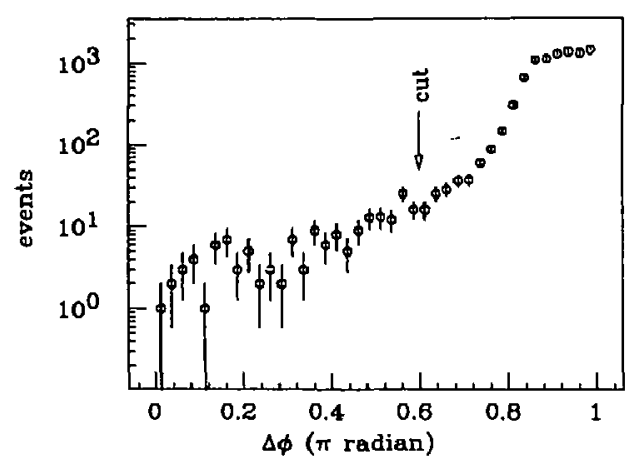

Figure 5.10. Transverse momentum cut for tagged data. $\Delta \phi$ is the angle in the transverse plane between the direction of the sum of the momenta of the two electron tracks and the direction of the center of the hit luminosity counter.

counters do not give a good measurement of the transverse momentum. They tell only whether it is larger than about $0.3 \mathrm{GeV}$ and what the azimuthal angle is within \pm 60 degrees. Therefore, the only cuts made are to require that the measured $k_{\perp}$ be larger than $0.1 \mathrm{GeV}$ and that the angle $\Delta \phi$, between the vector $\vec{p}_{t}^{1}+\vec{p}_{t}^{2}$ measured in the central detector and the $\phi$ direction of the center of the hit luminosity counter, be larger than $0.6 \pi$ radians. Figure 5.10 shows a histogram of $\Delta \phi$ for tagged electron pairs, made before any of the noise cuts. Only $1.8 \%$ of those events are eliminated by the cut, while it eliminates $3.7 \%$ of the tagged non-electron pairs. Thus it is evident that even such a loose cut helps substantially to reduce the background.

Because of the requirement of a high energy tag in the luminosity counters, backgrounds from all sources other than two-photon scattering are greatly reduced. For example, when the time difference from one side of the detector to the other is plotted before other cuts are made, only 5 of the 989 events with an angle between tracks greater than 168.5 degrees have $\Delta t>8.0$ ns. Thus the cosmic ray background is negligible, and likewise the beam-gas and $e^{+} e^{-} \rightarrow$ hadrons backgrounds are negligible. The remaining background, except for that from $\eta^{\prime}$ production and charged kaon and proton pairs, is estimated from the number of events found with non-zero charge. There are 227 such events after all other cuts have been made, compared with only four electron pairs of non-zero charge. There should be an equal number of zero-charged events from the same background source, and there could be as much as about half that many again of events from the same background but with extra neutral, rather than charged, pions. The charged background is relatively certain, while we can only guess at the number of neutral pions which may have escaped detection. Hence the background from multi-hadron production is estimated to be $560 \pm 112$ events.

The backgrounds from $\eta^{\prime}$ production and from charged kaon and proton pairs are determined in the same way as for the untagged analysis. In this case, the number of proton and kaon pairs (45 total) is completely insignificant compared with the statistical precision of the pion-pair sample, amounting to no more than four events in any single bin. The calculation of $\eta^{\prime}$ production assumes a $\rho^{0}$-pole form factor, $1 /\left(1-q^{2} / m_{\rho}^{2}\right)$, which has been found by the PLUTO collaboration to give an adequate description of their data. ${ }^{3 \theta}$ The number of $\eta^{\prime}$ yielding pion pairs to be subtracted from data is only $72 \pm 16$.

In order to obtain the normalization for subtracting muon pairs, the tagged electron pairs are analyzed within the trigger acceptance defined by the fiducial cut $4 \mathrm{~cm}$ from the shower counter edges. The number found in the range $0.7<W_{\pi \pi}<2.0 \mathrm{GeV}$ is 3719 events. Again, a correction must be made for bremsstrahlung energy loss in the beampipe and drift chamber material. Using again the full simulation of the detector, including the EGS Monte Carlo, the loss of electron pairs relative to muon pairs is $0.931 \pm 0.016$ for the $1982 / 1989$ data, $0.966 \pm 0.017$ for the 1984 data, and $0.943 \pm 0.016$ overall. The effect is less for the tagged analysis than for the untagged partly because for the former there is no cut 
on $k_{\perp}$. In general the two differ kineinatically, so some difference must be expected in any case. Comparing the data with a calculation from the Vermaseren Monte Carlo and applying the correction gives an effective luminosity of $62.7 \pm 1.6 \mathrm{pb}^{-1}$.

For tagged non-electron pairs, the aS trigger is redundant to the LS trigger, which requires only one shower counter in coincidence with the luminosity counter. Therefore, there are some events in which only one pion or muon has hit and fired a shower counter. But both particles still are required to fall within the shower counter acceptance. In cases where one of the particles does not latch a shower counter, the tracking information is used for that particle to determine whether it is within the acceptance. When the acceptance is so defined, there is an increase of the number of non-electron pairs by a factor of $1.501 \pm 0.024$ over the number found within an arceptance defined by the 4-cm fiducial cut. Including that correction results in an effective luminosity fot the tagged non-electron sample of

$$
\mathcal{L}_{\text {eff. }}^{t s 8}=94.1 \pm 3.3 \mathrm{pb}^{-2}
$$

where the quoted error is the sum, in quadrature, of the errors listed in Table 5.3.

Table 5.3. Sources of error in the normalization derived from the tagged electron-pair sample.

\begin{tabular}{|c|c|}
\hline Source of Errot & Fercent Error \\
\hline trigger efficiency & \pm 0.8 (syst.) \\
\hline electron identification & \pm 0.5 (syst.) \\
\hline pre-tracking radiator & $\begin{array}{l} \pm 1.7 \text { (stat.) } \\
\pm 1.5 \text { (syst.) }\end{array}$ \\
\hline barrel shnwer counter & \\
\hline acceptance correction & \pm 1.6 (stat.) \\
\hline size of $e^{+} e^{-}$sample & \pm 1.6 (stat.) \\
\hline Monte Cario & \pm 0.9 (stat.) \\
\hline
\end{tabular}

From the data, a total of 9366 tagged non-electron pairs pass all of the cuts. The invariant-mass spectrum for those events, calculated with an assumption of pion masses, is displayed in Fig. 5.11a. Using the luminosity of Eqn. 5.3, the muon-pair spectrum is produced as for the untagged analysis, except that in this case it is essential to use the Vermaseren Monte Carlo generator. The calculation yields 6654 muon pairs, and subtracting those leaves 2712 pion pairs. Subtracting the estimates of the other backgrounds leaves the 2034 events for which the $\pi^{+} \pi^{-}$ invariant-mass spectrum is displayed in Fig. 5.11b.

As in the $\pi^{+} \pi^{-}$mass spectrum from untagged events, a peak near the $f$ is prominent, and there also is a large amount of continuum production. As far as data analysis is concerned, what remains to be done is to measure loo the angular distribution for pions pairs and to correct the observed distributions for resolution and efficiency effects. Then, to finish the measurement, a model must be constructed which includes both continuum and resonance production, and it must be fit to the data. But first, let us finish the analysis necessary for determining the detection efficiency for pion pairs. 


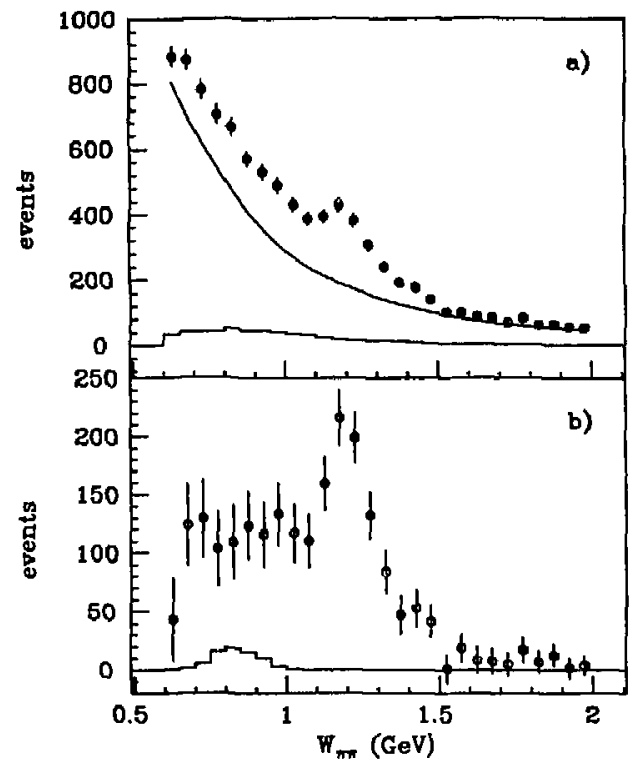

Figure 5.11. The invariant mass spectrum for tagged non-electron pairs. (a) The points represent the measured data, the smooth curve is the prediction for the $\mu^{+} \mu^{-}$background, and the histogram drawn as a line is the estimated of the random background, obtained from the spectrum of pairs with nonzero charge. (b) The points represent the $\pi^{+} \pi^{-}$spectrum obtained by subtracting all background. The histogram around the $\rho^{0}$ mass is the calculated background $\mathrm{fr}^{\prime} \mathrm{m} \boldsymbol{\eta}^{\prime}$ production.

\section{Measurement of the $\pi^{+} \pi^{-}$Trigger Efficiency}

$A$ good underatanding of the trigger efficiency for electrons and muons has allowed an accurate subtraction of the background from the sample of non-electron pairs. But before any information can be extracted from the resulting $\pi^{+} \pi^{-}$ spectrum, it must be corrected for the trigger inefficiency of pion pairs. The necessary correction differs from that calculated for muon pairs because of nuclear interactions of the pions with material between the beam-line and the scintillators of the shower counters. In order to latch a shower counter, an incident pion, or its decay or reaction products, must penetrate, when at normal incidence, roughly $6 \mathrm{~cm}$ of aluminum, which is 0.15 nuclear interaction lengths, followed by $2.5 \mathrm{~cm}$ of lead, which also is 0.15 nuclear interaction lengths. It is apparent that a significant fraction of the pions must undergo some sort of inelastic nuclear interaction, but it is impossible to make a simple estimate of the effect on the trigger offieiency. The best approach is to try to measure it from dats, as has been done for the electron pairs.

\subsection{ShOWER COUNTER LATCh EFFIGIENCY FROM TAGgED DATA}

It is unfortunate that there is no trigger redundancy to exploit in the sample of untagged non-electron pairs, but it is possible to use tagged dats for measuring the shower counter latch efficiency, albeit with less statistical significance. The luminosity counter tag in coincidence with a single shower counter latch provides an LS trigger, which is partially redundant to the $2 S$ trigger. Since in all cases at least one shower counter is required to latch, then it is necessary, as in the analysis of the electron pairs, to unfold the true efficieney from the trigger-biased efficiency plotted from data. However, there is an additional problem in this case which even further reduces the statistical significance of the measurement. About $3 / 4$ of the non-electron sample consists of muon pairs, so their contribution must be accounted for when unfolding the pion shower counter latch efficiency from the data. 
In this chapter we are concerned only with the inefficiency caused by pions stopping before reaching the second layer of scintillator-inefficiency due to gaps between counters already has been accounted for. It is useful to review why that is so. Recall that for non-electron pairs, no cut is made on the extrapolation of the drift chamber tracking information out to the shower counter region. To do so would cause errors due to limitations of the survey accuracy and tracking resolution and to multiple scattering in material preceding the shower counters. Survey and tracking errors cancel when normalizing pions and muons to the electron measurement, but scattering, which may include nuclear interactions, and electromagnetic showers depend on the particle type.

The problem of electromagnetic showers is resolved by analyzing the electron pairs, for the purpose of normalization, within a fiducial cut placed $4 \mathrm{~cm}$ from the edges of the shower counter modules. For that it still is necessary to use the tracking information, but the cut is far enough from the edge that the errors have no effect. For non-electrons, no cut at all is made on the tracking information; the acceptance simply is defined by the trigger, which requires both particles actually to latch a shower counter. Thus the geometric acceptance is guaranteed to be the same for pions as for muons. Even though pions may scatter more than muons, because of nuclear interactions, just as many must scatter into the acceptance as scalter out, so that is not a problem. There may be a slight difference coming from inelastic nuclear interactions of pions in the aluminum adjacent to the shower counters, but it must be a much smaller effect than for electromagnetic showers. That is because whereas every electron hitting the aluminum produces a shower, only a small fraction of the incident pions produce a nuclear or electromagnetic avalanche. Such effects change the acceptance for electrons by only a couple of percent, so they are believed to be negligible for pions.

Therefore, the effect of nuclear interactions on the pion efficiency can be analyzed completely separate from edge effects. To do so requires making a fiducial cut inside the shower counters, and as one can see from Fig. 6.1, a 4-cm cut is

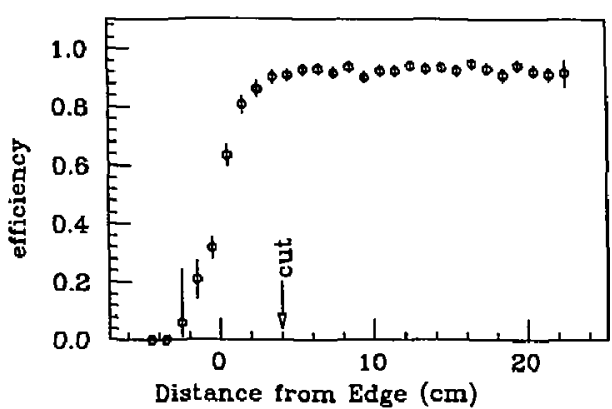

Figure 6.1. Resolution of the shower counter edge for non-electron tracks. The shower counter latch efficiency for each particle from tagged nonclectron pairs is plotted as a function of the measured distance from the nearest edge of the counter.

adequatc. Except for the region within $4 \mathrm{~cm}$ of the couster edge, the efficiency is flat, and the only explanation for the cause of the observed inefficiency is that a fraction of the rions in the sample are stopped before traveling far enough to latch a counter.

To produce efficiency plots from data, tagged events are analyzed just as in Chapter 4, except that some cuts are made on the time-of-flight information in order to reject kaon and proton pairs. Since kaons and protons range-out with lower initial momentum than do pions and muons, their presence could confuse the efficiency measurement. Both tracks in each event are required to be within the acceptance of the time-of-fight system, as determined from tracking information. It is not possible to cut on the timing information of all tracks, however, because to do so would require all particles to produce a significant signal in the timeof-flight counters. Since almost half of the material of concern here, in units of nuclear interaction lengths, is in front of the time-of-light system, that would cause a large bias in the result. However, it is possible to reject those events with 
particles which do have good times and are consistent with being kaons or protons, and doing so removes most of the problem. Of the non-electron tracks with good timing information, $2.1 \%$ are kaon or proton candidates, compared with $0.06 \%$ for the electron sample. The remaining number of kaons and protons is too negligible to have any noticeable effect on the efficiency plot.

From the resulting sample of non-electron pairs, a histogram of the shower counter latch efficiency is accurnulated using all those tracks which satisfy the $4-\mathrm{cm}$ fiducial cut, resulting in the 12,175 entries shown in Fig. 6.2. Using the electron pairs for normalization, the fraction of muon pairs in the sample is determined to be $0.72 \pm 0.02 \%$. Their contribution to the efficiency plot is calculated by using the Vermaseren Monte Carlo and the efficiency parameterization given in Fig. 5.5. Recall that for muons the efficiency is $100 \%$ for momenta well above $0.18 \mathrm{GeV}$, below which they range out before reaching the second layer of scintillator (see Fig. 5.5). Well above that threshold, the only conceivable source of inefficiency is from light collection and the electronics. That possibility is removed by analyzing events from the reaction $e^{+} e^{-} \rightarrow \mu^{+} \mu^{-}$, for which the muons always are above Cerenkov threshold and therefore have redundant triggers. Of 3379 such muons which eatisfy the $4-\mathrm{cm}$ fiducial cut on the shower counters, only three fail to latch a shower counter. Thus the counters are essentially $100 \%$ efficient for aingle minimum-ionizing particles which actually pass through the scintillators.

In order to model the contribution of pions, the form used to parameterize their shower counter latch efficiency is

$$
E(p)=0.995 \cdot\left[1-0.6 \cdot \exp \left(-\frac{p-0.177}{0.0219}\right)\right] \times\left[1-0.6 \cdot \exp \left(-\frac{p-p_{0}}{\tau}\right)\right]
$$

The first exponential factor serves to produce the sharp cutoff due to electromagnetic range-out, and the second is adjusted to reproduce the efficiency observed in the data. For the same reasons as in the measurement of the electron efficiency, it is only necessary to parameterize the momentum dependence. However, one must

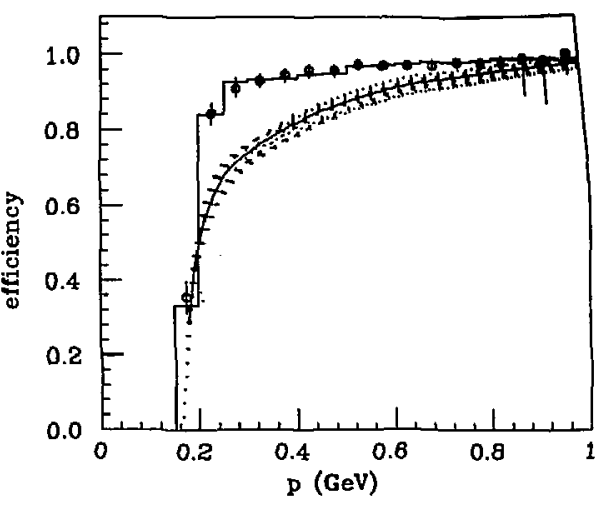

Figure 6.2. The shower counter latch efficiency for non-electrons as a function of the particle momentum. The points with error bars are measured from the tagged non-electron pairs, and the overplotted histogram is the best fit from a Monte Carlo calculation. The solid smooth curve is the parameterization used in the Monte Carlo for the pion shower counter latch efficiency, and the dotted curves show the range of uncertainty in the fit.

be sure that the Monte Carlo reproduces reasonably well the actual momentum distributions for the pions, so that the trigger bias is accurately reproduced. That is accomplished by using the $\gamma \gamma$ luminosity function appropriate for single tagged events in conjunction with the model for $\gamma \gamma \rightarrow \pi^{+} \pi^{-}$, which is introduced in Chapter 7.

The fitting procedure begins with generation of an efficiency histogram with 35,000 entries from Monte Carlo muon pairs. Then the pion-pair Monte Carlo is run, and for each of 100 pairs of trial values for po and $\tau$ of Eqn. 6.1, an efficiency histogram is produced by adding 13,611 pion entries to the existing muon histogram. Comparing the 100 Monte Carlo histograms with the one from data produces a $10 \times 10$ matrix of $x^{2}$ values. Those entries which are no more than 
one unit of $\chi^{2}$ above the minimum roughly form an elongated ellipse, showing an expected large negative correlation between $p_{0}$ and $\tau$. The center of the ellipse $\left(p_{0}=0.070, \tau=0.270\right)$ is taken as the best fit and the resulting efficiency curve is shown as the solid smooth curve of Fig. 6.2. The set of dotted curves represent the pairs of values forming the perimeter of the ellipse and therefore indicate the one-standard-deviation uncertainty limits of the efficiency measurement.

This result includes, in addition to losses from : uclear and electromagnetic interactions, some losses resulting from those weak decays of pions which occur after leaving the inner tracking chambers. However, one expects the effects of such decays to be small. In fact, when pion decays are added to the Monte Carlo aimulation, which does not include nuclear interactions, the detection efficiency decreases overall by less than $1.5 \%$. With nuclear interactions included, the effect could be of the opposite sign. Because of the small difference in mass between the pion and muon, such decays usually result in a muon going in about the same direction as the initial pion. Therefore, it is conceivable that decays could actually increase the trigger efficiency, since the latch efficiency for muons is very high. Whatever the case may be, the effect is almost negligible and most of it already is included in the efficiency measurement. The remaining effect from decays which occur within the tracking volume and result in a loss of the track itself is negligible.

\subsection{Calgulation of the Shower Counter Latch Efficiency}

It is worthwhile to attempt a direct calculation of the pion shower counter latch efficiency just to check that what has been measured makes physical sense. However, the result of the calculation cannot be expected to agree with the data to the extent that the EGS calculation reproduces the electron shower counter latch efficiency. In the electromagnetic case the details of the physical processes are well known, but for nuclear interactions one must rely on relatively crude models which try to reproduce features of physical processes which are not so well understood and which are great in variety. Furthermore, a determination of the

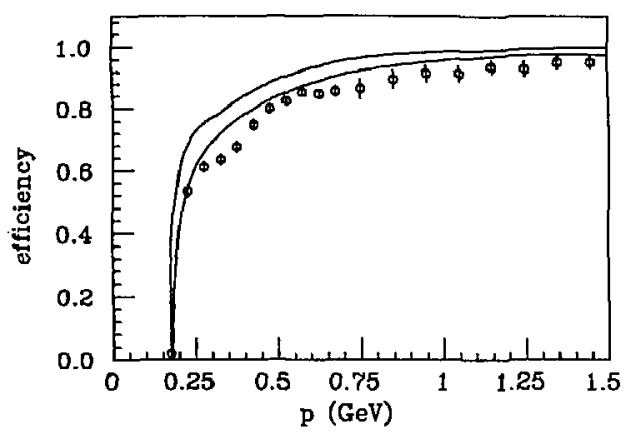

Figure 6.3. HETC calculation of the shower counter latch efficiency for pions. The points show the results of two HETC calculations covering two momentum ranges, and the smooth curves show the range of uncertainty in the measurement from data, including both the statistical uncertainty and the uneertainty in the rormalization of the muon contribution.

latch efficiency requires a very detailed calculation, because it takes only one of many possible charged particles produced fram a reaction to latch a counter.

HETC $^{30}$ is used along with the $\mathrm{EGS}^{29}$ code to simulate nuclear and hadronic interactions in the detzctor material. EGS is called only when a $\pi^{0}$ is produced from a nuclear interaction. HETC uses what is called the Intermediate Energy Intranuclear-Cascade-Evaporation Model for pion-nucleus inelastic collisions (except for $\pi p$ reactions, which are not relevant here). Running the Monte Carlo model for $e^{+} e^{-} \rightarrow e^{+} e^{-} \pi^{+} \pi^{-}$with the HETC-EGS simulation results in the histogram of Fig. 6.3. The efficiency from the calculation is consistently a little low, but it does show the same trend as what is observed in the data.

Since the HETC calculation does reproduce the general features of the inefficiency seen in the data, it is interesting to look a little more decply into 
the calculation to see what types of physical processes are responsible for the loss of efficiency. One finds that a few of the pions which fail to latch a shower counter have undergone a charge exchange reaction such as $\pi^{-} p \rightarrow \pi^{0} n$, and the resulting electromagnetic shower has not penetrated far enough. Then there are a few other low-multiplicity reactions for which a neutron has carried off most of the momentum. However, most of the cases of latch failure are due to reactions in which several protons and neutrons more-or-less share equally the momentum in the final state. The neutrons seldom produce any interactions in the scintillators, and the protons typically do not have enough momentum to penetrate through much lead. In general, the reactions responsible for the loss of efficiency are, at least in the Monte Carlo, quite complicated, and the result of whether the reaction products can latch the counter depends critically on how the momenturn is distributed among the protons and pions of the fintal state. Therefore, it is enough to conclude that the calculation does verify the momentum dependence, and approximately the magnitude, of the incficiency seen in the data-the measurement is what is actually used in the following analysis.

\subsection{Trigger Efficiency for Pion Pairs}

When fitting the invariant mass spectrum of the pion pairs to a model, it is necessary to know the uncertainty in the trigger efficjency as a function of the pion-pair isvariant mass. That is obtained by using the Monte Carlo to transform the upper and lower limits on the shower counter latch efficiency, shown in Fig. 6.2 as functions of momentum, into upper and lower limits on the trigger efficiency. It also is necessary to include the uncertainty from the normalization of the muon Monte Car!o, as obtained from the electron-pair measurement. If $\varepsilon$ is the efficiency measured for the sum of pions and muons, and $f$ is the fraction of muons, then the efficiency of the pions alone is approximately

$$
\varepsilon_{\pi}=\frac{\varepsilon-f}{1-f} .
$$

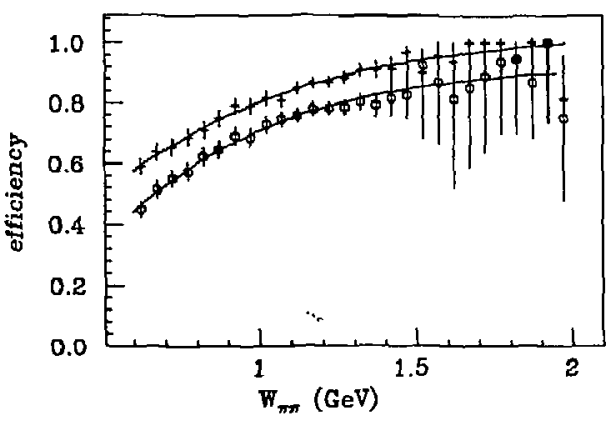

Figure 6.4. The pion-pair trigger efficiency as a function of invariant mass. The upper and lower sets of points, through which smooth curves are drawn, are generated by Monte Carlo, using as input the measured upper and low limits of the shower counter latch efficiency.

Using the predicted fraction $f$ for each momentum bin along with the eatimated $2.9 \%$ uncertainty in $f$, this equation is used to find the error in $\varepsilon_{\pi}$ for each bin. That error actually already is included in the limits shown by the contours in Fig. 6.3, and using those contours in the Monte Carlo produces the result shown in Fig. 6.4. The percent difference between the two curves gives the uncertainty in the efficiency measurement for each invariant-mass bin. It can be adequately paraneterized as a parabola with zero slope at the upper end of the mass range:

$$
\varepsilon(W)=\varepsilon_{1}^{\pi}\left[\frac{a-\frac{a^{2}}{2 b}-W+\frac{1}{2 b} W^{2}}{a-\frac{a^{2}}{2 b}-\frac{1}{2} b}\right]+\varepsilon_{2}^{\pi}\left[\frac{-\frac{1}{2 b}+W-\frac{1}{2 b} W^{2}}{a-\frac{a^{2}}{2 b}-\frac{1}{2} b}\right],
$$

where $a=W_{1}=0.6 \mathrm{GeV}, b=W_{2}=2.0 \mathrm{GeV}, \varepsilon_{1}^{\pi}:=0.10$, and $\varepsilon_{2}^{\pi}=0.05$, When fitting the model for $\gamma \gamma \rightarrow \pi^{+} \pi^{-}$to the $\pi^{+} \pi^{-}$invariant-mass spectrum (Chapter 8), Eqn. 6.1 with $p_{0}=0.070$ and $\tau=0.270$ is used in the Monte Carlo for the pion shower counter latch efficiency, while Eqn. 6.3 gives the uncertainty in the pion-pair detection efficiency. 


\section{Theoretical Models for $\gamma \gamma \rightarrow \pi^{+} \pi^{-}$}

In principle, the process $\gamma \gamma \rightarrow \pi^{+} \pi^{-}$should be described by QED coupling of the photons to quarks, with the interactions of the quarks and gluons within the mesons calculated from quantum chromodynamics (QCD). In practice, however, such calculations may only be carried out in the perturbative regime of QCD. If the pion-pair invariant mass is large enough and the pions are produced at large angles with respect to the incoming photons, then the amplitude factors into two parts: $4^{0}$ The first consists of a parton distribution amplitude $\phi\left(x_{i}, Q\right)$ for each pion-the probability ampli ude for finding valence quarks in the pion, each carrying a fraction $x_{i}$ of the picin's momentum, integrated over transverse momenta up to $k_{\perp i} \approx Q$, where $Q$ is the momentum transferred in the process. The second is a hard-scattering amplitude $T$ for scattering the clusters of collinear vaience partons from each pion. Thus the amplitude is

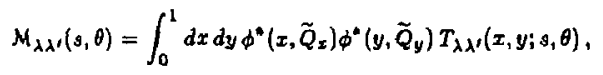

where $\tilde{Q}_{x} \approx \min (x, 1-x) \sqrt{s}|\sin \theta|$ and $\lambda \lambda^{\prime}$ arc the photon helicities.

Brodsky and Lepage ${ }^{1}$ have carried out this calculation with leading-order QCD. The parton distribution functions are determined by relating them to the known pion form factor $F_{\pi}(s)$, and the hard scattering amplitudes are calculated from the diagrams shown in Fig. 7.1. There remains some unknown $x$ dependence of $\phi(x, Q)$ for which issumptions must be made. However, the result is almost completely independent of those assumptions and takes the form

$$
\frac{\mathrm{d} \sigma}{\mathrm{d} t}=\frac{4\left|F_{n}(s)\right|^{2}}{1-\cos ^{4} \theta} \cdot \frac{\mathrm{d} \sigma}{\mathrm{d} t}\left(\gamma \gamma \rightarrow \mu^{+} \mu^{-}\right),
$$

where $F_{\pi}(s) \approx 0.4 \mathrm{GeV}^{2} / \mathrm{s}$, and $s$ and $t$ are the usual Mandelstam invariants.

\footnotetext{
+ For the reaction $a+b \rightarrow c+d$, one defines the Mandelatam invariante as $s=\left(p_{a}+p_{b}\right)^{2}$ $t=\left(p_{c}-p_{a}\right)^{2}$, and $u=\left(p_{1}-p_{a}\right)^{2}$. The thrce are related by the simple expression $i+t+u=$ $m_{a}^{2}+m_{b}^{2}+m_{c}^{2}+m_{d}^{2}$.
}
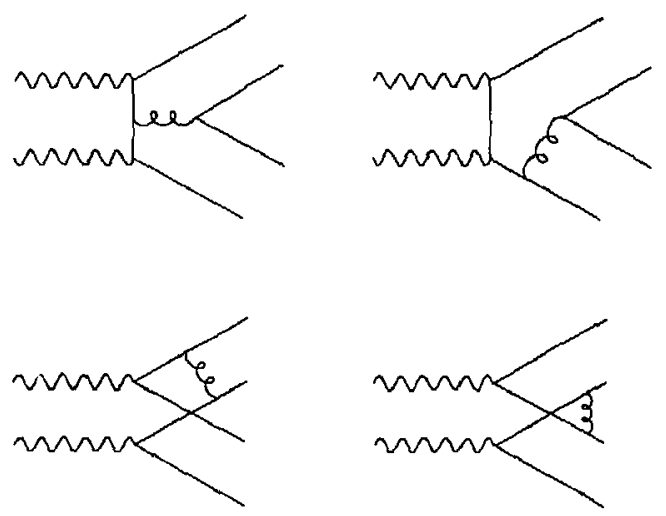

Figure 7.1. Leading-order QCD diagrams contributing to the procest $\gamma \gamma \rightarrow \pi^{+} \pi^{-}$. The amplitudes are calculated with the quarks collinear with the incoming and outgoing pions

Equation 7.2 is a beautiful and simple parameter-free prediction, but it is valid only when s-channel resonance effects car be neglected. We have seen that the energy range accessible to DELCO is in fact dominated by resonance effects. Furthermore, that energy range probably is too low to give momentum transfers large enough to place the process in a regime where QCD perturbation theory becomes valid. Unfortunately, it is not possible to extend the DELCO measurement to higher energies, because there is no way to separate pions from muons. As seen in Eqn. 7.2, the pion-pair cross section at high energies falls like $1 / s^{2}$ relative to the muon-pair cross section. Therefore, the background quickly dominates the signal to such an extent that there is no possibility of subtracting it.

It is clear that a more phenomenological model nust be used to otudy the low energy pion-pair spectrum. The physical picture of single-hadron exchange 
and formation has been useful in some low-energy phenomenology and may be expected to work well for this case. One expects a large contribution from singlepion exchange in the $t$-channel, $\gamma \pi^{ \pm} \rightarrow \gamma \pi^{ \pm}$, which may be used to describe the low-energy continuum production, and the s-channel resonance effects can be modeled according to a relativistic Breit-Wigner shape. Such a model has been used by a number of experinents, ${ }^{41}$ and it has been found to give a good description of the data when interference between the single-pion exchange and the resonance production is included. We will see that such a model also gives a good description of the DELCO data. But first it is important to consider the motivations for the model, its theoretical problems and limitations, and also some possible extensions.

\subsection{DESCRIPTION OF THE INCOMING TWO-PHOTON STATE}

It is necessary tirst to describe the initial two-photon state in such a way that the conservation laws for angular momentum and parity are easily incorporated into the matrix elements. The formulas used for partial wave expansions can be found in the textbook of $\therefore$. Werle, ${ }^{42}$ and the phase and normalization conventions used here are the same as his. Let $R(\alpha, \beta, \gamma)$ represent the operator to produce a rotation through the three Euler angles about, respectively, the $z$ axis, the $y$ axis, and the new $z$ axis, and let $L_{z}(v)$ represent a Lorentz boost in the $\hat{z}$ direction to a relative velocity $v$, with no rotation of the coordinate axes. Then the basis states for a single particle are written as

$$
|m s ; \tilde{p} \lambda\rangle=|m s ; \phi \theta p \lambda\rangle=R(\phi, \theta, 0) L_{z}(v)|m s ; 000 \lambda\rangle .
$$

This notation represents a particle of mass $m$, spin $s$, and helicity $\lambda$, moving as a plane wave with momentum $p$ in the direction given by the polar angles $\phi$ and $\theta$ (from here on, the notation ms will be suppressed). The state $|000 \lambda\rangle$ is just the particle $-t$ rest, with its spin component in the $\hat{z}$ direction given by $s_{x}=\lambda$. The important phase convention assumed is ${ }^{43}$

$$
|0 \pi p \lambda\rangle=|00-p \lambda\rangle=e^{-i \pi \delta} R(\pi, \pi, 0) L_{\mathbf{x}}(v)|000 \lambda\rangle,
$$

and the normalization is $\left\langle\vec{p}^{\prime} \lambda^{\prime} \mid \vec{p} \lambda\right\rangle=E \delta^{3}\left(\vec{p}^{\prime}-\vec{p}\right) \delta_{\lambda^{\prime} \lambda}$, where $E=\sqrt{m^{2}+\vec{p}^{2}}$.

A state with two particles may be formed as a direct product of the two singleparticle states. In the center-of-mass system, for particle 1 going in the direction $(\phi, \theta)$, the appropriate definition is

$$
\left.\left|\vec{P}=0 ; \phi \theta p \lambda_{1} \lambda_{2}\right\rangle=\sqrt{\frac{p}{W}} \cdot R(\phi, \theta, 0) \mid 00 p \lambda_{1}\right)\left|00-p \lambda_{2}\right\rangle,
$$

where $W^{2} \equiv P^{\mu} P_{\mu}$ and $P=p_{1}+p_{2}$. The normalization follows from that of Eqn. 7.3 and is found to be

$$
\left\langle\vec{P}^{\prime} ; \Omega^{\prime} \lambda_{1}^{\prime} \lambda_{2}^{\prime} \mid \vec{P}_{i} \Omega \lambda_{1} \lambda_{2}\right\rangle=\delta_{\lambda_{1}^{\prime} \lambda_{1}} \delta_{\lambda_{2}^{\prime} \lambda_{2}} \delta^{4}\left(P^{\prime}-P\right) \delta^{2}\left(\Omega^{\prime}-\Omega\right),
$$

where we have made use of the identity

$$
\frac{\mathrm{d}^{3} p_{1}}{E_{1}} \frac{\mathrm{d}^{3} p_{2}}{E_{2}}=\frac{p}{W} \mathrm{~d}^{4} P \mathrm{~d} \phi \mathrm{d} \cos \theta=\frac{p}{W} \mathrm{~d}^{4} P \mathrm{~d}^{2} \Omega,
$$

One should note that the basis vectors of Eqn. 7.3 are not defined properly for the case of real photons, which have mass zero and, hence, no rest system. They may still be defined the same way, however, if one assumes as a starting point the vector $\left|00 p_{0}\right| \lambda|\rangle$ rather than $|000 \lambda\rangle$ and keeps in mind that in this case $\lambda$ is a property of the particle, with a value of $+s$ or $-s$, and does not change from one Lorentz frame to another. Then the two-photon state may be defined exactly as in Eqn. 7.5. Note, however, that whereas Eqn. 7.5 may usually be expanded in another basis represented by $l$, the relative angular momentum, and $\sigma$, the total spin, that cannot be done for the case of two massless particles. 
Another technical point is that the two photons, being identical bosons, must be in a state which is symmetric under interchange of the two. Interchanging the two particles in Eqn. 7.5 is equivalent to interchanging the helicities and then rotating about the $y$ axis by $\pi$ radians. Therefore the four possible incoming states for the two photons are, in their center-of-mass system traveling in the $z$ direction with momenta $\pm g$,

$$
\begin{aligned}
\psi_{J_{x}=0}^{1} & =\sqrt{\frac{1}{2}}[1+R(0, \pi, 0)|| \vec{P}=0 ; 00 q++\rangle \\
\psi_{J_{x}=0}^{2} & =\sqrt{\frac{1}{2}}[1+R(0, \pi, 0)\}|\vec{P}=0 ; 00 q--\rangle \\
\psi_{J_{x}=2}^{3} & =\sqrt{\frac{1}{2}}[|\vec{P}=0 ; 00 q+-\rangle+R(0, \pi, 0)|\vec{P}=0 ; 00 q-+\rangle] \\
\psi_{J_{x}=-2}^{4} & =\sqrt{\frac{1}{2}}[|\vec{P}=0 ; 00 q-+\rangle+R(0, \pi, 0)|\vec{P}=0 ; 00 q+-\rangle] .
\end{aligned}
$$

Our goal is to project out components of the incoming $\gamma \gamma$ states which have definite spin and parity. A state of definite angular momentum may be formed from the basis defined in Eqn. 7.5 according to 44

$$
\left|\vec{P}=0 ; M[W J] \lambda_{1} \lambda_{2}\right\rangle=\sqrt{\frac{2 J+1}{\pi}} \int d \Omega \bar{D}_{M \lambda}^{J}(\phi, \theta, 0)\left|\vec{P}=0_{;} \phi \theta W \lambda_{1} \lambda_{2}\right\rangle,
$$

where $\lambda \equiv \lambda_{1}-\lambda_{2}$ and

$$
D_{m^{\prime} m}^{j}(\alpha, \beta, \gamma)=e^{i m^{\prime} \alpha} d_{m^{\prime} m}^{j}(\beta) e^{i m \gamma} \equiv\left\langle j m^{\prime}|R(\alpha, \beta, \gamma)| j m\right\rangle
$$

If $\mathbf{P}$ is the operator for space inversion and $A_{1,2}$ is the exchange operator, then ${ }^{45}$

$$
\begin{aligned}
\mathbf{P} \mid \vec{P} & =0 ; M\left[W J\left|\lambda_{1} \lambda_{2}\right\rangle=\eta_{1} \eta_{2}(-1)^{J-s_{1}-s_{2}}\left|\vec{P}=0 ; M[W J]-\lambda_{1}-\lambda_{2}\right\rangle\right. \\
A_{1,2} \mid \vec{P} & \left.=0 ; M[W J] \lambda_{1} \lambda_{2}\right\rangle=(-1)^{J-2 s} \mid \vec{P}=0 ; M\left[W J\left|\lambda_{2} \lambda_{1}\right\rangle,\right.
\end{aligned}
$$

where $\eta_{1}$ and $\eta_{2}$ are the intrinsic parities and the second equation, of course, applies only to identical particles. If the two are identical and $\lambda_{1}=-\lambda_{2}$, then both operators have the same effect, which means that for a state to be an eigenstate of both operators, the parity eigenvalue must be positive for bosons and negative for fermions. Hence we immediately get two of the desired components of the $\gamma \gamma$ state:

$\psi_{M= \pm 2}^{\mathrm{J}^{+}}=\sqrt{\frac{1}{2}}\left[|\vec{P}=0 ; M= \pm 2[W J]+-\rangle+(-1)^{\mathrm{J}} \mid \vec{P}=0 ; M= \pm 2[W J|-+\rangle]\right.$, for which $J \geq 2$. The other two possibilities are easily seen to be

$$
\left.\psi_{M=0}^{J^{ \pm}}=\sqrt{\frac{1}{2}}\left[\frac{1+(-1)^{J}}{2}\right][|\vec{P}=0 ; M=0| W J]++\right\rangle \pm \mid \vec{P}=0 ; M=0[W J|--\rangle]
$$

Note that equations 7.12 and 7.13 immediately yield Yang's theorem, ${ }_{1}^{46}$ which says that a state of two real photons with definite parity cannot have $J=1$. Aleo, for $J=0$ and for all odd parity states, $M=J_{x}=0$ is the only possibility.

Using Eqn. 7.9, the four incoming states can be expanded in terms of the partial waves of equations 7.12 and 7.13 :

$$
\begin{aligned}
& \psi_{J_{x}=0}^{1,2}=\sum_{J} \sqrt{\frac{2 J+1}{3 \pi}} D_{O 0}^{J}(0,0,0)\left[\psi_{M=0}^{J} \pm \psi_{M=0}^{J-}\right] \\
& \psi_{J_{x}= \pm 2}^{2,4}=\sum_{J} \sqrt{\frac{2 J+1}{4 \pi}} D_{ \pm 2 \pm 2}^{J}(0,0,0) \psi_{M= \pm 2}^{J+},
\end{aligned}
$$

where the $D$ functions with the gi:en arguments all actually are unity. Overall the result is simple and obvious, except for the important limitation on $J$ for the heliçity-zero states.

\subsection{The Scattering Cross Section}

Consider the process $a+b \rightarrow c+d$ in the conter-of-mass system. The initial and fral states may be described as in Eqn. 7.5, and with the normalization given in Eqn. 7.6, the density of final states within the phase space $d^{3} p_{c} d^{3} p_{d}$ is

$$
W\left(p_{c} p_{d}\right)=\frac{1}{E_{c} E_{d}} \frac{W}{p} .
$$


Lei $S_{f i}$ be the matrix of scattering amplitudes, and define the transition matrix by $s_{f i}=\delta_{f i}+i \delta^{4}\left(P_{f}-P_{i}\right) T_{f i}$. Then the probability per unit time and volume for the transition is

$$
\begin{aligned}
P & =\frac{1}{V T} \sum_{f \neq i} S_{i f}^{\dagger} S_{f i} \\
& =\frac{1}{(2 \pi)^{4}} \sum_{\lambda_{c} \lambda_{d}} \int \mathrm{d}^{3} p_{c} \mathrm{~d}^{3} p_{d} W\left(p_{c} p_{d}\right)\left|\left\langle c d ; \vec{p}_{c} \vec{p}_{d} \lambda_{c} \lambda_{d}|T| \alpha b ; \vec{p}_{a} \vec{p}_{b} \lambda_{a} \lambda_{b}\right\rangle\right|^{2} \delta^{4}\left(P_{f}-P_{i}\right) \\
& =\frac{1}{(2 \pi)^{4}} \sum_{\lambda_{c} \lambda_{d}} \int\left|\left(c d ; \vec{P}=0 ; \phi \delta^{\prime} p \lambda_{c} \lambda_{d}|T| a b ; \vec{P}=0 ; 00 p \lambda_{a} \lambda_{b}\right\rangle\right|^{2} \mathrm{~d} \Omega
\end{aligned}
$$

The integration over the total momentum has been facilitated by the use of Eqn. 7.7. For the initial state, the =elative flux density is the number of states per unit volume $d^{3} p_{a} d^{3} p_{b}$ times the relative velocity $v_{a}+v_{b}$ :

$$
F=\frac{1}{(2 \pi)^{6}} E_{a} E_{b} \frac{p}{W}\left(v_{a}+v_{b}\right)=\frac{p^{2}}{(2 \pi)^{6}} .
$$

Hence the differential cross section is given by

$$
\frac{\mathrm{d} \sigma}{\mathrm{d} \Omega}=\sum_{\lambda_{c} \lambda_{d}}\left|f_{\lambda_{c} \lambda_{d}}\right|^{2}=\frac{(2 \pi)^{2}}{p^{2}} \sum_{\lambda_{c} \lambda_{d}}\left|\left(c d ; \phi \theta p \lambda_{c} \lambda_{d}|T| a b ; 00 p \lambda_{c} \lambda_{b}\right\rangle\right|^{2} .
$$

Now consider the process $\gamma \gamma \rightarrow \pi x$, where the initial state is as described in the previous section. The final state may be described by Eqn. 7.5, with $\lambda_{1}=\lambda_{2}=0$, and expanded into states of definite isospin $I$ :

$$
\begin{aligned}
& |\pi \pi ; 00\rangle=\sqrt{\frac{2}{3}}\left|\pi^{+} \pi^{-}\right\rangle-\sqrt{\frac{1}{3}}\left|\pi^{0} \pi^{0}\right\rangle \\
& |\pi \pi ; 20\rangle=\sqrt{\frac{1}{3}}\left|\pi^{+} \pi^{-}\right\rangle+\sqrt{\frac{2}{3}}\left|\pi^{0} \pi^{0}\right\rangle,
\end{aligned}
$$

where the notation on the left is $\left|\pi \pi ; I I_{3}\right\rangle$ and the charged pion state is

$$
\left|\pi^{+} \pi^{-}\right\rangle \equiv \sqrt{\frac{1}{2}}|\pi ; 1+1\rangle|\pi ; 1-1\rangle+\sqrt{\frac{1}{2}}|\pi ; 1-1\rangle|\pi ; 1+1\rangle
$$

Define $f_{\lambda}^{I}(s, t)$, where $\lambda=\left|\lambda_{a}+\lambda_{b}\right|$, to be the amplitudes for $\gamma \gamma \rightarrow \pi \pi$, with the normalization as specified in Eqn. 7.18. Assuming no polarization of the incoming photon heams, the cross section for $\gamma \gamma \rightarrow x^{+} x^{-}$is expressed as an average over the two g ossible relative polarizations:

$$
\frac{\mathrm{d} \sigma}{\mathrm{d} \Omega}\left(\gamma \gamma \rightarrow \pi^{+} \pi^{-}\right)=\frac{1}{2}\left|f_{0}^{c}(s, t)\right|^{2}+\frac{1}{2}\left|f_{2}^{c}(s, t)\right|^{2},
$$

where $f_{\lambda}^{c} \equiv \sqrt{\frac{2}{3}} f_{\lambda}^{0}+\sqrt{\frac{1}{3}} f_{\lambda}^{2}$.

Using Eqn. 7.9, these amplitudes are easily expanded into partial waves of definite angular momentum:

$$
\begin{aligned}
& f_{0}^{I}(s, t)=\sum_{J=0}^{\infty}(2 J+1) d_{j 0}^{J}(\theta) f_{J 0}^{I}(s) \\
& f_{2}^{I}(s, t)=e^{-2 i \phi} \sum_{J=2}^{\infty}(2 J+1) d_{20}^{J}(\theta) f_{J 2}^{I}(s),
\end{aligned}
$$

where $f_{J \lambda}^{l}(s) \equiv \frac{2}{\sqrt{b}} T_{J A}^{I}(s)$ and $\left.\left.T_{J A}^{I}(s) \equiv \frac{1}{2}\left\langle\pi \pi_{;} s J I|T| \gamma \gamma_{;} \lambda\right| s J\right] \lambda_{a} \lambda_{b}\right\rangle$. In both of these sums, only even $J$ contribute. That is because, from equations 7.12 and 7.13, the $\gamma \gamma$ states of odd $I$ all have even parity, but the parity of a $\pi \pi$ partial wave is, from Eqn. 7.11, $(-1)^{J}$. Also, the relative phase $e^{-3 i \phi}$ of the two helicity amplitudes is of no consequence for unpolarized beams and is dropped henceforth.

\subsection{DESCRIPTION OF $\pi \pi$ ELASTIC SCATtERING}

Let us apply the same formalism as in the previous section to the process $\pi \pi \rightarrow \pi \pi$. With $g^{I}(s, t)$ defined to be the isospin amplitudes, the partial wave expansion is

$$
g^{I}(s, t)=\frac{1}{p} \sum_{J=0}^{\infty}(2 J+1) A_{J}^{I}(s) P_{J}(\cos \theta)
$$


where $P_{J}(x)$ are the Legendre polynomials. The partial wave amplitudes $A_{J}^{I}$ may be written in terms of phase shifts as

$$
A_{J}^{I}(s)=-\frac{1}{2} i\left[\eta_{J}^{I}(s) \cdot e^{2 i \delta J(s)}-1\right]
$$

For elastic scattering the $\eta_{J}^{I}(s)$ are unity.

Now let us briefly consider some of the general knowledge of $\pi \pi$ partial waves. For $s<1 \mathrm{GeV}^{2}$ and $J \geq 2$, a very good approximation is $\delta_{J}^{J}(s) \approx 0$. For $J=0$ the situation is somewhat confused. The phase shift $\delta_{0}^{0}(s)$ appears to pass through resonance in the region $\sqrt{s}=0.5 \rightarrow 0.7 \mathrm{GeV}$, but there is lack of good dirett evidence for the so-called $\sigma$ resonance at that energy. In any case, the $\delta_{0}^{I}(s)$ are substantial from the $\pi \pi$ threshold on up. ${ }^{\mathbf{7}, 48}$ We will not be concerned with the $\delta_{1}^{I}(s)$, which are dominated by the $\rho$ resonance, but $\delta_{2}^{0}(s)$ above $s=1 \mathrm{GeV}^{2}$ is of particular interest, due to the relatively narrow $f$ resonance at $\sqrt{s}=1.274 \mathrm{GeV}$. These strong-interaction effects have important implications for the process $\gamma \gamma \rightarrow \pi^{+} \pi^{-}$, as may be understood in detail by studying the general unitary and analytic properties of the matrix elements.

\subsection{UNJTARTY, ANALYTJCITY AND THE MODEL FOR $\gamma \gamma \rightarrow \pi^{+} \pi^{-}$}

The scattering matrix $S(s, t, u)$ is believed to be an analytic function of the Mandelstan variables $s, t$, and $u$. Also, it is required by conservation of probability to be unitary: $S^{\dagger} S=1$. In terms of the transition matrix, $T=-i(S-1)$, the unitarity requirement is

$$
i\left(T^{\dagger}-T\right)=T^{\dagger} T
$$

These properties can be used to provide a number of useful constraints on any model of hadronic interactions. A brief review of how that is done is provided here, but for a much more complete discussion, one may refer to Ref. 49.

Consider $T$, for the process $a+b \rightarrow c+d$, as a function of $s$ at a fixed value of $t=t^{\prime}$. All of the singularities in $T$ of the first Rlemann sheet are believed to lie on the real $s$ axis, which is compatible with the unitarity requirement. Considering Eqn. 7.25 , the right hand side requires a sum over all kinematically accesgible channels, so it changes drastically with $s$ as additional channels open. Therefore, there must be a singularity in $T$ at each point $s>\left(m_{a}+m_{b}\right)^{2}$ where a reaction threshold is. Perturbation theory shows that the singularities take the form of cuts with branch points at the thresholds and extending to $s=\infty$, so possible values of $s$ can extend over many Riemann sheets. The sheet containing the real axis below the first branch point is called the physical sheet, and the physical amplitude is defined for real $s=s^{\prime}$ in the s-channel physical region $\left(s>\left(m_{a}+m_{b}\right)^{2}\right.$ and $\left.t<0\right)$ by

$$
T_{a b \rightarrow e d}\left(s^{\prime}, t\right)=\lim _{\epsilon \rightarrow 0} T_{a b \rightarrow c d}\left(s^{\prime}+i \epsilon, t\right) .
$$

Furthermore, if there is a particle with the quantum numbers of $a+b$ but with a mass $m_{0}<m_{c}+m_{b}$, then there is a pole on the real axis at $s=s_{0}=m_{0}^{2}$. The contribution to $T$ of such a pole is called the Born term.

But that is not all. The same analysis may be applied to the crossed reaction $a+\bar{d} \rightarrow e+\bar{b}$, called the $u$-channel. Since with $t$ fixed at $t=t^{\prime}, s$ and $u$ are directly related by the expression $s=\sum m_{i}^{2}-t^{\prime}-u$, then all of the poles an . cuts in the $u$-channel must appear on the unphysical portion of the real $s$ axis, with the cuts extending to $s=-\infty$. Thus the complex $s$ plane looks something like what is shown in Fig. 7.2 .

\subsubsection{The Born Term and Fixcd-t Dispersion Relations}

Let us consider how these generalities apply to $\gamma \gamma \rightarrow \pi^{+} \pi^{-}$. There can be no pole in the s-channel, but certainly there is one in each of the $t$ and $u$ channels, $\gamma \pi^{ \pm} \rightarrow \gamma \pi^{ \pm}$, from single-pion exchange. The residue of the pole is calculated from the first term in a perturbation expansion for a field theory with point coupling of photons to charged spin-zero mesons. Figure 7.3 shows the three relevant Feynman diagrams. A vertex of two pions and one photon contributes to the $S$-matrix a 


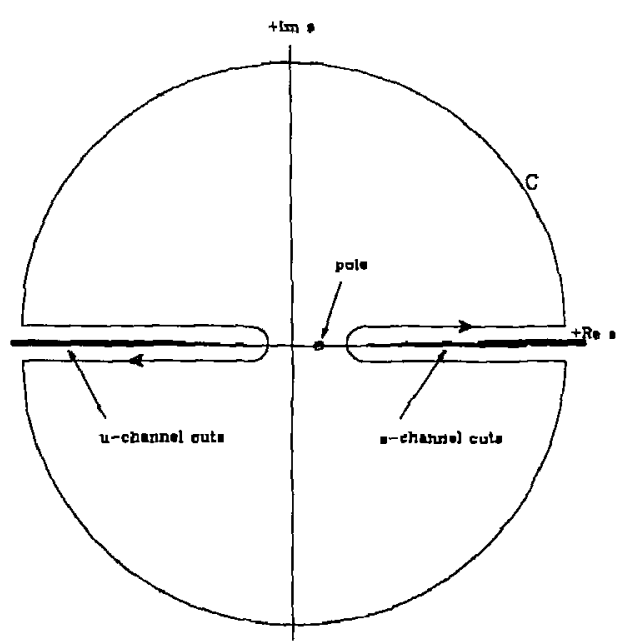

Figure 7.2. The complex $s$ plane for the transition matrix $T(s, t)$ at constant $t=t^{\prime}$. The contour $C$ is that used to derive the fixed- $t$ dispersion relations.

fartur $-i e\left(p+p^{\prime}\right)_{\mu,}$ where $p$ and $p^{\prime}$ are the pion momenta and $\mu$ is the Lorentz component of the photon? 14 The vertex of two pions and two photons contributes $2 i e^{2} g_{\mu \nu}$, and the meson propagator is $i /\left(p^{2}-m_{\pi}^{2}\right)$. Otherwise the calculation praceeds as in QED, and the result is ${ }^{50}$

$$
\frac{\mathrm{d} a}{\mathrm{~d} \Omega}=\frac{1}{2}\left|B_{0}\right|^{2}+\frac{1}{2}\left|B_{2}\right|^{2}
$$

helieity $-0: \quad B_{0}=\frac{\alpha}{W} \sqrt{\beta} \frac{1-\beta^{2}}{1-\beta^{2} \cos ^{2} \theta} \equiv \frac{1}{2 \pi} \sqrt{\beta s} F_{0}^{B}(s, t)$

helicity-2: $\quad B_{2}=\frac{\alpha}{W} \sqrt{\beta} \frac{\beta^{2} \sin ^{2} \theta}{1-\beta^{2} \cos ^{2} \theta} \equiv \frac{\sqrt{\beta}}{2 \pi} \frac{t u-m_{\pi}}{\sqrt{s}} F_{2}^{B}(s, t)$,

where $\beta=\sqrt{\left(s-4 m_{n}^{2}\right) / s}$ is the pion velocity. The reduced amplitudes $F_{0}$ and $F_{2}$ are defined such that they are free of all kinematic eingularities ${ }^{61}$ and may be
144
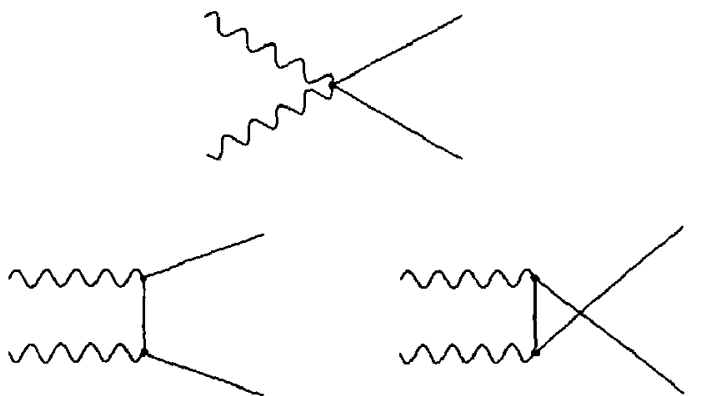

Figure 7.3. The Feynman diagrams for the Born term in the proces $\gamma \gamma \rightarrow \pi^{+} \pi^{-}$.

written in terms of the Mandelstam variables as

$$
\begin{aligned}
& F_{0}^{B}=2 \pi \alpha \frac{m_{\pi}^{2}}{\left(m_{\pi}^{2}-t\right)\left(m_{\pi}^{2}-u\right)} \ldots . \\
& F_{2}^{B}=2 \pi \alpha \frac{1}{\left(m_{\pi}^{2}-t\right)\left(m_{\pi}^{2}-u\right)} .
\end{aligned}
$$

To include formally the contributions of the cuts, fixed $t$ dispersion relations may be used. Figure 7.2 shows an integration contour $C$ in the complex $s$ plane. Consider the istegral

$$
\int_{C} \frac{F\left(s^{\prime}, t^{\prime}\right) d s^{\prime}}{s^{\prime}-s}=2 \pi i\left[F\left(s, t^{\prime}\right)-F^{B}\left(s, t^{\prime}\right)\right],
$$

which has been evaluated by using Cauchy's theorem and including the residue of the single pole of $F$ within the contour. By letting the arcs of the contours go to infinity, the integral may be evaluated from only the contributions along the real axis. Using the property of hermitian analyticity, $T(s+i \epsilon, t)=T^{\bullet}(s-i c, t)$, results in the dispersion relations

$$
F_{0,2}\left(s, t^{\prime}\right)=F_{0,2}^{B}\left(s, t^{\prime}\right)+\frac{1}{\pi} \int_{m_{\frac{2}{\pi}}}^{\infty} \frac{\Im F_{0,2}\left(u^{\prime}, t^{\prime}\right) d u^{\prime}}{u^{\prime}-u}+\frac{1}{\pi} \int_{4 m_{\pi}^{2}}^{\infty} \frac{\Im F_{0,2}\left(s^{\prime}, t^{\prime}\right) d s^{\prime}}{s^{\prime}-s},(7.30)
$$


where the first of the two integrals has been rewritten as an integration over $u^{\prime}=2 m_{\pi}^{2}-t^{\prime}-s^{\prime}$ and $\Im$ denotes the imaginary part. The two integrals, which are the contributions respectively of the left hand and right hand cuts of Fig. 7.2, are defined for real s by approaching the real axis fror above. They are exceedingly complicated, so the equations cannot be solved without making some explicit assumptions about the form of $\$ F(s, t)$ in the region of integration.

\subsubsection{Resonant Partial Waves}

The approach to be taken is to ronsider only contributions from exchanges, in the $s$ or $u$ changels, of single resonances. Since a resonance has definite spin and parity, then only a single partial wave must be considered. The analytic propertics of a partial wave amplitude follow directly from those of the full amplitude and the $D_{\lambda_{1}, \lambda_{2}}^{J}$ funetions. ${ }^{52}$ In particular, there are cuts along the positive real $s$ axis extending from the first threshold to $s=\infty$. Since there is no fundamental difference between a resonance and a stable hadron besides the mass (assuming all other quantum numbers to be the same), then one expects the exchange of a resonance to result in a pole in the complex s plane near the real axig, but above the first branch point. 'th pole can neither be on the real axis nor on the physical sheet, so hermitian analyticity dem.. Ts that there be in fact two poles, placed symmetrically on opposite sides of the real axis .. nd on an unphysical sheet. Only the one which is below the real axis is close to the physical shert -ince the unphysical sheet is reached from the physical sheet by crossing the real axis tw. above. Assuming that this pole, located at $s_{r}=m_{r}-i m_{r} r$, dominates the behavior of $T_{J}(s)$ for $s$ near $s_{r}$, so only the first term of a series expansion of $T_{J}(s)$ about $s=s_{r}$ need be kept, gives the relativistic Breit-Wigner shape for the resonance:

$$
T_{J}(s)=\frac{m_{\mathrm{r}} \Gamma}{m_{r}^{2}-s-i m_{r} \Gamma} .
$$

Such a formula may be improved by including contributions from other singularities. In particular, consider the branch point at the two-particle threshold $s_{i}=\left(m_{a}+m_{b}\right)^{2}+\uparrow$ If the branch point is not too close, then the amplitude can be approximated by the first terms in an effective range expansion about $s_{t}, 52$ This results in an energy dependence for the width $\Gamma$. In particular, $r$ contains a threshold factor $p^{2 J+1}$. In Ref. 53, Lyth auggests the parameterization, for $f \rightarrow \pi \pi$,

$$
\begin{aligned}
& \Gamma(s)=\Gamma_{0}\left(\frac{p}{p_{0}}\right)^{5}\left(\frac{s_{0}}{s}\right)^{\frac{1}{2}}\left(\frac{s_{0}+a}{s+a}\right)^{2} ; \\
& p \equiv \frac{1}{2} \sqrt{s-4 m_{\pi}^{2}}, \quad p_{0} \equiv \frac{1}{2} \sqrt{s_{0}-4 m_{\pi}^{2}}
\end{aligned}
$$

where $s_{0}=m_{f}^{2}$ and $a \sim m_{f}^{2}$. This is the form assumed in the remainder of this analysis. For the $f$ resonance,

$$
m_{r}=m_{f}=1.274 \pm 0.005 \mathrm{GeV}, \quad \Gamma_{0}=\Gamma_{f}=0.178 \pm 0.020 \mathrm{GeV}
$$

and we use $a=0.5 \mathrm{GeV}^{2}$. The resulting $\pi \pi$ phase shift for the $I=0$, $J=2$ amplitude is given by $\tan \delta_{2}^{0}(s)=m_{f} \Gamma(s) /\left(m_{f}^{2}-s\right)$, and this gives a fayorable comparison with measurements from pion-nucleon scattering, as shown in Fig. 7.4. 54

Another modification of Eqn. 7.31 is necessary to include inelastic effects. Note that it is only the position of the pole which is characteristic of the resonance itself, and that is all which remains constant for the resonance when observed in reveral different reactions. What needs modified is the residue. That depends on the coupling of thr resonance to the initial and final states. It can be factored into a product of two partiol widths, of which one depends on only the initial state and the other on only the final state. For example, for $\gamma \gamma \rightarrow f \rightarrow \pi^{+} \pi^{-}$, the

I In general there could be several such thresholde. For example, the $f$ resonance in affected by the $\pi \pi, 4 \pi$, and $K K$ thresholds. We neglect the Jatter two, because the branching ratios of the f to those channels are relatively amall. 


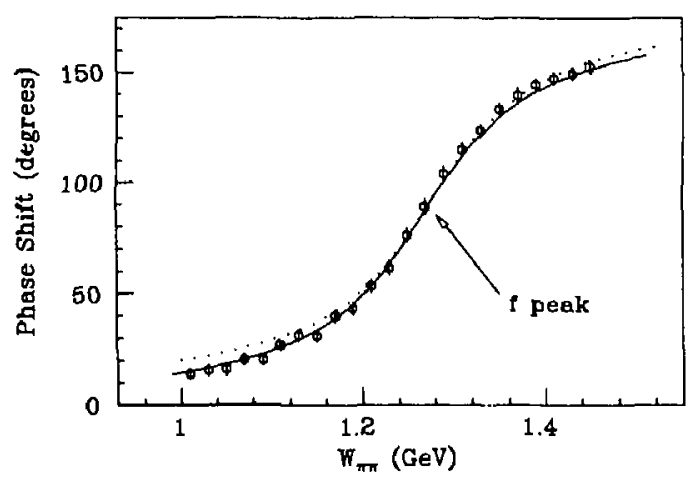

Figure 7.4. The $I=0, J=2: i \pi$ phase shift as measured from pion-nucleon scattering. The solid curve is the best fit, by varying the parameter $a$, of Eqn. 7.32, for which $a=0.5 \mathrm{GeV}^{2}$. The dotted curve shows the Breit-Wigner shape with no energy dependence for the width.

appropriate expression for the amplitude is

$$
\begin{aligned}
T_{2}^{\epsilon}(s)=\sqrt{\frac{2}{3}} T_{2 \lambda}^{0}(s) & =\frac{m_{f}\left[\frac{2}{3} \Gamma_{\gamma \gamma}^{\lambda}(s) \Gamma_{\pi \pi}(s)\right]^{\frac{1}{2}}}{m_{f}^{2}-s-i m_{f} \Gamma(s)}, \\
\text { where } \Gamma_{\gamma \gamma} & =\operatorname{BR}(f \rightarrow \gamma \gamma) \cdot \Gamma \\
\text { and } \Gamma_{\pi \pi} & =\mathrm{BR}(f \rightarrow \pi \pi) \cdot \Gamma .
\end{aligned}
$$

The factor of $\sqrt{\frac{2}{3}}$ is from Eqn. 7.19, and $\lambda$ denotes the fact that there are separate amplitudes for each of the two possibie $\gamma \gamma$ helicities. The $s$ dependence of $\Gamma_{\pi \pi}(s)$ should be the same as that of $\Gamma(s)$, since $\pi \pi$ is by far the dominant decay mode. For the $s$ dependence of $\Gamma_{\gamma \gamma}(s)$ we assume the same form as in Eqn. 7.32, except that $m_{\pi}$ is replaced by $m_{\gamma}=0$.

A little reflection on the meaning of the constants $m$, and $\Gamma_{f}$ is in order. Ideally they should give the position of the resonance pole as $s_{r}=m_{f}^{2}-i m_{f} \Gamma_{f}=$
$1.62-0.227 i$, since that is the quantity, given in Ref. 6 , which characterizes the resonance. However, when the form in Eqn. 7:32 is used for the width, then the pole moves to the position $s_{r}=1.59-0.225 i$, which is a change of the order of one percent. Changing $m$, from $1.274 \mathrm{GeV}$ to $1.284 \mathrm{GeV}$ is enough to return the pole to the position given in Ref. 6, but one must keep in mind that all experiments from which those measurements were taken had to do a similar sort of extrapolation to determine the pole position. Hence there is a lot of potential for $1 \%$ errors caused by the parameterization of the resonance shape. $\uparrow$

\subsubsection{Estimate of Contributions from the Left Hand Cut}

Now let us use the parameterizations for resonance exchange to estimate possible contributions from the left hand cut in Eqn. 7.30. To do so, we consider the reaction $\gamma \pi \rightarrow \gamma \pi$ (these calculations are by Lyth in Ref. 51). Using the inverse of Eqn. 7.9 with $\lambda_{\gamma}= \pm 1$ and $\lambda_{\pi}=0$ gives the description of the $\gamma \pi$ state, which leads to the partial wave expansions for the amplitudes, aside from overall phases of $e^{ \pm \phi}$, of

$$
T_{ \pm}(s, t)=2 \sum_{J=0}^{\infty}\left(\frac{2 J+1}{4 \pi}\right) d_{1 \pm 1}^{J}(\theta) T_{J \pm}(s),
$$

where the + or - refers to the relative helicity of the photons.

Consider the contribution of vector resonances to the $J=1$ partial wave. From Eqn. 7.11, one can see that a $J=1$ partial wave with negative parity is formed by the combination $T_{1+}(s)-T_{1-}(s)$, so the resonance must couple equally to the two helicities if parity is to be conserved. Hence

$$
T_{1+}(s)=T_{1-}(s)=\frac{\frac{1}{2} m_{r} \Gamma_{7 \pi}}{m_{r}^{2}-s-i m_{r} \Gamma_{r}}
$$

$\uparrow$ Note that the change in $m_{f}$ is significant, eince it is greater than the error quoted for $m_{f}$ in Ref. B. However, even that error estimate is said to be no more than an educated guess. 
When the pion mass and the finite resonarce width are neglected, then the imaginary parts of the $J=1$ resonant contributions to Eqn. 7.35 are

$$
\begin{aligned}
& \Im T_{-}(s, t)=-\frac{3}{4 \pi} \frac{\Gamma_{\gamma_{\pi}}}{m_{r}} t \delta\left(m_{r}^{2}-s\right) \\
& \Im T_{+}(s, t)=-\frac{3}{4 \pi} \frac{\Gamma_{\gamma \pi}}{m_{r}} u \delta\left(m_{r}^{2}-s\right) .
\end{aligned}
$$

These relations are rewritten in terms of the reduced amplitudes (see Eqn. 7.27), after which crossing symmetry is used to give the corresponding amplitudes for the reaction $\varphi \uparrow \rightarrow \pi \pi . T_{-}$becomes the helicity-zero amplitude and $T_{+}$the helicity-two amplitude:

$$
\begin{aligned}
& \Im F_{0} \approx \delta \pi^{2} \frac{\Gamma_{\gamma \pi}}{m_{r}} \delta\left(u-m_{r}^{2}\right) \\
& \Im F_{2} \approx 6 \pi^{2} \frac{\Gamma_{\gamma \pi}}{m_{r}} \frac{1}{m_{r}^{2}} \delta\left(u-m_{r}^{2}\right) .
\end{aligned}
$$

Inserting these into the first integral of Eqn. 7.30 yields the estimates for the resonance contributions to the left hand cuts:

$$
\begin{aligned}
& F_{0}^{*}=6 \pi \frac{\Gamma_{\gamma \pi}}{m_{r}} \frac{1}{m_{r}^{2}-u} \\
& F_{2}^{r}=6 \pi \frac{\Gamma_{\gamma \pi}}{m_{r}} \frac{1}{m_{r}^{2}} \frac{1}{m_{r}^{2}-u} .
\end{aligned}
$$

In Ref. 51, these expressions are used to estimate the u-channel resonance contribution relative to the Born term. The result is that the resonance terms are completely negligible near the $\pi \pi$ threshold but may be significant in the region near one $\mathrm{GeV}$ for large-angle scattering. But that estimate is very conservative, using $\Gamma_{\gamma \pi} / m_{r} \approx 0.1 \alpha$, which is about eight times greater than the experimental value now found in Ref. 6 for the $\rho$. For the helicity-zero amplitude, the contribution from $\rho$ exchange may oe as much as half the Born contribution in the $f$ region at large angles. But that is not important since even the helicityzero Born term is negligible in that region, compared with the helicity-two Born term. Figure 7.5 shows the effect of $\rho$ exchange in the helicity-two amplitude. At

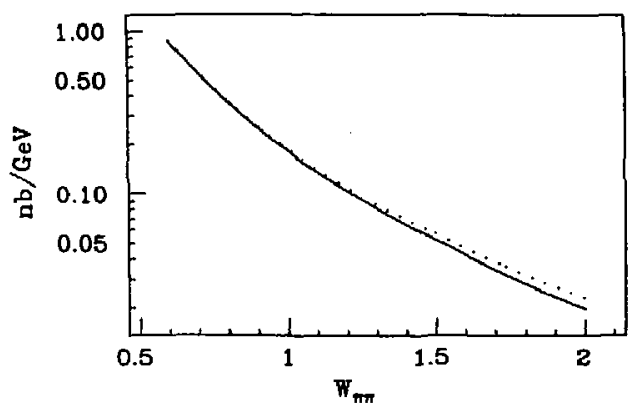

Figure 7.5. The effect of $p$ exchange on the $\pi^{+} \pi^{-}$continuum in $e^{+} e^{-}-$ $e^{+} e^{-} \pi^{+} \pi^{-}$. The solid curve shows the contribution from single pion exchange, while the dotted curve includes the p-exchange contribution. The photon flux is calculated from EPA, and the final pion-pair state is integrated over the acceptance defined by $-0.6 \leq \cos \theta \leq 0.6$ and $k_{\perp} / \boldsymbol{W} \leq \mathbf{0 . 2}$.

$W=0.6 \mathrm{GeV}$ theie is an increase of only $0.5 \%$ over the Born term; at $W=m$ f the increase is $5.3 \%$; at $W=2.0 \mathrm{GeV}$ the increase is $17 \%$. Thus the $\rho$-exchange contribution is almost completely negligible compared with pion exchange.

The $\omega$, which has a relatively large $\gamma \pi$ width, does not contribute to the charged channel, and one expects the heavier vector mesons, such as the $A_{1}$, to contribute considerably legs than the $p_{1}$ since their masses place them further from the physical region. Also, higher partial waves give even more negligible contributions, so all indications point to conplete dominance of the Born term in the $t$ and $u$ channels.

\subsubsection{Corrections Required by Unitarity}

It is a useful exercise to write explicitly Eqn. 7.25, the unitarity relation, for the process $\gamma \gamma \rightarrow \pi \pi$. To simplify the discussion, it is best actually to work with the pure isospin states of Eqn. 7.19. Let $A^{I}\left(\phi \theta ; \phi^{\prime} \theta^{\prime} ; s\right)$ be the amplitude for elastic 
$\pi \pi$ scattering in the center-of-mass system from the initial angles $\left(\phi^{\prime}, \theta^{\prime}\right)$ to the final angles $(\phi, \theta) . T,(\phi \theta ; s)$ is the amplitude for photons incoming along the $z$ axis and pions outgoing in the directions given in the center-of-mass system by the angles $(\phi, \theta)$. Amplitudes for purely QED processes like $\gamma \gamma \rightarrow \gamma \gamma$ are small compared with hadronic amplitudes, so any term including them may safely be neglected. Then the unitarity relation is (with the isospin index $I$ suppressed)

$$
\begin{aligned}
i\left[T_{\lambda}^{\dagger}(\phi \theta ; s)-T_{\lambda}(\phi \theta ; s)\right]=\int & \mathrm{d}^{3} p_{1}^{\prime} \mathrm{d}^{3} p_{2}^{\prime} W\left(p_{1}^{\prime} p_{2}^{\prime}\right) \\
& \times A^{\dagger}\left(\phi \theta ; \phi^{\prime} \theta^{\prime} ; s\right) T_{\lambda}\left(\phi^{\prime} \theta^{\prime} ; s\right) \delta^{4}\left(p_{1}^{\prime}+p_{2}^{\prime}\right) \\
& + \text { inelastic contribútions, }
\end{aligned}
$$

where $p_{i}^{\prime}$ refers to one pion of the pair crstesponding to the angles $\left(\phi^{\prime}, \theta^{\prime}\right)$. The only significant inelastic contributions to Eqn, 7.40 are those coraing from strong interaction processes, such os $\gamma \mathcal{Y} \rightarrow K^{+} K^{-}$, and of those, only the ones energetically accessible are included, depending on the value of $s$.

Now we may use Eqn. 7.7 to transform to the center-of-mass variables $\left(p^{\prime}, \phi^{\prime}, \theta^{\prime}\right)$ and integrate out the $\delta^{\mathbf{4}}\left(p_{1}^{\prime}+p_{2}^{\prime}\right)$. Also, let us substitute in the partial wave expansions of Eqn, 7.22 for $T_{\lambda}^{I}\left(\phi \theta_{i} s\right)$, using the definitions $f_{\lambda}^{J} \equiv \frac{2 \pi}{p} T_{\lambda}^{f}$ (Eqn. 7.18) and $f_{J \lambda}^{J} \equiv \frac{2}{p} T_{J \lambda}^{\prime}$ (Eqn. 7.22). For $A^{I}\left(\phi \theta ; \phi^{\prime} \theta^{\prime} ; s\right)$ we substitute the expansion of Eqn. 7.23, suitably rotated:

$$
A^{I}\left(\phi \theta ; \phi^{\prime} \theta^{\prime} ; s\right)=2 \sum_{J=0}^{\infty} \sum_{M=-J}^{J}\left(\frac{2 J+1}{4 \pi}\right) d_{M 0}^{J}(\theta) d_{M 0}^{J}\left(\theta^{\prime}\right) e^{i M\left(\phi-\phi^{\prime}\right)} \mathcal{A}_{J}^{I}(s) .
$$

A et these substitutions, the angular integrations become trivial, and we are left wi h s separate requirement for each partial wave:

$$
s T_{J \lambda}^{I}(s)=\left[A{ }_{J}(s)\right]^{\bullet} T_{J \lambda}^{I}(s)+\text { inelastic contributions. }
$$

If the inelastic contributions are negligible or zero, as they must be for small enough s, then Eqn. 7.42 may be solved. Uaing Eqn. 7.24, one finds

$$
T_{J \lambda}^{f}(s)=\left|T_{J_{\lambda}}^{Z}(s)\right| e^{i \delta} J(s)
$$

This is equivalent to Watson's theorem for photoproduction, ${ }^{55}$ and essentially says that the phase of the $\gamma \gamma \rightarrow \pi \pi$ amplitude is given by the $\pi \pi$ phase ahift if the energy is low enough that no significant inelastic channels are open. One can consider this phase to be due to final-state interactions of the pions after they are produced from the photons.

\subsubsection{Justification of the Born Approximation}

From the discussion of Section 7.3, below the $f$ region the only aignificant phase shifts are $\delta_{0}^{I}$. There are no ineinstic contributions in that region, so Eqn, 7.43 must be considered to be exact. In Kef. 51, the rough mesurements available for $\delta_{0}^{f}$, along with the fixed-t disperaion relations of Eqn, 7.30, are used to calculate the necessary corrections to $T_{00}^{I}(s)$, and one finds that, although large corrections ( $\approx 30 \%$ enhancement) are necessary near threshold, from non-resonant effects, they become negligible above $\sqrt{8}=0.4 \mathrm{GeV} .^{56}$ When nearing the $f$ region, the phase shifts of the $\delta_{2}^{0}$ partial wave begin to become signiafcant. We will see that, to a good approximation, Eqn. 7.43 must be satisfied even throughout the $f$ region, up to about $1.4 \mathrm{GeV}$, leading to corrections to a simple model of the Born term combined with a resonant amplitude.

Such corrections, though, are well defined by Eqn. 7.43. Furthermore, we have shown that contributions from vector meson exchanges are negligible with respect to the pion exchange, so the Born term, with unitazity corrections, is all that is needed to describe the continuum production itself. The remaining question concerns to what extent the Born term actually is described by the diagrams of Fig. 7.3. We have assumed that the pions are point particles. That 
seems phyaically reasonable for low enough photon energy, since the actual size of the pion will be small compared with the photon wavelength. $A$ fairly rigorous justification for the applicability of the point-coupling calculation near threshold may be arrived at through the hypothesis of a partially conserved axial current (PCAC) and current algebra, ${ }^{57}$ which are theoretical ideas that have met with much success in describing phenomena of low energy pion physics. The result is that the point-like coupling is valid up to the addition of terms with a relative magnitude of approximately $s / m_{p}^{2}$. In fact, we will see that a good description of the data is achieved for the entire region below the $f$, which agrees with the typical theoretical prejudice that such approximations can be expected to be good up to energies of about $1 \mathrm{GeV}$.

The Born cross section falls like $1 / s$, whereas from Eqn. 7.2 we expect at high energies that the cross section must fall a $/ s^{3}$, due to the $1 / \mathrm{s}$ behavior of the form factor. However, since the Born term agrees well with the data below the $f$, it is reasonable to use it as an extrapolation through the full range of the $f$, up to about $1.4 \mathrm{GeV}$. It only is necessary to make an estimate of what errors this extrapolation might cause for the measurement of the resonant part of the cross section.

\subsubsection{Constraints on the Coupling of $\gamma \gamma$ to the f Resonance}

We have determined that for the region well below the $f$ resonance and above $\sqrt{s}=0.4 \mathrm{GeV}$, the cross section should be adequately represented by the Born term alone, unless there happens to be some large direct coupling of $\gamma \gamma$ to lowmass scalar resonances. For the region near the $f$, the resonant contribution to the $J=2, I=0$ partial wave must be included.

In general there are two amplitudes to consider for the $f$, with one for each possible helicity. However, in Chapter 1 we have seen that there is considerable evidence that the helicity-two amplitude is strongly dominant. For now, let us assume zero coupling for helicity-zero, so the cross section is written, for $\gamma \gamma \rightarrow \pi^{+} x^{-}, 29$

$$
\frac{\mathrm{d} \sigma}{\mathrm{d \Omega}}=\frac{1}{2}\left|B_{0}(s, t)\right|^{2}+\frac{1}{2}\left|B_{2}(s, t)+5 \sqrt{\frac{2}{3}} d_{2 \mathrm{o}}^{2}(\theta) R_{22}^{0}(s)\right|^{2},
$$

where the $B_{\lambda}$ are as defined in Eq. 7.27, and the $I=0$ resonance contribution is defined as in Eqn. 7.34:

$$
R_{22}^{0}(s)=\frac{2}{\sqrt{s}} T_{22}^{0}\left(s:=\frac{2}{\sqrt{s}} \frac{g}{m_{f}^{2}-s-i m_{f} \Gamma(s)} ; \quad g \equiv m_{f}\left[\Gamma_{\gamma \gamma} \Gamma_{\pi \pi}\right]^{\frac{1}{2}}\right.
$$

Suppose that the inelastic channels still are small in the $f$ region. Then the $J=2$ partial wave should satisfy the unitarity constraint of Eqn. 7.43. It is interesting to see what that implies for the phase of $g$ in Egn. 7.45. $†$ The $J=2$ component of $B_{2}(s, t)$ is

$$
\begin{aligned}
\frac{2}{\sqrt{s}} B_{22}(s) & =\frac{1}{2} \int_{-1}^{1} B_{2}(s, t) d_{20}^{2}(\theta) \mathrm{d} \cos \theta \\
B_{22}(s) & =\frac{\alpha}{4} \sqrt{\frac{3 \beta}{2}}\left[\frac{\left(1-\beta^{2}\right)^{2}}{2 \beta^{3}} \ln \left(\frac{1+\beta}{1-\beta}\right)-\frac{1}{\beta^{2}}+\frac{5}{3}\right] .
\end{aligned}
$$

Consider the sum of the $I=0, J=2$ amplitudes, $\sqrt{2 / 3} B_{22}(s)+T_{22}^{0}(s)$, for $s=m_{f}^{2}$. Equation 7.43 requires that the sum have a phase of $e^{i \pi / 2}$ at that energy, so setting the real part of the sum to zero constrains the imaginary part of $g$;

$$
\begin{aligned}
\Im g & =\sqrt{\frac{2}{3}} m_{j} \Gamma B_{22}\left(m_{f}^{2}\right) \\
& =0.00025 \mathrm{GeV}^{2} .
\end{aligned}
$$

As tor the real part of $g$, the experimental data definitely require $\mathbb{R} g>0$, since the $f$ peak is shifted downwards in $\sqrt{s}$ relative to its position when observed without interference with the Born term.

$\uparrow$ This analysis closely follows that of Ref. 50 . 
Equation 7.47 essentially means that the sum of the $I=0, J=0$ amplitudes may be written as

$$
A_{22}^{0}(s)=\sqrt{\frac{2}{3}} B_{22}(s)+T_{22}^{0}(s)=\frac{g^{\prime}}{m_{j}-s-i m j \Gamma(s)}
$$

with $g^{\prime} \equiv \Re g+\sqrt{2 / 3}\left(m_{f}^{2}-s\right) B_{22}(s)$. This has the form of a resonance pole with an energy dependent coupling $g^{\prime}$ and explicitly satisfies Eqn. 7.43. The difference here from a more typical resonance amplitude is that $g^{\prime}$ has a relatively rapid variation across the resonance, due to the $1 / \sqrt{s}$ energy dependence of the Born amplitude. This phenomenon causes difficulties when measuring the $\gamma \gamma$ width of the $f$ from charged pion pairs. The problem results both because of the large resonance width $\Gamma$ and because of the, latge $J=2$ contribution from the Born term. If either of those quantities were small, then from Eqn. 7.47 it is clear that $g$ would be essentially real and there would be no issue about whether the energy dependence is properly described.

The process $\gamma \gamma \rightarrow f \rightarrow \pi^{0} \pi^{0}$ has no Born term from pion exchange, so one expects that the Crystal-Ball measurement does not suffer from any serious theoretical ambiguities. Therefore, assuming that their measurement of the $\pi^{0} \pi^{0}$ spectrum yields a value for the magnitude of $g$, as defined above, then Eqn. 7.47 may be used to predict the phase of $g$. The Crystal-Ball result, $\Gamma_{\gamma \gamma}=2.7 \pm 0.2 \pm 0.6 \mathrm{keV}$ (assuming only helicity-two coupling) yields $|g|=$ $0.00080 \mathrm{GeV}^{2}$, and hence we predict a phase for the coupling of $\gamma \gamma$ to the $f$ of $\phi=\sin ^{-1}(\vartheta g /|g|) \approx 0.32$ radians.

\subsubsection{Further Consideration of the Unitarity Condition}

The prediction for the phase of the $\gamma \gamma \rightarrow \pi \pi$ amplitude relies upon the assumption, implicit in Eqn. 7.43, that the contributions from other inelastic processes in the $J=2, I=0$ partial wave are negligible. The validity of this assumption can be checked by a method due to Lukaszuk 58 He writes the inelastic contributions to Eqn. 7.42 as $\sum_{n \neq \pi \pi} a_{n} b_{n}^{*}$, where $a_{n}$ is the $J=2$, $I=0$ partial wave for $\gamma \gamma \rightarrow n$, and $b_{n}$ is the corresponding partial wave for $\pi \pi \rightarrow n .+$ Only states $n$ which are hadronic and kinematically accessible at the energy $\sqrt{s}$ are included in the sum. In this case, for $s=m_{f}^{2}$, the possibilities are $n=\{K K, 4 \pi, K \pi \pi, \eta \eta, \eta \pi \pi\}$, of which only the first two have been measured in two-photon scattering.

First let us consider how the inelasticity $\eta_{J}^{J}$ of Eqn. 7.24 is related to the reaction cross section. The result of squaring Eqn. 7.23 and integrating over the full solid angle is the total elastic cross section

$$
\sigma_{\pi \pi \rightarrow \pi \pi}^{I=0}=\int\left|g^{0}(s, t)\right|^{2} \mathrm{~d} \Omega=\frac{4 \pi}{s-s_{0}} \sum_{J}(2 J+1)\left|\eta_{J}^{0}(s) \cdot e^{2 i \sigma_{j}^{0}(s)}-1\right|^{2},
$$

where $s_{0} \equiv 4 m_{\pi}^{2}$. The reaction or inelastic part of the cross section then is identified as

$$
\sigma_{r}^{I=0}=\frac{4 \pi}{s-s_{0}} \sum_{J}(2 J+1)\left[1-\left(\eta_{J}^{0}\right)^{2}\right],
$$

from which it follows that the $b_{n}$ have the property

$$
\sum_{n \neq \pi \pi}\left|b_{n}\right|^{2}=\frac{1}{4}\left[1-\left(\eta_{2}^{0}\right)^{2}\right]
$$

Similarly, the total $I=0$ cross section for $\gamma \gamma \rightarrow \pi \pi$ may be calculated:

$$
\sigma_{\lambda}^{I}=\int\left|f_{\lambda}^{I}\right|^{2} \mathrm{~d} \Omega=\frac{16 \pi}{s} \sum_{J}(2 J+1)\left|T_{J \lambda}^{I}\right|^{2} .
$$

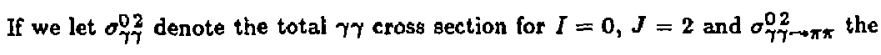
part of that cross section into two pions, then the $a_{n}$ have the property

$$
\sum_{n \neq \pi \pi}\left|a_{n}\right|^{2}=\frac{s}{80 \pi}\left(\sigma_{\gamma \gamma}^{02}-\sigma_{\gamma \gamma \rightarrow \pi \pi}^{02}\right)
$$

f Actually, Lukassuk considers the form factor for the process $e^{+} e^{-} \rightarrow \pi \pi$, but the calculation is closely analagous to that presented here. 
After substituting Eqn. 7.24 and $T_{22}^{0}(s) \equiv\left|T_{22}^{0}(s)\right| e^{i \psi(a)}$, then Eqn. 7.42 may be written as

$$
\left|T_{22}^{0}\right|\left(\frac{\eta_{2}^{0} \exp \left[2 i\left(\psi-\delta_{2}^{0}\right)\right]-1}{2 i}\right)=e^{i \psi} \sum_{n \neq \pi \pi} a_{n} b_{n}^{0}
$$

Lukaszuk's bound now follows from taking the absolute value squared of this equation and applying Schwartz's inequality to the right hand side:

$$
\left|T_{22}^{0}\right|\left[\frac{1}{4}\left(1-\eta_{2}^{0}\right)^{2}+\eta_{0}^{2} \sin ^{2}\left(\psi-\delta_{0}^{2}\right)\right] \leq \sum_{n \neq \pi \pi}\left|a_{n}\right|^{2} \sum_{n \neq \pi \pi}\left|b_{n}\right|^{2}
$$

Using equations $\mathbf{7 . 5 1}$ and $\mathbf{7 . 5 3}$, this may be written in the convenient form

$$
\frac{1}{4}\left(1-\eta_{2}^{0}\right)^{2}+\eta_{0}^{2} \sin ^{2}\left(\psi-\delta_{0}^{2}\right) \leq \frac{1}{4}\left[1-\left(\eta_{2}^{0}\right)^{2}\right] \cdot[r-1]
$$

where $r \equiv \alpha_{\gamma \gamma}^{02} / a_{\gamma \gamma \rightarrow \pi * *}^{02}$

Now, at $s=m_{f}^{2}$, the inelasticity is known to be $\eta_{2}^{0}\left(m_{f}^{2}\right)=\Gamma_{\pi \pi} / \Gamma=0.84$, so we have the limit $\sin ^{2}\left(\psi-\delta_{2}^{0}\right) \leq 0.088(r-1)-0.0076$. In Ref. 53, Lyth uses the present-day results from $\gamma \gamma$ physics to estimate $r$ and arrives at the result $r-1<60 \mathrm{nb} / 240 \mathrm{nb}=0.25$, which in turn implies that the phase of the $J=2$, $I=0$ amplitude for $\gamma \gamma \rightarrow \pi \pi$ must be within about 0.1 radians of the value of the corresponding $\pi \pi$ phase shift. Relating this to the analysis of the previous section, we find that the imaginary part of $g$ must be within the range

$$
\Im g=0.00025 \pm 0.00007 \mathrm{GeV}^{2}
$$

and the phase of the $\gamma \gamma f$ coupling, assuming the Crystal Ball result for $\Gamma_{\gamma \gamma}$, is predicted to be in the range $\phi=0.32 \pm 0.10$.

\subsection{THE MODEL OF MENNESSIER}

Mennessier has produced a complete model for meson pair production from two photons ${ }^{59}$ which is similar to the model presented in the previous section but considerably more complicated in some respects. He begins with an effective Lagrangian, so there is the usual pion-exchange Born term, but also additional vector-meson exchanges. The vector-meson exchanges, which give contributions to the left hand cut in the complex $s$ plane, are determined from the crossed channel reactions, so the coupling strensths of the vector mesons to $\pi \gamma$ are taken from the known radiative partial widths. That is the same approach taken in Section 7.4.3, except that there the effects of the crossed channels are estimated from the fixed-u dispersion relations, while Mennessier introduces terms such as $\varepsilon_{\mu \nu \sigma \rho} h_{\rho} \ddot{\pi} \cdot\left[\partial_{\mu} \vec{\rho}_{\nu}-\partial_{\nu} \vec{\rho}_{\mu}\right] F_{\Delta \rho}$ into the Lagrangian density. $\dagger$ Although such point couplings often work well for single pion exchange at sufficiently low energy, it is well known that form factors must be introduced, when using such a formalism for the heavier mesons, in order to describe hadronic scattering data. Mennessier himself states in Ref. 59 that his model must overestimate even the $\rho$-exchange contribution. Therefore, the analysis of Section 7.4.3, which is due to Lyth, may be more correct. There, we saw thist the p-exchange contribution is negligible when compared with pion exchange, so when considering the Mennessier model, we always will neglect all of the vector-meson exchanges. Only pion exchange is included for the $t$ and $u$ channels.

Corrections required by unitarity are made for the $t$ and u-channel contributions through an analytic, coupled channel $K$-matrix formalism for final state interactions, in which both pion and kaon intermediate states are included. All partial waves for the Born term are included, but only the $S$ and $D$ waves are unitarized. The solutions for these lowest partial waves of the $K$-matrix formaism arc obtained by fitting to available hadronic scattering data. It is interesting to

$f$ In this exprestion, $h_{\rho}$ is the $\gamma \pi \rho$ coupling constant, $\vec{x}$ is the pion feld, where the arow refers to the three isospin components, $\vec{p}$ is the $\rho$ field, and $F_{a p}$ is the electromagnetic field strength tensor, which is composed of the photon field. 


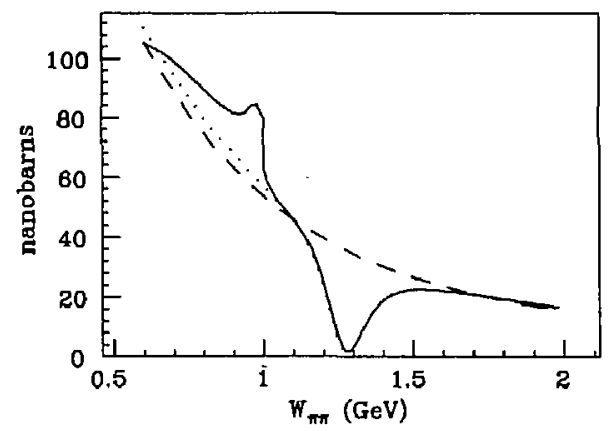

Figure 7.6. The Mennessier model at $\theta=\pi / 2$ with and without unitarity corrections for final state interactions. The dashed line shows the simple Born term, which is identical to Eqn. 7.27, the dotted line includes unitarization of the $D$-wave, and the solid line includes unitarization of the $S$-wave us well. No direct resonance couplings are included.

see the effect of unitarization on the Born term. Figure 7.6 shows the invariantmass spectrum at $\theta=\pi / 2$ with no terms in the Lagrangian for coupling of $\gamma \gamma$ to s-channel resonances. We find that even with no resonance coupling, the $S^{*}(980)$ scalar resonance forms a quite prominent peak. The effect of the $J=2, I=0$ phase shift in the $f$ region actually is a dip rather than a peak. This is similar to the result obtained in Section 7.4.4. From equations 7.45 and 7.47, one can see that the additional term, involving the imaginary part of $g$, which was added to the $J=2, I=0$ part of the amplitude in order to satisfy unitarity, is negative at the resonance peak with respect to the Born term. In fact, it precisely cancels the $J=2, I=0$ part of the Born term at $s=m_{f}^{2}$.

Finally, there are direct s-channel resonance contributions, for both scalar and tensor mesons, which have $\gamma \gamma$ couplings to be adjusted at will. These couplings are the only parameters in the model which are not determined by strong interaction data, so they may be measured by fitting the model to data.

\subsection{COMPARISON OF THE TWO MODELS}

In this section we look at the shapes of the invariant-mass and angular distributions of the models for $\gamma \rightarrow \pi^{+} \pi^{-}$presented in the previous two sections, which we refer to as the Lyth model and the Mennessier model. When fitting these distributions to the data in Chapter 8 , it is necessary to convolute them with the two-photon luminosity function and to integrate over the detector acceptance. Here we simply present the $\gamma \gamma \rightarrow \pi^{+} \pi^{-}$predictions for the invariant mass spectrum at a fixed angle of $\theta=\pi / 2$, which is the center of the DELCO acceptance.

First, consider the Born term, which is, before unitarity corrections, exactly the same in both models. As must be the case for two photons annihilating to form two particles of spin zero, the helicity-two cross section goes to zero at $\cos \theta= \pm 1$. However, as the energy increases, the angular distribution becomes more and more flat, and the points where it begins to fall to zero move closer to $\cos \theta= \pm 1$. In contrast, the helicity-zero amplitude is sharply peaked toward small angles. However, it falls rapidly with increasing energy, compared with the helicity-two amplitude, and is not very significant within the DELCO acceptance, is one can Bee from Fig. 7.7. Thus the Born term is, within the DELCO acceptance, rather well approximated by a $1 / \mathrm{s}$ energy dependence and a uniform angular distribution.

The $f(1270)$ resonance contributions are not identical for the two models. However, they do have the same angular distributions, since a single resonance is produced only in a single partial wave. Both models allow the introduction of a resonance contribution to the $J=2$ partial wave in both the helicity-zero and helicity-two amplitudes, for which the angular distributions are plotted in Fig. 1.2. In such a case, the twa-photon width of the $f$ must be described by two independent parameters, $\Gamma_{\gamma \gamma}^{\lambda}\{\lambda=0,2\}$, such that $\Gamma_{\gamma \gamma}=\Gamma_{\gamma \gamma}^{0}+\Gamma_{\gamma \gamma}^{2}$. The energy dependence of the resonance terms, however, differs between the two models. To see tirat, let us compare then for the most simple case, where unitarity corrections are neglected. 


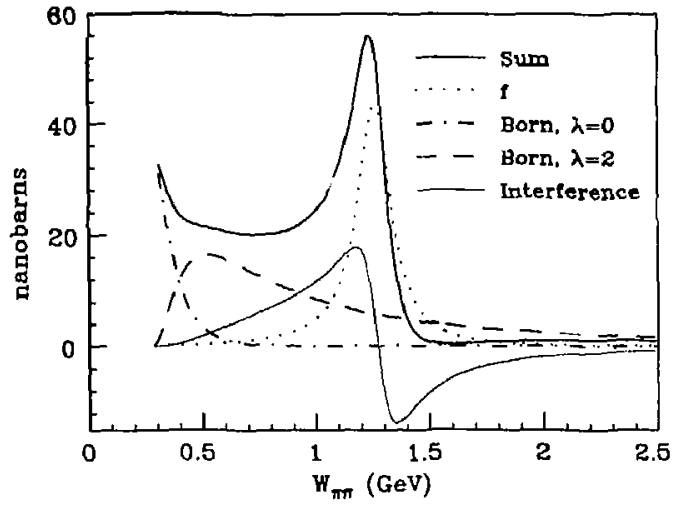

Figure 7.7. Prediction for the $\gamma \gamma \rightarrow \pi^{+} \pi^{-}=\pi$ an section at $\theta=\pi / 2$ asauming a simple model of the Born term. plus the helicity-two BreitWigner amplitude, with interference of the helicity-two amplitudes. $\Gamma_{\gamma 7}=3.0 \mathrm{keV}$ is assumed, and the partial waves are not sequired to satisfy the unitarity condition.

The energy dependence of $I(s)$ in the Lyth model is determined by Eqn. 7.32. Note that it affects both the phase and the magnitude of the resonance amplitude. The phase is consistent with the measurements shown in Fig. 7.4, which is the same data as that which Mennessier used to fit his model. However, the energy dependence if $\Gamma_{7 y}$ in the numerator of the Breit-Wigner amplitude, Eqn. 7.34 need not be the same as that of $\Gamma(s)$, which appears in the denominator and in the phase. Changing the energy dependence shown in Eqn. 7.32 , by changing the parameter $a$, for example, actually produces only minor changes to the resonance shape itself. That is because although the taits are strongly affected, they already are small. With the interference shown in Fig 7.7, however, the lower tail of the resonance plays an important part, and its energy dependence becomes significant. As a result, it is difficult to infer from the data whether any additional contributions, from broad scalar resonances, for example, are necessary in the region between about $0.5 \mathrm{GeV}$ and $1.0 \mathrm{GeV}$, unless one is very confident with the parameterization of the $f$. Fortunately, the fitted value of the two-photon width itself remains insensitive to the detailed parameterization of the energy dependence.

Figure 7.8 shows a comparison of the invariant-mass distributions of the two models. Below the $f$ the Mennessier madel gives a significantly lower cross section than the other, even though the two use exactly the same formula for the Born term (no unitarization corrections are included in either). When an additional energy dependence, or form factor, defined by

$$
\Gamma_{77} \Rightarrow \Gamma_{7 \gamma} \cdot \frac{a+m_{f}^{2}}{a+s},
$$

is introduced into the resonance contribution of the Mennessier model, 60 then the two models agree quite well for $a=1.25 \mathrm{GeV}^{2}$. It turns out that the Mennessier model the data on the low side of the resonance peak very poorly without this modification. Therefore, all subsequent calculations made with the Mennessier model assume the energy dependent width given by Eqn, 7.58 .

Now let us consider the effects of requiring the models to satisfy the unitarity condition. Here is where some troublesome differences between the two appear. From Fig. 7.9, one sees that in the resonance region both models suffer a decrease in the cross section at the $f$ peak when unitarity is required, but the decrease is much more severe for the Lyth model. We do not understand why that is so, $f$ since one would expect about the same result from each. In the Lyth model we have required elastic unitarity of the amplitude. Mennessier includes in addition the effects of the coupled $K K$ channel, but the branching ratio of the $f$ to $K K$ is only $3 \%$, and the total inelasticity is but $16 \%$, so it is hard to imagine how that rould make much difference. Furthermorc, the effect of unitarization when there

- The Mennessier model is essentially a black box to us, in the form of 700 lines of FORTRAN code. Only the detaile of the Lyth model can be given here. 


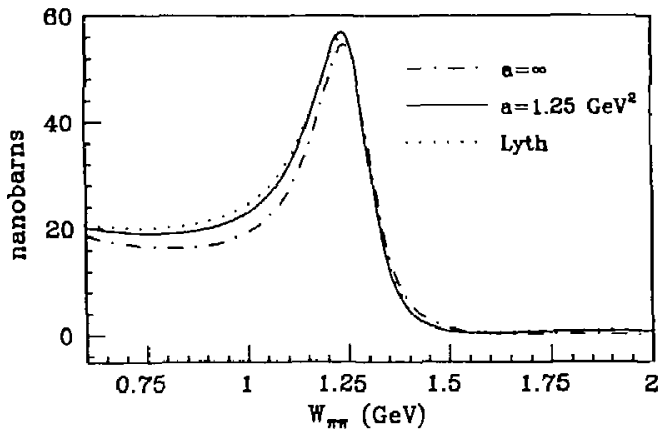

Figure 7.8. Effect of the energy dependence of the $f$ resonance on the mass distribution of the Mennessier model at $\theta=\pi / 2$. The parameter $a$ determines the strength of the form factor, which goes at zero as $a \rightarrow \infty$. Also shown for reference is the same calculation using the Lyth model.

is no direct coupling of $\gamma \gamma$ to the $f$, as show. in Fig. 7.6, does not seem to be consistent with what we see in Fig. 7.9 with $\Gamma_{\eta \gamma}=3.0 \mathrm{keV}$. This question has not been resolved, but there is yet another difference to consider.

The two curyes shown in Fig. 7.9 of the Lyth model differ from each other only by the addition of an imaginary part, according to Eqn. 7.47, to the unchanged real part of the coupling $g$. The real part of $g$ thus remains $\Re_{g}=m_{f}\left[\Gamma_{\gamma \gamma} \Gamma_{\pi \pi}\right]^{1 / 2}$, with $\Gamma_{\gamma \tau}=3.0 \mathrm{keV}$. But Lyth defines ${ }^{53}$ the two-photon width by $|g|=m_{f}\left[\Gamma_{\gamma \gamma}^{\text {lyth }} \Gamma_{\pi \pi}\right]^{1 / 2}$. Thus he considers the unitarity correction to be part of the coupling of the resonarce to the two photons.

Mennessier's definition is different. The unitarity correction is considered to be part of the Born term, yielding the unitarized Born term, to which the "direct coupiing" of the resonance to $\gamma \gamma$ is added. Thus in Fig. 7.6, we see an obvious $S^{*}$ peak and a big effect from the $f$ even though all direct couplings of resonances were se: to zero. Also, consider Ref. 61, in which the $f$ signal observed with the

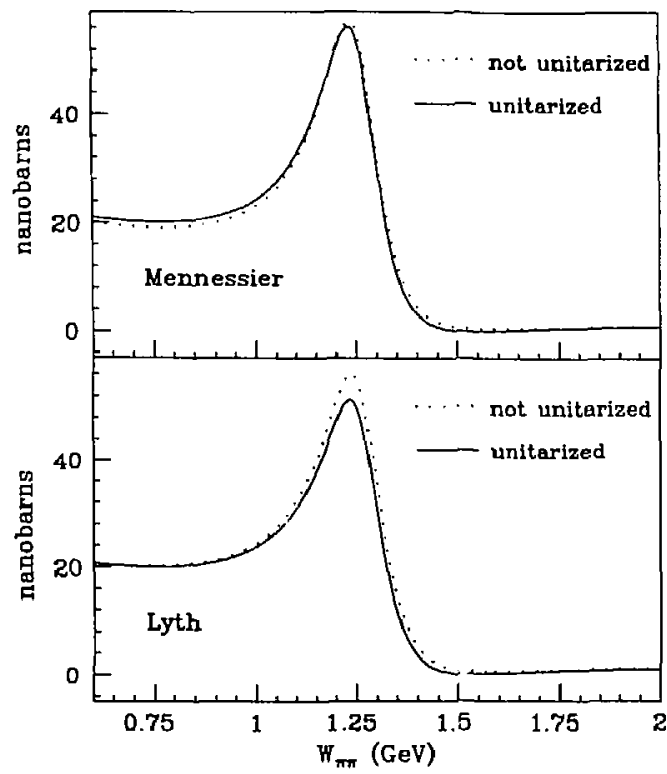

Figure 7.9. The effect of requiring unitarity for the $D$-wave on both the Lyth and Mennessicr models.

CELLO detector is 6t by the model of Mennessier. The authors state,

"... the only free parameter fitted to our data is $\Gamma_{7 \gamma}\left(f_{0}\right)$ which describes the 'direct' $\gamma \gamma j_{0}$ coupling. This does not correspond exactly with the observed $f_{0}$ signal since the helicity 2 projection of the Born term may give a small contribution to the $f_{0}$ signal by final state scattering effects... $F_{\gamma \gamma}\left(f_{0}\right)$ is the relevant varameter for comparison with theoretical predictions based on internal meson structure (e.g., the quark model), whereas in evaluating dispersion reiations or sum rules the full partial wave amplitudes measured by the fit to the experimental distributions should be used." 
It is true that in the quark model, the coupling of photons to a meson must be real, but then the quark model also has no provision for describing the finite width and associated energy dependencics of a resonance. Recall that if the resonance width may be neglected, then the imaginary part of the coupling, as specified in Eqn. 7.47, may also be neglected. Thus it is clear that comparisons with quark model predictions, such as the $S U(3)$ predictions for the ratios of two photon widths, always will be limited in validity by the simple fact that in reality the tensor mesons have relatively large widths, compared with the pseudoscalar mesons, for example.

Perhaps a more important question about the definition of $\Gamma_{\gamma \gamma}$ is how to relate the charged and neutral pion-pair channels. The non-resonant part of the neutral cross section is small enough that whether the reson ant coupling is complex becomes irrelevant - there simply are no significant interference effects. One would like to define $\Gamma_{\gamma \gamma}$ such that, for both the charged and neutral cases, the resonant contribution to the cross section is proportional to it. In other wordo, the arme quantity should be measured whether one studies $\pi^{+} \pi^{-}$data or $\pi^{0} \pi^{0}$ data.

Recall that the prediction for $\$ g$ was obtained from considering the sum of the $f$ resonance and the $I=0, J=2$ projection of the Born term and requiring it to satisfy the unitarity condition. The Born term contains contributions to both $I=0$ and $I=2$, while the resonance is only $I=0$. The unitarization procedure adds a contribution to the $I=0$ amplitude, but the $I=2$ amplitude is unchanged. The observed cross section then is calculated by taking the sum of the $I=0$ amplitude and the unitarized $I=2$ amplitude, projecting it onto either $\left\langle\pi^{+} \pi^{-}\right\rangle$or $\left(\pi^{0} \pi^{0}\right\}$, according to Eqn. 7.19, and squaring the absolute magnitude. The Born term, of course, vanishes for the neutral final state, but the contribution of the term required to unitarize the $I=0$ amplitude remains for both the charged and neutral final states. Therefore, in order for the model of Lyth to be consistent, his definition of the two-photon width must be accepted when using his model. A simple physical picture of how the Born term can affect $\gamma \gamma \rightarrow \pi^{0} \pi^{0}$ in the $f$ region is to imagine charged pion pairs being formed from the Born term and then resonating in the final state to $\pi^{0} \pi^{0}$.

It is not clear exactly how to interpret Mennessier's model. In particular, it is difficult to understand what is the physical significance of the distinction between "direct coupling" of $\gamma \gamma$ to the $f$ and the contribution coming from unitarization. After all, the strong interactions occur on such a short time scale relative to the electromagnetic interactions that they cannot be considered in any meaningful sense to have occurred after and separate from the $\gamma \gamma$ coupling. In any case, in Chapter 8 we give results from data for toth definitions and also for the case where no unitarization corrections at ail are made. We will see that in fact the experimental errors are small enough for our $\pi^{+} \pi^{-}$measurement that these problems cannot be neglected. Unfortunately, the statistical errors on measurements of the corresponding neutral channel are not yet small enough to check for consistency.

As a final note, we should point out that the $\int$ peak observed in $\pi^{0} \pi^{0}$ by the Crystal Ball experiment is about $40 \mathrm{MeV}$, or $3 \%$, low compared with the known value of $1274 \mathrm{MeV}$. That is about the same magnitude of downward shift as observed in the charged channel. However, it is not clear whether it is real, bec ause the systematic uncertainty in the energy acale is as large as $2 \%$, and the statistical error on the determination of the peak position is $1.1 \%$. $\dagger$ Such a downward shift in the neutral channel is not explained by either model so far considered. Presumably the neutral channel must have some continuum production from such processes as $\omega$ exchange, but its level cannot be determined from the available data-certainly there is no obvious large continuum as in the charged channel. Mennessier includes vector meson exchanges in his model and predicts that the $\pi^{0} \pi^{0}$ peak is at least $30 \mathrm{MeV}$ above the $\pi^{+} \pi^{-}$peak.t

i The ft of the resonance width in the Crystal Ball data yields $\Gamma_{f}=248 \pm 38 \mathrm{MeV}$, which is atmost two standard deviations high and also is unexplained.

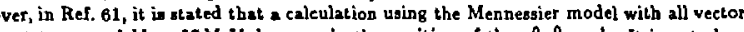
exchanges set to sero yields a $20 \mathrm{MeV}$ decrease in the position of the $\pi^{0} x^{0}$ peak, It is not clear 
In a recent paper ${ }^{62}$ a model has been proposed to explain why the $\pi^{+} \pi^{-}$and $\pi^{0} \pi^{0}$ peaks might be identical. The model assumes some sort of $I=2$ contribution in the $f$ region, from a non-resonant process or an exotic resonance, in addition to the large $I=2$ component of the $\gamma \gamma \rightarrow \pi^{+} \pi^{-}$Born term. This additional $I=2$ component effectively cancels that of the Born term in the $f$ region. Then the amplitude in the $f$ region is purely $I=0$, in which case the $\pi^{0} \pi^{0}$ cross section must be the same as that for $\pi^{+} \pi^{-}$in shape and half as large.

Nonetheless, we shall continue to assume that the Born term describes the continuum for the charged channcl. The deviation of the peak position in the neutral channel could very well be an experimental effect.

whether that is consistent with Mennessier's conclusions.

\section{Fitting the $\pi^{+} \pi-$ Spectra}

8.1 SUMMARY OF THE $\gamma \gamma \rightarrow \pi^{+} \pi-$ MODEL

The $\pi^{+} \pi^{-}$data is to be fit to the phenomenological model of Chapter 7 , consisting of the Born term calculated from exchange of point pions (Eqn. 7.27) plus terms for the $f$ resonance calculated according to Eqn. 7.34 with the energy dependent width of Eqn. 7.32. The resonance can be formed in both helicity-zero and helicity-two states, so there are two parameters to deseribe the coupling to two photons: $\Gamma_{\gamma \gamma}^{0}$ and $\Gamma_{7 \gamma}^{2}$, where $\Gamma_{\gamma \gamma}=\Gamma_{\gamma \gamma}^{0}+\Gamma_{7 \gamma}^{2}$. The resonant partial wave amplitudes of Eqn. 7.34 must be multiplied by the appropriate angular factors, as given in Eqn. 7.22, in order to obtain the full amplitudes. Furthermore, the amplitudes are forced to satisfy the unitarity condition of Eqn. 7.43 by the addition of an imaginary part to the resonant coupling, according to Egn. 7.47. However, no unitarity correction is made for the helicity-zero amplitude, because the $J=2$ part of the helicity-zero Born term is negligible in the $f$ region.

In summary, the cross section is given by

$$
\begin{aligned}
& \frac{\mathrm{d} \sigma}{\mathrm{d} \Omega}\left(\gamma \gamma \rightarrow \pi^{+} \pi^{-}\right)=\frac{1}{2}\left|B_{0}(s, t)+5 \sqrt{\frac{2}{3}} d_{00}^{2}(\theta) R_{20}^{0}(s)\right|^{2} \\
& +\frac{1}{2}\left|B_{2}(s, t)+5 \sqrt{\frac{2}{3}} d_{20}^{2}(\theta) R_{22}^{0}(s)\right|^{2}, \\
& \text { where } d_{00}^{2}(\theta)=\frac{3}{2} \cos ^{2} \theta-\frac{1}{2} \text {, } \\
& d_{20}^{2}(\theta)=\frac{\sqrt{6}}{1} \sin ^{2} \theta \text {, } \\
& \text { and } R_{2 \lambda}^{0}(s)=\frac{2}{\sqrt{a}} T_{2 \lambda}^{0}(s)=\frac{2}{\sqrt{s}} \frac{g_{\lambda}(s)}{m_{f}^{2}-s-i m_{f} \Gamma(s)} \text {, } \\
& g_{0}(s)=m_{f}\left[\left.\Gamma_{0}(s) \Gamma_{\pi \pi}(s)\right|^{1 / 2}\right. \text {, } \\
& g_{2}(s)=m_{f}\left|\Gamma_{2}(s) \Gamma_{\pi \pi}(s)\right|^{1 / 2}+i \sqrt{\frac{2}{3}} m_{f} \Gamma(s) B_{22}\left(m_{f}^{2}\right) \text {. }
\end{aligned}
$$

Recall that $\Gamma_{\pi \pi}(s)=0.843 \cdot \Gamma(s)$ and that the $s$ dependence of $\Gamma(s)$ is given by Eqn. 7.32. The $s$ dependence of $\Gamma_{0}(s)$ and $\Gamma_{2}(s)$ also is given by Eqn. 7.32, but with $m_{\pi}$ replaced by $m_{\gamma}=0$. This cross section is multiplied by the appropriate 
$\gamma \gamma$ luminosity function to give the prediction for $e^{+} e^{-} \rightarrow e^{+} e^{-} \pi^{+} \pi^{-}$, and finally, the two-photon widths to be fitted are defined by the expressions

$$
\begin{aligned}
& \left|g_{0}\right|^{2}=m_{f}^{2} \Gamma_{\gamma \gamma}^{0} \Gamma_{\pi \pi}\left(m_{f}^{2}\right) \\
& \left|g_{2}\right|^{2}=m_{f}^{2} \Gamma_{\gamma \gamma}^{2} \Gamma_{\pi \pi}\left(m_{f}^{2}\right) .
\end{aligned}
$$

Thus the complete two-photon width may be written as

$$
\begin{aligned}
\Gamma_{\tau \gamma} & =\Gamma_{\tau \gamma}^{0}+\Gamma_{\gamma \gamma}^{2} \\
& =\Gamma_{0}\left(m_{f}^{2}\right)+\Gamma_{2}\left(m_{f}^{2}\right)+\frac{\left[3 g_{2}\left(m_{f}^{2}\right)\right]^{2}}{m_{f}^{2} \Gamma_{\pi \pi\left(m_{f}^{2}\right)}} \\
& =\Gamma_{0}\left(m_{f}^{2}\right)+\Gamma_{2}\left(m_{f}^{2}\right)+0.257 \mathrm{keV},
\end{aligned}
$$

This corresponds to the definition by $\dot{L}_{y}$ th. The definition of $\Gamma_{77}$ used by Mennessier is roughly the same as that of Eqn. 8.3 without the additional $0.257 \mathrm{keV}$ added (see Section 7,6).

\subsection{THE FutTing METHOD}

The fit itself may be done in two ways. E:ther the true $\pi^{+} \pi^{-}$spectra are unfolded from the data and then compared directly with the calculation, or else the detector effects are folded into the Monte Carlo calculation, giving Monte Carlo spectra to be compared directly with the data. We take the latter approach for reasons of convenience. The Monte Carlo prediction for the pion spectrum is added to the QED prediction for the muon spectrum plus the estimates of the other minor backgrounds. That sum is compared to the uncorrected data. The precise normalizations of the backgrounds are allowed to vary within experimental errors and hence depend on the results of the fit.

There are several parameters which are adjusted simultaneously in the fit besides the two-photon widths. However, that does not mean that there is a lot of freedom available to fit the model to the data, because all parameters except for the two-photon widths are considered to be known within certain error limits and are constrained to remain within those limits. That is done by adding a penalty function to the $x^{2}+\dagger$ For $n$ bins in the data and Monte Carlo and $m$ constrained parameters, we have

$$
x^{2}=\sum_{i=1}^{n} \frac{\left(y_{i}-y_{i}^{\mathrm{mc}}\right)^{2}}{\sigma_{i}^{2}}+\sum_{j=1}^{m} \frac{\left(p_{j}-\bar{p}_{j}\right)^{2}}{\sigma_{p_{j}}^{2}}
$$

The $y_{i}$ are the bin contents, and the $p_{j}$ are the adjustable parameters, $\bar{p}_{j}$ their nominal values, and $\sigma_{p_{j}}$ their uncertainties. In addition, we will consider some fits in which the Born terms and the interference terms are multiplied by factors (most appropriately termed fudge factors) which are allowed to vary freely in the fit in order to judge the sensitivity to some assumptions made in the model.

In cases where an assumption is made to constrain to a fixed value the ratio $\Gamma_{\gamma \gamma}^{0} / \Gamma_{\gamma \gamma}^{2}$, only the invariant-mass distribution is included in $\chi^{2}$. When the ratio is allowed to vary in the fit, then it is necessary to include in $\chi^{2}$ the distribution of $\cos \theta_{\mathrm{cm}}$ as well.

The complete list of parameters which may be adjusted simultaneously in the fit is

1. The $\gamma \gamma$ width of the $f$. $\Gamma_{\gamma \gamma}$

2. Ratio of widths. $\quad \Gamma_{\gamma \gamma}^{0} / \Gamma_{7 \tau}^{2}$

3. Helicity-one contribution. $\quad \Gamma_{\gamma \gamma}^{1} / \Gamma_{\tau \gamma}$

4. The unitarity correction. $\quad \exists g_{2}\left(m_{f}^{2}\right)=(2.5 \pm 0.7) \cdot 10^{-4} \mathrm{GeV}^{2}$

5. The $f$ mass.

$m_{f}=1.274 \pm 0.013 \mathrm{GeV}$

6. Full width of the $f$. $\quad r_{f}=0.178 \pm 0.020 \mathrm{GeV}$

7. Effective luminosity. $\quad \mathcal{L}_{\text {eff }}=102.3 \pm 2.3 \mathrm{pb}^{-1}$

8. The $\eta^{\prime}$ background. $\quad N_{\eta^{\prime}}=468 \pm 87$ events

9. The $K^{+} K^{-}$and p p background. $\quad N_{\mathrm{tol}}=342 \pm 34$ events

$f$ Doing the fit by minimising $x^{2}$ assumes gaussian, rather than Poisson, distributions for the contents of each bin. In this case that is a very good approximation, because the number of eventa is greater than 200 for every bin of the antagged data. 
10. The remaining background.

11. $\pi^{+} \pi^{--}$efficiency error at $0.6 \mathrm{GeV}$.

$N_{\text {had }}=150 \pm 50$ events

$\varepsilon_{1}^{\pi}=0 \pm 0.10$

$\varepsilon_{2}^{\pi}=0 \pm 0.05$

12. $\pi^{+} \pi^{-}$efficiency error at $2.0 \mathrm{GeV}$

$\alpha_{B}=1$

13. Fudge factor for the Born term.

$a_{I}=1$

The values given here are appropriate for the $u$ tagged ar. alysis, although nonzero $\Gamma_{\gamma \gamma}^{1}$ is considered only in the tagged analysis. The error on the $f$ mase has been increased over the published value to account for uncertainty in the energy scale of the experiment. Parameter number ten refers to the hadronic background estimated from the number of pion-pair events found with non-zero charge. It and parameters eight and nine refer only to background normalizations; the shapes of the backgrounde are fixed. Note that thase backgrounds are almost insignificant, especially in the $f$ region, so the normalization parameters introduce little additional freedom into the fit. Notmally $\alpha_{B}$ and $\alpha_{I}$ are fixed at unity.

Parameters 11 and 12 deserve some further discussion. They refer to Eqn. 6.3, which gi-es the uncertainty limits on the measurement of the detection efficiency as a function of the invariant mass. The fit allows the normalization of the $\pi^{+} \pi^{-}$ Monte Carlo to vary within the limits given by Eqn. 6.3, but the individual bins are not allowed to vary independentiy. Only the normalization corrections at the upper and lower $W$ limits may vary independently - the corrections for each of the bins in between are determined by those at the upper and lower limits by Eqn. 6.3, which represents a smooth parabola with zero slope at the upper limit.

Since the theoretical model must be calculated by a large number of Monte Carlo iterations, then it is not completely straightforward to vary simultaneously all parameters for the purpose of doing the fit. But a method has been found which works well and is efficient. It relies upon the use of weighted evenis in the Monte Carlo. When generating events, the dominant $1 / W^{3}$ behavior of the cross section is produced by an analytic change of variables (Bee Appendix $B$ for a discussion of importance saropling in Monte Caslo integration), and all remaining factora are lumped together to produce an event weight. Instead of using a rejection algorithm to produce unweighted events, one may simply accumulate histograms of weighted cvents. Such an approach is the more efficient one, unless the detector simulation is very long and there is a large fraction of events with very small weights.

The method also relies on the fact that, for all but two of the parameters, the $\pi^{+} \pi^{-}$prediction plus background may be expanded into a sum of terms such that the parameters appear as factors multiplying yarious terms. That obviously is the case for the normalization corrections of the various backgrounds. Also, the correction to $\mathcal{L}_{\mathrm{eff}}$ is a factor multiplying hoth the $\mu^{+} \mu^{-}$and $\pi^{+} \pi^{-}$predictions, while the pion efficiency correction multiplies only the $\pi^{+} \pi^{-}$prediction. The mass and width of the $f$ are exceptions and are handled by a special technique. For the other parameters, we define two more factors by $\Gamma_{0} \Rightarrow \alpha_{0} \Gamma_{0}$ and $\Gamma_{2} \Rightarrow \alpha_{2} \Gamma_{2}$, so the cross section in Eqn. 8.1 may be written as

$$
\begin{aligned}
\frac{\mathrm{d} \sigma}{\mathrm{d} \Omega} & =\alpha_{B} \frac{\mathrm{d} \sigma_{B}}{\mathrm{~d} \Omega}+\frac{1}{2} \alpha_{0} g_{0}^{2} R_{0}^{2}+\sqrt{\alpha_{0}} \alpha_{B} \alpha_{J} g_{0} B_{0} R_{0} \cos \delta \\
& +\frac{1}{2} \alpha_{2}\left(\Re g_{2}\right)^{2} R_{2}^{2}+\sqrt{\alpha_{2}} \alpha_{B} \alpha_{J}\left(\Re_{g_{2}}\right) B_{2} R_{2} \cos \delta \\
& +\frac{1}{2}\left(\Im g_{2}\right)^{2} R_{2}^{2}-\alpha_{B} \alpha_{J}\left(\Im_{g_{2}}\right) B_{2} R_{2} \sin \delta, \\
\text { where } \quad R_{\lambda} & \equiv 5 \sqrt{\frac{2}{3}} d_{\lambda{ }_{0}}^{2}(\theta) \frac{2}{\sqrt{s}}\left[\left(m_{f}^{2}-s\right)^{2}+m_{j}^{2} \Gamma(s)^{2}\right]^{-1 / 2}, \\
\frac{\mathrm{d} \sigma_{B}}{\mathrm{~d} \Omega} & =\frac{1}{2}\left(B_{0}^{2}+B_{2}^{2}\right), \\
\tan \delta & =\frac{m_{j} \Gamma(s)}{m_{f}^{2}-s} .
\end{aligned}
$$

In the Monte Carlo calculation, a weight is calculated for each of the seven terms, assuming some nominal values for the two radiative widths. Furthermore, for all but the first term, a different weight is calculated for each of twenty-five combinations of values for the $f$ mass and width. After the detector simulation, weighted histograms of both the $W$ and $\cos \theta_{\mathrm{cms}}$ distributions are produced for each of the total of 151 weights. To allow for the fitting program to have access to values of $m_{f}$ and $\Gamma_{f}$ between those included in the $5 \times 5$ arrays, each array, 
one for each bin of each of the six terms, is interpolated by bicubic splines before beginning the iterations of the fit. Thus the fitting program is able quickly to add up the eross section uefined by Eqn. 8.5 for any set of values of the 13 parameters (except that the values of $m_{f}$ and $\Gamma_{f}$ are limited to the range of the spline fits). Since all of the weights are calculated in a single Monte Carlo job, and every weight in a single event is calculated for the same $W$ and $\cos \theta_{\mathrm{cms}}$, then there are no statistical fluctuations of one term of Eqn. 8.5 relative to another. Therefore, the statistical error of the sum of terms is just a linear sum of the statistical errors of the individual terms (the resulting error is the same as if all terms had been added together in the Monte Carlo job and accumulated into a single histogram). Finaliy, the general fitting program MINUIT ${ }^{83}$ makes the job of fitting the Monte Carlo histograms to the data eagy, convenient, and reliable.

\subsection{Fit RESUlts fOR THE UNTAgGED ANALYSIS}

In this section we compare the results for the fit of the $f$ two-photon width under various theoretical assumptions. First, let us consider the most simple model, in which no unitarization corrections are made and the $f$ is assumed to be produced only with helicity-two.

\subsubsection{Fit to the $\pi^{+} \pi^{-}$Model Without Unitarization}

The theoretical model is almost identical to that used in the 1984 publication of DELCO results on $\gamma \gamma \rightarrow \pi^{+} \pi^{-} .64$ There are only three differences: First, the parameterization of the energy dependent width is different. In Ref. 64 a form due to Blatt and Weisskop $f^{65}$ is used, which gives a result for the phase shift almost identical to that obtained from Eqn. 7.32 with $a=1 \mathrm{GeV}^{2}$. However, using $a=0.5 \mathrm{GeV}^{2}$ gives a better fit to the data shown in Fig. 7.4. The second difference is that Ref. 64 assumes an energy dependence for $\Gamma_{\tau \gamma}$ according to $\Gamma_{\gamma \gamma}(t)=\left(\sqrt{s} / m_{\jmath}\right) \Gamma_{\gamma \gamma}$, whereas the form used here is Eqn. 7.32 for both $\Gamma_{\gamma \gamma}$ and $\Gamma_{\pi \pi}$. Finally, Ref. 64 uses a value for the branching ratio of the $f$ to $\pi \pi$ which is
$1.5 \%$ lower than the value now found in Ret. 6. These differences all are minor, and one finds that the two predictions can only barely be distinguished from each other on a plot like that found in Fig. 7.7.

The invariant-mass spectrum to which the Monte Carlo predictions are fit is shown in Fig. 5.7a. When only the two-photon width is allowed to vary in the fit and only the points in the range from $0.85 \mathrm{GeV}$ to $1.40 \mathrm{GeV}$ are included in the fit, the result is $\Gamma_{\Upsilon 7}=2.68 \pm 0.07 \mathrm{keV}$. The $\chi^{2}$ for the nine bins of data included over the range of the $f$ peak is 6.7. Below the $f$ peak the Monte Carlo prediction is on average about $7 \%$ higher than the data, but that is within the uncertainty limits on the trigger efficiency in that range. From this we conclude that the model gives a reasonable fit to the data even with the efficiencies and effectiv + luminowity fixed exactly at the messured values. Also, the utatistical error on the two-photon width coming only from Buctuations in the bins of the data and ivion tic Carlo is $0.067 \mathrm{keV}$, or $2.5 \%$. However, we know that the efficiencies and effective lumirosity, and even the $f$ mass and full width, can be varied considerably about the mei sured values and still be within experimental errors. This reduces the pridictive power of the model, allowing it to fit the data more closely and resuiting in aystematic orror on the two-photon width which are much larger than the atatistical error.

Table 8.1 shows in detail the results of allowing all of the experimental parameters to vary within their known error limits. The region below the $f$ is included in the fit, and as usual, the two-photon width is allowed to vary freely. All of the parameters remain within their error limits, including the $f$ mass and full width. Note that the fitted error estimates for $m_{j}$ and $\Sigma$, actually are smaller than the constraints imposed on them in the fit. In fact, if $m_{f}$ and $\Gamma_{f}$ are allowed to vary completely freely in the fit, they still remain within their error limits. However, the data in the low range of $W$ prefer that the $\pi^{+} \pi^{-}$detection efficiency be almost $5 \%$ lower at $W=0.6 \mathrm{GeV}$ than it was messured to be, and the effective luminosity is decreased by $1.5 \%$ in order to predict fower muon pairs and pion pairs in that region. Furthermore, by shifting the $f$ mass down slightly, the Monte Carlo 
prediction fits in the peak of the $f$ better. All of these changes are well within the respective error limits, but they together have the effect of increasing the fitted two-photon width by $4 \%$ over the value obtained when all other parameters are fixed. Nonetheless, this shift in $\Gamma_{T r}$, though greater than the statistical error, is well within the total error shown in Table 8.1 of $\pm 7 \%$.

If only those bins with $0.95 \leq W \leq 1.40$ are included in the fit, then the result is $\Gamma_{\gamma 7}=2.71_{-0.25}^{+0.26} \mathrm{keV}$, which is almost the same as the result obtained when all parameters but $\Gamma_{T \gamma}$ were held fixed, although here the systematic errors are included. Another systematic error which has not yet been included is the uncertainty in the momentum resolution of the experiment (see Section 3.1). The fits presented so far have assumed that the resolution of the track curvature is $\sigma_{\kappa} / \kappa=7 \%$. When it is varied from $8 \%$ down to $6 \%$, the two-photon width from the fit varies by less that the statistical precision of $2.5 \%$, while the fitted value of the full width varies from $0.164 \mathrm{GeV}$ up to $0.187 \mathrm{GeV}$. Thus the result is not sensitive to the value assumed for the momentum resolution, and the observed $f$ peak is completely consistent with the known value of the $f$ width.

$A$ check is made on the validity of using the Born term to describe the continuum below and under the $f$ by doing a fit with the coefficient $\alpha_{B}$ left free to vary. The range in $W$ from $0.6 \mathrm{GeV}$ to $1.4 \mathrm{GeV}$ is included in the fit, and the main effect is that $\alpha_{B}$ decreases by $7 \%$ from unity, while the $\pi^{+} \pi^{-}$trigger efficiency returns to its measured value. The value for the two-photon width increases by only $1.4 \%$, while the size of the error estimate increases by $0.01 \mathrm{keV}$. Therefore, within experimental errors the Born term describes the continuum adequately at least below the $f$ peak, and the filied two-photon width is not changed when the normalization of the Born term is allowed to vary freely.

Figure 8.1 shows a histogram of the data with all of the background subtracted. It is compared with the $\pi^{+} \pi^{-}$prediction as given in Table 8.1. Note that the fit is excellent for $W \leq 1.40 \mathrm{GeV}$, but above that and up to $1.75 \mathrm{GeV}$ the data are much higher than the prediction and are only slightiy lower than
Table 8.1. Results of fitting the $\pi^{+} \pi^{-}$model with no unitarization and with the helicity-zero two-photon width of the $f$ fixed to zero. More complete definitions of the parameters may be found in Section 8.2.

\begin{tabular}{|c|c|c|c|c|c|c|c|c|}
\hline \multicolumn{4}{|c|}{ Parameter } & \multicolumn{2}{|c|}{ Fit Value } & \multirow{2}{*}{$\frac{\text { Lower Error }}{-0.19}$} & \multirow{2}{*}{\multicolumn{2}{|c|}{$\begin{array}{c}\text { Upper Error } \\
+0.20\end{array}$}} \\
\hline \multicolumn{4}{|c|}{$\gamma \gamma$ width } & 2.82 & keV & & & \\
\hline 5. & \multicolumn{3}{|c|}{$f$ mass } & 1.262 & $\mathrm{GeV}$ & -0.004 & \\
\hline 6. & \multicolumn{3}{|c|}{ full width } & 0.169 & $\mathrm{GeV}$ & -0.011 & \multicolumn{2}{|c|}{+0.011} \\
\hline 7. & \multicolumn{3}{|l|}{$\mathcal{L}_{\text {efI }}$} & 100.7 & $p^{b^{-i}}$ & -1.2 & \multicolumn{2}{|c|}{+1.2} \\
\hline 8. & \multicolumn{3}{|c|}{$\eta^{\prime}$ background } & 423 & events & -83 & \multicolumn{2}{|c|}{+83} \\
\hline 9. & \multicolumn{3}{|c|}{$K \bar{K}, p \bar{p}$ background } & 336 & events & -34 & \multicolumn{2}{|c|}{+34} \\
\hline 10. & \multicolumn{3}{|c|}{$Q \neq 0$ background } & 150 & events & -50 & \multicolumn{2}{|c|}{+50} \\
\hline 11. & \multicolumn{3}{|l|}{$\varepsilon_{1}^{\pi}$} & -0.047 & & -0.085 & & .086 \\
\hline 12. & $\varepsilon_{2}^{\pi}$ & & & -0.003 & & -0.050 & & .050 \\
\hline & & Covar & iance & Matrix Co & rrelation $\mathrm{C}$ & Coefficients & & \\
\hline & 1 & 5 & 6 & 7 & 8 & 9 & 10 & 11 \\
\hline 5 & -0.056 & & & & & & & \\
\hline 6 & 0.490 & 0.366 & & & & & & \\
\hline 7 & 0.011 & -0.248 & -0.0 & & & & & \\
\hline 8 & 0.063 & -0.064 & -0.0 & -0.037 & & & & \\
\hline 9 & -0.043 & -0.015 & -0.0 & 0.004 & -0.007 & & & \\
\hline 10 & -0.013 & -0.017 & -0.0 & -0.023 & -0.014 & -0.009 & & \\
\hline 11 & -0.324 & 0.297 & -0.2 & -0.803 & -0.101 & -0.002 & -0.019 & \\
\hline 12 & -0.729 & 0.104 & -0.0 & 0.021 & -0.016 & 0.001 & -0.004 & -0.023 \\
\hline $\begin{array}{l}\text { Fit } \\
x^{2}\end{array}$ & $\begin{array}{l}\text { nge: } \\
1.8\end{array}$ & $<W<$ & & & bins & & & \\
\hline
\end{tabular}




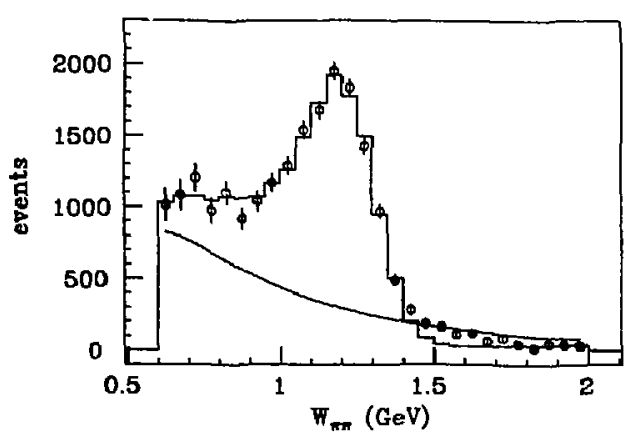

Figure 8.1. The background-subtracted $\pi^{+} \pi^{-}$invariant-mass spectrum compared with the Monte Carlo prediction (solid histogram) with no unitarization and with no helicity-zero coupling to the $f$. The smooth curve show the prediction for $\pi^{+} \pi^{-}$from the Born term alone. The values of all the fit parameters are as given in Table 8.1.

the spectrum predicted from the Borr. term alone. It is the interference with the resonance which makes the prediction so low in that region. Why the full effect is not seen in the data is not known.

If the entire range of $W$ is included in the fit, then as expecter, the effective luminasity is increased by almost $2 \%$ in order to reduce the data above the $f$ peak by subtracting more muon pairs. To compensate then below the $f$, the $\pi^{+} \pi^{-}$ detection efficiency is lowered to the point of being two standard deviations below ce mensured value. The net result is only a $2 \%$ increase in $\Gamma_{\gamma \gamma}$. The fit stiit is not gcord above the $f$ peak, with $\chi^{2}=48$ for the entire 28 bins of data. It is clear that this model cannot be used over the full energy range without some modification.

This disagreement above $W=1.4 \mathrm{GeV}$ is worrisome because it indicates that something is wrong with the description of the continuum background. It is important to assess what affect this has on the measurement of the two-photon width of the $f$. It is possible that sor.e of the effect is from non-gaussian tails in the momentum resolution which are not reproduced by the Monte Carlo simulation. However, the same effect is seen by the PEP*4/PEP-9 experiment5 Most of the disagreement is believed to be genuine.

In Ref. 5 the problem is handled by introducing an arbitrary parameter, which we call $\alpha_{\Gamma}$, to multiply the interference terms of ihe cross section. This does yield a good fit over the full energy range, but only with $\alpha_{I}$ as low as 0.5 . When $\alpha_{I}$ is allowed to vary freely in the DELCO fit, good agreement is achieved over the full energy range ( $x^{2}=26$ for the 28 bins), but with $\alpha_{I}=0.72$ for the best fit. However, the fit forces the $f$ mass down to $1.249 \mathrm{GeV}$, which is getting rather far from the preferred value. That is to be expected, since the interference is necessary to shift the peak down to the observed position. The best fit for $\Gamma_{\gamma \gamma}$ is raised by only $1.8 \%$ compared with the result for $\alpha_{I}=1$.

The parameter $\alpha_{I}$ is justified in Ref. 5 by arguing that one really does not know if the continuum is properly described by the Born term, in which case one cannot be sure that it really is all in helicity two in the $f$ region. However, multiplying the interference terma by a fudge factor is a perversion of the model which makes it difficult to interpret physically. Another possible explanation for the discrepancy is resonance production in the $S$-wave (perhaps the $c(1300)$ ). To assess the effect of such a possibility, we try adding an additional non-interfering bae'.rground to the fit. This is done with a gaussian curve centered at $W_{0}=1.5 \mathrm{GeV}$ with arbitrary width and height:

$$
B(W)=A \exp \left(-\frac{\left(W-W_{0}\right)^{2}}{2 \sigma^{2}}\right)
$$

The best fit over all $28 \mathrm{~W}$ bins has $x^{2}=15.0$ with $A=126$ events and $\sigma=0.13 \mathrm{GeV}$. I ne best fit for $\Gamma_{\gamma \gamma}$ is lower by $3.5 \%$ relative to the result fit only on the range $W<3.4 \mathrm{GeV}$ and without the gaussian background. Note that adding the gaussian background changes the fitted value of $\Gamma_{\gamma \gamma}$ in the opposite sense from the effect of the fudge factor in the interference term. 
Taking into consideration all of the systematic effects, and in particular the effects of changing the region included in the fit and adding the gaussian background of Eqn. 8.6, icade to the result

$$
\Gamma_{7 \gamma}=2.77 \pm 0.31 \mathrm{keV}, \quad(\lambda=2, \text { no unitarization }) .
$$

Keep in mind that this result assumes a model which violates unitarity and also that the helicity-zero coupling is assumed to be exactly zero. Since this is identical to the model used in the DELCO analysis of Ref. 64, we may compare the results directly. The result of the analysis of Ref. 64 is $\Gamma_{\gamma \gamma}=2.70 \pm 0.21 \mathrm{keV}$. Only about half of the presently available data were used, but the actual reasons for the difference with the present result are systematic. First of all, the analysis of Chapter 6 has been done since then. Reference 64 assumes that the relative detection efficiency of pion pairs compared with muon pairs is independent of energy. Second, in Ref. 64 the correction to $\mathcal{L}_{\text {eff }}$ necessitated by bremsstrahlung from electrons passing through the beampipe and drift chamber material was not made. These two corrections largely cancel each other with respect to the fitted two-photon width, but they do contribute significantly to the estimate of the systematic error.

\subsubsection{Effect of Requiring Unitarity}

The unitarity correction is added to the model by setting $\$ g_{2}$ nonzero and allowing it to vary in the fit. If it is allowed to vary completely frecly, then one finds that the data give no constraint. That is expected from Fig. 7.9, where it is evident that the correction does not change the shape of the spectrum significantly but only lowers the peak a bit. Therefore, $\$ g_{2}$ must be constrained to remain within the range of the theoretical prediction of Eiqn. 7.57. That is done by using the bounds given by Eqn. 7.57 as gaussian error limits. Assuming such a gaussian distribution cannot be well justified, but the bounds scem conservative enough that the error estimate is not likely to be an underestimate in any case.
We continue to assume that there is no helicity-zero resonance coupling. The result of the fit is in all respects almost identical to that done with no unitarization except that the two-photon width becomes larger and has slightly larger errors. The fitted value arrived at for $I^{2} \gamma \gamma$ when fitting over the range $0.6 \leq W \leq 1.4 \mathrm{GeV}$ with $\alpha_{B}=1$ is $\Gamma_{\gamma \gamma}=3.44_{-0.21}^{+0.23} \mathrm{keV}$. This is assuming the definition given by Lyth, as expressed in Eqn. 8.3. When all systematic effects, such as the dependence on the interval of $W$ included in the fit, are accounted for as in the provious section, the final result for the unitarized model of Lyth, assuming zero helicityzero coupling, is

$$
\Gamma_{\gamma \gamma}=3.34 \pm 0.35 \mathrm{keV}, \quad(\lambda=2, \text { unitarized }) .
$$

\subsubsection{Comparison with the Mennessier Model}

The Mennessier model may also be used to fit the two-photon width of the $f$. The procedure is similar to that discussed above, excepi that it in not possible to fit the $f$ mass and full width simultancously with the two-photon width in this case. It is not possible for us is separate the model into all of its separate terms as is done for the more simple model in Eqn. 8.5. Therefore, Mennessier's program was run once for each of several values of the two-photon width, and the result was interpolated by cubic splines.

Table 8.2 gives the results of the fit when all data up to $W=1.4 \mathrm{GeV}$ are included. The $x^{2}$ is not as low as that shown in the fit of Table 8.1 , but that is partly due to the inability to adjust the mass and full width of the $f$. Restricting the fit to only the region of the $f$ peak has the same effect as with the model of the previous aection. Taking that into account, pius the effects of changing $m_{f}$, $\Gamma_{f}$, and the Monte Carlo momentum resolution within the allowed limits gives the final result for the Mennessier model:

$$
\Gamma_{\tau \gamma}=2.93 \pm 0.30 \mathrm{keV}, \quad(\lambda=2, \text { Mennessier })
$$


Table 8.2- Results of fitting the Mennessier model to the untagged $\pi^{+} \pi^{-}$ data with the helicity-zero two-photon wiath of the $f$ fixed to zero. More complete definitions of the parameters may be found in Section $\mathbf{8 . 2}$.

\begin{tabular}{|c|c|c|c|c|c|c|c|}
\hline \multicolumn{3}{|c|}{ Parameter } & \multicolumn{2}{|c|}{ Fit Value } & \multicolumn{2}{|c|}{ Lower Error } & \multirow{2}{*}{$\begin{array}{c}\text { Upper Error } \\
+0.16 \\
\end{array}$} \\
\hline 1. & \multicolumn{2}{|l|}{ 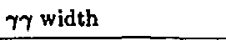 } & 3.01 & keV & \multicolumn{2}{|c|}{-0.15} & \\
\hline 7. & \multicolumn{2}{|l|}{$\mathcal{L}_{\text {eft }}$} & 95.8 & $p b^{-1}$ & \multicolumn{2}{|c|}{-1.1} & +1.1 \\
\hline 8. & \multicolumn{2}{|c|}{$\eta^{\prime}$ background } & 412 & events & \multicolumn{2}{|c|}{-87} & +87 \\
\hline 9. & \multicolumn{2}{|c|}{$K \bar{K}, p \bar{p}$ background } & 342 & events & \multicolumn{2}{|c|}{-34} & +34 \\
\hline 10. & \multicolumn{2}{|c|}{$Q \neq 0$ background } & 147 & events & \multicolumn{2}{|c|}{-50} & +50 \\
\hline 11. & \multicolumn{2}{|l|}{$\varepsilon_{1}^{\pi}$} & .0 .043 & & \multicolumn{2}{|c|}{-0.076} & +0.076 \\
\hline 12. & \multicolumn{2}{|l|}{$e_{2}^{\pi}$} & 0.00 & & \multicolumn{2}{|c|}{-0.05} & +0.05 \\
\hline \multicolumn{8}{|c|}{ Covariance Matrix Correlation Coefficients } \\
\hline & 1 & $\mathbf{7}$ & $\mathbf{8}$ & & 9 & 10 & 11 \\
\hline 7 & -0.052 & & & & & & \\
\hline 8 & 0.109 & -0.049 & & & & & \\
\hline 9 & -0.046 & 0.002 & -0.009 & & & & \\
\hline 10 & -0.003 & -0.022 & -0.009 & & 003 & & \\
\hline 11 & -0.088 & -0.844 & -0.100 & & 004 & -0.032 & \\
\hline 12 & -0.783 & 0.063 & -0.037 & & 001 & -0.002 & -0.154 \\
\hline
\end{tabular}

Note that this fit does include Mennessier's unitarization corrections. However, as we have seen in Chapter 7, those corrections have little effect. Furthermore, the definition used here for $\Gamma_{\gamma \gamma}$ is closer to that of the parameter $\Gamma_{2}\left(m_{j}^{2}\right)$ in the model given by Eqn. 8.1 (Lyth's model) then it is to $\Gamma_{\gamma \gamma}$ as defined by Eqn, 8.2. The unitarization correction is not considered to be part of the coupling of $\gamma \gamma$ to the $\int$. According to Eqn. 8.3, $\Gamma_{2}\left(m_{f}^{2}\right)$ is $0.257 \mathrm{keV}$ less than $\Gamma_{\gamma \gamma}^{2}$. Com- paring the fitted value of $\Gamma_{2}\left(\mathrm{~m}_{f}^{2}\right)$ from Eqn. 8.8 with Eqn. 8.9, we find that the Mennessier result is about $5 \%$ lower. That is to be expected from the comparison of the two unitarized models shown in Fig. 7.9 and corresponds to more than two st andard deviations of the statistical error (the systematic effects are the same for each roodel).

In summary, the two models fit the data equally well. When no unitarization corrections are made, they give results for $\Gamma_{\gamma \gamma}$ which agree with each other within the systematic errors. The principle differences lie in the definitions of $\Gamma_{\Upsilon \gamma}$ when unitarization corrections are made and in the fact that unitarization of the Mennessier model produces little change whereas it lowers by several percent the $f$ peak in the Lyth model.

\subsubsection{Including the Anguiar Distribution}

It is important to consider the effect of a possible helicity-zero contribution to the resonant coupling, since there is no theoretical justification to assume that it is exactly zero. To do so, it is essential to inclue - into consideration the measured angular distribution. We use the distribution of $\cos \theta_{\mathrm{cm}}$ integrated over the range $1.0 \leq W \leq 1.5 \mathrm{GeV}$, in order to be most sensitive to the angular distribution of pion pairs coming from the $f$ resonance.

First, let us consider the angular distribution alone. There are twelve bins of width 0.05 from $\cos \theta_{\mathrm{cm}}$ to the detector limit of $\cos \theta_{\mathrm{cms}}=0.60$. The predicted distribution is a combination of the flat distribution from the Born lerm interfering with the $d_{\lambda_{0}}^{2}(\theta)$ functions from the resonance decay, plus the approximately $\left(1+\cos ^{2} \theta\right) /\left(1-\cos ^{2} \theta\right)$ distribution from the muon-pair background. The experimental acceptance, given for the most part by the single cut $\cos \theta_{\mathrm{lab}} \leq$ 0.60 , falls sharply from a maximum at $\cos \theta_{\mathrm{cms}}=0$ to zero at $\cos \theta_{\mathrm{cms}}=0.60$ and strongly affects the observed shape. The result of fitting to only the angular distribution is that the data prefer essentially zero contribution from helicity-zero. 
The $90 \%$ confidence level upper limit is

$$
\frac{\Gamma_{\gamma \gamma}^{0}}{\Gamma_{7 \gamma}^{2}}<0.15 \quad \text { (\$0\% confidence). }
$$

It is interesting that this ratio actually is constrained substantially by the invariant-mass distribution alone. It is easy to see why that is so. The spectrum is dominated by the interference effect, which enhances the cross section below resonance and decreases it above. Such an effect does not occur for helicity zero, because the helicity-zero Biorn term is negligible in the resonance region. In fact, if the coupling is assumed to be only helicity zero, the model predicts far too few events below resonance and far too many above, and the best fit to the invariantmass spectrum has $\chi^{2}$ of 150 for 16 bins (the two-photon width for that fit is $6 \mathrm{keV}$ ).

Table 8.3 shows the results of fitting simultaneously to the invariant-mass and angular distributions. Both helicity amplitudes are included, and the unitarization corrections to the helicity-two amplitude are included. Figure $\mathbf{8 . 2}$ compares the fitted spectra to the data. The additional systematic effects, such as the dependence on the range of $W$ included in $\chi^{2}$, are about the same as for the fit with only the invariant mass distribution included. Taking them into account results in

$$
\begin{aligned}
& \Gamma_{\gamma \gamma}=\Gamma_{\gamma \gamma}^{0}+\Gamma_{\gamma \gamma}^{2}=3.42 \pm 0.37 \mathrm{keV} \\
& \Gamma_{\gamma \gamma}^{0} / \Gamma_{\gamma \gamma}^{2}<0.14 \quad(90 \% \text { confidence })
\end{aligned}
$$

Here, Lyth's definition of $\Gamma_{\gamma \gamma}$ (Eqn. 8.3) is assumed.

We have found that the DELCO constraint on the ratio of helicity amplitudes is much better than the Crystal Ball result (compare with Eqn. 1.3). That may be surprising at first, but it is because of the relatively small number of $\pi^{0} \pi^{0}$ events observed by the Crystal Bail collaboration. Their advantage lies in being
Table 8.3- Fit of the complete model of Eqn. 8.1 to the untagged $\pi^{+} \pi^{-}$invariant-

\begin{tabular}{|c|c|c|c|c|c|c|c|}
\hline \multicolumn{3}{|c|}{ Parameter } & \multicolumn{2}{|c|}{ Fit Value } & \multirow{2}{*}{\multicolumn{2}{|c|}{$\begin{array}{c}\text { Lower Error } \\
-0.22 \\
\end{array}$}} & \multirow{2}{*}{$\begin{array}{c}\text { Upper Error } \\
+0.27 \\
\end{array}$} \\
\hline \multicolumn{3}{|c|}{ 1. $\Gamma_{\gamma \gamma}$} & 3.51 & $\mathrm{keV}$ & & & \\
\hline \multicolumn{3}{|c|}{ 2. $\Gamma_{\gamma \gamma}^{0} / \Gamma_{\gamma \gamma}^{2}$} & 0.01 & & \multicolumn{2}{|l|}{-0.01} & +0.13 \\
\hline \multicolumn{3}{|c|}{ 4. $\Im g_{2}\left(m_{f}^{2}\right)$} & 0.00026 & $\mathrm{GeV}^{2}$ & \multicolumn{2}{|c|}{-0.00007} & $+0.0 n \operatorname{sen} 7$ \\
\hline \multicolumn{3}{|c|}{ 5. $f$ mass } & 1.266 & $\mathrm{GeV}$ & \multicolumn{2}{|c|}{-0.005} & \\
\hline \multicolumn{3}{|c|}{ 6. full width } & 0.168 & $\mathrm{GeV}$ & \multicolumn{2}{|c|}{-0.012} & 0.011 \\
\hline \multicolumn{3}{|c|}{ 7. $\mathcal{L}_{\text {eff }}$} & 100.8 & $n t-1$ & & +1.2 \\
\hline \multicolumn{3}{|c|}{ 8. $\eta^{\prime}$ backround } & 416 & events & -82 & & +82 \\
\hline \multicolumn{3}{|c|}{ 9. $K \bar{K}, p \bar{p}$ background } & 342 & events & -34 & \multicolumn{2}{|r|}{+34} \\
\hline \multicolumn{3}{|c|}{ 10. $Q \neq 0$ background } & 150 & events & -50 & \multicolumn{2}{|r|}{+50} \\
\hline \multicolumn{3}{|c|}{ 11. $\varepsilon_{1}^{\pi}$} & \multicolumn{2}{|l|}{-0.070} & \multicolumn{2}{|c|}{$-0,085$} & $+0,094$ \\
\hline \multicolumn{3}{|c|}{ 12. $\varepsilon_{2}^{\pi}$} & \multicolumn{2}{|l|}{-0.002} & -0.050 & & +0.050 \\
\hline & & Covarian & e Matrix Co & orrelation & Coefficien & & \\
\hline & 1 & 2 & 4 & 5 & 6 & 7 & 11 \\
\hline 2 & 0.374 & & & & & & \\
\hline 4 & 0.133 & -0.038 & & & & & \\
\hline 5 & -0.106 & -0.286 & 0.099 & & & & \\
\hline 6 & -0.537 & -0.060 & 0.031 & -0.380 & & & \\
\hline 7 & 0.041 & 0.116 & 0.003 & -0.251 & 0.062 & & \\
\hline 11 & -0.265 & 0.091 & -0.016 & 0.216 & 0.224 & -0.781 & \\
\hline 12 & -0.641 & 0.034 & 0.005 & 0.124 & 0.067 & 0.030 & -0.035 \\
\hline $\begin{array}{l}\text { Fil } \\
\chi^{2}\end{array}$ & $\begin{array}{l}\text { nge: } \\
7.3\end{array}$ & $W<1.4$ & $\mathrm{GeV}, \mid \cos \theta_{\mathrm{c}}$ & $\mathrm{ma} \mid \leq 0.60$ & $28 \mathrm{~b}$ & & \\
\hline
\end{tabular}
mass and angular distributions. Definitions of the parameters may be found in Section 8.2. 

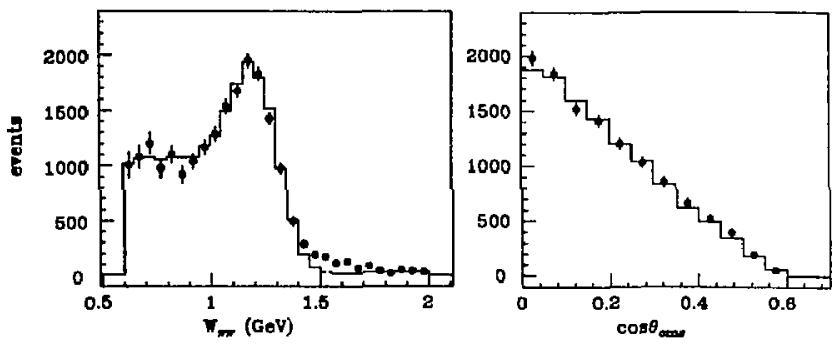

Figure 8.2. Best simultaneous fit to the $W$ and $\cos \theta_{\mathrm{cm}}$ distributions of the untagged $\pi^{+} \pi^{-}$data. The points with error bars are the data. The values of the fitted parameters are given in Table 8.3.

able to observe the region $\left|\cos \theta_{\mathrm{cms}}\right|>0.80$, where the angular distributions differ greatly. However, their error bar in that region is too large to provide a very good constraint. The region near $\left|\cos \theta_{\mathrm{cmu}}\right|=0.70$ is not very helpful, since it is around there that the angular distributions cross. Thus DELCO is not at a disadvantage due to ita acceptance limit of $\left|\cos \theta_{c \mathrm{~ms}}\right|<0.60$. Also, the Crystal Ball limit may be more stringent if the $\lambda=1$ fraction (which must be negligible for an untagged experiment) were not included in the fit.

If the ratio $\Gamma_{\gamma \gamma}^{0} / \Gamma_{\gamma \gamma}^{2}$ is fixed at the Crystal Ball 1- $\sigma$ upper limit of 0.51 , then the best fit has a $\chi^{2}$ from the 12 bins of the angular distribution of 16 , which is an increase of 9 units from the best fit with the ratio left free. This increase in $x^{2}$ comes from a systematic deviation which is quite obvious when plotted--the Monte Carlo angular distribution with the helicity-zero contribution mixed in falls too rapidly in the region $0.2<\left|\cos \theta_{\mathrm{cms}}\right|<0.6$ relative to the data. Part of the reason for such a good constraint is that all the systematic problems, such as the pion-pair trigger efficiency, have a small effect on the observed angular distribution relative to the effect of the actual decay angular distribution. The major experimental effect is the detector angular acceptance, which is weli understood.

\subsubsection{Extrapolating $\Gamma_{\gamma \gamma}$ to $Q^{2}=0$}

For the energy range subtended by the $f$ peak, the EPA luminosity function predicts an average $Q^{2}$ of $\overline{Q^{2}}=0.006 \mathrm{GeV}^{2}$, where $Q^{2}$ is defined to be the maximum of $-q_{i}^{2}$ for the two photons. To extrapolate the results of the untagged analysis for the two-photon width to $Q^{2}=0$, we assume the GVDM form factor of Eqn. 2.20. It predicts that all the untagged results for $\Gamma_{\gamma \gamma}$ sho'ild be multiplied by a factor of 1.014. This change is small compared with the experimental errors, which are $11 \%$ or more, so it is not critical whether or not such a correction is made.

\subsection{Fit RESULTS FOR THE TAGgED ANALYSIS}

For the tagged events, the principle interest is to see the $Q^{2}$ dependence of the two-photon width of the $f$. To this end, we ignore for now the question of the unitarity orrection and simply compare the result for a simple model of interfering Born plus Breit Wigner amplitudes with the equivalent result obtained at $Q^{2}=0$. The question of the ratio of helicity contributions takes on a greater importance, however. The average $Q^{2}$ for events with a single tag in the DELCO luminosity counters is calculated by Monte Carlo from Eqn. 2.18 to be $\overline{Q^{2}}=0.44 \mathrm{GeV}^{2}$. The authors of Ref. 66 give predictions, based on a non-relativistic quark model, for the $Q^{2}$ evolution of the three possible helicity amplitudes of the cross section for $\gamma \gamma \rightarrow f$. At $Q^{2}=0$ the $\lambda=1$ cross section is, of course, zero, and the $\lambda=0$, cross section is zero as well. But for an experimenl with a minimum tagging angle of 0.25 milliradians, the $\lambda=0$ contribution is $5 \%$, and the $\lambda=1$ contribution is $25 \%$.

'To model the continuum we continue to use the same Born term as given in Fqn. 7.27, except that it is multiplied by the GVDM form factor of Eqn. 2.20. This is justified by the fact that the result gives a reasonable fit below the $f$ peak The first bin, from $W=0.6 \mathrm{GeV}$ to $W=0.65 \mathrm{GeV}$, is an exception. It appears to be a factor of two too low, unless either the cross section or the 
detector acceptance has some unknown sharply changing behavior at that point, but a statistical fluctuation cannot be ruled out.

Experiments in the past ${ }^{5,3}$ commonly have analyzed tagged data with the assumption that the $f$ resonance is produced only in helicity two. But then the results for the $Q^{2}$ dependence often are compared with the GVDM form factor, which is inconsistent. GVDM explicitly includes contributions for which one of the colliding photons is longitudinally polarized, which are suppressed only by factors of $Q^{2} / m_{V}^{2}$ (see Eqn. 2.20) relative to the transverse-transverse contributions. These additional contributions necessarily have helicity one. That is not a serious issue for the description of the continuum, but to measure the resonant cross section accurately within a limited angular acceptance, one must assume the proper angular distribution for the resonance decay. A Monte Cariu calculation of the resonance term m!ltiplied by the GVDM form factor predicts that on average for the DELCO tagging acceptance

$$
\frac{\sigma_{L T}}{\sigma_{T T}}=\frac{\Gamma_{\gamma \gamma}^{1}}{\Gamma_{\gamma \gamma}^{0}+\Gamma_{\gamma \gamma}^{2}}=0.081 .
$$

Furthermore, it is safe to assume within this acceptance that the transverse polarization parameter $\varepsilon$ of the $\gamma \gamma$ luminosity function (Eqn. 2.16) simply is unity.

The parameters in the fit differ only signtly from those presented in Sec...n 8.2 for the untagged analysis. The background from $K^{+} K^{-}$and $p \bar{p}$ is negligible in this case and is ignored. The other parameters which differ from the untagged analysis are

$$
\begin{gathered}
\mathcal{L}_{\text {eft }}=94.1 \pm 3.3 \mathrm{pb}^{-1}, \\
N_{\eta^{\prime}}=72 \pm 16 \text { events, } \\
N_{\text {had }}=550 \pm 112 \text { events. }
\end{gathered}
$$

In addition, the tagged analysis makes use of the helicity-one coupling. The helicity-one contribution is not shown in Eqn. 8.1, but it differs from the other two resonance terms only in the angular distribution. For siraplicity, the continuum production is assumed not to interfere with the helicity-one resonance amplitude, even though using the GVDM form factor implies some helicity-one contribution in the continuum.

First, let us see how well the data can constrain the helicity ratios. When all three helicity contributions are allowed to vary independently, the result is

$$
\begin{gathered}
\Gamma_{77}=1.42 \pm 0.33 \mathrm{keV}, \quad\left(\overline{Q^{2}}=0.44 \mathrm{GeV}^{2}\right), \\
\Gamma_{7 \gamma}^{0}: \Gamma_{7 \gamma}^{1}: \Gamma_{7 \gamma}^{2}=40: 0: 60 .
\end{gathered}
$$

Included in the systematic error are the effects of changing the range of $W$ included in the fit, but for the tagged data the results are not very sensitive to that change. In fact, even the prediction above the $f$ peak agrees well with the data, partly because the statistical errors ase large compared with the untagged data. The explicit results of sush a fit, with the $W$ range restricted to $0.65<W<1.4 \mathrm{GeV}$, are shown in Table 8.4, and Fig. 8.3. The data prefer that there be no helicity-one contritution. If the fit is repeated with the helicity ratios fixed to the prediction of Ref. 66, then $\chi^{2}$ for the twelve $\cos \theta_{\text {cme }}$ bins increases from 4.8 to 10.7 , so that prediction is not well supported by the data. On the other hand, the GVDM prediction of $8 \%$ for the $\lambda=1$ contribution is consistent with the fit shown in Table 8.4. The fit does not constrain very well the ratio of the $\lambda=0$ to $\lambda=2$ contributions, since their angular distributions within the acceptance are relatively similar. If the $\lambda=1$ contribution is taken from GVDM and the $\lambda=0$ contribution from Ref. 66, and if the result is fit only over the mass spectrum, then $\chi^{2}$ for the angular distribution is 7.3 for 12 bins. The best fit for the two-photon width then is found to be

$$
\begin{gathered}
\Gamma_{\gamma \gamma}=1.16 \pm 0.18 \mathrm{keV}, \quad\left(\overline{Q^{2}}=0.44 \mathrm{GeV}^{2}\right), \\
\Gamma_{\gamma \gamma}^{0}: \Gamma_{\gamma \gamma}^{1}: \Gamma_{\gamma \gamma}^{2}=50: 75: 875 .
\end{gathered}
$$


Table 8.4. A fit to the tagged $\pi^{+} \pi^{-}$data with all three helicity amplitudes allowed to vary independently. Complete definitions of the parameters may be found in Section $\mathbf{8 . 2}$.

\begin{tabular}{|c|c|c|c|c|c|c|c|}
\hline \multicolumn{3}{|c|}{ Parameter } & \multicolumn{2}{|c|}{ Fit Value } & \multirow{2}{*}{$\begin{array}{c}\text { Lower Error } \\
-0.29 \\
\end{array}$} & \multirow{2}{*}{\multicolumn{2}{|c|}{$\frac{\text { Upper Error }}{+0.34}$}} \\
\hline 1. & \multicolumn{2}{|l|}{$\Gamma_{\boldsymbol{\gamma \gamma}}$} & 1.40 & $\mathrm{keV}$ & & & \\
\hline 2. & \multicolumn{2}{|l|}{$\Gamma_{\gamma \gamma}^{0} / \Gamma_{\gamma \gamma}^{2}$} & 0.68 & & -0.66 & & +1.09 \\
\hline 3. & \multicolumn{2}{|l|}{$\Gamma_{\gamma \gamma}^{1} / \Gamma_{\gamma \gamma}$} & 0.00 & & -0.00 & & +0.13 \\
\hline 5. & \multicolumn{2}{|l|}{$f$ mass } & 1.267 & $\mathrm{GeV}$ & -0.010 & & +0.010 \\
\hline 6. & \multicolumn{2}{|c|}{ full width } & 0.172 & $\mathrm{GeV}$ & -0.019 & & +0.018 \\
\hline 7. & \multicolumn{2}{|c|}{$\mathfrak{L}_{\text {eff }}$} & 92.3 & $p^{b^{-1}}$ & -2.3 & & +2.3 \\
\hline 8. & \multicolumn{2}{|c|}{$\eta^{\prime}$ background } & 72 & events & -16 & \multicolumn{2}{|r|}{+16} \\
\hline 10. & \multicolumn{2}{|c|}{$Q \neq 0$ background } & 538 & events & -112 & \multicolumn{2}{|r|}{+112} \\
\hline 11. & \multicolumn{2}{|c|}{$\varepsilon_{1}^{\pi}$} & \multicolumn{2}{|l|}{-0.001} & -0.095 & & +0.095 \\
\hline 12. & \multicolumn{2}{|l|}{$\varepsilon_{2}^{\pi}$} & \multicolumn{2}{|l|}{-0.002} & -0.050 & & +0.050 \\
\hline \multicolumn{8}{|c|}{ Covariance Matrix Correlation Coefficients } \\
\hline & 1 & 2 & $\mathbf{3}$ & 5 & 6 & 7 & 11 \\
\hline 2 & \multicolumn{2}{|l|}{0.876} & & & & & \\
\hline 3 & 0.066 & -0.033 & & & & & \\
\hline 5 & -0.464 & 0.504 & -0.005 & & & & \\
\hline 6 & 0.245 & 0.110 & 0.120 & 0.152 & \multirow[b]{2}{*}{-0.094} & & \\
\hline 7 & 0.005 & 0.278 & -0.021 & -0.113 & & & \\
\hline 11 & \multirow{2}{*}{$\begin{array}{r}0.046 \\
-0.211\end{array}$} & -0.011 & -0.234 & 0.144 & 0.132 & -0.443 & \\
\hline 12 & & 0.000 & -0.026 & 0.053 & 0.018 & -0.029 & -0.103 \\
\hline & $\begin{array}{l}\text { ange: } \\
1.4\end{array}$ & 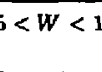 & $40 \mathrm{GeV}$, & $\cos \theta_{\mathrm{cms}} \mid \leq$ & 0.60 & bins & \\
\hline
\end{tabular}
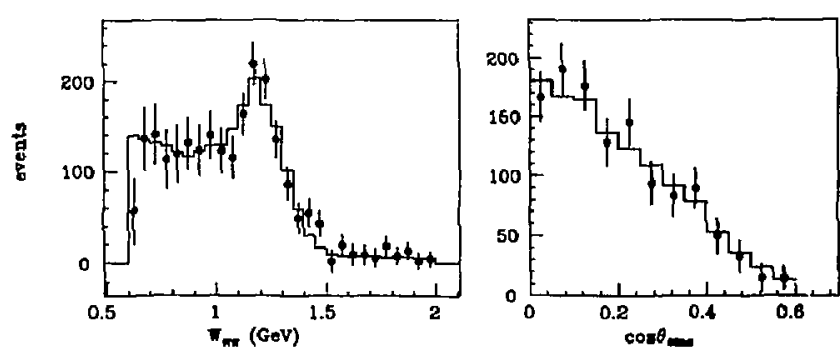

Figure 8.3. Complete fit of the tagged $\pi^{+} \pi^{-}$data with all three helicity amplitudes allowed to vary independently. The results of the fit are detailed in Table 8.4.

8.5 REMARKS ON THE FITTED $f$ Miass

Recall that in Section 7.4.2 we have predicted that the energy dependence of the resonance requires that the parameter $m$ g actually be $1.284 \mathrm{GeV}$ in order that the pole in the complex plane falls at the point specified in Ref. 6 . Thus the best values for $m$, presented in the fits of this chapter could be typically $2 \%$ low. If that really is so, ther it actually is consistent with the $K$, mass peak, which is observed to be $2 \%$ low (see Section 3.1). The fitted value of $r_{\gamma \gamma}$, however, is not sensitive to the assumption made fot $\sigma_{m}$. The important point is that the urcertainty in the momentum scale does not affect the normalization of the $\mu^{+} \mu^{-}$subtraction, because the normalization is derived from a measurement of $\mathrm{e}^{+} \mathrm{e}^{-} \rightarrow \mathrm{e}^{+} \mathrm{e}^{-} e^{+} e^{-}$, which is affected exactly the same by systematic shifts of the momentum scale as arc the measurements of muon and pion pairs.

In this chapter we have seen the results of many fits for the two-photon width of the $f$ under various theoretical and experimental assumptions. In Chapter 10 the implications of these results are discussed, and the unfolded spectro for the untagged pion pairs are presented. 


\section{The Time-of-Flight Analysis}

The analysis of kaon and proton pairs differs from that of pion pairs only in that the time-of-flight counters must be used in addition to the other systems. The trigger is the same, although the response to the trigger differs. The tracking is the same, though for kaom it is essential to include in the Monte Carlo simulation the effects of in-flight weak decays. And the Cerenkov counters are used in the same manner to reject electron pairs, although when analyzing kaons and protons they are not as important, since the time-of-flight analysis would reject most electrons anyway. So up to the point of actually analyzing the timing information, the analysis is almost identical to that of the pion pairs. The main exception jo that the lower cut on the invariant wass is changed to $W_{K K}>1.3 \mathrm{GeV}$, and no explicit upper cut is made. This chapter first discusses the performance of the time-offight system and how best to extract the maximum amount of information from it The resulting analysis then is used to measure the cross sections for $\gamma \gamma \rightarrow K^{+} K^{-}$ and $\boldsymbol{\gamma} \boldsymbol{\gamma} \rightarrow \boldsymbol{p} \bar{p}$.

The system consists of 52 plastic scintillators, $2.5 \mathrm{~cm}$ thick and of varying widths and lengths, mounted on the faces of the six aluminum boxes which house the barrel shower counter system and are arranged in a hexagon about the inner detector. Most of the counters actually are longer than the barrel shower counters, but two are cut a few centimeters shorter because of obstructions. Also, in addition to the gaps between sextants, there are a some $\phi$ gaps which do not appear in the shower counters. Therefore, within the range $-0.6<\cos \theta<0.6$, the time-of-flight acceptance is slightly smaller than that of the shower counters. The tracking is not used to define strictly the acceptance. Instead, since there are only two well separated tracks in each event, a fired counter is assumed to be associated with a track if the track passes near to it and there is a good time reading at both ends of the counter.
9.1 Calibration of THE TIMe-of-Flight System

Calibration of the counters is done using the low-energy electrons from twophoton events. In fact, the detector was allowed to be triggered by events with only a single electron detected (usually produced by the process $e^{+} e^{-} \rightarrow e^{+} e^{-} e^{+} e^{-}$), so there is an abundant supply of such electrons for every few hours of data taken. That allows one to remove slight fluctuations in the timing which occurred on the time scale of a few days or less. Large fluctuations which occurred on time scales of several days or more divide the data into 30 run blocks, for which the entire calibration was done separately for each.

Muons and pions, also from two-photon events, also were used in the calibration, but only for measuring light attenuation and pulse-height gain. The corrected pulse height for a particular phototube is calculated from the raw pulse height by the formula

$$
a_{c}=a_{r} \cdot g_{i} \cdot e^{\Delta x / \lambda_{j}},
$$

where $g_{i}$ is the pulse-height gain for the ith phototube, $\Delta z$ is the distance from the hit to the phototube, and $\lambda_{j}$ is the attenuation length for the $j$ th scintillator. For each run block, the $g_{i}$ and $\lambda_{j}$ were adjusted such that the corrected pulse-height distributions from minimum-ionizing particles all consisted of relatively narrow peaks centered about a pulse height which is independent of the phototube and its distance from the position of the hit. Evidence of large var:ations in counter equality is found in the measured attenuation lengths, which vary from $70 \mathrm{~cm}$ to $270 \mathrm{~cm}$.

Then, using electrons, plots were made of the average time residual for each phototube versus the measured pulse height and the distance of the electron hit from the tube, as measured by tracking. The time residual is the measured time minus the time predicted by tracking. Since the electrons travel with essentially the velocity of light, the predicted time is $t_{p}=t_{0}+\ell / c+\Delta z / v_{i}$, where $t_{0}$ is the time of the beam crossing, $\ell$ is the measured arc length from the interaction point to the counter, and $v_{i}$ is the effective velocity of light in the scintillator. Corrected 
times sre determined from the measured times by adding a consta it pedestal plus a term in $1 / \sqrt{a_{r}}$ to correct for pulse-height slewing, where $a_{r}$ is the uncorrected pulse height. An additional polynomial correction is made for very large pulse heights (greater than 15 times minimum ionizing), since there the $1 / \sqrt{a_{r}}$ behavior no longer halds. Also, a nonlinear correction is made for the $z$ dependence when the hil is near the phototube, because in that region the $z$ dependence cannot be fully described by a constant effective velocity. All of these constints for the pulse-height slewing corrections, non-linear $z$ dependence, the pedestals, and the $v_{\text {; }}$ were adjusted iteratively for euch phototube or scintillator until the time residuals formed a peak centered about zero and as narrow as possible.

\subsection{Monte Carlo smulation}

The performance of the various componsnts of the time-of-flight gystem must be measured in order to calibrate the Monte Carlo simulation. The sarne results also are needed in order to calculate probability weights for particle identification. The firat priority is that the Monte Carlo simulate the pulse height distribution well. There is no interest in measuring the velocity of electrons, so only the heavy particles need to be handled properly.

For heavy charged particles, the energy deposit in the scintillator is approximately given by ${ }^{67}$

$$
\frac{d E}{d x} \propto \frac{1}{\beta^{2}}\left[\ln \left(\frac{2 m_{e} \eta^{2} c^{2}}{I}\right)-\beta^{2}\right],
$$

where $I$ is a phenomenological constant, $\beta c$ is the incident particle's velocity, and $\eta^{2}=\beta^{2} /\left(1-\beta^{2}\right)$. Fluctuations about this mean energy deposit are given by a Landau distribution; 68 which forms a peak with a width almost an order of magnitude more narrow than the resolution of the counter system and with a long, low tail extending to pulse heights more than twice as large as that of the peak. The proportionality constant for Eqn. 9.2 is measured for each counter from the position of the peak in a histogram of pulse heights, corrected for attenuation and the incident angle, of minimum-ionizing muons and pions. Also measured for cach

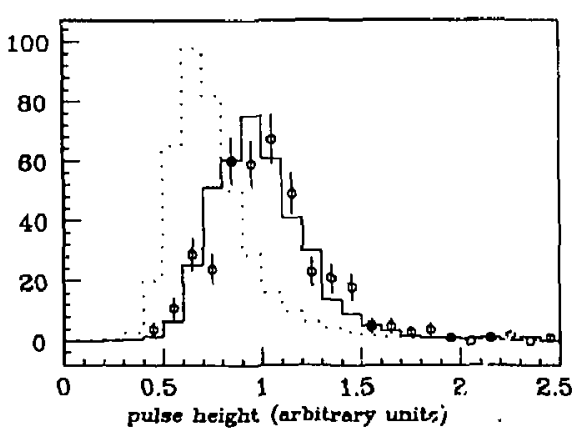

Figure 9.1. Energy deposit of kaons in the time-of-flight counters. The points with error bars show the corrected pulse height distribution for time-of-fight identified kaons, and the so'ic histogram is the Monte Garlo prediction. The dotted histogram sho\%s the distribution for minimumionizing muons and pions, for which the counters were calibrated. The units of the abscissa are arbitrary.

phototube is the width of the pulse height distribution. In the Monte Carlo, the energy deposit is calculated from the path length through the counter by Eqn. 9.2. It then is smeared by a random number drawn from the Landiu distribution, attenuated according to the distance from the hit to the tube, and smeared by a random number drawn from a gaussian distribution of the appiopriate width. Figure 9.1 shows that the Monte Carlo performs well when predi ting the pulse height distribution for incident kanns.

Next, the time resolution must be simulated. There are not enough data to measure accurately the pulse-height dependence of the resn ution for each phototube. Instead, what is done is to make a histogram of the time residual of each phototube using only those electrons which produce a pulse height greater than $0.5 g x$, where $g x$ is an arbitrary unit defined by the calibration. Since above that cutoff the resolution is almost constant, then the resulting histograms are well 


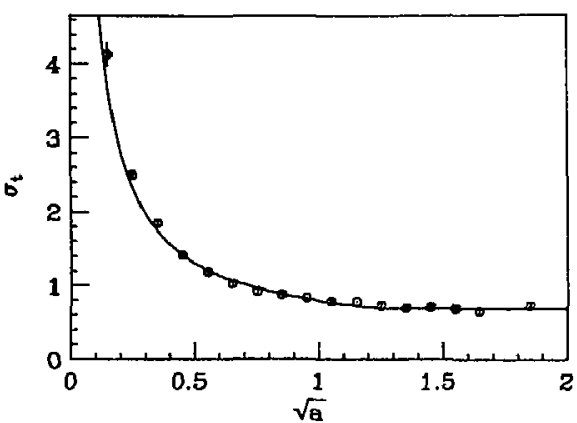

Figure 9.2. The pulse-height dependence of the resolution of the time-offlight counters. The points show the gaussian widths of the distribution of time residuals divided by phototube-dependent widths. Hence the units of the ordinate are arbitrary. The smooth curve is the best fit to the parameterization described in the text.

fitted by gaussian curves. Then a new set of histograms is made by combining all phototubes and accumulating the time residual divided by the sigma of the gaussian fit for the particular phototube. One histogram is made for each of several ranges of pulse height, and each is fit to a gaussian curve. Figure 9.2 shows a plot of the resulting gaussian widths versus the square-root of the pulse height. The smooth curve is a fit to a constant plus $1 / \sqrt{a_{r}}$, changing to a straight line at about $1.5 \mathrm{gx}$. This parameterization is what is used in the Monte Carlo simulation and the analysis. As a check of the method, Fig. 9.3 shows a histogram of the time residual divided by the expected resolution for a sample of high-energy muons from the process $e^{+} e^{-} \rightarrow \mu^{+} \mu^{-}$. The Monte Carlo agrees well with the data, and both samples are well fitted by a gaussian curve of width close to unity and mean close to zero.

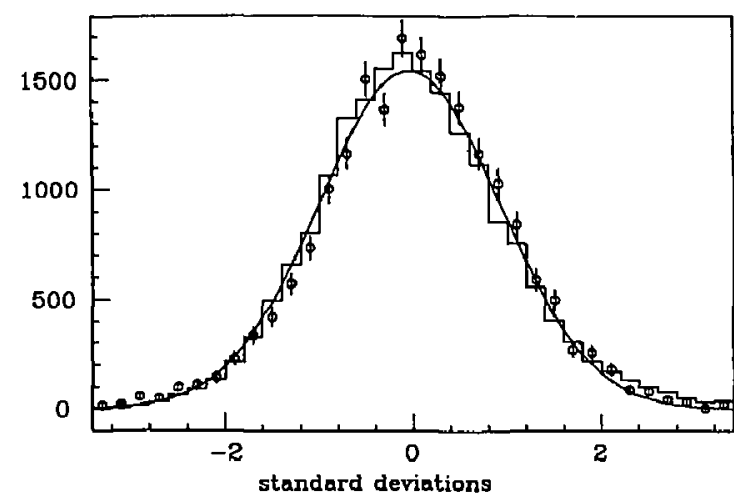

Figure 9.3. The time-of-fight phototube time resolution for $14.5 \mathrm{GeV}$ muons, comparing data (solid histogram) with Monte Carlo (points with error bars). What are plotted are histograms of the time residual divided by the expected resolution. The smooth curve is a fitted gaussian curve with is width of $\sigma=0.964$ and a mean of $\mu=-0.037$.

9.3 Time Consistency and the Timing Resolution

The time-of-flight analysis relies heavily on tracking information. Especially the momentum measurement is important, because it must be used along with the time-of-light in order to identify kaons and protons. The tracking cuts of the pion-pair analysis are used again here, but with the addition of one cut. Both tracks in each event are required to have a $\chi^{2}$ per degree of freedom less than 2.5 for the fit to the drift chamber hits. This helps to reduce the incidence of measurements with large non-gaussian errors. However, the most powerful cut in that respect is one made on the timing information itself. The time is measured independently at both ends of the counter, so it is possible to have a cut which requires the two times to be consistent without biasing the average. 
Let $t_{1}$ be the time residual measured by the southern end of the counter and $t_{2}$ the time residual from the northern end, where time residual is defined to be the difference between the measured time and the time expected for a particle traveling the speed of light. With $\Delta t \equiv t_{1}-t_{2}$, the time cunsistency is defined to be $\Delta t / \sigma_{\Delta l}$. The main contributions to the error estimate $\sigma_{\Delta l}$ are the timing uncertainties, $\sigma_{t_{1}}$ and $\sigma_{t_{2}}$, which are caleulated from the measured pulse height. by using the parameterization shown in Fig. 9.2. There also is a contribution from tracking which introduces a small anticorrclation between $t_{1}$ and $t_{2}$. The error in the prediction for the $z$-position of the point of impact of the particle on the time-of-flight counter (denoted by $z_{t}$ ) comes primarily from errors in the tracking parameters $z_{0}$ and $\tan \lambda . \lambda$ is the dip angle of the track, and $z_{0}$ is the $z$ coordinate of the track at the point nearest the beam line. The error estimate for $z_{t}$ is expressed in terms of the tracking covariance matrix by

$$
\sigma_{x_{i}}^{2}=\sigma_{x_{0}}^{2}+\rho_{s f}^{2} \sigma_{\tan \lambda}^{2}+2 \rho_{\mathrm{eff}} \sigma_{x_{0}, \tan \lambda}^{2}
$$

The parameter $\rho_{\text {eff }}$ is the effective moment arm between where the measurement occurs (in the drift chambers-not at the origin) and the counter. It has been found from Mon te Carlo to be approximately half of the actual arc length. Finally, the error estimate for the time consistency is

$$
\sigma_{\Delta t}^{2}=\sigma_{t_{1}}^{2}+\sigma_{t_{2}}^{2}+4 \sigma_{z_{t}}^{2} / u_{i}
$$

Figure 9.4 shows a histogram of $\Delta t / \sigma_{\Delta t}$ for the entire sample of non-electron pairs used for time-of-flight analysis. The histogram is well fit by a gaussian curve with $\sigma=1.05$ and $\mu=0.003$, verifying the validity of the error estimates. Before continuing the aralysis, all events are rejected which do not have two tracks with $\Delta t / \sigma_{\Delta t}<2.5$. Outside the $2.5 \sigma$ limit the gaussian curve begins to fall well below the data, which have a long tail extending as far as $\pm 10 \sigma$. This cut reduce: the data sample by about $5 \%$.

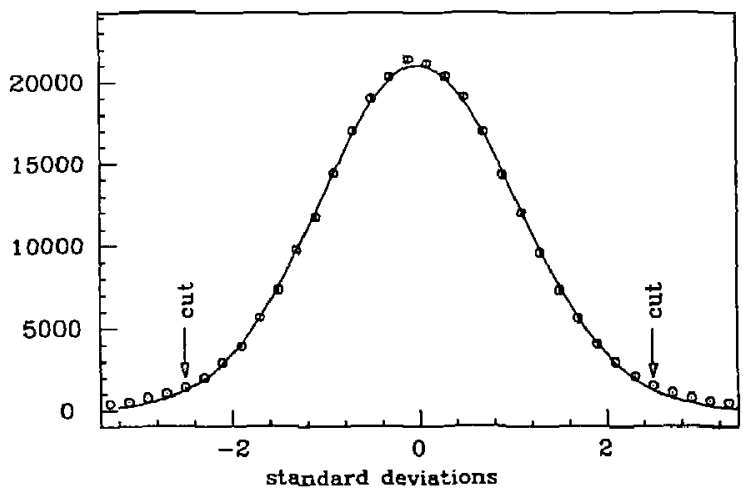

Figure 9.4. Histogram of the time consistency of the two measurements of the time residual made for each minimum-ionizing particle hitting time-of-flight counter. Each entry of $t_{1}-t_{2}$ is divided by the expected error, as calculated from Eqn. 9.4. The smooth curve is a gaussian fit.

Two more cuts ire made on the data to reject tracks with inordinately large expected timing errors. Four of the counters consistently give time residuals more than twice as large as those of all the remaining counters, so events with tracks hitting those counters are not used. And those tracks which have a corrected pulse height, averaged over both phototubes, less than $0.2 g x$ are rejected because of the large timing errors expected from such a small pulse height. Also, events including tracks with pulse heights greater than $5.0 \mathrm{gx}$ are rejected because they are suspected of being associated with some sort of noise contribution.

The best value for the time residual is a weighted average of $t_{1}$ and $t_{2}$ :

$$
\bar{t}=\left(\frac{1}{\sigma_{t_{1}}^{2}} t_{1}+\frac{1}{\sigma_{t_{1}}^{2}} t_{2}\right) \cdot \sigma_{l}^{2}, \quad \sigma_{k}=\left(\frac{1}{\sigma_{t_{1}}^{2}}+\frac{1}{\sigma_{t_{2}}^{2}}\right)^{-1} .
$$

Figure 9.5 shows a histogram of $\vec{t}$ for high-energy muons, One does not expect 


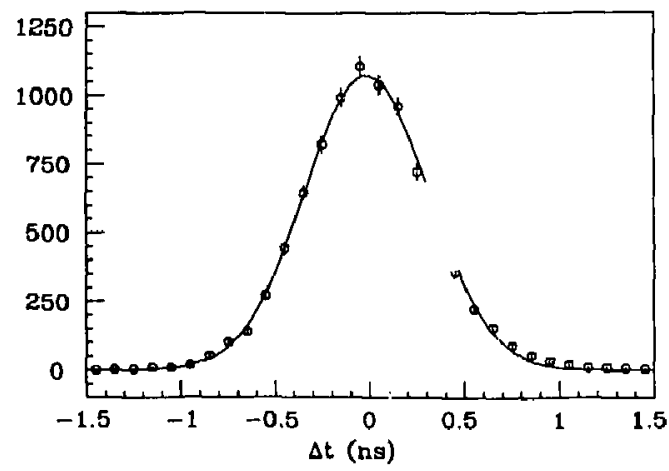

Figure 9.5. The time-of-flight resolution. The histogram is of the time residuals measured from $14.5 \mathrm{GeV}$ muons, and the smooth curve is a gaussian fit.

the shape to be truly gausbian since the entries come from samples with varying resolution. However, as one can see, a gaussian curve does not fit too badly, and its width, $\sigma=0.327 \mathrm{~ns}$, can be considered to be the average time resolution of the system. When a histogram is accumulated with the time residuals divided by the expected error, then the gaussian fit is excellent. One finds that $0.50 \%$ of the entries are outside of the $\pm 3 \sigma$ range, compared with $0.26 \%$ for a true gavssian distribution. Thus the non-gaussian tails are well suppressed.

Consider now the two-photon data set, which contains kaons and protons as well as muons and pions. The difference in time of flight between particle types decreases as their momentum ircreases. This can be seen clearly in Fig. 9.6, which shows \& scatler plot "f the inverse of the measured velocity as a function of the measured momentum. Buth tracks are included from all events which have passed all analysis cuts except for cuts on the time of flight itself. It is clear that kaons can be statistically separated from pions only in the region below $p \approx 1 \mathrm{GeV}$.

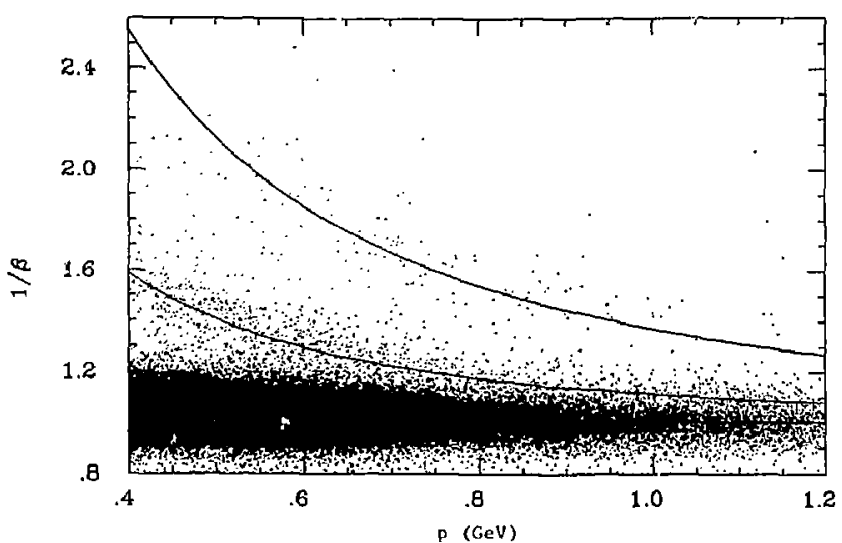

Figure 9.6. The momentum versus the inverse of the measured velocity for tracks from events of the two-photon data set. The smooth curves show the expected trajectories for muons, pions, kaons, and protons.

\subsection{Mass Determination and Event Weights}

The time-of-fight mass measurement is calculated from the measured momentum and time by

$$
m^{2}=p^{2}\left(\frac{1}{\beta^{2}}-1\right),
$$

where $\beta c=\ell /(\bar{t}+\ell / c)$ is the measured velocity. The error in the momentum measurement comes primarily from errors in the curvature $x$ and the dip angle $\lambda$. The correlation between $\kappa$ and $\lambda$ is negligible, so we calculate from $p=\sqrt{1+\tan ^{2} \lambda} / x$ the linear propagation of errors:

$$
\begin{gathered}
\sigma_{p}^{2}=\left(\frac{\partial p}{\partial \kappa}\right)^{2} \sigma_{\kappa}^{2}+\left(\frac{\partial p}{\partial \tan \lambda}\right)^{2} \sigma_{\tan \lambda}^{2}, \\
\frac{\partial p}{\partial \kappa}=-\frac{p}{\kappa}, \quad \frac{\partial p}{\partial \tan \lambda}=\frac{1}{\kappa} \frac{\tan \lambda}{\sqrt{1+\tan ^{2} \lambda}} .
\end{gathered}
$$


The error estimate for $m^{2}$ is given by

$$
\begin{gathered}
\sigma_{m^{2}}^{2}=\left(\frac{\partial m^{2}}{\partial p}\right)^{2} \sigma_{p}^{2}+\left(\frac{\partial m^{2}}{\partial t}\right)^{2} \sigma_{l}^{2}, \\
\frac{\partial m^{2}}{\partial p}=\frac{2 m^{2}}{p}, \quad \frac{\partial m^{2}}{\partial t}=\frac{2 p^{2} t c}{l^{2}} .
\end{gathered}
$$

Figure 9.7 demonatraien that mass resolution is properly calculated and that the errors are very close to being frem gaussian distributions. The histogram is of the quantity $\left(m^{2}-m_{\mu}^{2}\right) / \sigma_{m^{2}}$, so one expects a gaussian shape centered about zero and of unit width. $\dagger$ That is found to be the case to within a few percent of one standard deviation. From the plot with a logarithmic scale, one can see well the non-gaussian taile in the data. They are very small. However, the overall number of muons and pions compared with kaons is enormous, ac the tails remain a problem. For the most part, their effects can be avoided by requiring both particles in an event to be positively identified as kaons before calling the event a kaon pair. When the $m^{2}$ histogram is accumulated without dividing by the expected resolution for each entry, then a gaussian fit is rather poor, due to a significant variation in the resolition from one event to another. Nonetheless, the $\sigma$ of the fit, $0.037 \mathrm{GeV}^{2}$, can be considered to be approximately the average $\mathrm{m}^{2}$ resolution of the system.

To identify the particle type, a $x^{2}$ value is calculated for each of the four possible mass hypotheses $m_{x}$, where $x \equiv\{\mu, \pi, K, p\}$. The $\chi^{2}$ is calculated by comparing the expected time residual to both of the mersured time residuals:

$$
x_{x}^{2}=\sum_{i, j=1}^{2}\left(t_{i}-t_{x}\right)\left(\sigma^{-1}\right)_{i j}\left(t_{j}-t_{x}\right),
$$

where $t_{x}$ is the expected time residual for a particle of mass $m_{x}$ traveling the are of length $\ell$ and is given by $t_{x}=\left(\ell / \beta_{x} c\right)\left(1-\beta_{z}\right)$, with $\beta_{z}=1 / \sqrt{1+m^{2} / p^{2}}$. The

t About a quarter of the eventa in the sample actually are pion paira. But $m_{\mu}^{2}-m_{\mu}^{2}$ in only about $20 \%$ of the everage realution of $\mathrm{m}^{2}$, which is a relatively minor effect.

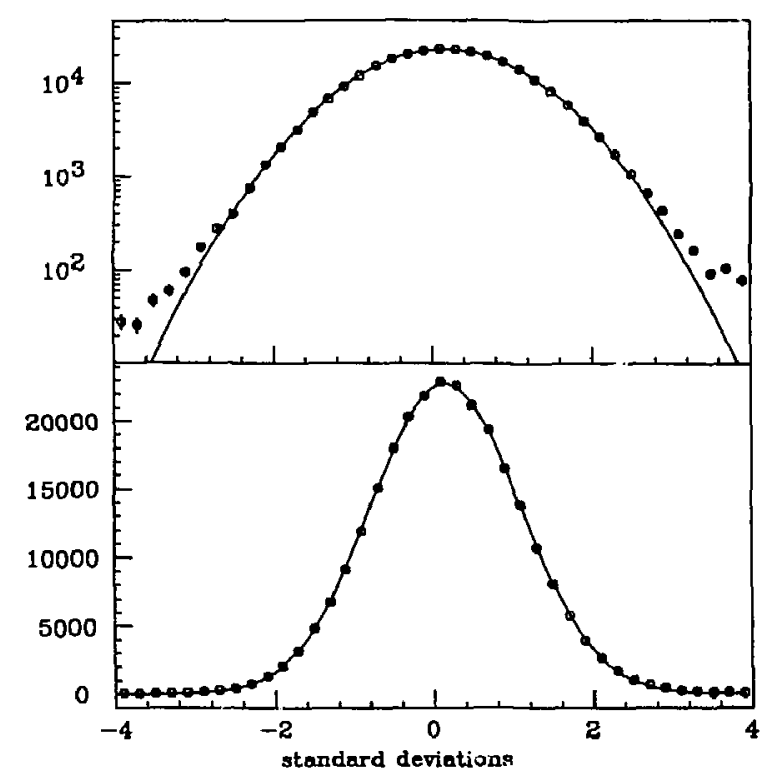

Figure 9.7. The time-of-flight $\mathrm{m}^{2}$ for non-electron pairs. The histogram is of the difference of the time-of-flight $\mathrm{m}^{2}$ and $\mathrm{m}_{\mu}^{2}$, divided by $\sigma_{\mathrm{m}^{2}}$, the expected resolution. The solid curve is gaussian with $\sigma=0.84$ and $\mu=0.16$.

covariance matrix $\sigma$ includes the effects of errors in the two time measurements and the errors from $p, z_{0}$, and $\tan \lambda$. The non-diagonal terms come from only the last two variables, so a $2 \times 2$ derivative matrix is defined:

$$
\left(\begin{array}{ll}
A_{11}=\frac{\partial t_{1}}{\partial z_{0}}=-\frac{1}{v} & A_{12}=\frac{\partial t_{1}}{\partial \tan \lambda}=-\frac{1}{2} T_{x} \beta_{x}^{2} \sin 2 \lambda-\frac{\rho_{\mathrm{ef}}}{v} \\
A_{21}=\frac{\partial t_{2}}{\partial z_{0}}=\frac{1}{v} & A_{22}=\frac{\partial t_{2}}{\partial \tan \lambda}=-\frac{1}{2} T_{x} \rho_{x}^{2} \sin 2 \lambda+\frac{\rho_{\mathrm{efl}}}{v}
\end{array}\right),
$$


where $T_{x} \equiv \ell / \beta_{x} c=t_{x}+\ell / c$. Algo needed are the derivatives of the times with reapect to the track curvature:

$$
\frac{\partial t_{1}}{\partial x}=\frac{\partial t_{2}}{\partial \kappa}=T_{x}\left(1-\beta_{x}^{2}\right) p \cos \lambda
$$

Iet $V_{i j}\{i, j=1,2\}$ be the $2 \times 2$ covariance matrix for $z_{0}$ and $\tan \lambda$, as obtained from track fitting. Then the covariance matrix $\sigma$ takes the form

$$
\begin{aligned}
\sigma_{i i} & =\sum_{j k} A_{i j} V_{j k} A_{i k}+\left(\frac{\partial t_{i}}{\partial \kappa}\right)^{2} \sigma_{\kappa}^{2}+\sigma_{i_{i}}^{2} \\
\sigma_{12}=\sigma_{21} & =\sum_{j k} A_{1 j} V_{j k} A_{2 k}+\left(\frac{\partial t_{1}}{\partial \kappa}\right)^{2} \sigma_{\kappa}^{2}
\end{aligned}
$$

Finally, the weight of a given track is defined for each particle type to be proportional to the gaussian probability that ouch a particle would produce the measured times:

$$
W_{x}=\frac{1}{\sqrt{\operatorname{det}(\sigma)}} \cdot \exp \left(-\frac{1}{2} x_{x}^{2}\right)
$$

The ovent weight is determined by the product of the weights of the two tracks. To normalize it properly, however, it is necessary to determine roughly the relative abundance of the various event types in the data. To do so, a scatter plot is made of $\mathrm{m}^{2}$ of the positively charged particle versus $\mathrm{m}^{2}$ of the negatively charged particle. Figure 9.8 shows such a plot including all data after all analysis cuto have been made, excepting those cuts made on the time of flight itself. The concentrations of events consisting of $p \bar{p}, \pi^{+} p$, and $\pi^{-} p$ are clearly separated from each other and from the rest of the data, so it is simple to determine the abundances of those event types. The $\pi p$ events come primarily from beam-gas scattering, as is evident from the fact that more protons than antiprotons are observed. $K^{+} K^{-}$events also are fairly well separated from muons and pions, so an estimate of their fraction is possible. $K \pi$ events are possible, though they must alwayo come from interactions from which at least one final-state particle has been

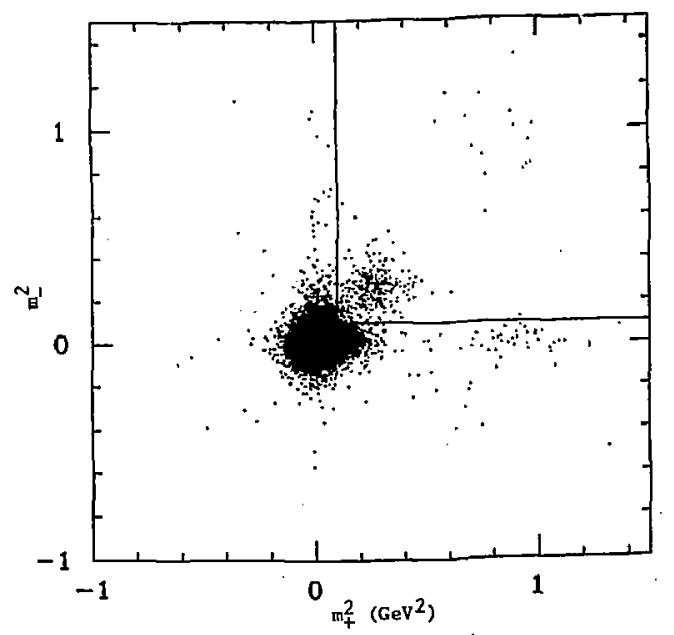

Figure 9.8. Scatter plot of time-of-flight $m_{+}^{2}$ us $m_{-}^{2}$. The abscissae of each point is $\mathrm{m}^{2}$ of the positive track, and the ordinate is $\mathrm{m}^{2}$ of the corresponding negative track. The total number of entries is 37,536 .

missed by the detector. It is not possible to separate them from $\pi^{+} \pi^{-}$and $\mu^{+} \mu^{-}$ data, so their fraction is simply a guess based on what can be seen in Fig. 9.8.

The relative abundances of events types are

$$
\begin{aligned}
f_{\mu \mu} & =0.7000, \quad f_{\pi \pi}=0.2895, \quad f_{\pi K}=0.0005 \\
f_{K K} & =0.0080, \quad f_{p \bar{p}}=0.0005, \text { and } \quad f_{\pi p}=0.0015
\end{aligned}
$$

ising these fractions, the wr'ght for each event type is determined by multiplying the appropriate fraction by the product of the two single-particle weights. To normalize, the six weights are summed, and each weight is divided by that sum. Hence, the sum of all weights for a given event is unity. 

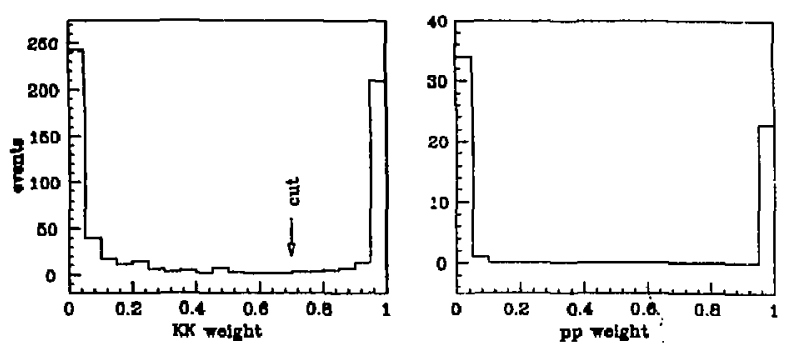

Figure 9.9. The $p \bar{p}$ and $K^{+} K^{-}$event weight for the time-of-fight analygis after the $\chi^{2}$ have been made.

\subsection{Selecting Kaon and Proton pairs}

The firat cut imposed on time-of-flight residuals is to require that for both tracks $m_{i}^{2}>0.01 \mathrm{GeV}^{2}$. It is shown by two intersecting lines on Fig. 9.8. Clearly it does not reject any kaon pairs which could be separated with confidence from the background. The second cut requires that each track have a $\chi_{x}^{2}$, as calculated from Eqn. 8.9, less than 6.0 for kaons and 8.0 for protons. There are two degrees of freedom, so $\chi^{2}=6$ is the $95 \%$ level of the $\chi^{2}$ distribution.

The final cuts are on the event weights. Fjgure 9.9 shows histograms of the event weights for kaon pairs and proton pairs for only those events in which both tracks pars the corresponding $x^{2}$ cut. Keeping only those events with either a $K^{+} K^{-}$weight or a $p \bar{p}$ weight greater than 0.7 results in the final samples of $240 \mathrm{~K}^{+} \mathrm{K}^{-}$events and $23 \mathrm{pp}$ events. A sum over events of the difference of the event weight from unity gives background estimates of five background events in the $K^{+} K^{-}$sample and none is the pip sample. Note that these background estimates include only contributions from time-of-fight misidentification and explicitly assume gaussian distributions for the errors.

\subsection{BACKgRound Estimates}

From the way that the event weights are defined, it is simple to estimate how much background is present from gaussian distributed fuctuations of the large pion-pair and muon-pair samples. The effect of non-gaussian tails, however, is more difficult to estimate. A rough idea can be had from looking at the size of the tail on the negative side of the time residual distribution. An easy way to do that is simply to repeat the analysis and look for particle pairs in which each has, assuming we are studying kaon pairs, a time-of-flight mass squared of $m^{2}=-m_{K}^{2}+m_{\pi}^{2}$. It is reasonable to translate by $m_{\pi}^{2}$ in this way because the expected time residual is approximately proportional to the square of the particle mass. The whole analysis based on $x^{2}$ and event weights follows without change.

One finds that 81 events pass the "kaon-pair" $x^{2}$ cut, but only 4 pass the cut on event weights. These numbers actually are ronsistent with gaussian tails, so we conclude that the background from time-of-flight misidentification in the kaon-pair sample is only the order of 5 to 10 events. The gaussian distributions predict zero background in the proton-pair sample, but 2 events pass the cuts when analyzed for negative $m^{2}$. Thus the corresponding background in the proton-pais sample is about 2 events.

Another potential source of background is from beam-gas collisions. Only 3 kaon-pair events with non-zero charge pass all of the cuts. Also, the distribution of the $z$-component of the event vertex is completely consistent with assuming all observed $K^{+} K^{-}$events to be from beam-beam collisions. For the proton pairs, there are 23 events consisting of two positively charged protons, but there are none with two antiprotons. The events of charge 2 have a vertex distribution which $\vdots_{3}$ consistent with being uniform in $z$, while the charge 0 events are clustered well within the beam-beam region. Since the probability for an incident electron to scatter two protons from a gas molecule is expected to be much giater than the probability for pair production of protons in such a collision, all evidence indicates that none of the observed $p \bar{p}$ events are produced from beam-gas 
collisions. Additional background from beam-beam events in which some particles are not detected must be proportionally less than the corresponding background in the pion-pair sample and is completely negligible with respect to the statistical precision of the $K^{+} K^{-}$and $p \bar{p}$ samples.

\subsection{ANaLYSIS OF THE $K^{+} K^{-}$MASS SPECTRUM}

\subsubsection{Dats Reduction and Normalization}

The $K^{+} K^{-}$data are analyzed in the region $1.3 \leq W_{X K} \leq 2.3 \mathrm{GeV}$. Below the lower cut the trigger inefficiency is too severe to be adequately modeled. Except for the invariant-mass cut and the time-of-flight cuts which have been presented, all other cuts on the data are identical to those used in the pion-pair analysis.

Because of the dependence of the time-of-fight cuts on details of the detector geometry, and because of the greater importance of kaon decays compared with pion decays, all Monte Carlo results include the complete detector simulation. Simulation of the time-of-fight system has been discussed in Section 9.2. The decay products of kaons which have decayed in flight and of positive kaons which have decayed after ranging out in the detector material are included in full detail. The trigger is simulated in two ways. First, the dominant effect at low momentum is caused by electromagnetic range-out and is simulated during the detailed detector simulation by use of Eqn. 9.2. Second, the inefficiency due to nuclear interactions is assumed to be the same as for pions and is parameterized by Eqn. 6.1. While this surely is a crude approximation, the effect is not so important in the kaon-pair analysis because of relatively large statistical errors and because of greater importance of the simple electromagnetic energy loss. The errot estimates on the efficiency are expanded by a factor of 1.5 relative to those for the pion pairs. Figure 9.10 shows a plot of the detection efficiency as calculated by the detector simulation for events generated within the cut $\left|\cos \theta_{1 \mathrm{ab}}\right|<0.65$.

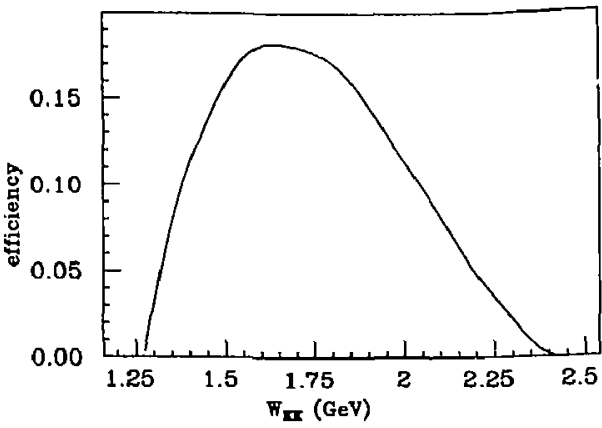

Figure 9.10. The detection efficiency for $K^{+} K^{-}$as calculated by Monte Carlo detector simulation assuming an angular distribution in the $K^{+} K^{-}$ center-of-mass frame of $\sin ^{4} \theta$.

The angular distribution assumed is appropriate for a tensor resonance produced with helicity two.

The normalization again is obtained from the electron-pair data. To avoid a relatively difficult analysis of clectron pairs in data taken with the nitrogen Cerenkov radiator, small angle Bhabha scattcring data from the luminosity monitora is used to extrapolate the effective luminosity measured from data taken with the isobutane radiator. Within a given data-taking season the luminosity monitors remain in the same position relative to the beam, so they are reliable when used to obtain the ratio of Juminosities for two data samples taken within the same year.

The normalization from isobutane data is determined before making any cuts on the lime-of-flight information, and it is measured within a fiducial cut requiring both tracks to pass at least one centimeter inside the edge of a barrel shower counter. Full detector simulation, including EGS for electromagnetic interactions in the beam pipe and drift chamber material and in the material preceding the 
shower counter trigger, is made for Monte Carlo eleetron pairs in order to calculate the effective luminosity corresponding to the number of electron pairs observed in the isobutane data. Note that in this case the effective luminosity is close to the actual beam luminosity, since the detector simulation includes all known inefficiencies. The result from the 1989 isobutane data alone agrees with the result calculated in Section 4.10 for the same data but with different cuts. The total for the combined data set is

$$
\mathcal{L}=169.6 \pm 5.1 \mathrm{pb}^{-1}, \quad \text { (full data set) }
$$

In Fig, 9.11 is a histogram of the $K^{+} K^{-}$invariant mass from date. The spectrum in dominated by a peak centered about $1.525 \mathrm{GeV}$, which is identified as the $f^{\prime}$ resonance. But there also is a relatively large number of events below the $f^{\prime}$ peak, suggesting a significant background under the resonance. The overplotted histogram and smooth curve refer to the model which is presented and fit in the following two sections.

\subsubsection{Model for $\gamma \gamma \rightarrow K^{+} K^{-}$}

The model which is used to fit the data is a coherent sum of Breit-Wigner resonant amplitudes including all three tensor mesons. All resonances are assumed to be produced only with helicity two, as there are not enough data to consider seriougly the angular distribution. The relative phases of the three resonances are taken to be real and positive, in accordance with $S U(3)$ quark model predictions ${ }^{69}$

For the purpose of fitting the data, the cross section for $\gamma \gamma \rightarrow K^{+} K^{-}$is written in this convenient form:

$$
\begin{aligned}
\frac{\mathrm{d} \sigma}{\mathrm{d} \Omega}=\frac{1}{2} R_{f}^{2} & +\frac{1}{2} R_{A_{2}}^{2}+\frac{1}{2} R_{f^{\prime}}^{2}+R_{f} R_{A_{2}} \cos \left(\delta_{f}-\delta_{A_{2}}\right) \\
& +R_{f} R_{f^{\prime}} \cos \left(\delta_{f}-\delta_{f^{\prime}}\right)+R_{A_{2}} R_{f^{\prime}} \cos \left(\delta_{A_{2}}-\delta_{f^{\prime}}\right) .
\end{aligned}
$$

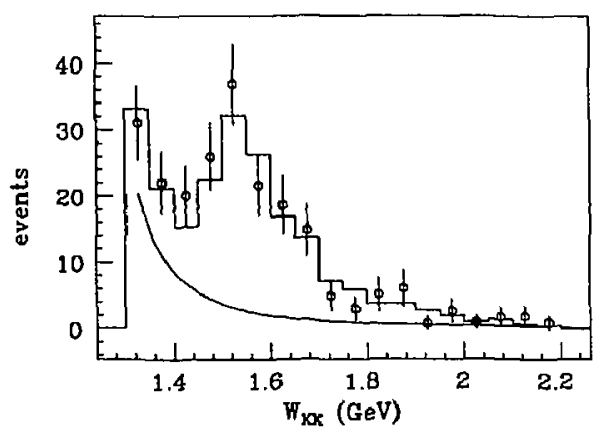

Figure 9.11. The measured $K^{+} K^{-}$invariant-mass spectrum. The points with error bars are data, while the overplotted histogram is the best fit of the model presented in Section 9.7.2. The smooth curve is an estimate of the continuum background, which is included in the histogram as well.

The phase shifts are given by $\tan \delta_{r}=m_{r} \Gamma(s) /\left(m_{r}^{2}-s\right)$, and the resonance terms are

$$
R_{\mathrm{r}}=5 \sqrt{\frac{1}{2}} d_{20}^{2}(\theta) \frac{2}{\sqrt{s}} \frac{m_{\mathrm{r}}\left[\Gamma_{\gamma \gamma}(s) \Gamma_{K} \bar{K}(s)\right]^{1 / 2}}{\left(\left(m_{r}^{2}-s\right)^{2}+m^{2} \Gamma_{r}(s)^{2}\right]^{1 / 2}} .
$$

The factor of $\sqrt{1 / 2}$ comes from the fact that the isospin-zero amplitude is half $K^{+} K^{-}$and half $K^{0} \overline{K^{0}}$. The energy dependencies of the widths are parameterized as in Eqn. 7.32, except that $m_{\pi}$ must be replaced by $m_{K}$ for $\Gamma_{r}(s)$ and $\Gamma_{K \bar{K}}(s)$, and by $m_{\gamma}=0$ for $\Gamma_{\gamma \gamma}(s)$. The properties assumed for the $f$ and $A_{2}$ resonances are given in Table 1.1 , except that the DELCO result, $\mathrm{V}_{f \rightarrow \gamma \gamma}=\mathbf{2 . 7 7} \pm 0.31 \mathrm{keV}$, is used. This is the result obtained when the coupling is assumed to be only in helicity two and the amplitudes are not unitarized. Questions of unitarity are not, considered in the kaon-pair analysis, because the continuum praduction is not undergtood and, anyway, the statistical precision is $t$ sufficient that it matters.

In addition to the $f, A_{2}$, and $f^{\prime}$ there is another $J^{P}=2^{+}$meson called the $\theta(1690)$ in the relevant mass range which has been observed to decay to $K \bar{K}$. 
However, neither its branching ratio to $K \bar{K}$ nor to $\gamma \gamma$ are known. Furthermore, it is in a region which still is dominated by the other three tensor mesons. Certainly one can see no evidence of it in the DELCO data. We do not atternpt to set a hard limit on the product of branching ratios, because any such regult would be highly model dependent due to interference with the long tails of the other resonarces. In Ref. 5 one finds the upper limit $\operatorname{BR}\left(\theta \rightarrow K^{+} K^{-}\right) \cdot \Gamma_{\theta \rightarrow \gamma \gamma}<0.08 \mathrm{keV}$.

Figure 9.12 shows a plot of the resulting cross section with the two-photon luminosity function folded in. The spectrum is integrated numerically over the angular range $-0.6 \leq \cos \theta \leq 0.6$ and assumes a cut on total transverse momentum of $k_{\perp} / W_{\Upsilon \gamma} \leq 0.2$. Compared with that is a plot of the result with the $f$ and $A_{2}$ resonances not included. One can see that the interference has a large effect on the predicted $f^{\prime}$ peak. That raises the issue of how dependent is the spectrum in the $f^{\prime}$ region on the parameterization of the energy-dependent widths of the $f$ and $A_{2}$. In fact, if the widths are given no energy dependence at all, then the cross section in the $f^{\prime}$ region and above is decreased by $25 \%$ or more. However, when the parameter $a$ in Eqn. 7.32 is changed from zero to infinity, the change in the spectrum is negligible with respect to the statistical errors of the data. Thus the results are not too sensitive to the details of the perameterization of the resonance shape, but it is essential to assume some reasonable parameterization.

Some amount of continuum production must also be expected, but there exists no reliable prediction for it in the energy range accessible to DFLCO. The Born term gives a prediction which is much too large to fit the data, as one can see by comparing Fig. 9.11 with Fig. 9.12. There are three reasons why that would be expected. First, the assumption of point coupling generally is worse for kaons than for pions. Second, the $K K$ phase shifts are known to be large even near threshold, so Watson's theorem (Eqn. 7.43) would require substantial modifications of the Born term. Third, inelastic effects are more important than for $f \rightarrow \pi^{+} \pi^{-}$, so even Watson's theorem is not useful. Furthermore, the energy is too low to rely on QCD predictions. Therefore, we follow the procedure of Ref. 7 and introduce

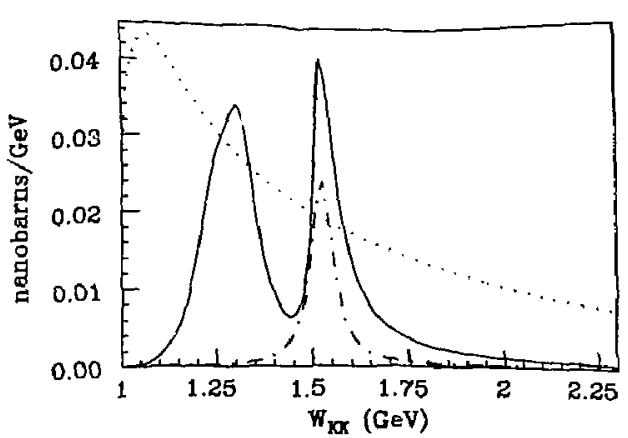

Figure 9.12. Predictions for the $\boldsymbol{K}^{+} \boldsymbol{K}^{+}$mass spectra. The solid curve is a coherent sum of the three tensor meson resonances. The dot-dash curve showe the $f^{\prime}$ contribution alone. The two-photon widths are sssumed to bew production as given by the Born term (Eqn. 7.27 with $m_{\pi}$ replaced by $\left.m_{K}\right)$.

arbitrary functions of $W_{K K}$ to describe the background for the purpose of fitting the two-photon width of the $f^{\prime}$. The functions used are of the forms

$$
B_{1}(W)=A \cdot \exp \left(-\frac{(W-\bar{W})^{2}}{2 \sigma^{2}}\right), \quad \bar{W}<1.1 \mathrm{GeV}
$$

and

$$
B_{2}(W)=A \cdot\left[W-W_{0}\right]^{-n}, \quad 1 \leq n \leq 10 .
$$

The parameters $A, W_{0}$, and $\sigma$ are allowed to vary freely, and several fixed values are tried for $n$ and $\bar{W}$. Both forms give good fits to the data, whereas the resonance terms alonc fail to describe the spectrum below the $f^{\prime}$ peak. Also, both forms have a reasonable shape in the resonance region, since one generally expects the continuum spectrum to be continuously decreasing with increasing energy. Note that QCD predicts that at suffieiently high energy the spectrum should fall 
as $1 / W^{7}$ when convoluted with the EPA spectrum, while the Born prediction decreases as $1 / W^{3}$. Probably, the truth in the $f^{\prime}$ energy range is somewhere between those two extremes.

Events generated by this model are processed through detector simulation and the full analysis procedure. Because of the limited statistical precision of the data, it is not necessary to vary the resonance masses and full widths in the fit. It is important to be able to vary the two-photon widths of all three resonances. That is simple to do with the cross section expressed as it is in Eqn. 9.16. One simply retains throughout the analysis the fraction of the total event weight contributed by each of the six terms. Each term is proportional either to one $\gamma \gamma$ width or else to the square root of the product of two $\gamma \gamma$ widths, so the fitting program can vary all three $\gamma \gamma$ widths independently by appropriatily varying the contributions of the six terms to the sum.

\subsubsection{Fitting the Invariant-Mass Spectrum}

There are a total of nine parameters which may be varied within a single run of the fitting program:
1. $\operatorname{BR}\left(f^{\prime} \rightarrow K \bar{K}\right) \cdot \Gamma_{f^{\prime} \rightarrow 7 \gamma}$
2. $\operatorname{BR}\left(A_{2} \rightarrow K \bar{K}\right) \cdot \Gamma_{A_{2} \rightarrow \gamma \gamma}$
3. $\mathrm{BR}(f \rightarrow K \bar{K}) \cdot \Gamma_{f \rightarrow \gamma \gamma}$

$$
\begin{aligned}
& =0.040 \pm 0.013 \mathrm{keV} \\
& =0.080 \pm 0.011 \mathrm{keV} \\
\mathcal{L} & =1699.6 \pm 5.1 \mathrm{pb}^{-1}
\end{aligned}
$$
4. Luminosity.
5. Norm. of ToF background.
$N_{\text {tof }}=1,0 \pm 1.0$
6. Efficiency error at $W=1.3 \mathrm{GeV}$.

$$
\varepsilon_{1}=0: t 0.14
$$
7. Efriciency error at $W=2.3 \mathrm{GeV}$.
$\epsilon_{2}=0: \pm 0.10$
8. Background parameter \#1.
9. Background parameter \#2.

Parameter number 5 refers to the background estimate which follows from the gaussian event weights. That background is only a few events total and is confined mostly to the invariant mass range above the $f^{\prime}$ region. This parameter allows the normalization of that background to vary within reasonable limits and is not important. Parameters 6 and 7 account for the uncertainty in the trigger efficiency, which is assumed to vary linearly between the values, $\varepsilon_{1}$ and $\varepsilon_{2}$, specified at the upper and lower limits of the invariant mass range. The last two parameters are those of the background parameterizations specified in equations 9.18 and 9.19 .

As a specific example, consider a fit in which the background parameterization of Eqn. 9.19 with $n=3$ is assumed and all nine parameters are allowed to vary within the specified limits. Table 9.1 shows the explicit results of the fit, and Fig. 9.11 shows a comparison of the result with the data. A large part of the contribution to $\chi^{2}$ comes from the single low bin at $W=1.925 \mathrm{GeV}$. When only the first 10 bina are included in the fit, then $\chi^{2}=9.8$, and the fitted $f^{\prime}$ two-photon width decreases by $0.003 \mathrm{keV}$.

Such a fit as is shown in Table 9.1 assumes gaussian distributions for those parameters which os constrained within known error limits. The resulting overall cror estimate for the $f^{\prime}$ t... 2 photon width corresponds to addition of the various erfor contributions in quadrature. I.et us consider the contributions to the error individually. When only the $f^{\prime}$ two-photon width and the background parameterization (with $n=3$ ) are allowed to vary, ther the best fit for the two-photon width does not change, but the error estimate becomes only $\pm 0.017 \mathrm{keV}$. Fixing the background as well yieids an estimate of the statistical error of $\pm 0.015 \mathrm{keV}$. Contributions to the error from the other parameters are determined by fixing them one by one at their upper and lower limits and then fitting the spectrum by varying all remaining parameters. Table 9.2 lists the individual contributions. That from the background parameterization takes into consideration the varying results obtained from using Eqn. 9.18 as well as Eqn. 9.19 and also the effects of changing the exponent $n$ between 1 and 10 and the mean of the gaussian between $0.1 \mathrm{GeV}$ and $1.1 \mathrm{GeV}$. Hence the error is larger than in Table 9.1, where $n$ is held fixed at $n=3$. With all things considered, the final 
Table 9.1. A fit of the model for $\gamma \gamma \rightarrow K^{+} K^{-}$to time-of-flight identified data. The parameterization of the background is given by Eqn. 9.19 with $n=3$.

\begin{tabular}{|c|c|c|c|c|c|c|c|c|}
\hline \multicolumn{4}{|c|}{ Parameter } & \multicolumn{2}{|c|}{ Fit Value } & \multirow{2}{*}{$\begin{array}{l}\text { Lower Error } \\
-0.021\end{array}$} & \multicolumn{2}{|c|}{ Upper Error } \\
\hline \multicolumn{4}{|c|}{ 1. $\operatorname{BR}\left(f^{\prime} \rightarrow K \bar{K}\right) \cdot \Gamma_{f^{\prime} \rightarrow \gamma \gamma}$} & 0.070 & $\mathbf{k e V}$ & & \multicolumn{2}{|c|}{+0.025} \\
\hline \multicolumn{4}{|c|}{ 2. $\operatorname{BR}\left(A_{2} \rightarrow K \bar{K}\right) \cdot \Gamma_{A_{2} \rightarrow \gamma \gamma}$} & 0.039 & $\mathbf{k e V}$ & -0.013 & \multicolumn{2}{|c|}{+0.013} \\
\hline \multicolumn{4}{|c|}{ 3. $\mathrm{BR}(f \rightarrow K \bar{K}) \cdot \Gamma_{f \rightarrow \gamma \gamma}$} & 0.080 & $\mathbf{k e V}$ & -0.011 & \multicolumn{2}{|c|}{+0.011} \\
\hline \multicolumn{4}{|c|}{ 4. luminosity } & 169.5 & $p b^{-1}$ & $-5,1$ & \multicolumn{2}{|c|}{+5.1} \\
\hline \multicolumn{4}{|c|}{ 5. ToF background } & \multicolumn{2}{|l|}{1.4} & -1.0 & \multicolumn{2}{|c|}{+1.0} \\
\hline \multicolumn{4}{|c|}{ 6. $E_{1}$} & \multicolumn{2}{|l|}{-0.03} & -0.14 & \multicolumn{2}{|c|}{+0.14} \\
\hline \multicolumn{4}{|c|}{ 6. $\varepsilon_{2}$} & \multicolumn{2}{|l|}{-0.03} & -0.10 & \multicolumn{2}{|c|}{+0.10} \\
\hline \multicolumn{4}{|c|}{ 8. $A$ (Eqn. 8.19$)$} & \multicolumn{2}{|l|}{0.31} & -0.22 & \multicolumn{2}{|c|}{+0.49} \\
\hline \multicolumn{4}{|c|}{ 9. $W_{0}$ (Eqn. 9.19) } & \multicolumn{2}{|l|}{1.09} & -0.10 & \multicolumn{2}{|c|}{+0.08} \\
\hline \multicolumn{9}{|c|}{ Covariance Matrix Correlation Coefficients } \\
\hline \multirow[b]{2}{*}{2} & 1 & 2 & 3 & 4 & 5 & 6 & \multirow[t]{7}{*}{7} & \multirow[t]{8}{*}{8} \\
\hline & -0.201 & & & & & & & \\
\hline 3 & -0.102 & 0.024 & & & & & & \\
\hline 4 & -0.140 & 0.017 & -0.048 & & & & & \\
\hline 5 & -0.012 & -0.013 & -0.006 & -0.013 & & & & \\
\hline 6 & -0.531 & -0.004 & 0.034 & 0.030 & 0.004 & & & \\
\hline 7 & -0.155 & -0.013 & -0.079 & 0.005 & -0.019 & 0.053 & & \\
\hline 8 & -0.280 & -0.234 & -0.131 & -0.054 & $-0,080$ & -0.132 & -0.065 & \\
\hline 9 & 0.330 & 0.139 & 0.090 & 0.035 & 0.080 & 0.057 & 0.062 & -0.972 \\
\hline \multicolumn{4}{|c|}{$\begin{array}{l}\text { Fit range: } \\
x^{2}=18.1\end{array}$} & \multicolumn{2}{|c|}{14 bins } & & & \\
\hline
\end{tabular}

result for the $f^{\prime}$ is obtained by adding the systematic errors in quadrature:

$$
\Gamma_{f^{\prime} \rightarrow \gamma \gamma} \cdot \operatorname{BR}\left(f^{\prime} \rightarrow K \bar{K}\right)=0.07 \pm 0.04 \mathrm{keV}
$$

Unfortunately, the branching ratio of the $f^{\prime}$ to $\bar{K} \bar{K}$ is not yet known to sufficient acclirac: to quote a measurement of the two-photon width alone.

Table 9.2- Contributions to the error on $\operatorname{BR}\left(f^{\prime} \rightarrow K \bar{K}\right) \cdot \Gamma_{f^{\prime} \rightarrow \gamma \gamma}$ from the fit to the $K^{+} K^{-}$invariant-mass spectrum.

\begin{tabular}{|c|c|}
\hline Source of Error & Error in keV \\
\hline gize of $K^{+} K^{-}$sample & \pm 0.015 (stat.) \\
\hline luminosity measurement & \pm 0.002 (syst.) \\
\hline detection efficiency & \pm 0.015 (syst.) \\
\hline $\begin{array}{c}\mathrm{BR}(f \rightarrow K \bar{K}) \cdot \Gamma_{f \rightarrow \gamma \gamma} \text { and } \\
\operatorname{BR}\left(A_{2} \rightarrow K \bar{K}\right) \cdot \Gamma_{A_{2} \rightarrow \gamma \gamma}\end{array}$ & \pm 0.015 (syst.) \\
\hline background parameterization & \pm 0.027 (syst.) \\
\hline
\end{tabular}

\subsection{MEASUREMENT OF $\gamma \gamma \rightarrow p \bar{p}$}

Not enough proton pairs have been detected to allow any analysis of the shapes of kinematic distributions. Therefore, se consider only a measurement of the average cross section for $\gamma \gamma \rightarrow p \bar{p}$ over the range in which data are available $(2.2<W<2.9 \mathrm{GeV})$.

There is no large sample of protons in any data set which may be used to measure the detector response for protons, so the Monte Carlo detector simulation must be relied upon to calculate the detection efficiency for $p \bar{p}$. The dominant systematic effect again comes from the barrel shower counter latch efficiency. The contribution from electromagnetic range-out is calculated the same as for 
the $K^{+} K^{-}$analysis, using Eqn. 9.2. For protons, the resulting momentum cutoff is predicted to be at about $0.6 \mathrm{GeV}$. Above that momentum, some inefficiency is caused by nuclear interactions. An HETC calculation predicts that the shower counter latch efficiency for protons incident on a counter is $92 \%$ at $p=0.7 \mathrm{GeV}$ and rises slowly to $98 \%$ at $p=2.0 \mathrm{GeV}$. The HETC program does not handle antiprotons, so the same efficiency is assumed for them. Thus the loss of events from nuclear interactions is estimated to be about $15 \%$ overall. The error in thi result can only be guessed, but even a very conservative estimate gives an error bar no larger than the statistical error.

To measure the detection efficiency, Monte Carlo events of the type $e^{+} e^{-} \rightarrow$ $e^{+} e^{-} p \bar{p}$ are generated using the EPA luminosity function and a unit crose section for $\sigma_{\eta \eta p}$. Thus the Monte Carlo asaumes a uniform :ingular distrihutins. The detector is simulated for the generated events, and the full analysis procedure is applied, just wo for data. One finds that the predicted detection efficiency rises sharply from zero at $W=2.2 \mathrm{GeV}$ and peaks at absut $2.7 \mathrm{GeV}$, falling slowly sbove that point. The maximum efficiency ' about $5 \%$.

The luminosity of the data is $170 \mathrm{pb}^{-1}$, and $23 \mathrm{p} \bar{p}$ events are found within the range $2.2<W<2.9 \mathrm{GeV}$. Assuming a uniform angular distribution to calculate the detection efficiency, this corresponds to a cross section for $\gamma \boldsymbol{\gamma} \rightarrow p \bar{p}$, integrated over the acceptance $\left|\cos \theta_{\mathrm{cmu}}\right|<0.6$, of $1.01 \pm 0.21 \mathrm{nb}$. The observed angular distribution may be taken into account by calculating with Monte Carlo the detection efficiency as a function of both $W$ and $\cos \theta_{\text {cms }}$. When such a calculation is used to correct the data event by event, then the result is a cross section of $1.25 \pm 0.32 \mathrm{nb}$. The errors quoted are statistical only. Including a $20 \%$ uncertainty in the detection efficiency and accounting for the dependence on the sssumed angular distribution, we arrive at the result

$$
\sigma_{\tau \gamma \rightarrow p \bar{p}}=1.2 \pm 0.5 \mathrm{nb} \quad\left|\cos \theta_{c \mathrm{mB}}\right|<0.6,
$$

averaged over the region $2.2<W<2.9 \mathrm{GeV}$
10. Conclugione

\subsection{The QED Channel}

Kinematic distributions from s sample of over $4 \mathbf{j}, 000$ events from the process $e^{+} e^{-} \rightarrow e^{+} e^{-} e^{+} e^{-}$have been compared with leading-order QED predictions. The shapes of the distributions for untagged $\left(Q^{2} \approx 0\right)$ data are in complete agreement with leading-order $\left(\alpha^{4}\right)$ QED predietions within statistical error limits which range over the histogram bins from $1 \%$ at $W_{\min }=0.6 \mathrm{GeV}$ to $10 \%$ at $W_{\max }=2.6 \mathrm{GeV}$. Figures 4.14 and 4.16 show comparisons between data and QED predictions for the invariant mass and angular distributions of the detected electron pair. The shepes of distributions from tagged data $\left(\overline{Q^{2}}=0.4 \mathrm{GeV}^{2}\right)$ agree as well, with statistical errors ranging from $4 \%$ to $22 \%$. Figures 4.19 and 4.20 show those results. Other experiments also have published resulto on this reaction channel, but only with far less statistical precision and only for relatively $\operatorname{large}^{70}(W>1 \mathrm{GeV})$ or amall ${ }^{\mathbf{7 1}}$ $(W<0.5 \mathrm{GeV})$ invariant mnases.

Absolute normalization of the measured eross seetions has been obtained from the beam luminosity as mesoured from another QED process, $e^{+} e^{-} \rightarrow \mu^{+} \mu^{-}$, but only within systematic errorn of 7 w 10 percent. At that level the cross sections agree with QED predictions. The measurement of the four-electron final stale is itself used to detersaine the normalization of the $E^{+} e^{-} \mu^{+} \mu^{-}$channel and the two-photon hadronic channels to an accuracy of better than $3 \%$.

\subsection{Cros,s SECTION FOR $\gamma \gamma \rightarrow \pi^{+} \pi^{-}$}

10.2.1 Measurements from Untagged Data $\left(Q^{2} \approx 0\right)$

The cross section for $\gamma \gamma \rightarrow \pi^{+} \pi^{-}$has been measured at $Q^{2} \approx 0$ from a sampls of 21,000 pion pairs plus 50,000 muon pairs within the laboratary acceptance $0.6<W<2.0 \mathrm{GeV},|\cos \theta|<0.6$. DELCO has an advantage over previous measurements due to the fact that electron pairs need not be subtracted from the data and that the normalization for subtraction of muons pairs can be 
obtained with an accuracy of $2.3 \%$ from a measurement of the four-electron final atate. The normalization of the pion-pair cross section is normalized to the same QED measurement, but uncertainty in th: detection efficiency, due to nuclear interactions, contributes systematic errors of 5 to 10 percent.

Figure 10.1 shows the differential cross section measured for the invariant mass of the pion pair, with all known detector effects, including the nonientum resolution, corrected for by the procedure detailed in Appendix C. The prominent peak is due to production of the $f(1270)$ resonance. Figure 10.2 shows the measured angular distribution of the pions in their center-of-mass system. It has been corrected for detector effects, of which the most important by far is the loss of acceptance caused by the boost of the $y \gamma$ ayatem in the laboratory frame of reference. The data are compared with predictions for helicity-two and helicityzero coupling of $\gamma \gamma$ to the $f$ resonance. It is cleas that the helicity-two assumption is preferred. A detailed simultareous fit to both distributions, assuming that the continuum is described by the simple Born term prediction, gives a limit on the ratio of the helicity-zero to helicity-two ry coupling (see Eqn. 8.11):

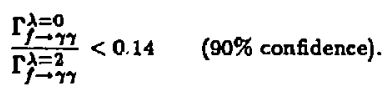

This is consistent with theoretical expectations and may be compared with the best previously published limit of $0.12 \pm 0.39 .^{\circ}$ The latter result was obtained from $\gamma \gamma \rightarrow \pi^{0} \pi^{0}$ and is somewhat less model dependent, because of the lack of a significant continuum contribution to the neutral channel.

In Chapter 2 we have seen how the cross section for $e^{+} e^{-} \rightarrow e^{+} e^{-} \pi^{+} \pi^{-}$ may be decomposed for untagged events into a luminosity function for quasjreal photon pairs times the cross section $\sigma_{\gamma \gamma \rightarrow \pi^{+} \pi^{-}}$. By using the equivalentphoton approximation (EPA), the luminosity function is easily calculated as a function of $W_{\boldsymbol{\gamma} \gamma}$. Therefore, one can determine $\sigma_{\boldsymbol{\gamma \gamma} \rightarrow \pi^{+} \boldsymbol{\pi}^{-}}$from the data of

† The recent results of the PEP. $1 /$ PEP-9 experiment ${ }^{6}$ heve roughly the asme advantages.

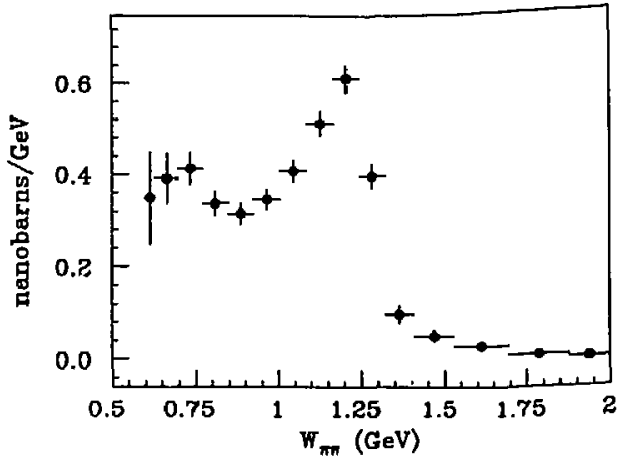

Figure 10.1. The measured differentia! cross section for the invariant mass of pion pairs from $e^{+} e^{-} \rightarrow e^{+} e^{-} \pi^{+} \pi^{-}$. The data have been corrected for all known detector effects within the acceptance defined by $\left|\cos \theta_{1 \leq b}\right|<0.6, k_{\perp} / W<0.2$, and $k_{\perp}<0.3 \mathrm{GeV}$

Fig. 10.1. First, the data are corrected for the loss of acceptance due to the boost of the $\gamma \gamma$ system in the $\pm z$ direction. That is done by using the EPA Monte Carlo to predict the distribution of the boost and then calculating the inefficiency from the cut $\left|\cos \theta_{\text {lab }}\right|<0.6$. The angular distribution of the reanance decay is assumed to be $\sin ^{4} \theta$, as is appropriate for helicity-two production. Then the result is divided by the calculated luminosity function. Figure 10.3 shows the result. It is not extrapolated to an acceptance of $4 \pi$ steradians, wo when comparing with a theoretical model, the cross section must be integrated over the range $-0.6<\cos \theta_{\mathrm{cm}}<0.6$. Note that there is a small amount of model dependence in the result, because in order to correct for the effects of the detector acceptance, some assumption must be made about the angular distribution. It is assumed to be given by a coherent sum of the Born term and the $f$ resonance in helicity two, which has been shown to give an excellent fit to the observed angular distribution. The angular distribution cannot be observed by DELCO outside of 


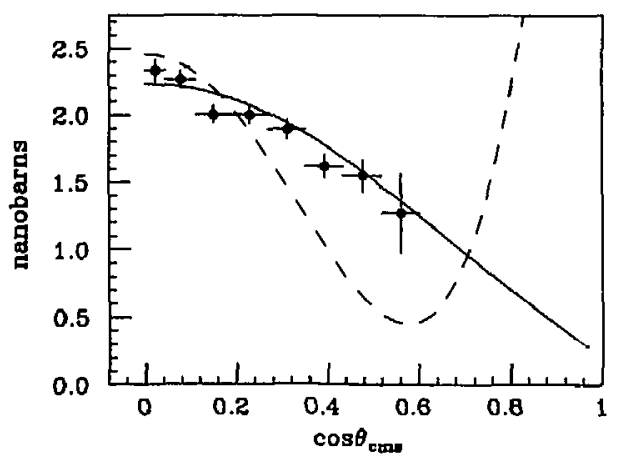

Figure 10.2. The measured differential crose section for $\cos \theta_{\mathrm{cm}}$ of pion pairs from $e^{+} e^{-} \rightarrow e^{+} e^{-} \pi^{+} \pi^{-}$. The smooth curves show predictions for helieity-two (solid curve) and helicity-zero (dashed curve) coupling of $\gamma \gamma$ to the $f$ resonance. The data have been corrected for all known detector effects within the acceptance defined by $1.0<W<1.5 \mathrm{GeV}$, $k_{\perp} / W<0.2$, and $k_{\perp}<0.3 \mathrm{GeV}$.

$|\cos \theta|<0.6$, so to extrapolate to the full solid angle would be even more model dependent.

The smooth curve shown in Fig. 10.3a represents a calculation of the unitarized Lyth model (Eqn. B.1) for $\sigma_{y \gamma \rightarrow \pi^{+}}$, integrated over the range $-0.6<\cos \theta_{\mathrm{cms}}<0.6$ with $\Gamma_{f \rightarrow \gamma \gamma}^{\lambda=0}=0$ and $\Gamma_{f \rightarrow \gamma \gamma}=3.34 \mathrm{keV}$ (from Eqn. 8.8). There appears to be substantial disagreement both below and above the $f$ peak. However, systematic errors are not shown in Fig. 10.3. The uncertainty in the beam luminosity and in the $\pi^{+} \pi^{-}$trigger efficiency is larger than the disagreement below the $f$ peak. Also, there is some uncertainty in the energy scale of the experiment, and the agreement is better if the $f$ mass is shifted downward by about $1 \%$. The digagreement just above the $f$ peak is not understood. Figures 8.1 and 8.2 show how well the measured spectra agree with the model prediction
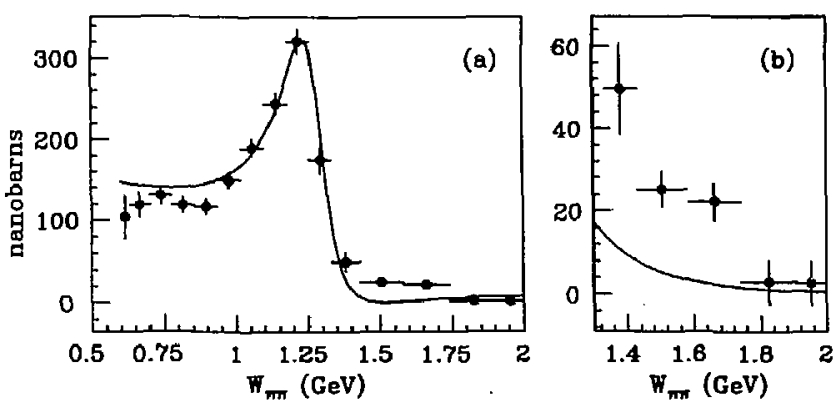

Figure 10.3. The cross section for $\gamma \gamma \rightarrow \pi^{+} \pi^{-}$measured from untagged data for $-0.6<\cos \theta_{\mathrm{cm}}<0.6$. The error bars include only atatistical errors. The smooth curve in (a) is the theoretical prediction of Eqn. 8.1 assuming purely helicity-two coupling to the resonance. The smooth curve in (b) is the QCD prediction for continuum production given by Eqn. 7.2.

when systematic errors are taken into account. For reference, in Fig. 10.3b the QCD prediction of Eqn. 7.2 is compared with the data points between $1.4 \mathrm{GeV}$ and $2.0 \mathrm{GeV}$.

The two-photon width of the $f$ has been determined from the data by fitting the observed mass spectrum with the tieoretical model. With systematic errors accounted for, good fits are obtained up to $w=1.4 \mathrm{GeV}$. There is significant disagreement above that point, but it has been found to contribute only a minor amount of uncertainty to the measured two-photon width. Resulto have been presented in Chapter 8 for several different theoretical assumptions about the angular distribution and unitarity constraints. In order to compare with previous measurements, first the results are presented under theoretical assumptions common to most of the measurements. Most experiments have assumed a simple model of a coherent sum of the Born term (Eqn. 7.27) and a Breit-Wigner amplitude (Eqn. 7.34) with no corrections made to satisly unitarity 
constraints. The coupling of $\gamma \gamma$ to the $f$ is assumed to occur only in the helicitytwo amplitude. With those assumptions, the DELCO result is, after extrapolating to $Q^{2}=0$ by the GVDM form factor (see Eqn. 8.7 and Section 8.3 .5 ),

$$
\Gamma_{\gamma \gamma}=2.81 \pm 0.31 \mathrm{keV}, \quad(\lambda=2, \text { no unitarization }) .
$$

This is compared in Table 10.1 with previously published measurements. All of the measurements quoted assume that the coupling is exclusively helicitytwo. The CELLO result assumes the Mennessier model (see Section 7.5), which satisfies unitarity constraints which are essentialiy elastic except for a small $K \bar{K}$ contribution. However, as stated by the CELLO collaboration in Ref. 61, the unitarity constraint has negligible effect. None of Mennessier's options for vector meson exchanges and scalar resonances are used in the CELLO analysis, so their model is not significantly different from the simple Born term plus Breit-Wigner. The Mark II and PEP-4/PEP-9 results allow the normalization of the teris describing the interference of the resonance and continuum to float free in the fit, and the Mark II analysis of $P E P$ data assumes that the continuium is described by a QCD prediction (see Equ. 7.2) above $W=1 \mathrm{GEV}$. However, these differing assumptions do not affect the results for the two-photon width significantly with respect to the stated error limits. The Crystal Ball result is unique in that it is from a measurement of $e^{+} e^{-} \rightarrow e^{+} e^{-} \pi^{0} \pi^{0}$, instead of the usual charged pion channel. The neutral channel has negligible continuum background, so the Crystal Ball result should be relatively model independent.

When corrections are made to the simple model of the Born term plus a BreitWigner amplitude in order to satisfy elastic unitarity constraints according to the prescription by Lyth (see Section 7.4.6), we find that the fitted two-photon width increases by $20 \%$ over the value quoted in Eqn. 10.2 (see Eqn. 8.8). When, in addition, the contribution from helicity-zero coupling is allowed to be free in the fit and both the invariant mass and angular distributions are fit simultaneously,
Table 10.1- Summary of published measurements of $\Gamma_{f \rightarrow \gamma \gamma}$ in chronological order. Ail measurements assume helicity-two coupling. The DELCO result is without unitarity corrections. For each measurement quoted, the first error is statiscical and the second is systematic.

\begin{tabular}{|l|c|c|c|}
\hline Collaboration & Reference & $\left.\Gamma_{f \rightarrow \gamma \gamma} \mid \mathrm{keV}\right]$ & Final State \\
\hline PLUTO, 1982 analysis & 72 & $2.3 \pm 0.5 \pm 0.3$ & $\pi^{+} \pi^{-}$ \\
TASSO & 73 & $3.2 \pm 0.2 \pm 0.7$ & $\pi^{+} \pi^{-}$ \\
Mark II, SPEAR data & 41 & $3.6 \pm 0.3 \pm 0.5$ & $\pi^{+} \pi^{-}$ \\
Crystal Ball & 9 & $2.7 \pm 0.2 \pm 0.6$ & $\pi^{0} \pi^{0}$ \\
Mark II, PEP data & 3 & $2.52 \pm 0.13 \pm 0.38$ & $\pi^{+} \pi^{-}$ \\
CELLO & 61 & $2.5 \pm 0.1 \pm 0.5$ & $\pi^{+} \pi^{-}$ \\
PLUTO, 1984 analysis & 74 & $3.25 \pm 0.25 \pm 0.50$ & $\pi^{+} \pi^{-}$ \\
PEP-4/PEP-g & 5 & $3.2 \pm 0.1 \pm 0.4$ & $\pi^{+} \pi^{-}$ \\
DELCO, this thesis & & $2.81 \pm 0.07 \pm 0.30$ & $\pi^{+} \pi^{-}$ \\
\hline
\end{tabular}

then the result is (from Eqn. 8.11, but extrapolited to $Q^{2}=0$ )

$$
\Gamma_{f \rightarrow \gamma \gamma}=\Gamma_{\gamma \gamma}^{0}+\Gamma_{\gamma \gamma}^{2}=3.47 \pm 0.37 \mathrm{keV} .
$$

The statistical contribution to the crror is only $\pm 0.07 \mathrm{keV}$ and is included in yuadrature with 'lie gystematic er ror. The systematic error includes contributions from the unccrtainty in the ratio of helicity amplitudes and in the degree to which elastic unitarity should be satisfied (see Section 7.4.7), but it is dominated by the uncertainty in the pion-pair trigger efficiency. The result in Eqn. 10.3 is best compared with the Crystal Ball result with no constraint on the ratio of helicity amplitudes. ${ }^{9}$ Their result of $\Gamma_{\gamma \gamma}=2.9_{-0.4}^{+0.6} \pm 0.6 \mathrm{keV}$ is in agreement with DELCO. However, it is unfortunate that the error estimates are so isrge, because this result gives no information on the consistency of the unitarization procedure applied to the model for charged pion-pair production (see Seciion 7.6). When unitarization corrections are not made, the result for $\Gamma_{\gamma \gamma}$ is lower $t$ nan that presented in Eqn. 8.11 by a factor of 0.83 . 


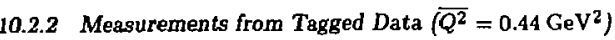

The tagged data have been analyzed according to the same theoretical model as used for untagged data, except that the prediction for the continuum is multiplied by a GVDM form factor $F\left(Q^{2}\right)$ according to Eqn. 2.20. The $Q^{2}$ dependence of the single-tag luminosity function is given by Eqn. 2.18 , and the range of $Q^{2}$ is limited by the geometric extent of the DELCO luminosity counters. The average over the acceptance is $\overline{Q^{2}}=0.44 \mathrm{GeV}^{2}$. This model is found to give an excellent fit to the data within statistical error limits.

For tagged events, the incoming two-photon state consists of all three possible helicity states, and the theoretical expectations for $\sigma_{\gamma \gamma \rightarrow \pi \pi}\left(Q^{2}\right)$ are that the helicity-zero and helicity-one contributions increase rapidly with $Q^{2}$. The measured angular distribution of pion pairs constrains the helicity-one contribution to less than $15 \%$ of the two-photon width but allows the helicity-zero contribution to be as large as or larger than the helicity-two contribution. In Section 8.4 one may find the results of fitting the two-photon width under two different assumptions about the angular distribution. In order to account fully for the uncertainty in the angular distribution, it is best to use the result with the ratio of helicity amplitudes left free in the fit:

$$
\Gamma_{\Upsilon 7}\left(\overline{Q^{2}}=0.44 \mathrm{GeV}^{2}\right)=1.42 \pm 0.33 \mathrm{keV}, \quad \text { (no unitarization) }
$$

The result with the ratios of helicity amplitudes constrained to certain theoretical expectations (see Eqn. 8.14) is consistent with Eqn. 10.4, but the error bar is, of course, much smaller. Figure $\mathbf{1 0 . 4}$ shows a comparison of the tagged measurement with the untagged measurement. Both values assume the theoretical model without unitarity corrections. The decrease in cross section with $Q^{2}$ is consistent with the prediction of the GVDM form factor.

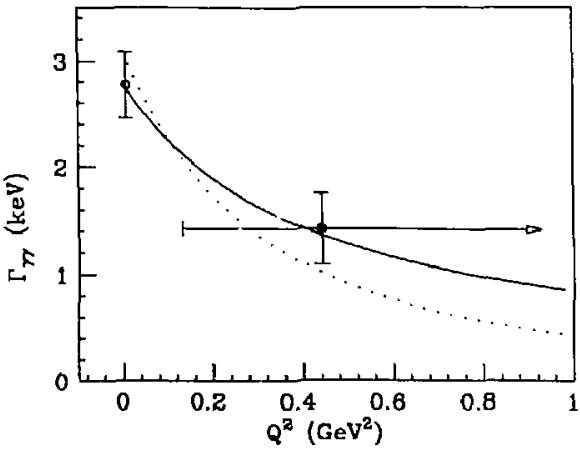

Figure 10.4. The measured $Q^{2}$ dependence of $\Gamma_{f \rightarrow \gamma \gamma}$ compared with the GVDM form factor (solid curve). The horizontal error bar indicates the large range of $Q^{2}$ aver which the tagged measurement is made. The pcint are placed at the average value of $Q^{2}$ predicted by the appropriate $\gamma \gamma$ luminosity function. The dotted curve shows for comparison a simple $\rho$-pole form factor.

\subsection{MEasurement of The $f \prime$ Two. Photon Width}

A sample of 240 events from the process $e^{+} e^{-} \rightarrow e^{+} e^{-} K^{+} K^{-}$have been identified by time-of-flight measurements in the kaon-pair mass range from $1.3 \mathrm{GeV}$ to $2.3 \mathrm{GeV}$. The mass spectrum may be found in Fig. G.11. One can see clear evidence for production of the $f^{\prime}$ resonance. There also is a relatively high cross section below the $f^{\prime}$ peak. The spectrum has been fit to a coherent sum of three interfering tensor resonances, but it still is necessary to assume a significant amount of continuum production. Lacking a reliable theoretical description of the continuum production, the simple parameterizations given by equations 9.18 and 9.19 arc assumed. The branching ratio of the $f^{\prime}$ to $K \bar{K}$ is not known, so the result is expressed as (from Eqn. 9.20)

$$
\Gamma_{f^{\prime \prime} \rightarrow \gamma \gamma} \cdot \operatorname{BR}\left(f^{\prime} \rightarrow K \bar{K}\right)=0.07 \pm 0.04 \mathrm{keV}
$$




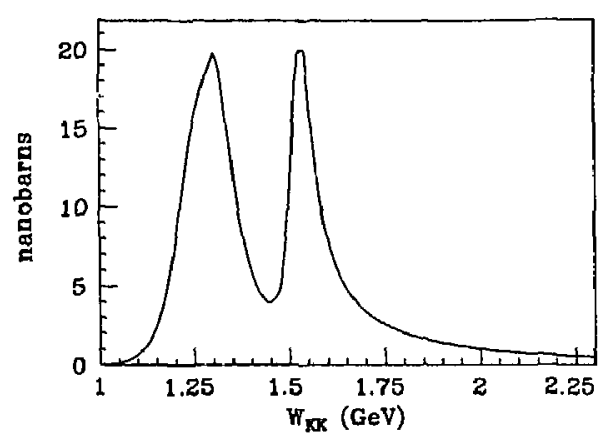

Figure 10.5. Predictions for the "resonant contribution to $\sigma_{77-K+K-}$ integrated over the center-of-mass acceptance defined by $-0.6<\cos \theta$ 0.6. The values used for $\Gamma_{f \rightarrow 7 \gamma}$ and $\Gamma_{f \prime \rightarrow \gamma \gamma}$ are the DELCO measure ments, while $\Gamma_{A_{2} \rightarrow \gamma \gamma}$ is taken from Ref. 6 .

The statistical error is $0.015 \mathrm{keV}$, and the principle contribution to the systematic ersor is from the uncertainty in the shape of the continuum spectrum. Figure 10.5 shows the resonant contribution to the cross section assumed in the fit, including the fitted value of the $f^{\prime}$ two-photon width. The resonances all are assumed to be produced purely with helicity two.

Table 10.2 compares the DELCO result with previous measurements. The DELCO spectrum has been analyzed in the same way as that of TASSO, ${ }^{7}$ вo some of the systematic effects should be the same for the two experiments. Therefore, there may be some discrepancy between TASSO and DELCO. The PEP-1/PEP-9 spectrum is analyzed without including interference between the three tensor resonances. If the same is done for the DELCO spectrum, then the data fit just as well as with interference included (assuming the same background shape), while the fitted two-photon width for the $f^{\prime}$ is increased by a factor of 1.8 up to $0.13 \mathrm{keV}$.

The ratio of the DELCO measurements for the two-photon widths of the $f$
Table 10.2. Comparison of measurements and limits on $\Gamma_{f^{\prime} \rightarrow \gamma \gamma}$ between several experiments. For each measurement quoted, the first error is statistical and the second is systematic.

\begin{tabular}{|l|c|c|}
\hline Collaboration & Reference & $\Gamma_{f^{\prime} \rightarrow \gamma \gamma} \cdot \mathrm{BR}\left(f^{\prime} \rightarrow K \bar{K}\right)[\mathrm{keV}]$ \\
\hline Mark II, SPEAR data & 75 & $<0.6$ \\
TASSO & 7 & $0.11 \pm 0.02 \pm 0.04$ \\
$P E P-\{/ P E P-9$ & 5 & $0.12 \pm 0.06 \pm 0.06$ \\
DELCO, this thesis & & $0.070 \pm 0.015 \pm 0035$ \\
\hline
\end{tabular}

and $f^{\prime}$ may be compared with the $S U(3)$ predictions expressed by Eqn. 1.10. To do so, the branching ratio for the $f^{\prime}$ decaying to $K \bar{K}$ is issumed to be greater than 0.5, as is consistent with the estimate $\Gamma_{f^{\prime \prime} \rightarrow K \bar{K}}=55 \pm 22 \mathrm{MeV}$ from Ref. 76 . Since the quark model does not consider any consequences of the finite width of the resonances, and since the experimental errors on the $f^{\prime}$ two-photon width are so large that it does not matter anyway, the value of the $j$ two-photor width obtained from the non-unitarized model is used. Figure $10.6 a$ compares the DELCO results with $S U(3)$ predictions as a function of the mixing angle. Two separate regions are allowed when solving for the mixing angle, but prior knowledge favors the solution most near to ideal mixing:

$$
\Theta=28^{\circ} \pm 4^{\circ} .
$$

This agrees well with the prediction $\theta \approx 28^{\circ}$ obtained from the mass differences of particles in the tensor noner?7 Figure $10.6 \mathrm{~b}$ shows the comparison between $S U(3)$ predictions and data for the ratio of the $f$ and $A_{2}$ two-photon widths, where the value for the $A_{2}$ two-photon width is taken from Ref. 6 . For a mixing angle of $28^{\circ}$, the $S U(3)$ prediction is outside of the experimental error limits, but only slightly so. Both theoretical and experimental uncertainties are large enough that one must conclude that the data are in agrecment with these simple predictions of the nonrelativistic quark model and $S U(3)$ symmetry. 

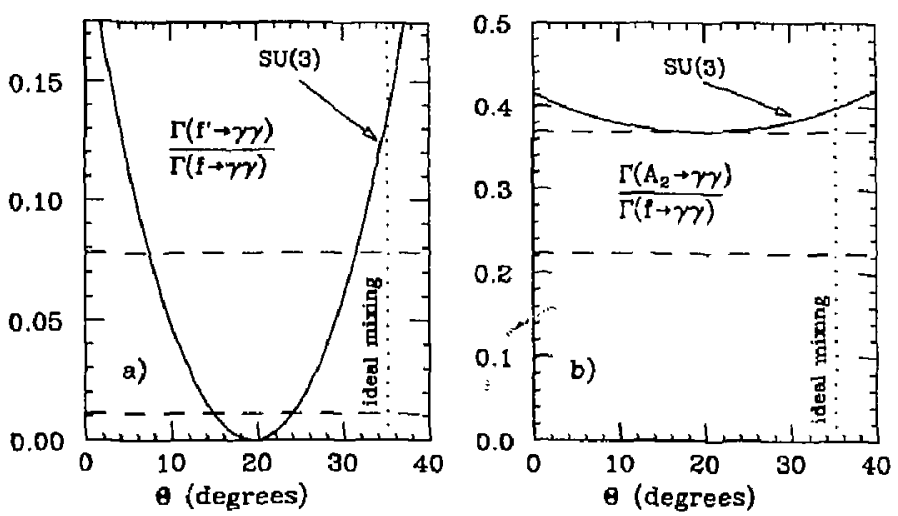

Figure 10.6. $S U(3)$ quark model predictions for the tatios of two-photon widths as functions of the mixing angle. The solid curves represent the predictions of Eqn: 1.10, while the dashed lines give the upper and lower ont-standard-deviation limits on the measured ratios of partial widths.

\subsection{CROSS SECTION FOR $\gamma \gamma \rightarrow p \bar{p}$}

From a sample of 23 events from the process $e^{+} e^{-} \rightarrow e^{+} e^{-} p p$ in the protonpair mass range $2.2<W<2.9 \mathrm{GeV}$, the average cross section within the angular acceptance $-0.6<\cos \theta_{\mathrm{cms}}<0.6$ has been measured to be

$$
\sigma_{\gamma \gamma \rightarrow W \bar{p}}=1.2 \pm 0.5 \mathrm{nb}, \quad(2.2<W<2.9 \mathrm{GeV},|\cos \theta|<0.6) .
$$

The TASSO collaboration has published ${ }^{78}$ results for this channei based on a sample of 72 events, which are enough to see the forms of invariant mass and angular distributions. The angular distribution is flat, while the invaricnt-mass distribution falls from about $4 \mathrm{nb}$ at $W=2 \mathrm{GeV}$ to less than $0.5 \mathrm{nb}$ above $W=3 \mathrm{GeV}$. Averaging the TASSO points over the range measured by DELCO gives $\sigma_{\gamma \gamma \rightarrow p \bar{p}} \approx 1.8 \mathrm{n} 3$ for $-0.6<\cos \theta_{\mathrm{cms}}<0.6$, which is in agreement with the DELCO result.
The Born cross section for $\gamma \gamma \rightarrow p \bar{p}$ integrated over $-0.6<\cos \theta_{\mathrm{cms}}<0.6$ is an average of $24.5 \mathrm{nb}$ over the range $2.2<W<2.9 \mathrm{GeV}$, which is a factor of 20 greater than the DELCO measurement. That is not surprising, since the proton is a poor aforoximation for a point Eirac particle. QCD calculations for this process hav been attempted using methods which are similar to those discussed for meson sairs in Thapter 7 , but considerably more complicated, with many more Feynmar, diagrams and greater uncertainty about the wave functions and normalization. TASSO compares the calculations of Damgaard ${ }^{70}$ with their measurements, and they find that the calculated cross sections are a factor of 3 to 5 low. A nother calcula:ion of the same procias by Farrar et al. ${ }^{80}$ predicts that the cross section is a facior of 20 to 100 lower than the prediction of Ref. 79 and has

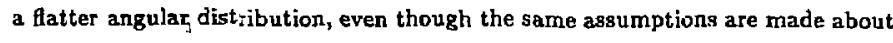
the proton wave function. Farrar et al, estimate that the momentum transfer in the collision should be greater than $5 \mathrm{GeV}$ for such calculations to be valid, especially with an as ; mptotic form for the proton wave function. Since that is at least a factor of two greater than the momentum trassfers observed by DELCO and TASSO, they repeat the calculations with a proton wave function recently propased by Cherny : $:$ and Zhitnitsky ${ }^{B 1}$ and find better agreement with the data. For example, they $t$ edict a cross section of $0.17 \mathrm{nb}$ integrated over the angular range $-0.6<\cos \theta_{\mathrm{cm}}<0.6$ at $W=2.4 \mathrm{GeV}$. 


\section{Appendix A. Integration of the EPA spectrum}

The experimental implications of the EPA spectrum can be studied by making simple integrations with limits determined by only the most significant features of the detector acceptance. For two-prong production, if some additional approximations are made, then the integrations can easily be done analytically, with the nice result that the salient features quickly are exposed in an intuitive manner. Integrations of more complex final states and over more detailed models of the acceptance are done with Monte Carlo techniques.

It is instructive to carry out an integration of the differential cross section for $e^{+} e^{-} \rightarrow e^{+} e^{-} e^{+} e^{-}$over what is roughly the DELCO acceptance, in order to obtain the distribution of the invariant mass of the electron pair which is observed in the central detector. This excrcise gives an understanding of the behavior of the invariant-mass spectrum for production of pairs of fermions and also provides an understanding of the effects of the limited detector acceptance. Electron pairs are of special interest because ultrarelativistic approximations can readily be made and because that is a clannel which is easily observed by DELCO.

A procedure for doing such integrations for general two-prong final states and a number of typical acceptance cuts has been given in detail by $\mathrm{A}$. Courai.?2 Here we give just an outline of the calculation as it is done to obtain the inv.: iant-mass distribution of the electron pairs. The first step is to integrate over the $Q^{2}$ dependence of the photon flux of Eqn. 2.4 from $Q_{\min }^{2}$ up to some value $Q_{\max }^{2}$, which generally is determined by experimental constraints. Defining $\Delta \equiv\left(Q_{\max .}^{2}-Q_{\min .}^{2}\right) / Q_{\min .}^{2}$, one finds

$$
\mathrm{d} n_{i}=\frac{\alpha}{\pi} S\left(x_{i}\right) \frac{\mathrm{d} x_{i}}{x_{i}}
$$

with

$$
\begin{aligned}
S\left(x_{i}\right) & =\left(1-x_{i}+\frac{1}{2} x_{i}^{2}\right) \ln \left(1+\Delta^{2}\right)-\left(1-x_{i}\right)\left[1-1 /\left(1+\Delta^{2}\right)\right\} \\
& \approx 2\left(1-x_{i}+\frac{1}{2} x_{i}^{2}\right) \ln \Delta-\left(1-x_{i}\right) .
\end{aligned}
$$

Using this photon flux along with the cross section for $\gamma \gamma \rightarrow e^{+} e^{-}$, given for the case of relativistic electrons by

$$
\frac{\mathrm{d} \sigma_{\gamma \gamma}}{\mathrm{d} u \mathrm{~d} W}=\frac{2 \pi \alpha^{2}}{W^{2}} \frac{1+u^{2}}{1-u^{2}}, \quad u \equiv \cos \theta_{\mathrm{cms}}
$$

results in the differential cross section

$$
\frac{\mathrm{d}^{3} \sigma}{\mathrm{d} x_{1} \mathrm{~d} x_{2} \mathrm{~d} u}=\frac{\alpha^{4}}{2 \pi E^{2}} \frac{S\left(x_{1}\right) S\left(x_{2}\right)}{x_{1} x_{2}} \frac{2 E}{W} \frac{1+u^{2}}{1-u^{2}} .
$$

In the limit of zero-angle scattering of the beam electrons, the relations $Z^{2} \equiv(W / 2 E)^{2}=x_{1} x_{2}$ and $x_{i}=Z_{e^{ \pm y}}$ may be used, where $y=\tanh ^{-1} \rho$ and $\beta c$ is the velocity of the $\gamma \gamma$ system along the beam axis. Changing variables in Eqn. A.4 gives

$$
\frac{d^{3} \sigma}{\mathrm{d} Z d y \mathrm{~d} u}=\frac{\alpha^{4}}{\pi E^{2}} \frac{1}{Z^{3}} S\left(Z e^{y}\right) S\left(Z e^{-y}\right) \frac{1+u^{2}}{1-u^{2}}
$$

Already it is evident that the behavior of the invariant-massspectrum is dominated by a factor of $1 / W^{3}$. To complete the calculation, both us and $y$ must be integrated over the experimental acceptance.

Let us define the acceptance in such a way that only the most significant limits of DELCO are accounted for. These are the angular acceptance in $\theta$ and the lower momentum limit defined by the detector trigger. Neither quantity is cul of sharply at its limits by the detector itself. In the analysis, however, shiarp cuts are made which are well inside the complicated boundary of the detector hardware. These cuts are made on $\cos \theta_{\text {lab }}$ and $W$, which is convenient for the integration.

If first the rapidity, $y$, and then $\cos \theta$ are integrated over, the integration limits are as follows. The rapidity is limited by both the energy (since $x_{i}<1$ ) and the angular acceptance $\left(|\cos \theta| \leq u_{0}\right)$, so the upper limit on $y$ is the minimum of $\ln (1 / Z)$ and $\left(\tanh ^{-1} u_{0}-\tanh ^{-1} u\right)$. Because of symmetry in $\cos \theta$, it is only 
necessary to integrate over half of the angular range, so the lower limit can be taken to be zero. Then the integration over $u$ can be done from 0 to $u_{0}$.

Finally, the upper limit on the integration over $Q^{2}$ is yet to be specified. If the experimental limit is imposed by anti-tagging of the scattered electrons, then

$$
\Delta_{i}=\frac{E}{m_{e}} \frac{1-x_{i}}{x_{i}}\left(2 \sin \theta_{m} / 2\right),
$$

where $\theta_{m}$ is the minimum tagging angle. Usually, a more important limit is produced by cuts on the transverse momentum, $k_{\perp}$, of the electron pair. When applying either of these limits to the integration, it is passible to assume that all of the contribution near the limits comes from scattering of one beam electron, because the probability for both electrons to scatter at large angles is relatively minute. In that case, $Q^{2} \approx k_{\perp}^{2}$, so a cut on $k_{\perp}$ is directly a cut on the $Q^{2}$ of the virtual photons.

For DELCO, some typical values used are $u_{0}=0.6, W_{\text {min. }}=0.6 \mathrm{GeV}$, and $k_{1} \leq 0.2 W$. Integrating the crose section of Eqn. A.5 over this acceptance yields the masa distribution of Fig. A.la. Because the integration region is rectangular for most values of $W$, there is little deviation of the mass distribution from the simple $1 / W^{3}$ behavior. Included in Fig. A.1 are two additional curves for larger angular acceptances, so one can see the drastic reduction in the cross section due to the limited angular acceptance.

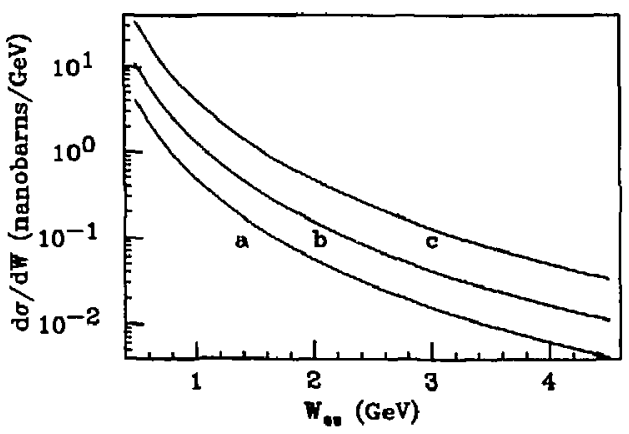

Figure A.1. The invariant-mass differential cross section for $e^{+} e^{-}$pairs from $e^{+} e^{-} \rightarrow e^{+} e^{-} e^{+} e^{-}$. The acceptance for the pair observed in the central detector is defined to be $k_{\perp} \leq 0.2 W$ and $(a)|\cos \theta| \leq 0.60$, (b) $|\cos \theta| \leq 0.80,(c)|\cos \theta| \leq 0.95$ 
Appendix B. Monte Carlo Integration and Event Generation

In Appendix $A$ an integration of the $e^{+} e^{-} \rightarrow e^{+} e^{-} e^{+} e^{-}$cross section was done over a simplified detector acceptance by using the equivalent photon approximation. Any integration involving a more complex region or a more complicated spectrum is best done by Monte Carlo methods. This chapter first provides some general background about the use of Monte Carlo integration and then discusses the opecific calculations used in the data analysis. ${ }^{83}$

\section{B.1 The monte Carlo Method}

The basis for Monte Carlo integration is the integral expression for the meen of a function:

$$
\bar{f}=\frac{1}{v} \int f\left(x_{1} \ldots x_{n}\right) \mathrm{d} V, \quad v \equiv \int \mathrm{d} V .
$$

Since the mean may be approximated by an average over many randomly selected points within the $n$-dimensional volume, then so may the integral. In practice, the integration region often is so complex that the integral $V=\int \mathrm{d} V$ also must be done by Monte Carlo methods. The usual approach is to use a hit-or-miss method. One randomly chooses points from a uniform distribution in an $n$-dimensional box which contains the actual reglon. If a point falls outside the rcgion, then it is rejected. The ratio of the number of points retained to the total number selected times the volume of the box gives the volume of the desired region.

Extending this idea gives a method for generating differential distributions of $f\left(x_{1} \ldots x_{n}\right)$ in any variable $v=v\left(x_{1} \ldots x_{n}\right)$. Any bin in the range of $v$ defines an $n$-dimensional integration region which is contained within the full region. It follows that each time a point is selected which gives a value for $v$ which falls within the ith bin, then that bin should be incremented by the amount $f /\left(\Delta v_{i} \cdot N\right)$, where $\Delta v_{i}$ is the bin width and $N$ is the total number of points selected.

The Monte Carlo procedure gives only an approximation to the integral, so it is important to evaluate the uncertainty in the result. In contrast to most numerical integration methods, this estimate is easy to produce. Its square is given by $\sigma^{2}=V(f) / N$, where $V(f)$ is the variance of the function $f$. Calculating it requires integrating the square of the function, which is done by Monte Cario also. Thus the variance is calculated from the formula

$$
V(f)=\left[\frac{\sum_{j=1}^{N}\left(f\left(x_{1}^{j} \ldots x_{n}^{j}\right)-\bar{f} \cdot{ }^{\prime}\right.}{N}\right]^{\frac{1}{2}} .
$$

If the volume of the region is calculated by the hit-or-miss method, then that introduces further uncertainty. It becomes especially important for the case of small bins in a differential distribution, for which the number of counts in a given bin have a Poisson distribution. Then the additional uncertainty is approximately $\sigma_{i}=\bar{f}_{i} / \sqrt{n_{i}}$, where $\bar{f}_{i}$ is the average of $f$ over the ith bin and $n_{i}$ is the number of bin entries.

It is clear that in all cases the uncertainty decreases like $1 / \sqrt{N}$ as $N$ incresses. It is remarkable that this remains true no matter how many dimensions are included in the integration. Even so, the variance may in fact be so large that as absurdly large $N$ is necessary to bring the error down to a reasonable lovel. That is bound to be a problem if the function to be integrated has one or more large peaks. In such cases, special methods should be used to reduce the variance. One way is to divide the region into a number of pieces, so the integral is a sum of integrations over small regions in which the function does not vary greatly. That alone generally will reduce the variance, but a better reduction is obtained if the number of points chosen in each region is taken to be proportional to the everage function value within that region. An iterative procedure can be used which uses the results of the integration from one iteration to provide the number of points to be calculated in each region for the next iteration.

Such importance sampling often can be done analytically by using explicit knowledge about the behavior of the function to be integrated. The method simply amounts to a change of integration variables. Consider a one-dimensional 
case for aimplicity. The trick is to find a simple function, $g(x)$, which has the same behavior in the peaks as the more complicated $f(x)$. For convenience, the normalizstion $\int_{a}^{b} g(x) \mathrm{d} x=1$ is assumed, where $a$ and $b$ define the boundary for the Monte Carlo integration. For each iteration a uniform random number $\boldsymbol{R}_{j}$ between 0 and 1 is generated, and a new variable $x_{j}$ is calculated from

$$
R_{j}=\int_{a}^{x_{j}} g(y) d_{y,} \quad 0 \leq R_{j} \leq 1 .
$$

After $N$ iterations, th estimate of the integral is given by $(1 / N) \sum_{j} f\left(x_{j}\right) / g\left(x_{j}\right)$. Note that if $g=f$ then the variance is zero. To make use of that fact, however, one must already know the aralytic solution to the integral. It is important to realize that, in the multidimensional case, even if $f=g$ the integral still will have some uncertainty if the region boundaries are so complicated that the hit-or-miss method must be used.

In experimental physics, the most useful sspect of Monte Carlo integration is that it is a simple method for integrating over arbitrarily complex regions. In fact, the region may be taken to be defined by a very detailed simulation of the apparatus, with all sorts of inefficiencies and resolution effects included. Usually histograms are made of the various kinematic variables, just as is done with data. In auch a case the aralysis simplified if the histograms can be accumulated without including weights. That may be done by using the hit-or-miss method. The point in the space $\left\{x_{i}\right\}$ is chosen as usual, using some variance reduction technique if necessary. Let $w$ represent the weight, which simply is $f\left(x_{1} \ldots x_{n}\right)$ if importance sampling is not used. $A$ uniform random number $R$ is selected, and with $w_{\text {max. }}$ being the maximuin weight over the region, the point is rejected if $\boldsymbol{R} \cdot w_{\max }>\boldsymbol{w}$. $A$ lot of calculation time will be wasted if $\boldsymbol{w}$ has any significant peaks, so in practice it often is necessary to divide the region into many subregions and then estimate the maximum weight for each.

An integration made without weights almost always is considerably less efficient than one which uses the weights, so it should be avoided unless there is a good reason for it. The sdvantages of an unweighted event generator often can be more important, however. The simulated events can be treated just as the data are, and the uncertainty in each bin always is simply the square root of the bin contents. Furthermore, it is common for the simulation of the apparatus to require much more computation than the event generation, so there is a danger of wasting a lot of time simulating evento with very small weights if unweighted events are not produced.

\section{B.2 Monte Carlo Integration of the EPA Spectrum}

A Monte Carlo program was written for the DELCO analysis to generate $\gamma \gamma$ states according to the EPA spectrum of equations 2.14 and $2.15{ }^{\text {s4 }}$ It can be used in conjunction with a variety of other programs for generating the final atate from the $\gamma \gamma$ system, and it is interfaced into the DELCO detector simulation software. Here the program is illustrated by considering the specific case of production of lepton pairs.

We assume that the $\gamma \gamma \rightarrow X$ cross section has the typical form

$$
\frac{\mathrm{d} \sigma}{\mathrm{d} u \mathrm{~d} W}=\frac{2 \pi}{W^{2}} f(u, W)
$$

For the EPA spectrum, a change of variables is made from the set $\left\{Q_{1}^{2}, Q_{2}^{2}, x_{1}, x_{2}\right\}$ to the set $\left\{Z, y, t_{1}^{2}, t_{2}^{2}\right\}$, where we have defined

$$
z \equiv x_{1} x_{2}, \quad y \equiv \frac{1}{2} \ln \frac{x_{1}}{x_{2}}, \quad t_{i}^{2} \equiv \frac{Q_{i}^{2}}{Q_{\min .}^{2}}
$$

The limits between which these variables must be integrated should be chosen to be somewhat outside the arceptance of the detector. In particular, $Z$ is only approximately proportional to $W$, so it is a mistake to set the $Z$ limits exectly at the acceptance limits in $W$. For all the produced particles to be within the angular acceptance, it is necessary, though not sufficient, that |tanh 
than $u_{0}$, the upper limit on $\cos \theta$. Since the cross section is dominated by events with $t^{2}$ close to unity, while the experimentsl limits are well out on the tail of the distribution, then the efficiency is not unduly harmed by taking the limits on $t^{2}$ to be large and rejecting events with scattering angles too large for the experiment. $1 \leq t_{i}^{2} \leq E / m_{e}$ is chosen as the appropriate integration rarge.

In the new variables, the cross section is

$$
\mathrm{d} \sigma=\frac{2 \alpha^{2}}{\pi^{2}} S\left(Z e^{y}, t_{1}^{2}\right) S\left(Z e^{-y}, t_{2}^{2}\right) \frac{\mathrm{d} t_{1}^{2}}{t_{1}^{2}} \frac{\mathrm{d} t_{2}^{2}}{t_{2}^{2}} \frac{\mathrm{d} Z}{Z^{3}} \mathrm{~d} y \frac{Z^{2}}{W^{2}} 4 \pi f(u, W) \mathrm{d} u
$$

where $S\left(x, t^{2}\right) \equiv\left[\left(1-x+\frac{1}{2} x^{2}\right)-(1-x) / t^{2}\right]$. The $1 / t_{i}$ and $1 / Z^{3}$ behavior must be removed by importance sampling. To do so, the variables are generated as follows from four uniform random numbers $R_{i}$.

$$
\begin{aligned}
R_{i} & =\frac{1}{\ln \left(E^{2} / m_{e}^{2}\right)} \int_{1}^{t_{i}^{2}} \frac{\mathrm{d} x}{x}, \quad i=1,2 \\
R_{3} & =\left[\frac{1}{2}\left(\frac{1}{Z_{1}^{2}}-\frac{1}{Z_{2}^{2}}\right)\right]^{-1} \int_{Z_{1}}^{Z} \frac{\mathrm{d} x}{x^{3}} \\
y & =\left(2 R_{4}-1\right) \cdot \tanh ^{-1} u_{0} .
\end{aligned}
$$

The first two expressions of Eqn. B.7 are easily inverted to give the formulas

$$
\begin{aligned}
t_{i}^{2} & =\exp \left(2 R_{i} \ln \frac{E}{m_{e}}\right) \\
Z & =\left[\frac{1}{Z_{1}^{2}}-\left(\frac{1}{Z_{1}^{2}}-\frac{1}{Z_{2}^{2}}\right) R_{3}\right]^{-\frac{1}{2}} .
\end{aligned}
$$

Once the variables have been chosen, it is possible to calculate the momenta of the scattered electrons and of the $\gamma \gamma$ state. At that point, the event should be rejected if the scattering angle of either electron is larger than the maximum value allowed experimentally. The weight for the event, aside from additional factors resulting from the cross section for $\gamma \gamma \rightarrow X$, is

$$
\begin{aligned}
w_{\gamma \gamma}=\frac{2 \alpha^{2}}{\pi^{2}} S\left(Z e^{y}, t_{1}^{2}\right) S\left(Z e^{-y}, t_{2}^{2}\right) \\
\times\left[\ln \left(E^{2} / m_{e}^{2}\right)\right]^{2}\left[\frac{1}{2}\left(\frac{1}{Z_{1}^{2}}-\frac{1}{Z_{2}^{2}}\right)\right]\left[2 \tanh ^{-1} t_{0}\right] \frac{Z^{2}}{W^{2}} .
\end{aligned}
$$

The variance of this weight is small, especially compared with the variance of the original integrand, Eqn. B.6. Therefore, the Monte Carlo is efficient, and it is practical to use it for generation of unweighted events. To do so, first the maximum weight is estimated, and then all events with $R_{5} \cdot w_{\gamma \gamma}^{\max }>w_{y \gamma}$ are rejected.

\section{B. 3 INCLUDING THE CROSS SECTION FOR $\gamma \gamma \rightarrow 1^{+}$}

The cross section for production of lepton pairs from two real photons is, to leading order in QED;

$$
\frac{\mathrm{d} \sigma}{\mathrm{d} \Omega}=\frac{\alpha^{2}}{W^{2}}\left[2\left(2-\beta_{l}^{2}\right)-\left(1-\beta_{l}^{2} u^{2}\right)-2 \frac{\left(1-\beta_{l}^{2}\right)^{2}}{1-\beta_{l}^{2} u^{2}}\right] \frac{\beta_{l}}{1-\beta_{l}^{2} u^{2}},
$$

where $\mathrm{d} \Omega=\mathrm{d} u \mathrm{~d} \phi$ is the volume element in the center-of-mass of the $\gamma \gamma$ system. The variable $u=\cos \theta$ is defined with respect to the axis made by the colliding photons in their center-of-raass system, but for untagged events, that is very close to being parallel to the laboratory $z$ axis, along which the electron beams travel. Note that $\beta_{l}=\sqrt{1-\left(m_{l} / k^{0}\right)^{2}}$, where $k^{0}=W / 2$, so the cross section contains a dependence on $W$, which in turn is a function of both $Z$ and $y$. Therefore, the integration does not contain the angular integral as a separate factor. When doing importance sampling in such a multidimensional integral, it usually is only possible to consider one dimension at a time. Since importance sampling is equivalent to a change of variables, if the transformation function used dependo on more than one of the variables, one must be careful to include properly the Jacobian of the transformation. So far, all the changes of variables made to Eqn. 8.6 have involved 
only one variable at a time. Here we make a change of variables from $u$ to $R(u)$ with a dependence on $\beta$, which in turn is a function of both $Z$ and $y$ :

$$
R(u)=\frac{\tanh ^{-1} \beta_{l} u}{\tanh ^{-1} \beta_{l} u_{0}}
$$

In this case, the contribution to the Jacobian simply is

$$
1 / R^{\prime}(u)=\tanh ^{-1} \beta u_{0} \frac{1-\beta_{l}^{2} u^{2}}{\beta_{l}} .
$$

In other words, we can generate $R$ uniformly from 0 to $I$ and calculate $u=\tanh \left(R \tanh ^{-1} \beta u_{0}\right) / \beta$. Then the overall weight for an event is Eqn. B.9 times

$$
4 \pi \alpha^{2}\left[2\left(2-\beta^{2}\right)-\left(1-\beta^{2} u^{2}\right)-2 \frac{\left(1-\beta^{2}\right)^{2}}{1-\beta^{2} u^{2}}\right] \tanh ^{-1} \beta_{l} u_{0}
$$

where an extra factor of $\mathbf{2}$ has been introduced so that the integration can be done on only half of the symmetric range of $-u_{0}$ to $u_{0}$

\section{B.4 MONTE Carlo Fon the Single-Tag Luminosity Function}

For the analysis of tagged events which arc not entirely governed by QED, the single-tag luminosity function of Section 2.4 is used in place of the EPA spectrum. The tet of variahles to generate are for this case $\left\{x_{1}, x_{2}, \theta_{2}\right\}$, where $\theta_{2}$ is the scattering angle of the tagged electron. An equivalent set is $\left\{Z, y, \theta_{2}\right\}$, where $Z$ and $y$ are defined in Eqn. B.5. Again, $Z$ and $y$ are genesated according to the formulas of equations B.7 and B.8, but $\theta_{2}$ is generated according to a cot $\frac{1}{2} \theta_{2}$ diatribution, between the limits $\theta_{\min }$ and $\theta_{\max }$, by the formula

$$
\theta_{2}=2 \sin ^{-1}\left\{\sin \frac{1}{2} \theta_{\min } \exp \left[R \ln \left(\frac{\sin \frac{1}{2} \theta_{\max }}{\sin \frac{1}{2} \theta_{\min }}\right)\right]\right\} \text {. }
$$

where $R$ is a uniform random number between zero and one.
The event weight follows from Eqn. 2.18 and is given by

$$
\begin{aligned}
w & =\frac{\alpha^{2}}{2 \pi^{2}} \ln \left(\frac{\sin \frac{1}{2} \theta_{\max }}{\sin \frac{1}{2} \theta_{\min }}\right)\left(\frac{1}{Z_{1}^{2}}-\frac{1}{Z_{2}^{2}}\right) \tanh ^{-1} u_{0} \\
\times & {\left[\frac{\left(K+2 x_{1}\right)^{2}}{K^{2}}+1\right]\left[\left(\frac{\left(K-2\left(x_{2}+\left(Q_{2} / 2 E\right)^{2}\right)\right]^{2}}{K^{2}}+1\right)\right.} \\
& \left.\times \ln \left(\frac{2 E}{m} \frac{\left(1-x_{1}\right)}{x_{1}} \sin \frac{1}{2} \hat{\theta}_{\min }\right)-2 \frac{1-x_{1}}{x_{1}^{2}}\right] \cdot K \cdot \frac{Z^{4}}{W^{2}} \cdot w_{X},
\end{aligned}
$$

where $K \equiv\left(W^{2}+Q_{2}^{2}\right) / 4 E^{2}, \tilde{\theta}_{\min }$ is the minimum tagging angle for the apparatus, and $w_{X} / W^{2}$ is the weight for the process $\gamma \gamma \rightarrow X$. Although it is by no means obvious at a glance, for the range of $\theta$ available for tagging in DELCO this weight has a reasonsbly small variance, so it can be used for genteration of unweighted events without any serious inefficiency, as long as $w_{X}$ also is reasonably well optimized.

\section{B.5 Calculatiúns Without EPA}

If it is necessary to investigate a region of $Q^{2}$ in which EPA is not valid, then more complex calculations must be porformed. Monte Carlo programs have been written to handle some specific cases. ${ }^{22,32}$ When the Feynman diagrams are complicated and large in number, it is prohibitively difficult to sum the amplitudes and square them analytically. Therefore, the programs calculate the complex amplitudes numericaily, add them together, and square them. Much care muat be exercised to avoid numei ical instabilities.

The program which we use is the one of Ref. 22 for the process $e^{+} e^{-} \rightarrow$ $e^{+} e^{-1+1-}$. There are contributions to this process in addition to the two double-bremsstrahlung (multiperipheral) diagrams, but they give no significant contribution to the cross section in the experimental situation where only the $l^{+} r^{-}$ are observed in the detector ${ }^{32}$ Six diagrams are included in Ref. 22, whereas for the four-electron final state there actually are a total of thirty six. The six which 

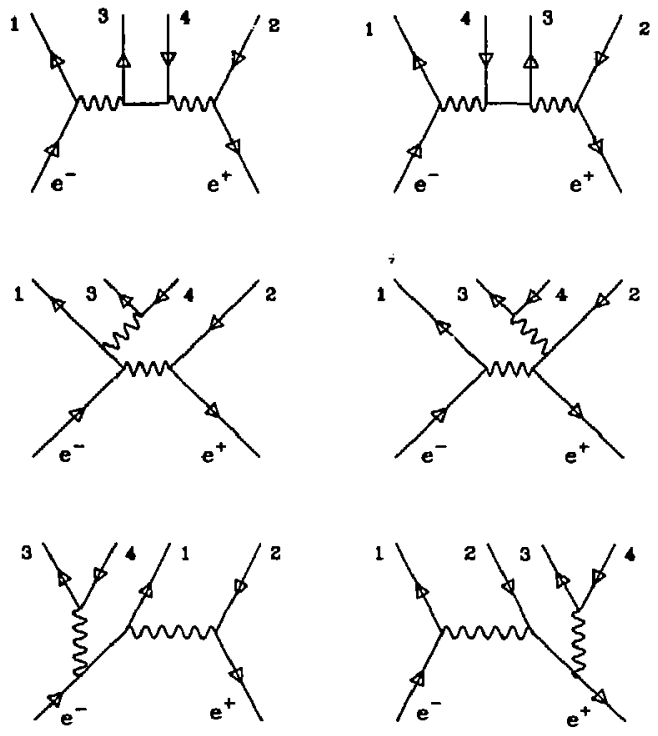

Figure B.1. Six diagrams for the process $e^{+} e^{-} \rightarrow e^{+} e^{-1+} l^{-}$. Thest are the diagrams included in the calculation of Ref. 22.

are included are shown in Fig. B.1. The diagrams in which the beam electrons annihilate are entirely neglected, and for the case of four final-state electrons, interference between the fermions originating in the beam and those originating from the $\gamma \gamma$ interaction is neglected. A general purpose adaptive Monte Carlo routine called VEGAS ${ }^{86}$ is used to do the integration. VEGAS works by the method of importance sampling, but rather than using continuous functions to approximate the integrand, which would require prior knowledge of the integrand, it uses step functions, which are adjusted iteratively to adapt to the integrand in use.
The procedure is to divide each axis of the $n$-dimensional space into $N$ intervals. Then the probability of choosing a point from any given interval is defined to be a constant. Initially the intervals along an axis are of equal length. During the first iteration a specified number of function evaluations are made. They give estimates for the mean values of the function in each interval, which are used to change the grid spacing for the next iteration. The intervals are made more narrow where the function value is relatively large, 80 in the next iteration more points will be concentrated there than where the function is small.

All function evaluations in all iterations are used in the determination of the integral and its uncertainty. Individual iterations also produce a value for the integral and an associated error estimate. The $x^{2}$ of all the values should not greatly exceed the number of iterations, or else one must suspect that the procedure has becorne unstable. Such an instability could be due to making not enough function evaluations in each iteration. That number and the number of iterations must be chosen by the user. A good choice will result in a reasonable $\chi^{2}$ per iteration and a standard deviation per iteration which decreases at first but levels of beiore the last iteration is made.

The program also ean be used to generate unweighted events.7 To do so, VEGAS is run through several iterations just for the purpose of finding the optimum grid spacing. At this time, the rough limits of the detector acceptance are included by defining the integrand to be zero outside of it. Therefore, after the initial iteration, points will not be chosen which lie outside of the acceptance. After the grid spacing is specified, another program considers the behavior of the integrand and estimates its maximum for each interval. After that, points are chosen according to the optimized grid and kept or rejected according the ratio of the function value to the maximum of the interval. This procedure provides for efficient generation of events within the detector acceptance even with such a complicated integrand.

It is of interest at this point to compare the Vermaseren program with the 


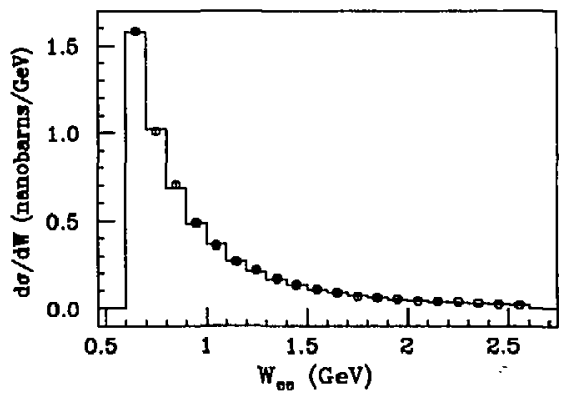

Figure B.2. Two calculations of the $e^{+} e^{-}$invariant-mass distribution The line histogram is from the EPA Monte Carlo, and the pointg are from the Vermaseren Monte Carlo.

EPA Monte Carlo described in the previous section. Figure B.2 and Fig. B.3 show tor the electron pair observed within the DELCO acceptance the invariant mass and angular distributions. The acceptance has been simplified and is defined by only the cuts $0.6 \mathrm{GeV} \leq W \leq 2.6 \mathrm{GeV},-0.6 \leq \cos \theta \leq 0.6$, and $k_{\perp} \leq \min (0.2 W, 0.3 \mathrm{GeV})$. Within such an acceptance, EPA calculates well even the distribution of total transverse momentum $\left(k_{\perp}\right)$, as shown in Fig. B.3. It is clear that as long as a strict cut is made on $k_{1}$, one can expect the EPA to be valid for all of the untagged analysis. Any resulting theoretical errors will be much smaller than the statistical uncertainty of the data.

The full leading-order calculation, including all 36 diagrams, has been done by Berends, Daverveldt, and Kleiss. ${ }^{32}$ They also use variance reduction techniques to handle the sharp peaks that the cross section makes in various regions of the sevendimensional space, but they do so explicitly, rather than rely upon an adaptive Monte Carlo integration routine. Different subsets of diagrams have differing peaking properties. For example, we already have seen from the EPA calculation of the first two diagrams in Fig. 4.1 (the multiperipheral diagrams) that they are
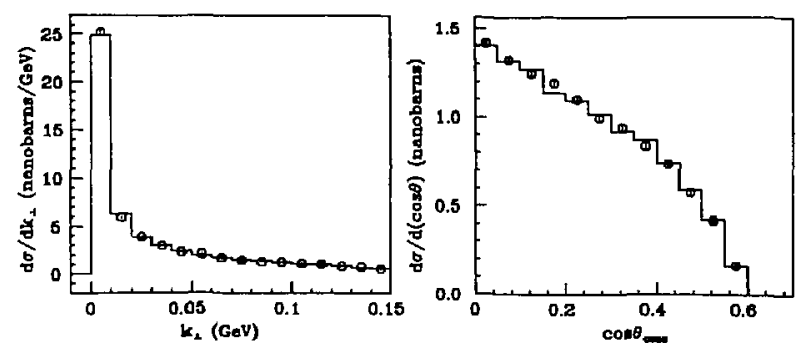

Figure B.3. Two calculations of the $e^{+} e^{-} k_{\perp}$ and $\theta_{\text {cms }}$ distributions. The line histogram is from the EPA Monte Carlo, and the points are from the Vermaseren Monte Carlo.

characterized by sharp peaks at low invariant mass and low $Q^{2} \cdot \dagger$ Other subsets have peaks in different regions of phase space.

The procedure used works by separating the diagrams in to six subsets, where all diagrams in a particular subset give amplitudes which have a sharp peak, arising when the $q^{2}$ of one or more of the propagators becomes small, in the same region of phase space of the external particles. In each Monte Carlo iteration, one of the subsets is chosen with a probability proportional to its approximate contribution to the total cross section. An event is generated according to just the cross section for the chosen subset, so it is possible to choose the kinematic variables in a way which will cancel the peaks in that subset. The resulting sample of events is corrected by calculating for each the full cross section according to the entire set of diagrams and applying a rejection 2 gorithm.

One result of their calculations is that for the experimental situation where only two of the electrons pass through the central detector, the two multiperipheral diagrams completely dominate the cross section. Therefore, for the DELCO

$\uparrow$ For the four-electron final state, EPA includes only twa of the permutations of the outgoing electron lines, thus ignoring interference between the scattered beam electrons and those from the ry system. 
analysis it is a safe approximation to use the Vermaseren Monte Carlo, or even to use the EPA Monte Carlo when untagged events are analyzed.

\section{Appendix C. Unfolding Methods for Experimental Distrihutions}

The procedure used in this thesis for unfolding kinematic distributions from the measured histograms is due to V. Blobel. In Ref. 88 he gives an excellent account of the justification and need for the method, a derivation of the equations, and an example calculation. Here we provide only a brief summary of how the calculation is done. The principle difference from Blobel's example is that, whereas his data is one dimensional, here we always must consider a multidimensional problem.

Even here, though, only one dimension is considered at a time. The problem with the other dimensions is only that they must be modeled properly in order that the efficiency corrections for the variable under consideration be correct. As a concrete example, let us consider unfolding the two-particle invariant mass distribution. In this case, for example, it is clear that the result will be correct only if the angular distributions are correct in the Monte Carlo which is uged to calculate the efficiency. Let $W$ represent the invariant mass, and let $W_{a} \leq W \leq W_{b}$ be the range in which we are interested. $f(W)$ represents the function to be determined-the result of the unfolding procedure.

The data is in the form of a histogram with $n$ bins oi equal width, where the value chosen for $n$ depends on the number of events available and also on the experimental resolution of $W$. The extent to which $f(W)$ can be resolved is limited by the statistical precision of the data, so it must be represented by a finite number of parameters $a_{1}, a_{2} \ldots a_{m}$, according to some expansion

$$
f(x)=\sum_{i=1}^{m} a_{i} p_{i}(x) .
$$

The number chosen for $m$ must be less than $n$, but otherwise it is not critical, unless it is much too small, because the regularization procedure to be diseussed will automatically adjust the number of independent coefficients in the final result to be consistent with the statistical precision of the data. 


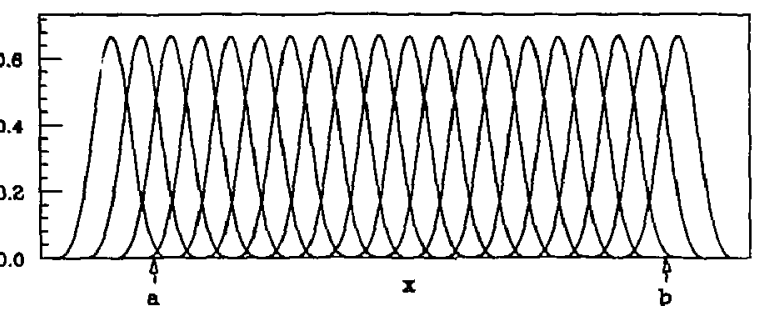

Figure C.1. A sequence of 20 cubic B-splines. They have the property that at any point $x$ between $a$ and $b$, the sum of all of the B-splines is unity.

The functions used for the $p_{i}(x)$ are cubic B-splines with equidistant knots. The range from $W_{a}$ to $W_{b}$ is divided into $m-3$ intervals of equal length $d=\left(W_{b}-W_{a}\right) /(m-3)$, and each of the B-splines is non-zero over a range of 1d. Let $t_{i}$ represent the position of the ith knot, so $t_{i}=W_{a}, t_{m+1}=W_{b}$, and in general, $t_{i}=W_{a}+(i-4) / d$. Then the cubic $B$-splines are given by

$$
b_{i}(x)=\left\{\begin{array}{lll}
\frac{1}{6} z^{3} & z=\left(x-t_{i}\right) / d & t_{i} \leq x<t_{i+1} \\
\frac{1}{6}[1+3(1+z(1-z)) z] & z=\left(x-t_{i+1}\right) / d & t_{i+1} \leq x<t_{i+2} \\
\frac{1}{6}[1+3\{1+z(1-z))(1-z)] & z=\left(x-t_{i+2}\right) / d & t_{i+2} \leq x<t_{i+3} \\
\frac{1}{6}[1-z]^{3} & z=\left(x-t_{i+3}\right) / d & t_{i+3} \leq x<t_{i+4} \\
0 & \text { otherwise }
\end{array}\right.
$$

Figure C.1 shows an example of 20 cubic B-splincs spanning the interval in $x$ between $a$ and $b$.

Now let us represent the response function of the detector as $A(y, x)$, so the invariant-mass distribution which is observed is given by

$$
g(W)=\int_{W_{a}}^{W_{b}} A(W, x) f(x) \mathrm{d} x=\sum_{i=1}^{m} a_{i} A_{i}(W)
$$

where the $A_{i}(W)$ are given by

$$
A_{i}(W)=\int_{W_{a}}^{W_{b}} A(W, x) p_{i}(x) \mathrm{d} x .
$$

The function $g(W)$ is what actually is measured, but only as a histogram with a limited number of events, and therefore some statistical errors. The $A_{i}(W)$ also are produced as histograms from Monte Carlo events. The Monte Carlo events are generated according to some distribution $f_{0}(W)$, and all detector effects are simulated. After analyzing the Monte Carlo events just as is do: a for the data, $m$ histograms of the measured invariant mass $\widetilde{W}$ are accumulated with weights given by $N \cdot p_{i}(W)$, where $W$ is the generated invariant mass. $N$ is an overall normalization given by the ratio of the integrated luminosity in data to the integrated luminosity generated by the Montc Carlo.

Since this really is a multidimensional problem, in order for the efficiencies to be calculated correctly and efficiently by Monte Carlo, it is best if $f_{0}$ is a reasonable approximation to $f$, and it if is necessary that all other kinematic variables ase distributed in a close approximation to reality. That always may be checked by comparing the observed distributions with the Monte Carlo distributions after detector simulation. Thus the bin contents of the $m$ histograms form an $n$-by-m matrix of elements $A_{i j}$, and Eqn. C.3 becomes

$$
g_{i}=\sum_{j=1}^{m} A_{i j} a_{j}
$$

Actually, this is completely true only if $f_{0}=1$; otherwise the resulting $A_{i j}$ are such that the $f(W)$ produced by the unfolding procedure mut be multiplied by $f_{0}(W)$ to give the desired result.

Given $\hat{g}_{i}$, the measured bin contents, Eqn. C.5 may be solved by a maximum likelihood method. The observed bin contents follow a Poisson distribution, for which the probability of observing the number $\hat{g}_{i}$ of events in the ith bin is $P\left(\hat{g}_{i} \mid g_{i}\right)$, 
where

$$
P(r \mid \mu)=e^{-\mu} \frac{\mu^{r}}{r !} \quad r \in\{0,1 \ldots \infty\}
$$

The $g_{i}$ are the average or expected values of the bin contents, which we take to be given by Eqn. C.5; hence $P\left(\hat{g}_{i} \mid g_{i}\right)$ is a function of the parameters $a_{j}$. The likelihood function is formed from the product of the $P\left(\hat{g}_{i} \mid g_{i}\right)$ for all $n$ bins, and its negative logarithm is proportional to

$$
S(a)=\sum_{i=1}^{n} g_{i}-\sum_{i=1}^{n} \hat{g}_{i} \ln g_{i} .
$$

The desired solution is the set of $a_{j}$ which minimizes $S(a)$. However, one finds that such a solution is dominated by large oscillations due to amplification of the statistical errors inherent in the data.

To damp out the oscillations, a regularization term $\frac{1}{2} r \cdot r(a)$ is added to the $\log$ likelihood function, where $r(a)$ is a measure of the curvature of the solution $f(W)$ :

$$
r(a)=\int_{W_{a}}^{W_{b}}\left[f^{\prime \prime}(x)\right]^{2} \mathrm{~d} x=\sum_{i, j=1}^{m} a_{i} C_{i j} a_{j} .
$$

$C$ is a constant, symmetric, positive-semidefinite matrix and is easily calculated for the cubic B-splines. The regularization parameter $\tau$ is adjusted to an appropriate value as explained below.

The solution is calculated as follows. First define the derivative matrices

$$
h_{i}=-\frac{\partial S}{\partial a_{i}} \quad H_{i j}=\frac{\partial^{2} S}{\partial a_{i} \partial a_{j}} .
$$

These must be calculated assuming some initial guesses $\tilde{a}_{i}$ of the parameters $a_{i}$. What is done is first to calrulate the solution assuming Gaussian distributions for the bin contents, in which case the log likclihord function is quadratic in a and no initial guess is newded. The Gaussian result then is used as a starting point for the firat iteration of solving the problem with Poisson statistics. In practice, few iterations are necessary-for high statistics the Gaussian approximation is itself good enough. Once the derivative matrices have been calculated, then the matrix $U_{1}$ is found which diagonalizes $H$, and another matrix $U_{2}$ is found which diagonalizes the matrix

$$
C_{1} \equiv D^{-1 / 2} U_{1}^{T} C U_{1} D^{-1 / 2}, \text { where } D=U_{1}^{T} H U_{1} \text {. }
$$

First consider the unregularized solution $(r=0)$, which we denote by accenting with a bar. It may be written as

$$
\bar{f}(W)=\sum_{i=1}^{m} \mathbf{a}_{j}^{\prime} p_{j}^{\prime}(W)
$$

where the $p_{j}^{\prime}(x)$ are orthonormal polynomials given by

$$
\begin{aligned}
p_{i}^{\prime}(x) & =\sum_{j=1}^{n}\left(U_{1} D^{-1 / 2} U_{2}\right)_{j i} P_{j}(x), \\
\bar{a}^{\prime} & =U_{2}^{T} D^{-1 / 2} U_{1}^{T}(H \tilde{a}+h) .
\end{aligned}
$$

In this orthogonal space, denoted by accenting with a prime, the covariance matrix of the coefficients $\bar{a}_{i}^{l}$ is simply the unit matrix. Therefore, any coefficient which satisfies $\left(\bar{a}_{i}^{\prime}\right)^{2} \leq 3.84$ is statistically compatible with zero at the $95 \%$ confidence level.

The contributions to the curvature of the individual orthonormal polynomials are given by $S_{i i}$, where $S=U_{2}^{T} C_{1} U_{2}$. The coefficients $\overline{\mathrm{a}}_{i}^{\prime}$ should be arranged in order of increasing $S_{i i}$. Let $m_{0}$ be the smallest integer such that for $i>m_{0}$, all $\bar{a}_{i}^{\prime}$ are compatible with zero. The coefficients of the regularized solution are given by

$$
\hat{a}_{i}^{\prime}=\frac{1}{1+r S_{i i}} \bar{a}_{i}^{\prime}
$$

so if we choose $\tau$ such that

$$
m_{0}=\sum_{i=1}^{m} \frac{1}{1+r S_{i i}},
$$


then the coefficients are cut off moothly at the point where they become statistically compatible with zero. The covariance matrix of the regulated solution is

$$
V\left(\hat{a}^{\prime}\right)_{i j}=\frac{\delta_{i j}}{\left(1+r \cdot S_{i i}\right)^{2}}
$$

and both it and the coefficients $\hat{\mathbf{a}}^{\prime}$ may be transformed back to the original space by use of the matrix $U_{1} D^{1 / 2} U_{2}$.

The final task is to convert the coefficients to a set of $m_{0}$ data points by integrating the solution $\hat{f}(W)$ over small contiguous regions of $W$ and dividing each integral by the length of the particular region. The regions are chosen an being between the extrema of the polynomial $p_{m_{0}+1}^{\prime}(W)$. Thus they are located sbout the $m_{0}$ zeroes of that polynomial, which has the effect of suppressing the contribution to the solution from the term $\hat{a}_{m 0+1}^{\prime} p_{m o+1}^{\prime}(W)$ and also tends to give wider bins in regions of less statistical precision and reduce the correlations between data points. Since the integrals over the function $\hat{f}(W)$ are linear functions of the coefficients $\hat{a}_{i}$, then it is straightforward to calculate the covariance matrix for the data points as a linear tranaformation of the covariance matrix for the coefficients.

As mantioned above, if it is necessary, as is usually the ease, that the Monte Carlo in put distribution $f_{0}(W)$ not be uniform, then the unfolded data points must be multiplied by $f_{0}(W)$ to obtain the final result. In fact, for a multidimensional problem, $f_{0}$ is itself the result of an integration over several dimensions and is therefore usually not known analytically. What is done is to use the Monte Carlo generator itself to do the integration, giving a histogram representation of $f_{0}$. That histogram then is interpolated by cubic splines and integrated over the same regions in $W$ as was the solution $\hat{f}$, which gives a set of Monte Carlo points to be directly multiplied by the $m_{0}$ points of the unfolded solution.

Finally, one has a measurement of the physical distribution of interest without any diatortion due detector effects which are unique to the particular experiment.
The procedure is complicated because it is necessary to smooth ostillatory behavior in manner which is compatible with the statistical precision and therefore unbiased. But the complications are worthwhile, because the result is meaningful even to one who is not familiar with the details of the experimental apparatus and methods. Often the result does not contain as many points as the original histograms, and the error bars may be larger. But that also is an advantage, because the points and error bars represent the true limitations of the apparatus. Resolutions effects inevitably decrease the statistical accuracy of an experiment, so the result should properly reflect those limitations. 


\section{REFERENCES}

1. S.J. Brodsky and G.P. Lepage, Large-Angle Two-Photon Exclusive Channels in Quantum Chromodynamics, Phys. Rev. D 24 (1981) 24.

2. J.F. Gunion, D. Millers, and K. Sparks, $Q^{2}$ Dependence of Exclusive Meson Production in Two-Photon Reactions, UC Davis preprint (July 1985) 45pp.

3. J.R. Snith et al. (Mark II collaboration), Pion Pair Production from $\gamma \gamma$ Collisjons at PEP, Phys. Rev. D 30 (1984) 851.

4. J. Boyer et al. (Mark II collaboration), Charged Meson Pair Production in $\gamma \gamma$ Intersetions, SLAC-PUB-3780 and LBL-20280 (Sept. 1985) submitted to Phys, Rev. Lett.

5. W.G.J. Langeveld, Pion and Kaon Pair-Production in Photon-Photon Collisions, Ph.D. Thesis, Utrecht (1985).

6. Particle Data Group, Review of Particle Properties, Rev. Mod. Phys. 56 (April 1984).

7. M. Althoff et al. (TASSO collaboration), Production of $K \bar{K}$-Pairs in Photon-Photon Collisions and the Excitation of the Tensor Meson $I^{\prime}(1515)$, Phys. Lett. $121 B$ (1983) 216.

8. H. Kolanoski, Two-Photon Physics at $e^{+} e^{-}$Storage Rings, Springer-Verlag, Berlin (1984).

9. C. Edwords et al. (Crystal Ball collaboration), Production of $\pi^{0} \pi^{0}$ and $\pi^{0} \eta$ in Photon-Photon Collisions, Phys. Lett. 110B (1982) 82.

10. P. Grassberger and R. Kōgerler, Sum Rules for Elastic $\gamma \gamma \boldsymbol{\gamma}$ Scattering and Meson Decays into Two Photons, Nucl. Phys. B 106 (1976) 451.

11. M.S. Chanowitz, "Resonances in Photon-Photon Scattering," Proceedings of the VIth International Workshop on Photon-Photon Collisions, ed. R.L. Lander, World Scientific, Singapore (1985).

11. S.L. Adler, Axial-Vector Vertex in Spinor Electrodynamics, Phys. Rev. 177 (1969) 2426 .
J.S. Bell and R. Jackiw, A PCAC Puzzle: $\pi^{0} \rightarrow \gamma \gamma$ in the $\sigma$-Model, Nuovo Cimento 60A (1969) 47.

13. Ch. Berger, "Two Photan Physics in the PLUTo Detector," Proceedings of the International Workshop on $\gamma \gamma$ Collisions, Amiens, ed. G. Cochard and P. Kesgler, Lecture Notes in Physics Vol. 134. Springer-Verlag, Berlin (1980).

14. J.D. Bjorken and S.D. Drell, Relativistic Quantum Mechanics, McGrawHill, New York (1964).

15. V.M. Budnev et al., The Two-Photon Particle Production Process, Physics Reports $15 C$ (1975) 241.

16. V.M. Budnev et al, op. eit, equation (5.12).

17. V.M. Budnev et al., op. cit., page 242.

18. V.M. Budnev et al., op. cit., page 246.

19. H. Kolanoski, op. cit., Section 2.4.2.

20. J.H. Field, Luminosity Functions for Two-Photon Processes in $e^{+} e^{-}$ Collisions, Nucl. Phys. B 168 (1980) 477.

Ch. Berger and J.H. Field, Luminosity Function for Two-Photon Processes in the Single Tagging Mode, Nucl. Phys. B 187 (1981) 585.

21. G. Bonneau, M Gourdin, and F. Martin, Inelastic Lepton (Anti)-Lepton Scattering and the Two-Photon Exchange Approximation, Nucl. Phys. B 54 (1973) 573.

22. J. Smith, J.A.M. Vermaseren, and G. Grammer, Two-Photon Production Processes at High Energy, Phys. Rev. D 15 (1977) 3280.

23. J.J. Sakurai, Vector Meson Dominance and High-Energy Electron-Positron inelastic scattering, Phys. Rev. Lett. 22 (1969) 981.

24. J.J. Sakurai and D. Schildknecht, Generalized Vector Dominance and Inelastic Electron-Proton Scattering, Phys. Lett. $40 B$ (1972) 121. I.F. Ginzburg and V.G. Serbo, Some Comments on the Total $\gamma \gamma \rightarrow$ Hadron Section at High Energy, Phys. Lett. 109B (1982) 231. 
25. G.B. Bowden, et al., A Sandwich Structure Beam Pipe for Storage Rings, Nucl. Instr. \& Meth. 228 (1984) 207.

26. V.L. Highland, Some Practical Remarks on Multiple Scattoring, Nucl. Inst. \& Meth. 120 (1975) 497, and Erratum, Nucl. Inst. \& Meth. 161 (1979) 171.

27. H. Hinterberger and R. Winston, Efficient Light Coupler for Threshold Cerenkov Counters, Rev. Sci. Instr. 37 (1966) 1094.

28. D.E. Koop, Prompt Electron Production in Electron-Pasitron Annihilation at $29 \mathrm{GeV}, \mathrm{Ph} . \mathrm{D}$. thesis, Galifornia Institute of Technology (1984).

29. R.L. Ford and W.R. Nelson, The ECS Code System: Computer Programs for the Monte Carlo Simulation of Electromagnetic Showers (Version 3), SLAC-0210 (1978) 277pp. We use Vergion 4, which is to be documented in SLAC-0265. However, it is very similar to Version 3.

30. K.C. Chandler and T.W. Armstrong, Operating Instructions for the FighEnergy Nucleon-Meson Transport Code HETC, ORNL-4744, Oak Ridge Nat. Lab. (1972) 33pp.

T.W. Armstrong et al., Nucl. Scj. Eng. 48 (1972) 82.

T A. Gabriel, J.D. Amburgey, and B.L. Bishop, "CALOR:" A Monte Carlo Program Package For the Design and Analysis of Calorimeter Systems, ORNL/TM-5619, Oak Ridge Nat. Lab. (April 1977) 29pp.

31. H. Yamamoto, A Study of Charged $D^{*}$ Mesons Produced in $e^{+} e^{-}$ Annihilation at $E_{\mathrm{cm}}=29 \mathrm{GeV}$, Ph.D. thesig, California Institute of Technology (1985).

32. F.A. Berends, P.H. Daverveldt, and R. Kleiss, Complete Lowest Order Calculation for Four-Lepton Final States in $\mathrm{e}^{+} \mathrm{e}^{-}$collisions, Nucl. Phys. B 253 (1985) 441.

P.H. Daverveldt, Monte Carlo Simulation of Two-Photon Processes, Ph.D. thesis, Leiden (1985).
33. R. Kleiss, Monte Carlo Simulation of Radiative Processes in ElectronPositron Scattering, Ph.D. thesis, Leiden (1982).

34. G.B. Mills, Charged Kaon Production in Tau Lepton Decays, Ph.D. thesis, Californja Institute of Technology (1985).

H.D. Dahmen, L. Schulke, G. Zech, Contributions of Woa: Interactions to the Process $e^{+} e^{-} \rightarrow \nu_{\tau} X^{+} \nu_{\tau} X^{-}$, Zeit. Phys. C5 (1980) 71.

35. W.L. van Neerven and J.A.M. Vermaseren, The Role of the Five-Point Function in Radiative Corrections to Two-Photon Physics, Phya. Lett. $142 B$ (1984) 80.

36. F.A. Berends, P.H. Daverveldt, and R. Kleiss, Radiative Corrections to the

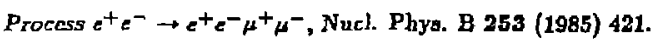

37. G.R. Mills, Internal DELCO memorandum dated Nov. 16, 1993.

38. T. Sjöstrand, The Lund Monte Carlo for $\mathrm{e}^{+} \mathrm{e}^{-}$Jet Physics, Comput. Phys. Commun. 28 (1983) 229.

T. Sjöstrand, The Lund Monte Carlo for Jet Fragmentation, Comput. Phys. Commun. 27 (1982) 243.

39. Ch. Berger as al. (PLUTO collaboration), Messurement of Exclusive $\eta^{\prime}$ Production in $\gamma \gamma$ Reactions, Phys. Lett. 142B (1984) 125.

40. G.P. Lepage and S.J. Brodsky, Exclusive Processes in Perturbative Quantum Chromodynamics, Phys. Rev. D 22 (1980) 2157.

41. See, for example, A. Roussarie et af. (Mark II collaboration), Pion-Pajr Production in Photon-Photon Collisions at SPEAR, Phys. Lett. 105B (1981) 304 .

42. J. Werle, Relativislic Theory of Reactions, North-Holland, Amsterdam (1966).

43. J. Werle, op. eit., equations 28.1 and 28.2 .

44. J. Werle, op. cit., equation 29.21 .

45. J. Werle, op. eit., equations 29.31 and 29.38 . 
16. C.N. Yang, Selection Rules for the Dematerialization of a Particle into Two Photons, Phys. Rev, 77 (1950) 242.

17. Morgan and G Shaw, Pion-Pion Scattering Below $850 \mathrm{MeV}$. A Unique Solution, Phys. Rev. D 2 (1970) 520.

18. G. Hohler et al., Handbook of Pion-Nucleon Scattering, Karlsruhe, Germany, Fachinformationszentrum Energie, Phys., Math (1979) 440 pp.

49. R.J. Eden, P.V. Landshof, D.I. Olive, and J.C. Polkinghorne, The Analytic S Matrix, Cambridge Univ. Press (1966) 287 pp.

50. H.D.I. Abarbanel and M.L. Goldberger, Low Energy Theorems, Dispersion Relations, and Superconvergent Sum Rules for Compton Scattering, Phys. Rev. 165 (1968) 1594.

51. D.H. Lyth, Theoretical Inve $\sin i$ on of the Process $\gamma \gamma \rightarrow \pi \pi$ at Low Energies, Nucl. Phys. B 3c . 471) 19.3.

52. A.D. Martin and T.D. Sperr: . - -ementary Particle Theory, North Holland, Amsterdam (1970) Chapter 8.

53. D.H. Lyth, The Shape of the $f$ in $\gamma \gamma \rightarrow \pi \pi$, Lancaster preprint (Sept. 1984).

54. C.D. Froggatt and J.L. Petersen, Phase-Shift Analysis of $\pi^{+} \pi^{-}$Scattering Between 1.1 and $1.8 \mathrm{GeV}$ Based on Fixed Momentum Transfer Analyticity, Nucl. Phys. B 91 (1975) 454.

55. K.M. Watson, Some General Relations between the Photoproduction and Scattering of $\pi$ Mesons, Phys. Rev. 95 (1954) 228

50. D.H. Lyth, Theoretical Description of the Process $\gamma \gamma \rightarrow \pi \pi$ Up To the $f$ Region, J. Phys. G 10 (1984) 39.

57. See V.M. Budnev et al., op. eit., page 217, for a review of the currentalgebra theorems.

58. L. Luknazuk, A Generalization of the Watson Theorem, Phys. Lett. A7B (1973) 51.
59. G. Mennessier, Meson Pair Production in $\gamma \gamma$ Scattering and Implications for Scalar Mesons, 2. Phys. C 16 (1983) 241.

60. We thank Dr. A. Courau for this suggestion and for making the required modifications to the Mennessier code.

61. H.J. Behrend et al. (CELLO collaboration), Production of the $f_{0}(1270)$ Meson in Photon-Photon Collisions, Z. Phys, C 23 (1984) 223.

62. A.N. Vall, A.E. Kaloshin, and V.V. Serebryakov, The Process $\gamma \boldsymbol{\gamma} \rightarrow$ $f(1270) \rightarrow \pi \pi$ and Low-Energy Parameters, Novosibirsk preprint 144(3) (1985).

63. F. James and M. Roos, MINUIT-A System for Function Minimization and Analysis of the Parameter Errors and Correlations, Comp. Phys. Comm. 10 (1975) 343.

64. A. Courau, et al. (DELco Collaboration), Pion-Pair Produetion from $\gamma \gamma$ Interactions at PEP and the Radiative Width of the $f^{0}$ Meson, Phys. Lett. $147 B$ (1984) 227.

65. J.M. Blatt and V.F. Weisskopf, Theoretical Nuclear Physics, John Wiley and Sons, New York (1952) pp. 359-365 and pp. 386-389.

j6. H. Krasemann, J.A.M. Vermaseren, The $2 \gamma$ reaction $e^{+} e^{-} \rightarrow e^{+} e^{-} \pi^{+} \pi^{-}$ in the Resonance Region, Nucl. Phys. B 184 (1981) 269.

67. U. Fano, Ann. Rev. Nucl. Sci. 13 (1963) 1.

68. R. Talman, On the Statistics of Particle Identification Using Ionization, Nucl. Instr, and Meth. 159 (1979) 189.

69. H.J. Lipkin, Simple Branching Ratios and Sum Rules in Poripheral Reactions, Nucl. Phys. B 7 (1968) 321.

70. Ch. Berger et al. (PLUTO collaboration), Lepton and Hadron Pair Production in Two-Photon Reactions, Phys. Lett. 94B (1980) 254.

B. Adeva et al. (Mark-J collaboration), Experimental Tests of Higher Order Quantum Electrodynamics at Small Distances, Phys. Rev. Lett. 48 (1982) 721. 
H.J. Behrend et al. (CELLO collaboration), Lepton Pair Production in Deep Inelustic ty Seattering, Phys. Lett. 126B (1883) 384.

71. A. Courau et al. (DM1 collaboration), Measurement of Lepton Pair Production in $\gamma \gamma$ Processes from $e^{+} e^{+}$Collisions at DCI, Phys. Lett. 84B (1979) 145.

A. Courau et al. (DM1 collaboration), Measurement of Lepton and Pion Pair Production in Photon-Photon Collisions at DCI, Phys. Lett. 96B (1980) 402.

72. Ch. Berger et al. (PLUTO collaboration), Lepton and Hadron Pair Production in Two-Photon Reactions, Phys. Lett. 04B (1980) 320.

73. R. Brandelik et al. (TASSO collaboration), Two-Photon Excitation of the Tensor Meson $f^{\circ}$ (1270), Z. Phys. C 10 (1981) 117.

74. Ch. Berger et al. (PLUTO collaboration), Pion Pair Production in PhotonPhoton Interactions, Z. Phys. C 28 (1984) 199.

75. P. Jenni et al. (Mark II collaboration), Resonance Production by TwoPhoton Interactions at SPEAR, Phys. Rev. D 27 (1983) 1031.

76. N.P. Samios, M. Goldberg, and B.T. Meadows, Hadrons and $S U(3)$ : A Critical Review, Rev. Mod. Phys. 16 (1974) 49.

R. Ammar et al., $f^{*}$ Production in $K^{-}$p Interactions at $5.5 \mathrm{GeV} / \mathrm{c}$, Phys. Rev. Lett. 19 (1967) 1071

V.E. Barnes et al., Properties of the $f^{*}$ Meson Phys. Rev, Lett. 19 (1967) 964.

77. See, for example, the discussion of the nonrelativistic quark model in Ref. 6, page S44.

78. M. Althoff et al. (TASSO collaboration), Differential Cross Sections for $\gamma \gamma \rightarrow p \bar{p}$ in the CM Energy Range Prom 2.0 to $3.1 \mathrm{GeV}$, Phys. Lett. 130B (1983) 449.

R. Brandelik et al. (TASSO collaboration), Exclusive Proton-Antiproton Production in Two-Photon Collisjons, Phys. Lett. 108B (1982) 67.
79. P.H. Damgaard, Exclusive Processes in QCD: Two-Photon Physics Involving Baryons, Nuel. Phys. B 211 (1983) 435.

80. G.R. Farar, E. Maina, and F. Neri, QCD Predictions for $\gamma \boldsymbol{\gamma}$ Annihilation to Baryons, Rutgera University print RU-85-08 (April 1985) 35pp.

81. V.L. Chernyak and I.R. Zhitnitsky, Nucleon Wave Functions and Nucleon Form Factors in QCD, Nucl. Phys. B 246 (1984) 52.

82. A. Courau, Analytic Distributions of Various Parameters in ee $\rightarrow e e x^{+} x^{-}$ Under Standard Experimental Conditions, Phys. Rev. D 29 (1984) 24.

83. A useful introduction to practical Monte Carlo methods is F. James, Monte Carlo Theory and Practice, Reports on Progress in Physics 43 (1980) 1145.

84. The program is based on a similar program written by $A$. Courau, which he has docurnented in the report A Fast Monte Carlo Generator for ee $\rightarrow$ ceX Untagged Experiments, SLAC-PUB-3363 (June 1984).

85. J.M. Jauch and F. Rohrlich, The Theory of Photons and Electrons, Addison-Wesley, Reading Massachusetts (1955) equation 13-37.

86. G.P. Lepage, A New Algorithm for Adaptive Multidimensional Integration, J. Comput. Phys. 27 (1978) 192.

87. See the manual for the use of AXOLIB, the library of the Vermaseren routines (unpublished).

88. V. Blobel, Unfolding Methods in High-Energy Physics Experiments, DESY 84-118 and CERN Comp. School (1984) 88. 